\title{
Membrane Dish Analysis: A Summary of Structural and Optical Analysis Capabilities
}

C.R. Steele, C.D. Balch, G.J. Jorgensen,

T. Wendelin, and A. Lewandowski

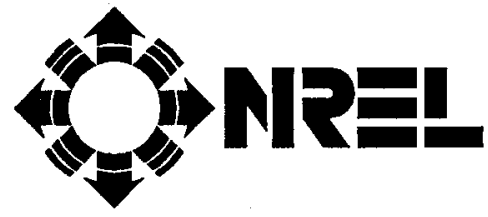

National Renewable Energy Laboratory 1617 Cole Boulevard

Golden, Colorado 80401-3393

A division of Midwest Research Institute operated for the U.S. Department of Energy under contract No. DE-AC02-83CH10093 


\section{Membrane Dish Analysis: A Summary of Structural and Optical Analysis Capabilities}

C.R. Steele, C.D. Balch, G.J. Jorgensen, T. Wendelin, and A. Lewandowski

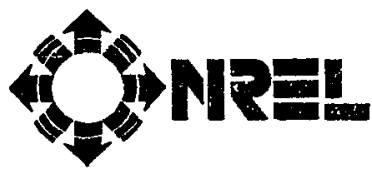

National Renewable Energy Laboratory (formerly the Solar Energy Research Institute) 1617 Cole Boulevard Golden, Colorado 80401-3393

A Division of Midwest Research Institute Operated for the U.S. Department of Energy under Contract No. DE-AC02-83CH10093

Prepared under Task No. SE112031

November 1991 


\section{On September 16, 1991, the Solar Energy Research Institute was designated a national laboratory, and its name was changed to the National Renewable Energy Laboratory.}

\section{NOTICE}

This report was prepared as an account of work sponsored by an agency of the United States government. Neither the United States government nor any agency thereof, nor any of their employees, makes any warranty, express or implied, or assumes any legal liability or responsibility for the accuracy, completeness, or usefulness of any information, apparatus, product, or process disclosed, or represents that its use would not infringe privately owned rights. Reference herein to any specific commercial product, process, or service by trade name, trademark, manufacturer, or otherwise does not necessarily constitute or imply its endorsement, recommendation, or favoring by the United States government or any agency thereof. The views and opinions of authors expressed herein do not necessarily state or reflect those of the United States government or any agency thereof.

Printed in the United States of America

Available from:

National Technical Information Service

U.S. Department of Commerce

5285 Port Royal Road

Springfield, VA 22161

Price: Microfiche A01

Printed Copy $\mathrm{A} 10$

Codes are used for pricing all publications. The code is determined by the number of pages in the publication. Information pertaining to the pricing codes can be found in the current issue of the following publications which are generally available in most libraries: Energy Research Abstracts (ERA); Government Reports Announcements and Index (GRA and I); Scientific and Technical Abstract Reports (STAR); and publication NTIS-PR-360 available from NTIS at the above address. 


\section{Preface}

The research and development described in this document was conducted within the U.S. Department of Energy's (DOE) Solar Thermal Technology Program. The goal of this program is to advance the engineering and scientific understanding of solar thermal technology and to establish the technology base from which private industry can develop solar thermal power production options for introduction into the competitive energy market.

Solar thermal technology concentrates the solar flux using tracking mirrors or lenses onto a receiver where the solar energy is absorbed as heat and converted into electricity or incorporated into products as process heat. The two primary solar thermal technologies, central receivers systems and distributed receivers, employ various point and line-focus optics to concentrate sunlight. Current central receiver systems use fields of heliostats (two-axes tracking mirrors) to focus the sun's radiant energy onto a single, tower-mounted receiver. Point focus concentrators up to 17 meters in diameter track the sun in two axes and use parabolic dish mirrors of Fresnel lenses to focus radiant energy onto a receiver. Troughs and bowls are line-focus tracking reflectors that concentrate sunlight onto receiver tubes along their focal lines. Concentrating collector modules can be used alone or in a multimodule system. The concentrated radiant energy absorbed by the solar thermal receiver is transported to the conversion process by a circulating working fluid. Receiver temperatures range from $100^{\circ} \mathrm{C}$ in low-temperature troughs to more than $1500^{\circ} \mathrm{C}$ in dish and central receiver systems.

The Solar Thermal Technology Program is directing efforts to advance and improve each system concept through solar thermal materials, components, and subsystems research and development and by testing and evaluation. These efforts are carried out with the technical direction of DOE and its network of field laboratories that work with private industry. Together they have established a comprehensive, goal-directed program to improve performance and provide technically proven options for eventual incorporation into the nation's energy supply.

To successfully contribute to an adequate energy supply at reasonable cost, solar thermal energy must be economically competitive with a variety of other energy sources. The Solar Thermal Technology Program has developed components and system-level performance targets as quantitative program goals. These targets are used in planning research and development activities, measuring progress, assessing alternative technology options, and developing optimal components. These targets will be pursued vigorously to ensure a successful program. 
Approved for

SOLAR ENERGY RESEARCH INSTITUTE

Zan a. millem

T. Williams, Manager

Solar Thermal Program

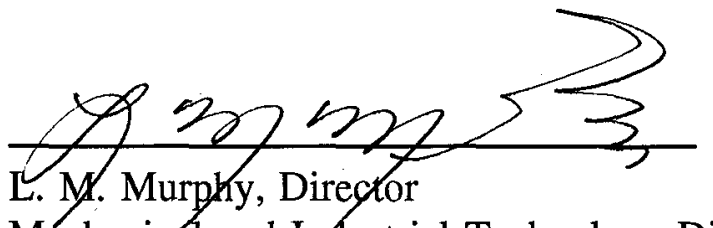

Mechanical and Industrial Technology Division 


\section{Executive Summary}

Research at SERI within the Department of Energy's Solar Thermal Technology Program has focused on the development of membrane dish concentrators for space and terrestrial power applications. As potentially lightweight, inexpensive, high-performance structures, they are excellent candidates for space-deployable energy sources as well as cost-effective terrestrial energy concepts.

A thorough engineering research treatment of these types of structures consists primarily of two parts: (1) structural mechanics of the membrane and ring support and (2) analysis and characterization of the concentrator optical performance. It is important to understand the effects of the membrane's structure and support system on the optical performance of the concentrator. This requires an interface between appropriate structural and optical models. Until recently, such models and the required interface have not existed.

This report documents research that has been conducted at SERI in this area. It is a compilation of several papers describing structural models of membrane dish structures and optical models used to predict dish concentrator optical and thermal performance. The structural models were developed under SERI subcontract by Dr. Steele and Dr. Balch of Stanford University. The optical model was developed in-house by SERI staff. In addition, the interface between the models is described. It allows easy and thorough characterization of membrane dish systems from the mechanics to the resulting optical performance.

The models described herein have been and continue to be extremely useful to SERI, industry, and universities involved with the modeling and analysis of lightweight membrane concentrators for solar thermal applications. 


\section{Contents}

Section

$\underline{\text { Page }}$

1. Structural Mechanics of the Membrane and Ring Support

1.1 Edge Effects in a Shell of Revolution under Pressure Load . . . . . . . . . . 1.1-1

1.2 Non-Axisymmetric Deformations of Thin Paraboloidal Shells with Initial Prestress . . . . . . . . . . . . . . . . . . . . . . .

1.3 Procedures and Software for Dish Membrane Structural Analysis . . . . . . . 1.3-1

2. Non-Axisymmetric Optical Model of Solar Dish Concentrators $\ldots . \ldots \ldots$ 2-1

Appendix A - Asymptotic Solutions for Edge Effects in Thin Shells $\ldots . \ldots \ldots$ A-1

Appendix B - Non-Axisymmetric Deformations of Paraboloidal Shells with

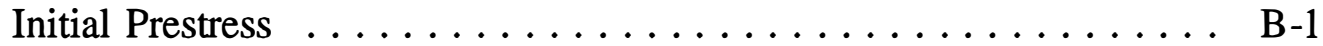

Appendix C - Structural and Structural/Optical Analysis of Dish Membranes .... C-1 


\section{Figures and Tables}

\section{Section 1.1}

Figure 1. Coordinate system for axisymmetric shell . . . . . . . . . . 1.1-2

Figure 2. Force components and displacement components for shell under pressure ......................... 1.1-3

Figure 3. Paraboloidal shell subjected to uniform pressure load $\ldots \ldots \ldots \ldots$ 1.1-12

Figure 4. Solution for meridional bending stress in shell under pressure . . . . 1.1-15

Figure 5. Solution for meridional bending stress near edge of shell under

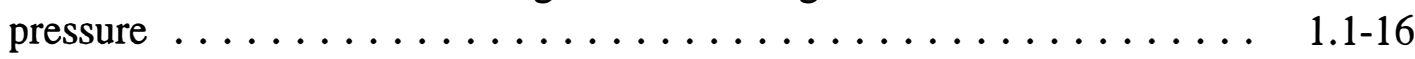

Figure 6. Rotation for shell under pressure load . . . . . . . . . . . 1.1-17

Figure 7. Solution for meridional rotation near edge of shell under pressure . . . 1.1-17

Figure 8. Stress resultants for shell under pressure . . . . . . . . . . . 1.1-18

Figure 9. Horizontal and vertical displacement for shell under pressure . . . . . 1.1-20

Table 1. Specifications for paraboloid, from Murphy $(1987) \ldots \ldots \ldots \ldots$ 1.1-13

\section{Section 1.2}

Figure 1. Shell element, coordinate system, and reference basic vectors . . . . . 1.2-3

Figure 2. Stress and moment resultants acting on shell element $\ldots \ldots \ldots \ldots$ 1.2-3

Figure 3. Meridian of shell of revolution and meridional arclength

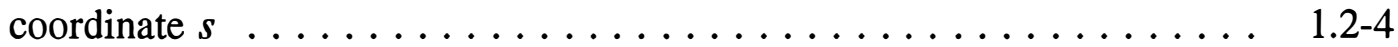

Figure 4. Kinematic degrees of freedom along an edge $\ldots \ldots \ldots \ldots \ldots \ldots$ 1.2-4

Figure 5. Decay distance $\delta$ as a function of $n$ for two edge bending solutions . . 1.2-12

Figure 6. Radial and vertical displacement for shell subjected to $\cos 2 \Theta$ radial edge displacement . . . . . . . . . . . . . . . . . 1.2-19

Figure 7. Rotations for shell subjected to $\cos 2 \Theta$ radial edge displacement $\ldots . . \quad 1.2-20$

Figure 8. Stress resultants for shell subjected to $\cos 2 \Theta$ radial edge

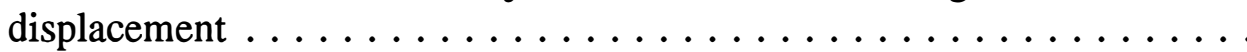

Figure 9. Radial and vertical displacement for shell subjected to $\cos n \Theta$ radial edge displacements . . . . . . . . . . . . . . . .

Figure 10. Radial and vertical displacement for shell subjected to $\cos 2 \Theta$

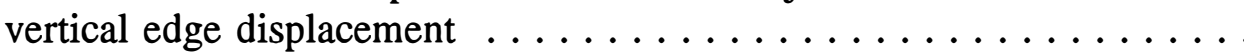

Figure 11. Stress resultants for shell subjected to $\cos 2 \Theta$ vertical edge displacement . . . . . . . . . . . . . . . . . . .

Figure 12. Radial and vertical displacement for shell subjected to $\cos n \Theta$ vertical

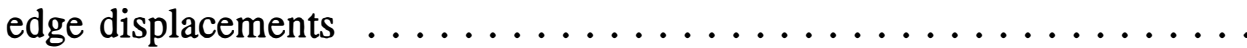

Figure 13. Stress resultants for clamped shell subjected to asymmetric load

$\begin{aligned} & p_{n}=p_{w} \sin \phi \cos \Theta \ldots \ldots \ldots \ldots \ldots \ldots \ldots \ldots \ldots \ldots \ldots \ldots \ldots \ldots \ldots\end{aligned}$

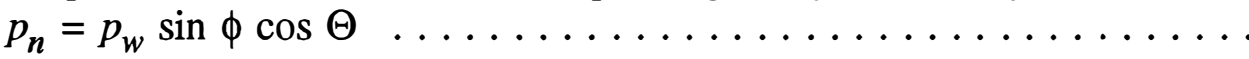




\section{Figures and Tables (Continued)}

Section 1.2 (continued)

$\underline{\text { Page }}$

Figure 15. Meridional rotation for shell with clamped edge subjected to asymmetric

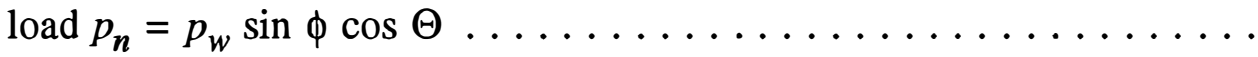

Table 1. Specifications for paraboloid dish $\ldots \ldots \ldots \ldots \ldots \ldots \ldots \ldots \ldots$ 1.2-18

Table C- . Sample input file for the DISH program . . . . . . . . . . . 1.2-33

Table C-2. Sample output file DISH.DAT, from the DISH program $\ldots \ldots \ldots \ldots$ 1.2-35

Table C-3. Sample output file DISH.OUT, from the DISH program $\ldots \ldots \ldots \ldots$. $1.2-36$

\section{Section 1.3}

Figure 1. Flowchart of data paths for structural and structural/optical analyses . . 1.3-22

Figure 2. Sign conventions for forces, displacements, moments, and rotations at edge of dish . ........................ 1.3-23

Figure 3. Data path flowchart for enforcing finite-element nodal ring displacements in the dish structural analysis . . . . . . . . . . . 1.3-24

Figure 4. Comparison of SUPERSAP nodal displacements Uz with the nine-term Fourier series representation . . . . . . . . . . . . . . . . . .

Figure 5. Meridional and tangential stress resultants Ns and Nst due to ring displacements . . . . . . . . . . . . . . . . . . . . 1.3-26

Figure 6. Distribution of rays at target plane for head-on wind load $\ldots \ldots \ldots$ 1.3-27

Figure 7. Distribution of rays at target plane for wind load $\ldots \ldots \ldots \ldots \ldots$ 1.3-28

Figure 8. Distribution of rays at target plane for $1 \mathrm{~cm} \mathrm{n}=2$ radial displacement . . . . . . . . . . . . . . . . . . . . . . . 1.3-29

Figure 9. Distribution of rays at target plane for ASDF4E edge displacements . . 1.3-30

Figure 10. Distribution of rays at target plane for ASDF4E edge displacements . . 1.3-31

Table 1. Sample input file DISH.IN for the DISH program . . . . . . . . . . 1.3-13

Table 2. Sample input file DFCOMP.IN for the post-processor DFCOMP . . . . 1.3 13

Table 3. Sample output file DFCOMP.OUT for the post-processor DFCOMP . . . . 1.3-14

Table 4. Output from the postprocessor DINFO . . . . . . . . . . . . 1.3-15

Table 5. Nodal displacements and rotations from a SUPERSAP analysis . . . . 1.3-15

Table 6. Input file FSCOEF.IN to the Fourier analysis program FSCOEF . . . . . 1.3-16

Table 7. Output file FSCOEF.OUT from the Fourier analysis program FSCOEF . . 1.3-17

Table 8. Input file DISH.IN with prescribed edge displacements from FSCOEF.OUT . . . . . . . . . . . . . . . . . . . . . . . . 1.3-18

Table 9. Input file FCOMPN.IN to the program FCOMPN . . . . . . . . . 1.3-19

Table 10. Output file FCOMPN.OUT of nodal forces and moments from FCOMPN $1.3-19$

Table 11. Input file DISH.IN for membrane solution only . . . . . . . . . . 1.3-20

Table 12. Change in nodal forces and moments relative to the membrane solution,

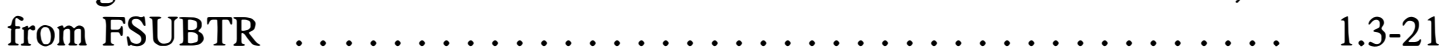




\section{Figures and Tables (Concluded)}

Section 2 Page

Figure 1. Logic flow and interfaces of OPTDSH . . . . . . . . . 2-2

Figure 2. Example GENDAT program which generates .DAT finite element-like grid points on a polar basis . ................ 2-3

Figure 3. Example GENDAT program which generates .DAT finite element-like grid points on a cartesian basis ............... 2-4

Figure 4. Example of .DAT input file which uses Zernike monomials . . . . . 2-5

Figure 5. Example of .DAT input file for analytic surface $\ldots \ldots \ldots \ldots \ldots .2-8$

Figure 6. Example of .DAT input file for modeling welded seams $\ldots \ldots \ldots \ldots 2-8$

Figure 7. Sample OUTPUT.SAV file . . . . . . . . . . . . . . 2-9

Figure 8. Sample THERML.DAT file $\ldots \ldots \ldots \ldots \ldots \ldots \ldots \ldots \ldots \ldots . \ldots \ldots$

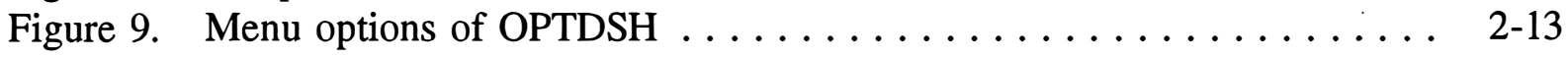

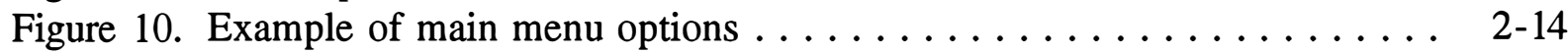

Figure 11. Example of configuration option menu ............. 2-14

Figure 12. Example of system parameter specification options $\ldots \ldots \ldots \ldots \ldots$ 2-14

Figure 13. Example of error parameter specification options $\ldots \ldots \ldots \ldots .2-15$

Figure 14. Example of thermal analysis parameter specification options $\ldots \ldots \ldots$ 2-15

Figure 15. Example of analysis session using OPTDSH $\ldots \ldots \ldots \ldots \ldots \ldots .2-16$

Figure 16. Example of graphical display options menu $\ldots \ldots \ldots \ldots \ldots \ldots .2-17$

Figure 17. Sample frequency histogram . ............... 2-18

Figure 18. Distribution of randomly generated rays on the dish surface; zonal binning is out to $0.2 \mathrm{~m}$ in the target plane $\ldots \ldots \ldots \ldots \ldots \ldots . \ldots .2-19$

Figure 19. Distribution of 2000 randomly generated rays in the target plane . . . 2-20

Figure 20. Thermal efficiency as function of aperture radius $\ldots \ldots \ldots \ldots \ldots .2-22$

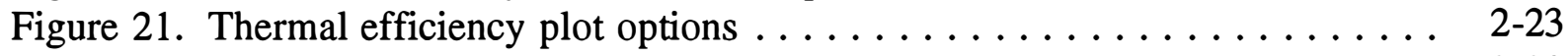

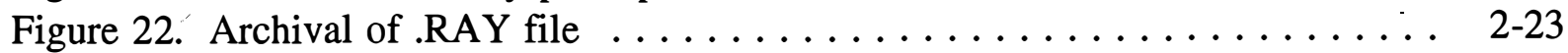

Figure 23. Example of DISH.DAT input file used with SHAPE option . . . . . 2-24

Table 2-1. Description of parameters contained in input files to OPTDSH $\ldots \ldots$ 2-5 
Section 1

\section{Structural Mechanics of the Membrane and Ring Support}




\title{
Edge Effects in a Shell of Revolution under Pressure Load
}

\author{
submitted to the Solar Energy Research Institute by \\ C. R. Steele* and C. D. Balch§ \\ Division of Applied Mechanics \\ Stanford University \\ Stanford, California 94305
}

SHELLTECH report 87-1, August 1987

* Professor of Mechanical Engineering

$\S$ Research Associate 


\begin{abstract}
The governing fourth-order equations are presented for the shell of revolution under axisymmetric load, and an asymptotic solution for edge bending is obtained. The asymptotic solution is based on the assumption that the thickness of the shell is very small relative to the the shell's radii of curvature. In order to incorporate the effect of high pressurization on edge bending, the geometric nonlinearity associated with the change in meridional slope is included in the governing equations.

The total solution, which is the sum of the membrane (particular) and edge bending (complementary) solutions, is given for the case of uniform pressure load, and the steps required in order to evaluate the solution are explicitly described. As an example, the paraboloidal shell under uniform pressure load is discussed, and the results are compared with a previous ANSYS finite element analysis by Murphy (1987).
\end{abstract}




\section{$\S 1$ Introduction}

In many thin shell structures, loads are carried chiefly by membrane stresses. Near the edges of a shell, however, boundary constraints may give rise to bending stresses which are rapidly varying functions of the spatial coordinates. For the case of the thin, axisymmetric shell, this type of local edge effect can be described by an asymptotic solution, which exploits the fact that the shell thickness $t$ is very small compared to other dimensions of the shell. This report presents an asymptotic solution for edge bending effects in a thin, axisymmetric shell, and focuses on the case of uniform pressure loading.

The shell geometry and coordinate system are shown in Fig. 1. Figure 1 shows the meridian curve of the shell, which is defined by a function $y(r)$. The angle $\phi$, given by

$$
\phi=\tan ^{-1}\left(\frac{d y}{d r}\right)
$$

is the angle between the $y$ axis and the normal to the meridian.

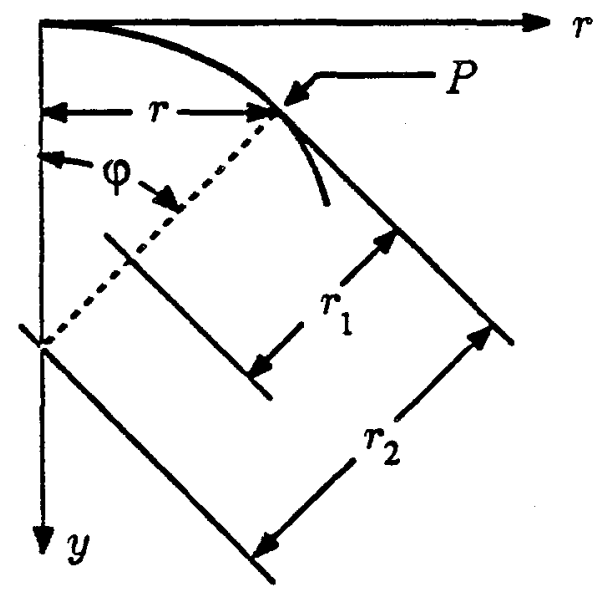

Figure 1. Coordinate system for an axisymmetric shell. The distances $r_{1}$ and $r_{2}$ are the radii of curvature in the $\phi$ (meridional) and $\theta$ (circumferential) directions at the point $P$. 
Also shown in Fig. 1 are the radii of curvature of the shell. The meridional radius of curvature is denoted by $r_{1}$; thus an element $d s$ of arc length along the meridian is given by

$$
d s=\sqrt{d r^{2}+d y^{2}}=r_{1} d \phi
$$

The radius of curvature $r_{2}$ in the circumferential direction is given by

$$
r_{2}=\frac{r}{\sin \phi}
$$

The force quantities to be employed in the analysis are shown in Fig. 2a. The quantity $N_{\phi}$ is the meridional stress resultant (force per unit length), and $Q$ is the transverse shear resultant. For thin shells, it can usually be assumed that $Q \ll N_{\phi}$. The stress resultant vector may also be expressed in terms of the horizontal and vertical components $H$ and $V$ (see Fig. 2a). The meridional bending moment is denoted by $M_{\phi}$.

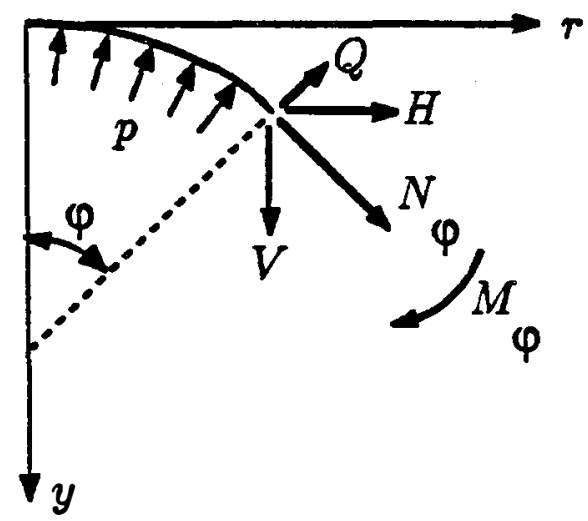

(a)

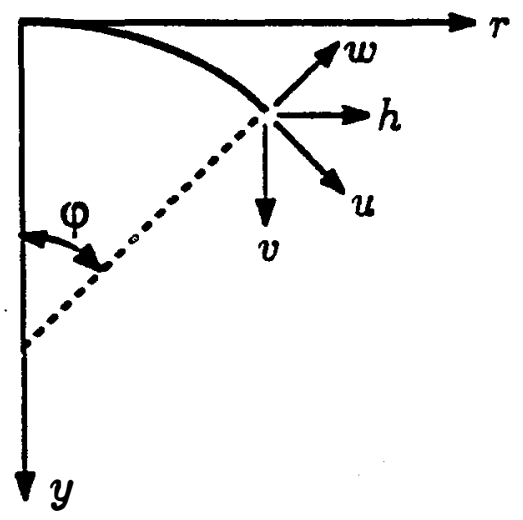

(b)

Figure 2. (a) Force components and uniform pressure load $p$; and (b) displacement components for the axisymmetric shell.

Figure $2 \mathrm{~b}$ shows the shell displacements, which can be defined in terms of the tangential and normal components $(u, w)$ or the horizontal and vertical components $(h, v)$.

\section{§2 Governing Equations}

The governing equations for a shell of revolution under axisymmetric 
load can be written in "state vector" form as follows:

$$
\begin{array}{r}
\left.-\frac{d}{d s}\left\{\begin{array}{c}
M_{\phi} \\
H \\
\chi \\
h
\end{array}\right\}+\left[\begin{array}{cccc}
-\frac{(1-\nu) \cos \phi}{r} & \sin \phi & \frac{E t^{3} \cos ^{2} \phi}{12 r^{2}} & 0 \\
0 & -\frac{(1-\nu) \cos \phi}{r} & 0 & \frac{E t}{r^{2}} \\
\frac{1}{E t c^{2}} & 0 & -\frac{\nu \cos \phi}{r} & 0 \\
0 & \frac{\left(1-\nu^{2}\right) \cos ^{2} \phi}{E t} & -\sin \phi & -\frac{\nu \cos \phi}{r}
\end{array}\right] \begin{array}{c}
M_{\phi} \\
H \\
\chi \\
h
\end{array}\right\} \\
=\left\{\begin{array}{c}
V \cos \phi \\
p_{H}-\frac{\nu \sin \phi}{r} V \\
-\frac{\left(1-\nu^{2}\right) \sin \phi \cos \phi}{E t} V
\end{array}\right\}
\end{array}
$$

in which the coordinate $s$ is the arc length along the meridian, $E$ is the elastic modulus, $\nu$ is Poisson's ratio, $t$ is the shell thickness, and $c$ is the reduced thickness, defined by

$$
c=t /\left[12\left(1-\nu^{2}\right)\right]^{\frac{1}{2}}
$$

The quantities $r, \phi, M_{\phi}, H, V$, and $h$ in Eq. (4) are defined in Fig. 2. The load term $p_{H}$ is the horizontal ( $r$ direction) component of the load, and the displacement quantity $\chi$ is the rotation of the meridian, given by

$$
\chi=-\frac{d w}{d s}+\frac{u}{r_{1}}
$$

The four coupled first-order differential equations (4) follow from the equilibrium, kinematic, and constitutive relations for the shell of revolution. The derivation of Eq. (4) is discussed in Steele and Skogh (1970).

\section{$\S 3$ Geometric Nonlinearity due to Pressurization}

For a thin shell under internal or external pressure, it is possible for the magnitude of the membrane stresses to be of the same order as the magnitude of the local buckling stress. Under these circumstances, it is necessary to consider the geometric nonlinearity associated with the change in meridional slope. (This is analogous to a stability or prestress analysis in classical beam theory, in which a geometrically nonlinear second-order term must be added to the fourth-order ODE.)

The relationship between the stress resultants $\left(N_{\phi}, Q\right)$ and $(H, V)$ (see Fig. 2a) therefore becomes

$$
\begin{aligned}
N_{\phi} & =H \cos (\phi+\chi)+V \sin (\phi+\chi) \\
& =(H \cos \phi+V \sin \phi)-(H \sin \phi-V \cos \phi) \chi+O\left(\chi^{2}\right) \\
Q & =H \sin (\phi+\chi)-V \cos (\phi+\chi) \\
& =(H \sin \phi-V \cos \phi)+(H \cos \phi+V \sin \phi) \chi+O\left(\chi^{2}\right)
\end{aligned}
$$


where the slope of the deformed meridian is $\phi+\chi$. In most problems where the meridional rotation $\chi$ is moderate, the meridional stress resultant vector remains parallel to the undeformed meridian curve; hence the membrane result

$$
H=V \cot \phi
$$

remains accurate. The second term of Eq. $(7 a)$ and the first term of Eq. (7b) are thus negligible, along with the terms of $O\left(\chi^{2}\right)$.

Incorporating the nonlinear corrections ( 7 ) into the equation of moment equilibrium results in a small formal modification of the governing equations. Let $A$ represent the $4 \times 4$ coefficient matrix of Eq. (4). The only significant change due to the geometric nonlinearity is in the $(1,3)$ component of $\mathbf{A}$ :

$$
A_{13}=\frac{E t^{3} \cos ^{2} \phi}{12 r^{2}}+\frac{2 E t c}{r_{2}} \rho
$$

where

$$
\rho=\frac{r_{2}(H \cos \phi+V \sin \phi)}{2 E t c}=\frac{p r_{2}^{2}}{4 E t c}
$$

is a dimensionless "pressurization parameter." For $|\rho| \ll 1$, the effects of the geometric nonlinearity are negligible, but for $|\rho|=O(1)$, the effects are significant. The value $\rho=-1$ corresponds to the classical buckling load for the shell.

\section{$\S 4$ Asymptotic Solutions}

In order to obtain an asymptotic solution to Eq. (4) for the shell of revolution under axisymmetric load; it is first necessary to write Eq. (4) in dimensionless form, and to identify a suitable "large" parameter in the problem. When carried out properly, these steps render Eq. (4) in the following form:

$$
-\frac{1}{\lambda} \frac{d y}{d x}+\left(A_{0}+\frac{1}{\lambda} A_{1}+\frac{1}{\lambda^{2}} A_{2}\right) y=\lambda\left(b_{0}+\frac{1}{\lambda} b_{1}+\frac{1}{\lambda^{2}} b_{2}\right)
$$

in which $x$ is the dimensionless arc length coordinate, defined by

$$
x=s / r_{2 e}
$$

and $\lambda$ is a dimensionless large parameter, defined by

$$
\lambda=\left(r_{2 e} / c_{e}\right)^{\frac{1}{2}} \gg 1
$$

The quantities $r_{2 e}$ and $c_{e}$ are the circumferential radius of curvature $r_{2}$ and the reduced thickness $c$ evaluated at the edge of the shell. 
In Eq. (11), the vector $\mathbf{y}$ of dimensionless unknowns is defined by

$$
\mathbf{y}=\left\{\begin{array}{c}
\frac{M_{\phi}}{(E t c)_{e}} \\
\frac{\lambda \sin \phi_{e} H}{(E t)_{e}} \\
\chi / \lambda \\
h / r_{e}
\end{array}\right\}
$$

where the subscript $e$ denotes that a quantity is evaluated at the edge of the shell. The $4 \times 4$ matrices $\mathbf{A}_{(0,1,2)}$ and the $4 \times 1$ right-hand side vectors $\mathbf{b}_{(0,1,2)}$ in Eq. (11) are also dimensionless; their components are given explicitly in Steele and Skogh (1970).

The solution y to Eq. (11) can be separated into its particular and complementary parts:

$$
y=y^{(p)}+y^{(c)}
$$

where $\mathbf{y}^{(p)}$ denotes a particular solution to Eq. (11), and $\mathbf{y}^{(c)}$ denotes the complementary solution, which satisfies the homogeneous equation

$$
-\frac{1}{\lambda} \frac{d \mathbf{y}}{d x}+\left(\mathbf{A}_{0}+\frac{1}{\lambda} \mathbf{A}_{1}+\frac{1}{\lambda^{2}} \mathbf{A}_{2}\right) \mathbf{y}=\mathbf{0}
$$

In order to obtain a particular solution, it is assumed that $\mathbf{y}^{(p)}$ has the form

$$
\mathbf{y}^{(p)}=\lambda \Psi_{0}+\Psi_{1}+\frac{1}{\lambda} \Psi_{2}+\ldots
$$

where the vectors $\Psi_{0}, \Psi_{1}, \Psi_{2}, \ldots$ are unknown functions of the dimensionless coordinate $x$. The expansion (17) is then substituted into Eq. (11), which becomes

$$
\begin{aligned}
\lambda\left[A_{0} \Psi_{0}-b_{0}\right] & +\left[A_{0} \Psi_{1}+A_{1} \Psi_{0}-\Psi_{0}^{\prime}-b_{1}\right] \\
+ & \frac{1}{\lambda}\left[A_{0} \Psi_{2}+A_{1} \Psi_{1}+A_{2} \Psi_{0}-\Psi_{1}^{\prime}-b_{2}\right]+\ldots=0
\end{aligned}
$$

where the superscript ' denotes differentiation with respect to $x$.

In Eq. (18), the coefficients of each power of $\lambda$ must vanish indepen- . dently, thus the vectors $\Psi_{0}, \Psi_{1}, \Psi_{2}, \ldots$ are obtained by solving the system of equations

$$
\begin{aligned}
& A_{0} \Psi_{0}=b_{0} \\
& A_{0} \Psi_{1}=b_{1}+\Psi_{0}^{\prime}-A_{1} \Psi_{0} \\
& A_{0} \Psi_{2}=b_{2}+\Psi_{1}^{\prime}-A_{1} \Psi_{1}-A_{2} \Psi_{0}
\end{aligned}
$$


When the particular solution $y^{(p)}$ is determined in this manner, it is found that the bending moment component $y_{1}$ is of $O\left(\lambda^{-2}\right)$, but the horizontal resultant component $y_{2}$ is of $O(\lambda)$. This implies that the particular solution is essentially the membrane solution, since the bending stresses are negligible relative to the direct stresses. It is therefore not necessary to calculate the particular solution by means of Eq. (19), since the membrane solution can be obtained directly by integrating the equation of vertical ( $y$ direction) equilibrium.

\section{$\S 5$ Asymptotic Solutions for Edge Bending}

As mentioned in the previous section, the complementary solution $y^{(c)}$ must satisfy the homogeneous equation (16). The complementary solution is taken in the form

$$
\mathbf{y}^{(c)}=e^{\lambda \xi}\left[\Phi_{0}+\frac{1}{\lambda} \Phi_{1}+\frac{1}{\lambda^{2}} \Phi_{2}+\ldots\right]
$$

where $\Phi_{0}, \Phi_{1}, \Phi_{2}, \ldots$, and $\xi(x)$ are unknown functions of $x$ which are independent of the large parameter $\lambda$. The exponential form (20) allows for rapid variation with $x$ near the edge of the shell. This is appropriate for the representation of edge bending, which is the physical phenomenon described by the complementary solution.

Substituting Eq. (20) into Eq. (16) gives the following eigenvalue problem (associated with the leading term in $\lambda$ ):

$$
\left[A_{0}-\xi^{\prime} I\right] \Phi_{0}=0
$$

in which I represents the identity mintrix. Setting the determinant of the coefficient matrix $\left[A_{0}-\xi^{\prime} I\right]$ equal to zero yields the characteristic equation

$$
\left(\sqrt{\frac{r_{2} c}{r_{2 e} c_{e}}} \xi^{\prime}\right)^{4}-2 \rho\left(\sqrt{\frac{r_{2} c}{r_{2 e} c_{e}}} \xi^{\prime}\right)^{2}+1=0
$$

where $\rho$ is the pressurization parameter defined by Eq. (10).

The characteristic equation (22), evaluated at $x=x_{e}$, becomes

$$
\alpha^{4}-2 \rho_{e} \alpha^{2}+1=0
$$

where

$$
\begin{aligned}
\alpha & =\xi^{\prime}\left(x_{e}\right) \\
\rho_{e} & =\rho\left(x_{e}\right)=\left.\rho\right|_{\text {g=o. }}
\end{aligned}
$$

Equation (23) has four roots, given by

$$
\alpha= \pm \sqrt{\rho_{e} \pm \sqrt{\rho_{e}^{2}-1}}
$$


Equation (21) provides the corresponding eigenvector, which is

$$
\Phi_{0}\left(x_{e}\right)=C\left\{\begin{array}{c}
-\alpha^{3} \\
1 \\
-\alpha^{2} \\
\alpha
\end{array}\right\}
$$

where $C$ is an arbitrary multiplicative constant.

For the purposes of this report, attention will be restricted to the oneterm approximation

$$
\mathbf{y}^{(c)} \cong e^{\lambda \xi} \Phi_{0}
$$

in which the terms in Eq. (20) of $O\left(\lambda^{-1}\right)$ or smaller are neglected. This approximation is accurate provided that:

(i) $\lambda$ is sufficiently large compared to $\rho_{e}$ and unity.

(ii) $\lambda \tan \phi_{e}$ is sufficiently large compared to unity.

Condition (i) is the main requirement for the validity of the asymptotic solution, and condition (ii) assures that the $O\left(\lambda^{-1}\right)$ term is negligible.

Also, because the edge bending effects are local to the edge zone, the functions $\xi(\boldsymbol{x})$ and $\boldsymbol{\Phi}_{0}(x)$ can be approximated by the leading terms of their Taylor series expansions in $x$, and Eq. (27) becomes

$$
y^{(c)} \cong e^{\lambda\left[\xi\left(x_{e}\right)+x \xi^{\prime}\left(x_{e}\right)\right]} \Phi_{0}\left(x_{e}\right)=C e^{\lambda \alpha\left(x-x_{e}\right)}\left\{\begin{array}{c}
-\alpha^{3} \\
1 \\
-\alpha^{2} \\
\alpha
\end{array}\right\}
$$

where Eqs. (24a) and (26) have been employed in order to express the solution in terms of the eigenvalue $\alpha$.

From the expression (25) for the four eigenvalues (roots of Eq. [23]), three cases can be identified:

(i) $\rho_{e} \leq-1$ : The roots are purely imaginary. The solutions (28) are sinusoidal in the meridional direction, which implies local buckling (with the bifurcation point $\rho_{e}=-1$ ).

(ii) $-1<\rho_{e}<1$ : The roots are complex. The solutions oscillate, but decay in the meridional direction. This is the typical edge bending behavior of a shell of revolution.

(iii) $\rho_{e} \geq 1$ : The roots are real, and the solutions decay exponentially in the meridional direction. This characterizes the edge bending behavior of a shell of revolution in high tension.

This report focuses on case (iii) above, which is appropriate for states of relatively high membrane tension due to pressure loads. In this case, Eq. (25) provides two positive real roots and two negative real roots.

If the dimensionless arc length coordinate $x$ is defined such that $x=$ 0 at $r=0$ (see Fig. 1), then the two positive roots correspond to two solutions of the form (28) which decay as $x$ decreases. For a shell with a 
single continuous edge (located at $x=x_{e}$ ), the complementary solution can therefore be written

$$
y^{(c)}=C_{1} e^{\lambda \alpha_{1}\left(x-x_{s}\right)}\left\{\begin{array}{c}
-\alpha_{1}^{3} \\
1 \\
-\alpha_{1}^{2} \\
\alpha_{1}
\end{array}\right\}+C_{2} e^{\lambda \alpha_{2}\left(x-x_{s}\right)}\left\{\begin{array}{c}
-\alpha_{2}^{3} \\
1 \\
-\alpha_{2}^{2} \\
\alpha_{2}
\end{array}\right\}
$$

where

$$
\begin{aligned}
& \alpha_{1}=\sqrt{\rho_{e}-\sqrt{\rho_{e}^{2}-1}} \\
& \alpha_{2}=\sqrt{\rho_{e}+\sqrt{\rho_{e}^{2}-1}}
\end{aligned}
$$

are the two positive eigenvalues, and $C_{1}$ and $C_{2}$ are multiplicative constants which must be determined by enforcing two boundary conditions at the edge of the shell.

The two negative roots of Eq. (23) correspond to edge effect solutions which decay as the dimensionless arc length coordinate $x$ increases. These solutions describe the bending response at an inner edge, if one exists. In this report, attention is focused on shells with one edge (no cutouts); thus the complementary solution is taken in the form (29).

It is frequently informative to compute the decay distances of the two exponential terms of the complementary solution (29). The decay distances $\delta_{1}$ and $\delta_{2}$, defined by

$$
\begin{aligned}
& \delta_{1}=\pi \sqrt{r_{2 e} c_{e}} / \alpha_{1} \\
& \delta_{2}=\pi \sqrt{r_{2 e} c_{e}} / \alpha_{2}
\end{aligned}
$$

are the distances along the meridian over which the exponential envelopes of the edge effects decay to about $4 \%$ of the maximum values.

For $\rho_{e} \gg 1$, Eqs. (31) can be approximated by

$$
\begin{aligned}
& \delta_{1} \cong \pi \sqrt{2 \rho_{e} T_{2 e} c_{e}} \\
& \delta_{2} \cong \pi \sqrt{\frac{r_{2 e} c_{e}}{2 \rho_{e}}}
\end{aligned}
$$

from which it is apparent that the first term of the solution (29) corresponds to an edge effect with decay distance $\delta_{1} \gg \sqrt{r_{2 e} c_{e}}$, while the second term corresponds to an edge effect with decay distance $\delta_{2} \ll \sqrt{r_{2 e} c_{e}}$. Note that without the pressurization effect $\left(\left|\rho_{e}\right| \ll 1\right)$, the two edge bending solutions have decay distances $\delta=O\left(\sqrt{r_{2 e} c_{e}}\right)$. With a large pressurization effect $\left(\rho_{e} \gg 1\right)$, the shell is in a state of high membrane tension, and the edge bending behavior is described by one solution which decays comparatively slowly along the meridian, and another which decays comparatively rapidly. 


\section{§6 Final Form of the Total Solution}

This section summarizes the results of the preceding two sections, and presents the total solution in a form which can be readily applied to problems where the shell is in a high state of tension due to pressure loading (i.e., for $\rho_{e}>1$ ). In view of Eq. (15), the total solution can be written

$$
\begin{gathered}
\left\{\begin{array}{c}
M_{\phi} \\
H \\
\chi \\
h
\end{array}\right\}=\left\{\begin{array}{c}
0 \\
H^{(m)} \\
\chi^{(m)} \\
h^{(m)}
\end{array}\right\}+C_{1} \exp \left[\frac{\alpha_{1}\left(s-s_{e}\right)}{\sqrt{r_{2 e} c_{e}}}\right]\left[\begin{array}{c}
-(E t c)_{e} \alpha_{1}^{3} \\
\frac{(E t)_{e}}{\lambda \sin \phi_{e}} \\
-\lambda \alpha_{1}^{2} \\
r_{e} \alpha_{1}
\end{array}\right\} \\
+C_{2} \exp \left[\frac{\alpha_{2}\left(s-s_{e}\right)}{\sqrt{r_{2 e} c_{e}}}\right]\left\{\begin{array}{c}
-(E t c)_{e} \alpha_{2}^{3} \\
\frac{(E t)_{e}}{\lambda \sin \phi_{e}} \\
-\lambda \alpha_{2}^{2} \\
r_{e} \alpha_{2}
\end{array}\right\}
\end{gathered}
$$

in which the first term is the membrane (particular) solution, denoted by. the superscripts ${ }^{(m)}$. Note that $M_{\phi}^{(m)}=0$, because the membrane solution has no appreciable bending stresses. The second two exponential terms are the edge bending (complementary) solution, obtained from Eqs. (29) and (14).

In Eq. (33), the quantities with subscript $e$ are evaluated at the edge $s=s_{e}$, and the quantities $\lambda, \alpha_{1}$, and $\alpha_{2}$ can be evaluated from the previously recorded equations (13), (30), (24b), and (10). In order to employ the solution (33), the following steps are required:

(i) Calculate the membrane solution. The forces and displacements of the membrane solution can be obtained directly from the basic equilibrium, constitutive, and kinematic relations.

From vertical equilibrium:

$$
V^{(m)}=\frac{p r}{2}
$$

from which it follows that

$$
\begin{aligned}
& H^{(m)}=V^{(m)} \cot \phi=\frac{p r}{2} \cot \phi \\
& N_{\phi}^{(m)}=\frac{V^{(m)}}{\sin \phi}=\frac{p r}{2 \sin \phi}=\frac{p r_{2}}{2}
\end{aligned}
$$


From normal equilibrium:

$$
N_{\theta}^{(m)}=-\frac{r_{2}}{r_{1}} N_{\phi}^{(m)}+r_{2} p=\frac{p r_{2}}{2}\left(2-\frac{r_{2}}{r_{1}}\right)
$$

From the stress-strain relations and Eqs. (34c) and (35):

$$
\begin{aligned}
& \varepsilon_{\phi}^{(m)}=\frac{N_{\phi}^{(m)}-\nu N_{\theta}^{(m)}}{E t}=\frac{p r_{2}}{2 E t}\left(1-2 \nu+\nu \frac{r_{2}}{r_{1}}\right) \\
& \varepsilon_{\theta}^{(m)}=\frac{N_{\theta}^{(m)}-\nu N_{\phi}^{(m)}}{E t}=\frac{p r_{2}}{2 E t}\left(2-\frac{r_{2}}{r_{1}}-\nu\right)
\end{aligned}
$$

where $\varepsilon_{\phi}$ and $\varepsilon_{\theta}$ are the midsurface strains in the meridional and circumferential directions.

From the kinematic relations:

$$
\begin{aligned}
h^{(m)} & =r \varepsilon_{\theta}=\frac{p r_{2}^{2} \sin \phi}{2 E t}\left(2-\frac{r_{2}}{r_{1}}-\nu\right) \\
\chi^{(m)} & =\cot \phi \varepsilon_{\phi}^{(m)}-\frac{1}{\sin \phi} \frac{d h}{d s}(m) \\
& =-\frac{p r_{2}}{2 E t}\left\{\left(\frac{r_{2}}{r_{1}}\right)^{2} \frac{d r_{1}}{d s}+\cot \phi\left[2\left(\frac{r_{2}}{r_{1}}\right)^{2}-5 \frac{r_{2}}{r_{1}}+3\right]\right\} \\
v & =\int_{s_{e}}^{0}\left(\chi \cos \phi+\varepsilon_{\phi} \sin \phi\right) d s \\
& =\int_{s_{e}}^{0}\left[V\left(\frac{1-\nu^{2}}{E t} \sin ^{2} \phi\right)+H\left(\frac{1-\nu^{2}}{E t} \sin \phi \cos \phi\right)\right. \\
& \left.\quad+\chi \cos \phi-h\left(\frac{\nu \sin \phi}{r}\right)\right] d s
\end{aligned}
$$

Equations (34b), (37b), and (37a) provide the membrane contributions $H^{(m)}, \chi^{(m)}$, and $h^{(m)}$ in Eq. (33).

(ii) Evaluate the constants $C_{1}$ and $C_{2}$. This is accomplished by enforcing two boundary conditions at $s=s_{\mathrm{e}}$. For a simply supported edge, the two conditions are $M_{\phi}=h=0$, and for a built-in edge, the conditions are $\chi=h=0$. The results are

$$
\text { Simply supported edge : }\left\{\begin{array}{l}
C_{1}=\frac{\alpha_{2}^{2} h_{e}^{(m)}}{r_{e} \alpha_{1}\left(\alpha_{1}^{2}-\alpha_{2}^{2}\right)} \\
C_{2}=\frac{\alpha_{1}^{2} h_{e}^{(m)}}{r_{e} \alpha_{2}\left(\alpha_{2}^{2}-\alpha_{1}^{2}\right)}
\end{array}\right.
$$




$$
\text { Built-in edge : }\left\{\begin{array}{l}
C_{1}=\frac{\alpha_{2} h_{e}^{(m)} / r_{e}+\chi_{e}^{(m)} / \lambda}{\alpha_{1}\left(\alpha_{1}-\alpha_{2}\right)} \\
C_{2}=\frac{\alpha_{1} h_{e}^{(m)} / r_{e}+\chi_{e}^{(m)} / \lambda}{\alpha_{2}\left(\alpha_{2}-\alpha_{1}\right)}
\end{array}\right.
$$

in which $h_{e}^{(m)}$ and $\chi_{e}^{(m)}$ are the horizontal displacement and meridional rotation of the membrane solution at the edge $s=s_{e}$.

\section{§7 Example: Paraboloidal Shell under Pressure Load}

Consider the parabolic shell of revolution depicted in Fig. 3. The edges of the shell are either simply supported $\left(M_{\phi}=h=0\right)$ or built-in $(\chi=h=$ 0 ), and the shell is subjected to a uniform pressure load. The equation of the meridian is given by

$$
y=\frac{r^{2}}{4 f}
$$

where $f$ is the focal length of the paraboloid. Equations (1), (2),(3), and (40) can be used to obtain the following expressions for the radii of curvature:

$$
\begin{aligned}
& r_{1}=2 f / \cos ^{3} \phi \\
& r_{2}=2 f / \cos \phi
\end{aligned}
$$

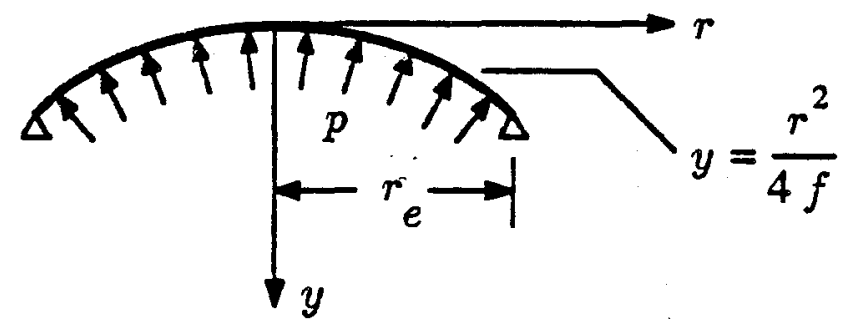

Figure 3. Paraboloidal shell with focal length $f$, subjected to a uniform pressure load $p$. The meridian curve is the parabola $y=r^{2} /(4 f)$.

The numerical calculations in this section utilize the geometry data, material properties, and pressure load given in Table 1. Substituting from Table 1 into Eqs. (1), (41b), (10), and (13) yields

$$
\begin{aligned}
\phi_{e} & =\tan ^{-1}\left(\frac{r_{e}}{2 f}\right) \cong 0.395 \\
r_{2 e} & =2 f / \cos \phi_{e} \cong 19.5 \mathrm{~m} \\
\rho_{e} & =\frac{p r_{2 e}^{2}}{4 E t c} \cong 46.6 \\
\lambda & =\left(r_{2 e} / c_{e}\right)^{\frac{1}{2}} \cong 504
\end{aligned}
$$


Because $\lambda \tan \phi_{e} \gg 1$ and $\lambda \gg \rho_{e}$, the one-term asymptotic solution should be reasonably accurate (see $\S 5$ ).

Table 1. Specifications for paraboloid, from Murphy (1987).

\begin{tabular}{|l|c|c|}
\hline \multicolumn{1}{|c|}{ Geometry } & Material Properties & Load \\
\hline$t=0.254 \mathrm{~mm}$ & $E=209 \mathrm{GPa}$ & $p=2000 \mathrm{~Pa}$ \\
$r_{e}=7.5 \mathrm{~m}$ & $\nu=0.3$ & \\
$f=9 \mathrm{~m}$ & & \\
\hline
\end{tabular}

\subsection{The Membrane Solution}

The membrane solution for the paraboloid is calculated by means of the equations in step (i) of $\$ 6$. Equations (34) can be used directly, and Eqs. (35-37a) are readily evaluated by substituting the radii of curvature given by Eqs. (41). In order to evaluate Eq. (37b), the relations

$$
\begin{aligned}
r_{2} / r_{1} & =\cos ^{2} \phi \\
\frac{d r_{1}}{d s} & =3 \tan \phi
\end{aligned}
$$

for the parabola are useful.

The results are

$$
\left\{\begin{array}{c}
M_{\phi}^{(m)} \\
H^{(m)} \\
\chi^{(m)} \\
h^{(m)}
\end{array}\right\}=\left\{\begin{array}{c}
0 \\
p f \\
-\frac{p f}{E t} \sin \phi\left(4-\sin ^{2} \phi\right) \\
\frac{2 p f^{2} \tan \phi}{E t \cos \phi}\left(2-\cos ^{2} \phi-\nu\right)
\end{array}\right\} \quad(44 a, b, c, d)
$$

where the angle $\phi$ is utilized as the independent variable (see Fig. 1). Note that for the parabola, the angle $\phi$, radial distance $r$, and meridional arc length $s$ are each acceptable independent variables, because the meridian curve is a single valued function of $\phi, r$, or $s$.

Also, from Eqs. (34c) and (35), the membrane stress resultants for the paraboloid are given by

$$
\begin{aligned}
& N_{\phi}^{(m)}=p f / \cos \phi \\
& N_{\theta}^{(m)}=p f\left(2-\cos ^{2} \phi\right) / \cos \phi
\end{aligned}
$$

The integral (37c) for the vertical displacement can be carried out to obtain the following expression for $v^{(m)}$ in terms of $\phi$ :

$$
v^{(m)}=\left.\frac{2 p f^{2}}{E t}\left(\cos \phi-\frac{3-\nu}{\cos \phi}+\frac{1-2 \nu}{3 \cos ^{3} \phi}\right)\right|_{\phi .} ^{\phi}
$$




\subsection{Evaluation of the Total Solution and Discussion of Results}

In this subsection, the total solution (33) is evaluated and compared with finite element results from Murphy (1987). From Eqs. (44c,d) and Table 1,

$$
\begin{aligned}
& \chi_{e}^{(m)}=-\frac{p f}{E t \sin \phi_{e}}\left(3-2 \cos ^{2} \phi_{e}-\cos ^{4} \phi_{e}\right) \cong-5.02 \times 10^{-4} \\
& h_{e}^{(m)}=\frac{2 p f^{2} \tan \phi_{e}}{E t \cos \phi_{e}}\left(2-\cos ^{2} \phi_{e}-\nu\right) \cong 2.34 \times 10^{-3} \mathrm{~m}
\end{aligned}
$$

And from Eqs. (30) and (42c),

$$
\begin{aligned}
& \alpha_{1}=\sqrt{\rho_{e}-\sqrt{\rho_{e}^{2}-1}} \cong 0.104 \\
& \alpha_{2}=\sqrt{\rho_{e}+\sqrt{\rho_{e}^{2}-1}} \cong 9.65
\end{aligned}
$$

From the results (47) and (48), the constants $C_{1}$ and $C_{2}$ in Eq. (33) can now be evaluated as

$$
\begin{array}{r}
\text { Simply supported edge : }\left\{\begin{array}{l}
C_{1} \cong-3.01 \times 10^{-3} \\
C_{2} \cong 3.72 \times 10^{-9}
\end{array}\right. \\
\text { Built-in edge : }\left\{\begin{array}{l}
C_{1} \cong-3.04 \times 10^{-3} \\
C_{2} \cong 3.50 \times 10^{-7}
\end{array}\right.
\end{array}
$$

where the expressions (38) and (39) are utilized. The results (42), (48), (49), (50), and Table 1 provide the numerical values which are required in order to evaluate the total solution (33).

In Eq. (33), the argument of the exponential factors is the meridional arc length $s$. A convenient approximation to the arc length in the edge zone is

$$
s-s_{e} \cong\left(r-r_{e}\right) / \cos \phi_{e}
$$

Equation (51) enables the exponential factors in Eq. (33) to be written in terms of the radial distance $r$, and is sufficiently accurate for calculations based on the one-term approximation (28).

Substituting Eq. (51) into the solution (33) provides an expression for the bending moment $M_{\phi}$ in terms of $r$, and the bending stress $\sigma_{\phi \mathrm{B}}(r)$ in the meridional direction is subsequently obtained as

$$
\begin{aligned}
\sigma_{\phi \mathrm{B}}=\frac{6 M_{\phi}}{t^{2}}=-E \sqrt{\frac{3}{1-\nu^{2}}}\left\{C_{1} \alpha_{1}^{3} \exp \left[\frac{\alpha_{1}\left(r-r_{e}\right)}{\cos \phi_{e} \sqrt{r_{2 e} c_{e}}}\right]\right. \\
\left.+C_{2} \alpha_{2}^{3} \exp \left[\frac{\alpha_{2}\left(r-r_{e}\right)}{\cos \phi_{e} \sqrt{r_{2 e} c_{e}}}\right]\right\}
\end{aligned}
$$


The decay distances of the two exponentially decaying contributions, calculated from Eqs. (31), are

$$
\begin{aligned}
& \delta_{1}=\pi \sqrt{r_{2 e} c_{e}} / \alpha_{1} \cong 1.17 \mathrm{~m} \\
& \delta_{2}=\pi \sqrt{r_{2 e} c_{e}} / \alpha_{2} \cong 0.0126 \mathrm{~m}
\end{aligned}
$$

where $\delta_{1}$ and $\delta_{2}$ are the distances along the meridian over which the edge bending stresses decay to about $4 \%$ of the maximum values at $r=r_{e}$.

Consider first the case of a simply supported edge. Figure 4 shows the bending stress $\sigma_{\phi B}$ as a function of the radial distance $r$. The first exponential term of Eq. (52), with decay distance $\delta_{1} \cong 1.17 \mathrm{~m}$, contributes the slowly decaying component of the edge effect (see Fig. 4). The second exponential term, with decay distance $\delta_{2} \cong 0.0126 \mathrm{~m}$, is responsible for the abrupt change near the edge $r=7.5 \mathrm{~m}$. For the simply-supported case, $\sigma_{\phi \mathrm{B}}$ must equal zero at the edge; thus the contributions of the two exponential terms in Eq. (52) cancel at $r=r_{e}$.

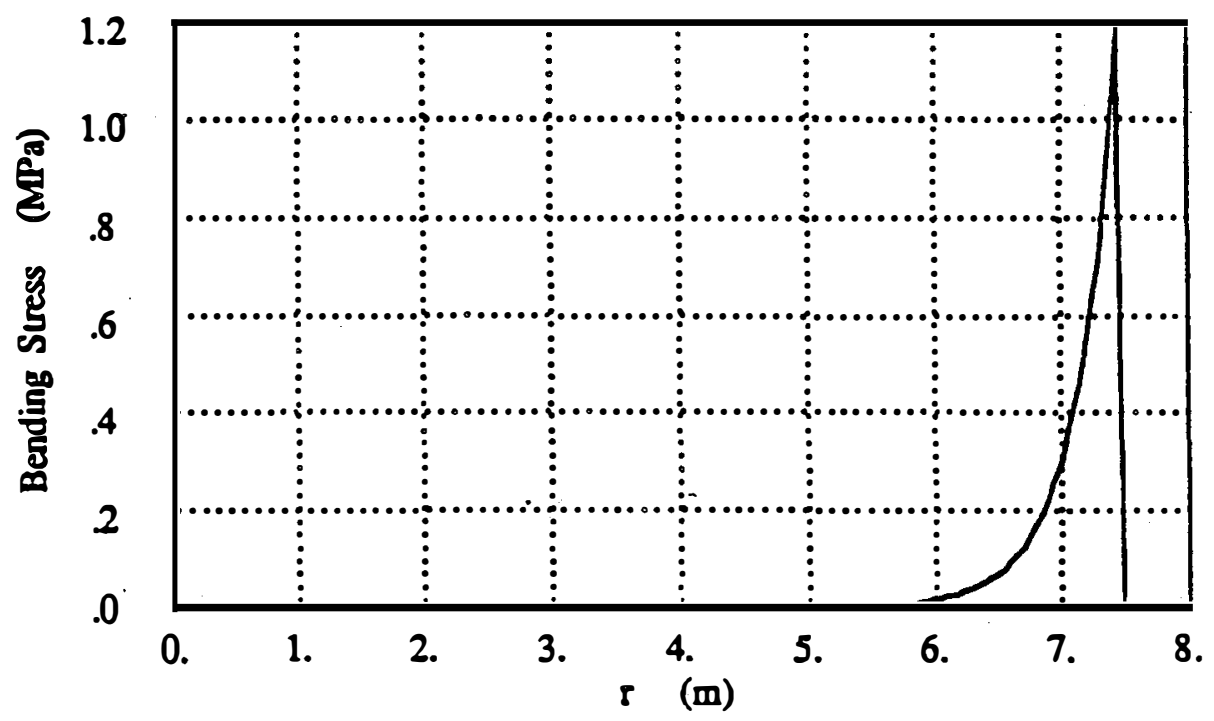

Figure 4. Asymptotic solution for the meridional bending stress in a simply supported parabolic shell under pressure load. The bending stress $\sigma_{\phi \mathrm{B}}$ is shown as a function of the radial distance $r$.

The variation of the bending stress in the region $7.3 \mathrm{~m} \leq r \leq 7.5 \mathrm{~m}$ (very close to the edge) is plotted in Fig. 5. Figure 5a shows the bending stress $\sigma_{\phi \mathrm{B}}$ for the case of a simply supported edge; the $\sim 10 \mathrm{~mm}$ decay distance of the second exponential term of Eq. (52) is clearly visible. Figure 5b shows the bending stress for the case of a built-in edge. The built-in edge condition gives rise to very high bending stresses at the edge, but these stresses are negligible a few centimeters away from the edge (see Fig. 5 b). 


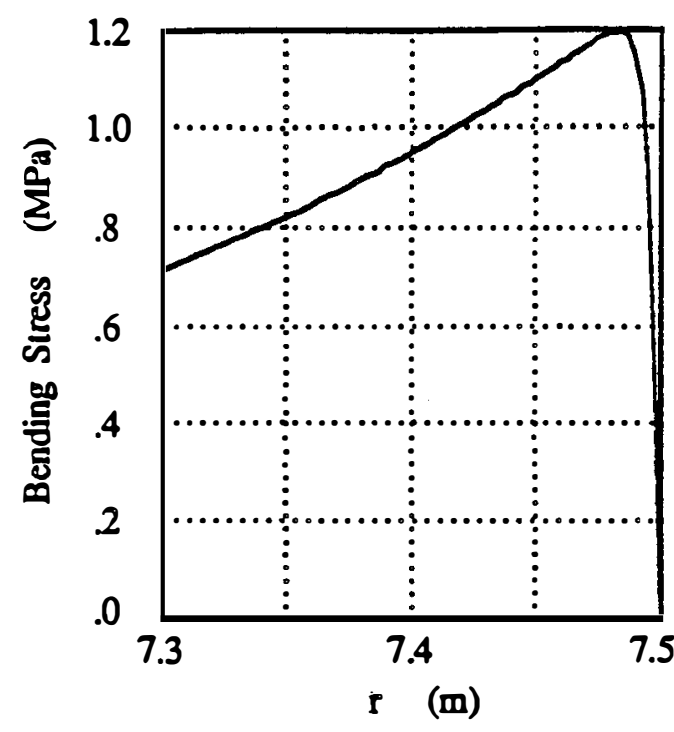

(a)

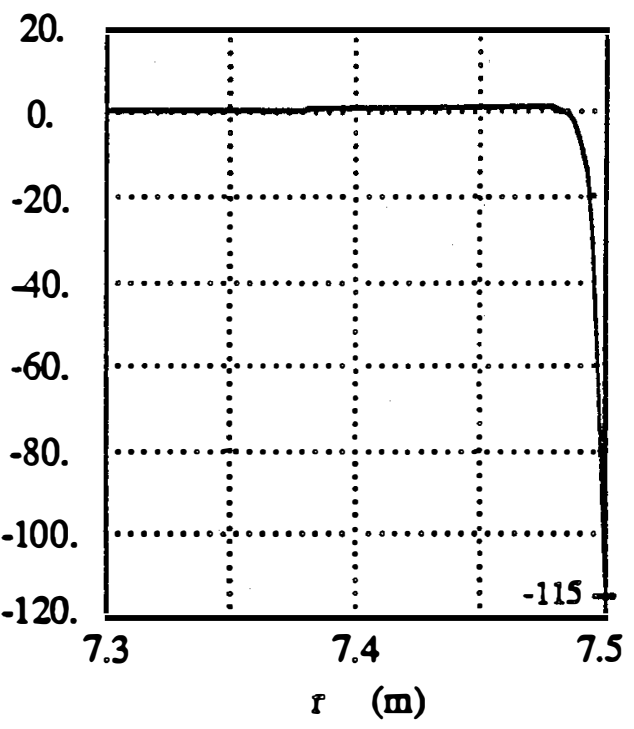

(b)

Figure 5. Asymptotic solution for the meridional bending stress $\sigma_{\phi \mathrm{B}}$ near the edge of the parabolic shell under pressure load. (a) Simply supported edge: the bending stress is zero at the edge $r=7.5 \mathrm{~m}$. (b) Built-in edge: the bending stress reaches a maximum value at the edge.

The rotation $\chi$ of the meridian, from Eq. (33), is given by

$$
\chi=\chi^{(m)}-\lambda \alpha_{1}^{2} C_{1} \exp \left[\frac{\alpha_{1}\left(r-r_{e}\right)}{\cos \phi_{e} \sqrt{r_{2 e} c_{e}}}\right]-\lambda \alpha_{2}^{2} C_{2} \exp \left[\frac{\alpha_{2}\left(r-r_{e}\right)}{\cos \phi_{e} \sqrt{r_{2 e} c_{e}}}\right]
$$

in which $\chi^{(m)}$ is evaluated from Eq. (44c). Figure 6 shows the rotation $\chi(r)$ for the case of a simply supported edge. The solid line represents the asymptotic result (54), and the cross marks represent the values of the rotation from the finite element analysis by Murphy (1987) (see Fig. 6). The two approaches both show the $\sim 1 \mathrm{~m}$ decay distance of the edge effect, but good quantitative agreement is not obtained near the edge (see Fig. 6).

The variation of the meridional rotation in the region $7.3 \mathrm{~m} \leq r \leq 7.5 \mathrm{~m}$ is plotted in Fig. 7. Figure 7a shows the meridional rotation $\chi$ for the case of a simply supported edge; Figure $7 \mathrm{~b}$ shows the meridional rotation for the case of a built-in edge. Note that the rotation of the meridian is essentially the same for both edge conditions, except in the "boundary layer" region a few centimeters from the edge. In order to enforce the built-in edge condition, the second, rapidly decaying, exponential term of Eq. (52) participates significantly (see Fig. 7b).

The meridional stress resultant $N_{\phi}$ is given by Eq. (7a), in which $H$ is obtained from Eq. (33), and $V$ is obtained from Eq. (34a). Note that the vertical component of the complementary solution must be zero in order to satisfy vertical equilibrium; thus $V=V^{(m)}=p r / 2$. 


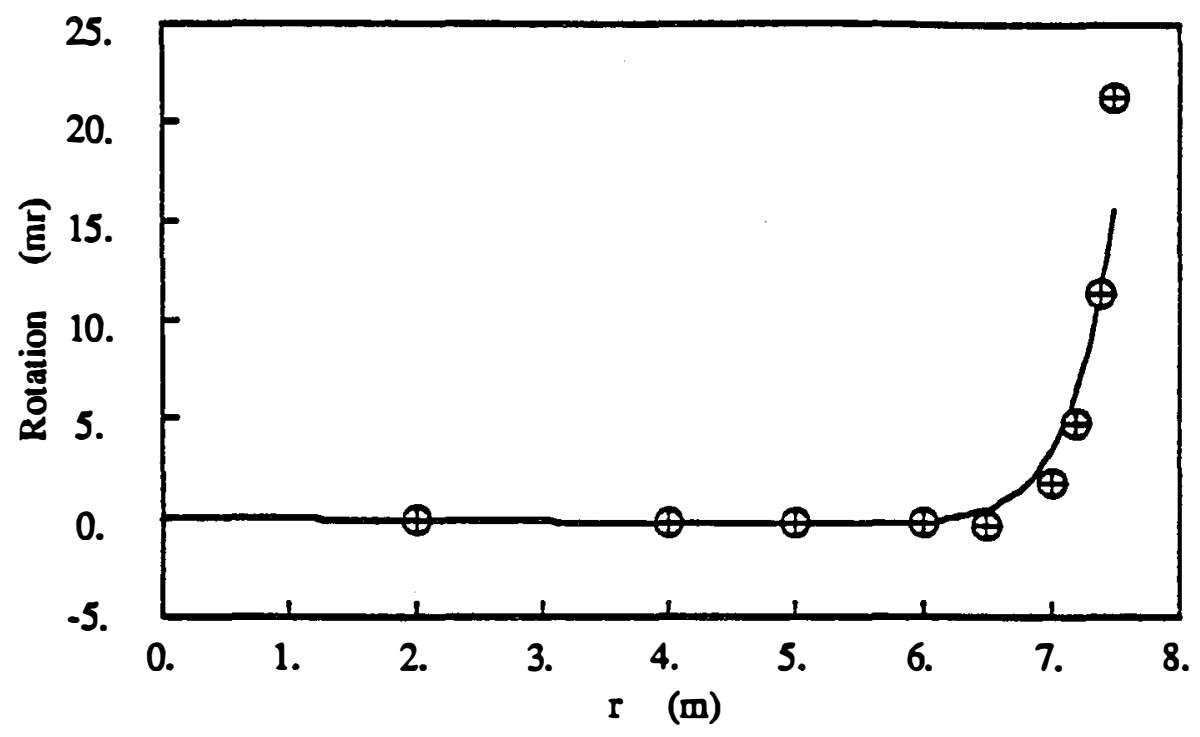

Figure 6. Rotation $\chi$ as a function of radial distance $r$ for the (1) simply supported parabolic shell under pressure load. The solid line is the asymptotic solution; cross marks are ANSYS results from Murphy (1987).

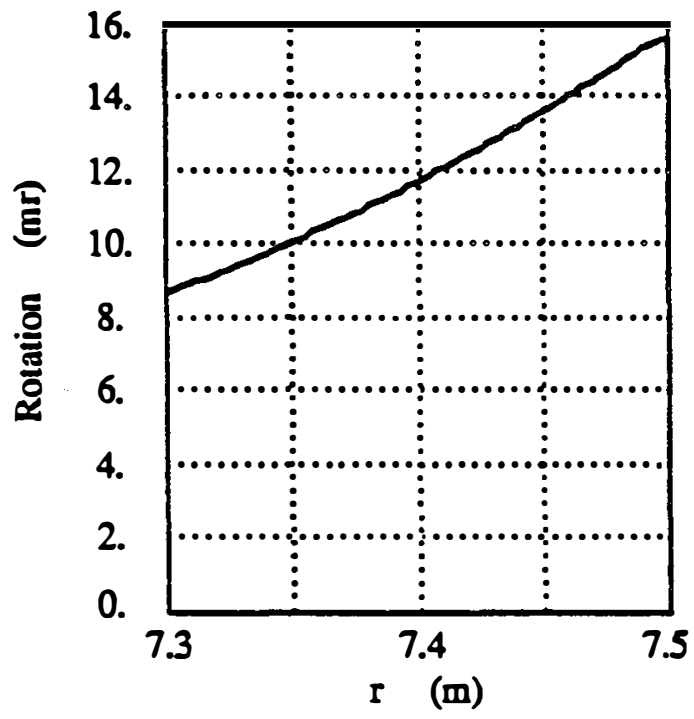

(a)

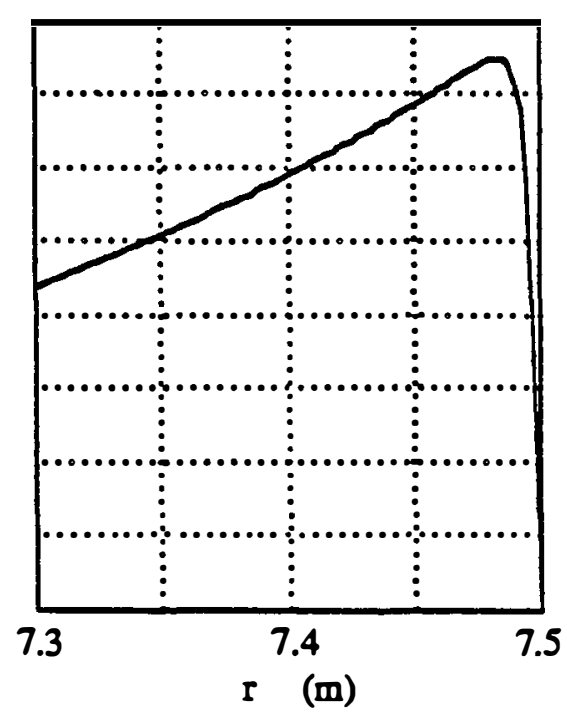

(b)

Figure 7. Asymptotic solution for the meridional rotation $\chi$ near the edge of the parabolic shell under pressure load. (a) Simply supported edge: the meridional rotation reaches a maximum value at the edge $r=7.5 \mathrm{~m}$. (b) Built-in edge: the meridional rotation is zero at the edge.

The meridional and circumferential strains $\varepsilon_{\phi}$ and $\varepsilon_{\theta}$ can now be obtained from the relations 


$$
\begin{aligned}
\varepsilon_{\phi} & =\frac{1-\nu^{2}}{E t} N_{\phi}-\nu \varepsilon_{\theta} \\
\varepsilon_{\theta} & =h / r
\end{aligned}
$$

where $h$ is given by Eq. (33). The circumferential stress resultant $N_{\theta}$ can be calculated by substituting Eqs. (55) into the constitutive relation

$$
N_{\theta}=\frac{E t}{1-\nu^{2}}\left(\varepsilon_{\theta}+\nu \varepsilon_{\phi}\right)
$$

For the case of a simply supported edge, the stress resultants $N_{\phi}$ and $N_{\theta}$ given by Eqs. (7a) and (56) are plotted in Fig. 8 (solid lines). Figure 8 also shows the values for the stress resultants from the finite element analysis by Murphy (1987) (circles), which agree well with the asymptotic solution. Note that the meridional stress resultant $N_{\phi}$ remains approximately constant along the meridian, but the circumferential stress resultant $N_{\theta}$ decreases considerably near the edge (see Fig. 8). For the case of a built-in (or clamped) edge (not shown in Fig. 8), the stress resultants $N_{\phi}$ and $N_{\theta}$ differ only slightly (less than $1 \%$ ) from their values for the simply supported case.

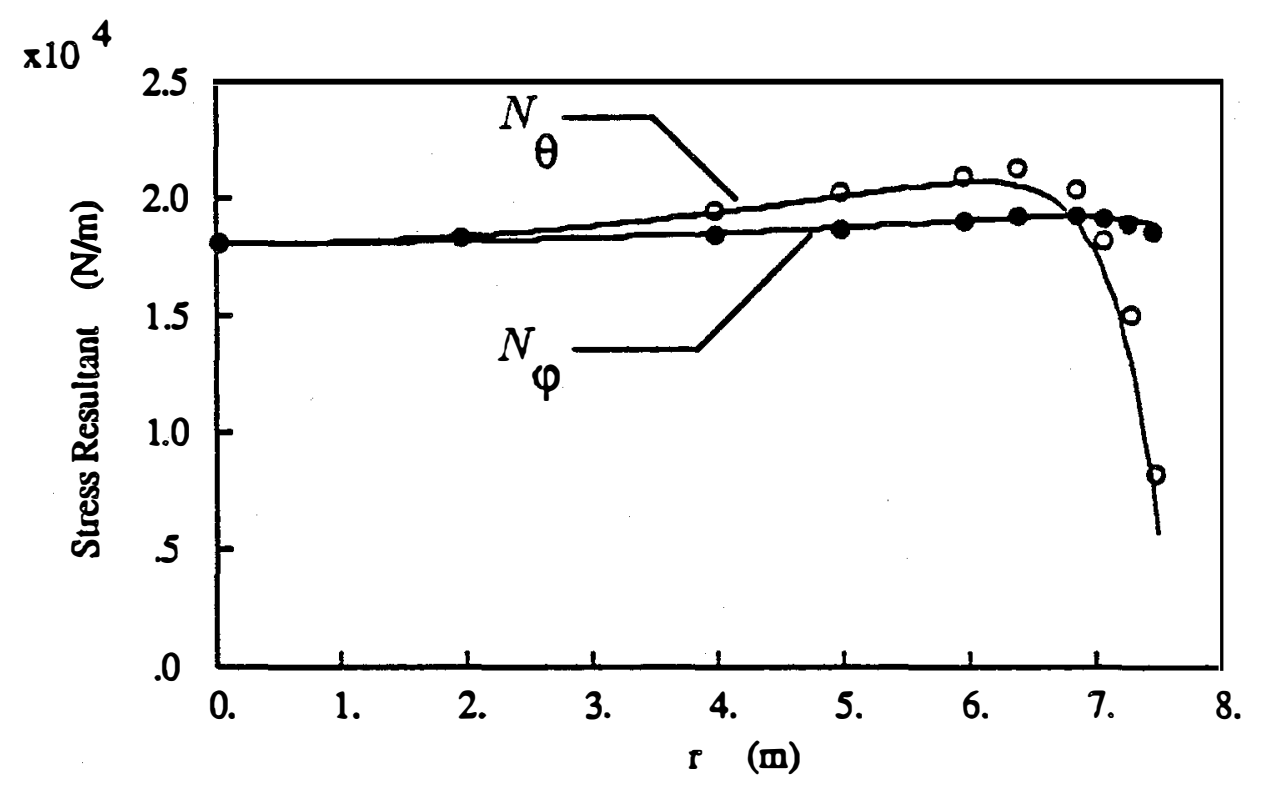

Figure 8. Stress resultants $N_{\phi}$ and $N_{\theta}$ for the simply supported parabolic shell under pressure load. Solid lines are the asymptotic solution; circles are ANSYS results from Murphy (1987).

The horizontal displacement $h$, from Eq. (33), is given by

$$
h=h^{(m)}+r_{e} \alpha_{1} C_{1} \exp \left[\frac{\alpha_{1}\left(r-r_{e}\right)}{\cos \phi_{e} \sqrt{r_{2 e} c_{e}}}\right]+r_{e} \alpha_{2} C_{2} \exp \left[\frac{\alpha_{2}\left(r-r_{e}\right)}{\cos \phi_{e} \sqrt{r_{2 e} c_{e}}}\right]
$$


in which $h^{(m)}$ is evaluated from Eq. (44d).

The vertical displacement $v$ can be decomposed into the contributions from the membrane and complementary solutions:

$$
v=v^{(m)}+v^{(c)}
$$

where $v^{(m)}$ is given by Eq. (46). The vertical displacement $v^{(c)}$ of the complementary solution can be obtained by evaluating an integral of the form $(37 c)$. Because $V^{(c)}=0$, the integral $(37 c)$ for $v^{(c)}$ becomes

$$
v^{(c)}=\int_{s .}^{s}\left[H^{(c)}\left(\frac{1-\nu^{2}}{E t} \sin \phi \cos \phi\right)+\chi^{(c)} \cos \phi-h^{(c)}\left(\frac{\nu \sin \phi}{r}\right)\right] d s
$$

Substituting the complementary part (exponential terms) of the solution (33) into Eq. (59) yields

$$
\begin{aligned}
v^{(c)}=\int_{s_{e}}^{s}\left\{C_{1} \exp \left[\frac{\alpha_{1}\left(s-s_{e}\right)}{\sqrt{r_{2 e} c_{e}}}\right]\right. & {\left[\frac{(E t)_{e}}{\lambda \sin \phi_{e}}\left(\frac{1-\nu^{2}}{E t} \sin \phi \cos \phi\right)\right.} \\
& \left.-\lambda \alpha_{1}^{2} \cos \phi-r_{e} \alpha_{1}\left(\frac{\nu \sin \phi}{r}\right)\right] \\
+C_{2} \exp \left[\frac{\alpha_{2}\left(s-s_{e}\right)}{\sqrt{r_{2 e} c_{e}}}\right] & {\left[\frac{(E t)_{e}}{\lambda \sin \phi_{e}}\left(\frac{1-\nu^{2}}{E t} \sin \phi \cos \phi\right)\right.} \\
& \left.\left.-\lambda \alpha_{2}^{2} \cos \phi-r_{e} \alpha_{2}\left(\frac{\nu \sin \phi}{r}\right)\right]\right\} d s
\end{aligned}
$$

Although Eq. (60) appears complicated, the integral can be simplified by noting that it is of the form

$$
\int_{x_{e}}^{x} f(x) e^{\lambda x} d x=\left.\frac{1}{\lambda} f(x) e^{\lambda x}\right|_{x_{e}} ^{x}+O\left(\lambda^{-2}\right)
$$

where $\lambda$ is large compared to unity and $f(x)$ and its derivatives are independent of $\lambda$. Equation (61), which follows from integration by parts, can be utilized in evaluating the integral (60). The result is

$$
\begin{array}{r}
v^{(c)=}\left\{C _ { 1 } \frac { \sqrt { r _ { 2 e } c _ { e } } } { \alpha _ { 1 } } \operatorname { e x p } [ \frac { \alpha _ { 1 } ( r - r _ { e } ) } { \operatorname { c o s } \phi _ { e } \sqrt { r _ { 2 e } c _ { e } } } ] \left[\frac{1-\nu^{2}}{\lambda \sin \phi_{e}} \sin \phi \cos \phi\right.\right. \\
\left.-\lambda \alpha_{1}^{2} \cos \phi-r_{e} \alpha_{1}\left(\frac{\nu \sin \phi}{r}\right)\right] \\
+C_{2} \frac{\sqrt{r_{2 e} c_{e}}}{\alpha_{2}} \exp \left[\frac{\alpha_{2}\left(r-r_{e}\right)}{\cos \phi_{e} \sqrt{r_{2 e} c_{e}}}\right]\left[\frac{1-\nu^{2}}{\lambda \sin \phi_{e}} \sin \phi \cos \phi\right. \\
\left.\left.-\lambda \alpha_{2}^{2} \cos \phi-r_{e} \alpha_{2}\left(\frac{\nu \sin \phi}{r}\right)\right]\right\}\left.\right|_{r_{e}} ^{r}
\end{array}
$$


where terms of $O\left(\lambda^{-2}\right)$ are neglected, and Eq. (51) is employed in order to express $v^{(c)}$ in terms of $r$.

The horizontal displacement $h$, from Eq. (57), and the vertical displacement $v$, from Eq. (58), are plotted in Fig. 9 (solid lines), for the case of a simply supported edge. Figure 9 also shows values of the horizontal and vertical displacement from the finite element solution by Murphy (1987) (cross marks). The finite element values for the horizontal displacement $h$ agree well with the asymptotic solution. Near the axis of the paraboloid, however, the magnitude of the vertical displacement $v$ given by the finite element solution is greater than that of the asymptotic solution (see Fig. 9).

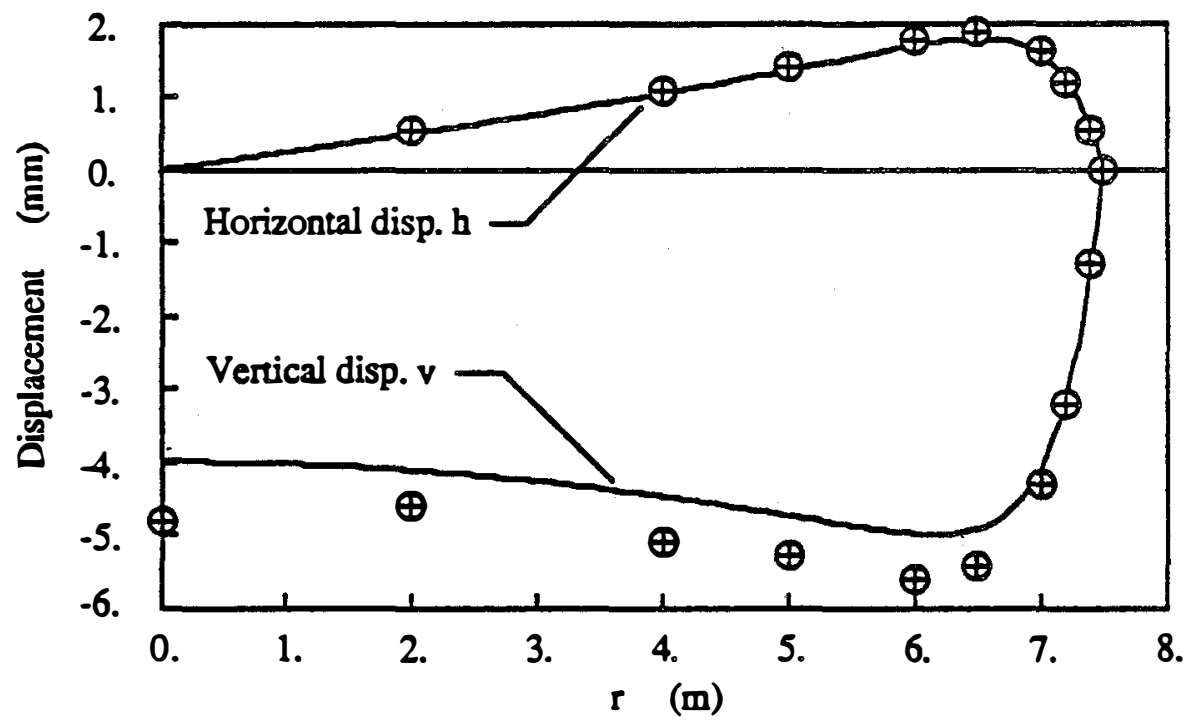

Figure 9. Horizontal displacement $h$ and vertical displacement $v$ for the simply supported parabolic shell under pressure load. Solid lines are the asymptotic solution; cross marks are ANSYS results from Murphy (1987).

Note that the only difference between the solutions for the simply supported and built-in edge conditions is the equation (Eq. [38 ] or Eq. [39]) which is employed in the evaluation of the constants $C_{1}$ and $C_{2}$. For the case of a built-in edge (not shown in Fig. 9), the displacements $h$ and $v$ differ only slightly (less than 1\%) from their values for the simply supported case. 


\section{References}

Murphy, L. M. (1987). Private communication from the Solar Energy Research Institute, May 8, 1987.

Ranjan, G. V. and Steele, C. R. (1980). "Nonlinear corrections for edge bending of shells," J. Appl. Mech., Vol. 47, pp. 861-865.

Steele, C. R. and Skogh, J. (1970). "Slope discontinuities in pressure vessels," J. Appl. Mech., Vol. 37, pp. 587-595. 


\section{Non-Axisymmetric Deformations of Thin Paraboloidal Shells with Initial Prestress}

submitted to the Solar Energy Research Institute by

C. D. Balch* and C. R. Steele§

Division of Applied Mechanics

Stanford University

Stanford, California 94305

SHELLTECH report 88-2, May 1988

* Research Associate

$\S$ Professor of Applied Mechanics 


\begin{abstract}
A tenth-order formulation is presented for non-axisymmetric deformations of shells of revolution. The formulation includes transverse shear deformation, as well as the nonlinear moderate rotation terms which become important in the presence of high initial prestress. Asymptotic solutions are then obtained for non-axisymmetric edge bending effects in a pressureloaded paraboloidal shell. It is shown that for thin shells, two types of edge effects may occur: one with a short decay distance relative to the square root of the radius to thickness ratio, and one with a long decay distance. For the membrane and inextensional behavior, solutions based on shallow shell theory are employed.

The asymptotic solutions and shallow shell results for the paraboloid have been incorporated into a FORTRAN computer program which runs in a few minutes or less on a PC. The analysis of a thin paraboloidal dish is discussed, and examples are presented which demonstrate the effect of prescribing circumferential harmonics of edge displacement, and the effect of an asymmetric pressure load.
\end{abstract}




\section{$\S 1$ Introduction}

The present investigation is concerned with non-arisymmetric deformations of shells of revolution, and is an extension of a previous report (Steele and Balch, 1987), in which asymptotic solutions for axisymmetric edge bending were discussed. In order to analyze general non-axisymmetric deformations, the circumferential dependence of the displacements and stresses is expressed as a Fourier series of circumferential barmonics. The shell equations are derived from a mixed variational principle of the Reissner type, which leads to a state vector (matrix) form of the equations for the $n$th circumferential barmonic.

The general response of a shell to prescribed edge deformations and applied loads consists of a combination of membrane, inextensional, and edge bending behavior. Membrane behavior is characterized by direct stresses which are much greater that any bending stresses, while inextensional behavior is characterized by direct stresses which are negligible relative to the bending stresses. Edge bending effects involve coupled membrane and bending behavior and are typically local to the edge zone, decaying rapidly with increasing distance from the edge of the shell.

In the present work, asymptotic solutions to the shell equations for the nth circumferential barmonic are obtained using the asymptotic expansion discussed by Steele (1980). These solutions describe edge bending, and are valid for edge effects with decay distances which are small in comparison to the shell radii of curvature, and for perturbations from a nominal state of membrane prestress. Solutions for membrane and inextensional behavior are obtained independently, using shallow shell theory.

The analysis of the paraboloidal dish collector discussed by Kutscher et. al. (1988) is the primary motivation and concern of the present work. The membrane, inextensional, and edge bending solutions are therefore derived for the paraboloidal shell. These solutions have been incorporated into a FORTRAN computer program which can be run in a few minutes on a PC. The output of the program consists of the displacements, surface rotations, and stresses at a predetermined set of grid points on the shell. Several examples are discussed which illustrate the response of a paraboloidal shell to non-axisymmetric edge conditions and loading. For the 
analysis of asymmetric pressure loading, a "wind load" membrane solution with $\cos \theta(n=1)$ circumferential dependence is employed.

\section{§2 Reference Coordinate System and Notation}

Figure 1 shows an element of a shell of revolution. The radial, circumferential, and axial coondinates $(r, \theta, z)$ are used.

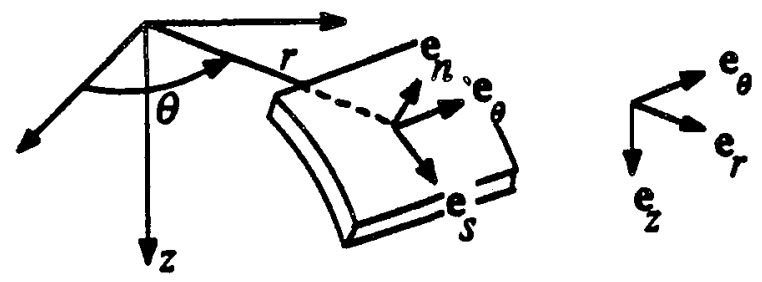

Figure 1. Shell element, coordinate system, and reference basis vectors.

Figure 1 also shows the local basis vectors $\left\{\mathbf{e}_{9}, \mathbf{e}_{\theta}, \mathbf{e}_{n}\right\}$ in the meridional, circumferential, and normal directions. The stress and moment resultants which act on the shell element are commonly expresed relative to this basis. Figure 2 shows the stress and moment resultants and their directions of positive action.
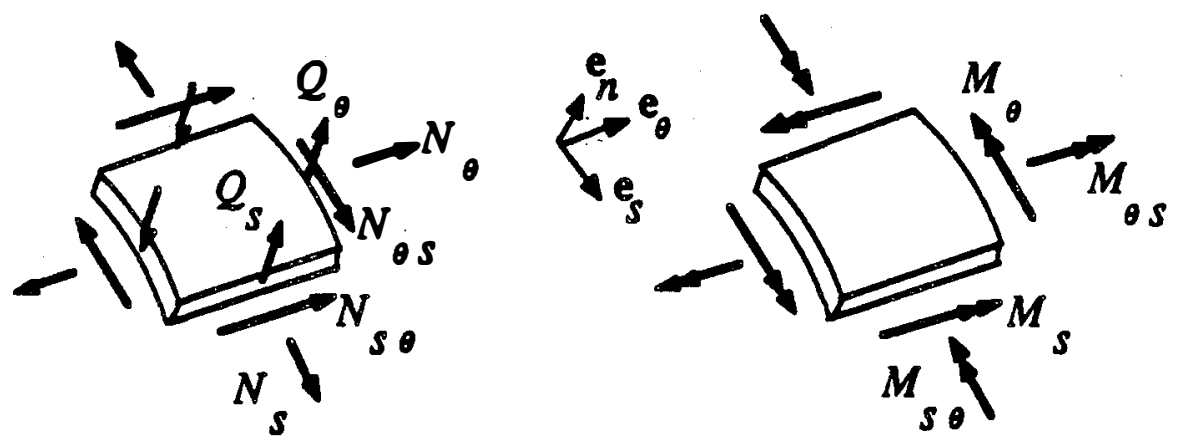

Figure 2. Stress and moment resultants acting on a shell element.

For some purposes, it is convenient to utilize the basis $\left\{\mathbf{e}_{r}, \mathbf{e}_{\boldsymbol{z}}, \mathbf{e}_{\theta}\right\}$ shown in Fig. 1, and to decompose the stress resultants and displacements in terms of radial and vertical components:

$$
\begin{aligned}
& N_{r}=N_{\mathrm{s}} \cos \phi+Q_{\mathrm{s}} \sin \phi \\
& N_{z}=N_{\mathrm{s}} \sin \phi-Q_{\mathrm{s}} \cos \phi
\end{aligned}
$$




$$
\begin{aligned}
& u_{r}=u_{0} \cos \phi+u_{n} \sin \phi \\
& u_{z}=u_{0} \sin \phi-u_{n} \cos \phi
\end{aligned}
$$

where $N$ and $u$ denote stress resultants and midsurace displacements in the directions indicated by the subscripts, and the angle $\phi$, given by

$$
\phi=\tan ^{-1}\left(\frac{d z}{d r}\right)
$$

is the angle between the normal to the meridian and the $z$ axis (see Fig. 3).

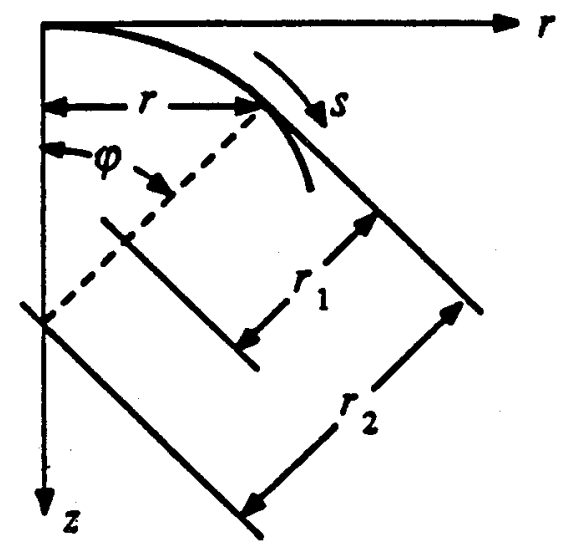

Figure 3. Meridian of a shell of revolution and meridional arclength coordinate $s$. The distances $r_{1}$ and $r_{2}$ are the radii of curvature in the meridional and circumferential directions.

The principal kinematic degrees of freedom to be considered are depicted in Fig. 4. The two rotation angles $\chi_{(\bullet, \theta)}$ of the normal to the midsurface are considered in addition to the three midsurface displacements $u_{(r, z, \theta)}$. This Reissner-type of kinematic approximation incorporates the effect of transverse shear deformation.

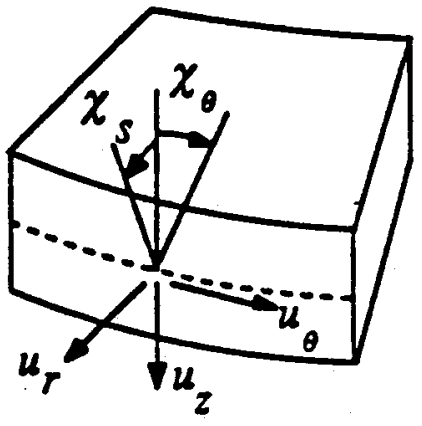

Figure 4. The kinematic degrees of freedom along an edge $s=$ const. consist of the radial, vertical, and circumferential displacements $u_{r}, u_{z}, u_{\theta}$ and the meridional and circumferential rotation angles $\chi_{0}, \chi_{\theta}$ of the normal to the midsurface. 
As a consequence of these kinematics, a complete specification of the boundary conditions requires the enforcement of five conditions along the edge of the shell. Either a displacement quantity or its corresponding force quantity may be prescribed. Along an edge of constant $s$, the fire force quantities which correspond to the five kinematic degrees of freedom shown in Fig. 4 are the two moment resultants $M_{0}$ and $M_{0 \theta}$ and the three stress resultants $N_{p,}, N_{z}$, and $N_{\rho \theta}$.

\section{§3 Formulation of the Shell Equations}

The governing equations for the shell of revolution can be derived by applying a mixed variational principle of the Reissner form, which can be written

$$
\begin{aligned}
& \delta \Pi=\delta \int_{0}^{2 \pi} \int_{01}^{\rho_{2}}\left\{N_{\rho} \varepsilon_{0}+N_{\theta} \varepsilon_{\theta}+2 N_{\rho \theta} \varepsilon_{0 \theta}\right. \\
& +M_{0} \kappa_{0}+M_{\theta} \kappa_{\theta}+2 M_{0 \theta} \kappa_{\theta \theta}+Q_{0}\left(\chi_{0}-\beta_{0}\right)+Q_{\theta}\left(\chi_{\theta}-\beta_{\theta}\right) \\
& -\frac{1}{2 E t}\left[N_{\theta}^{2}-2 \nu N_{0} N_{\theta}+N_{\theta}^{2}+2(1+\nu) N_{\theta \theta}^{2}\right] \\
& -\frac{6}{E t^{3}}\left[M_{\theta}^{2}-2 \nu M_{0} M_{\theta}+M_{\theta}^{2}+2(1+\nu) M_{\theta \theta}^{2}\right] \\
& \left.-\frac{\mu}{2 E t}\left(Q_{\theta}^{2}+Q_{\theta}^{2}\right)-p_{s} u_{s}-p_{\theta} u_{\theta}-p_{n} u_{n}\right\} r d s d \theta=0 \text {. }
\end{aligned}
$$

where $\varepsilon_{\rho}, \varepsilon_{\theta}$, and $\varepsilon_{\rho \theta}$ are the midsurface strains; $\kappa_{\rho}, \kappa_{\theta}$, and $\kappa_{\rho \theta}$ are the midsurface curvature changes; $\beta$, and $\beta_{\theta}$ are the midsurface rotations; $P(\theta, \theta, n)$ are the components of the distributed load; $\nu$ is Poisson's ratio; and the shear flexibility factor $\mu$ is given by

$$
\mu \approx 12(1+\nu) / 5
$$

for isotropic materials.

Because the shell kinematics involve five degrees of freedom, it is possible to write Eq. (4) in terms of the five displacement quantities shown in Fig. 4 and the five corresponding force quantities. This leads to a modified form of Eq. (4) in terms of ten independent unknowns. The algebra required to achieve this can be carried out systematically by means of matrix operations. The symbolic manipulation program MACSYMA (1983) was utilized to derive and check the results.

First, consider the constitutive relations for linear elastic behavior:

$$
\left[\begin{array}{l}
F_{1} \\
F_{2}
\end{array}\right]=\left[\begin{array}{ll}
\Gamma_{11} & \Gamma_{12} \\
\Gamma_{21} & \Gamma_{22}
\end{array}\right]\left[\begin{array}{l}
D_{1} \\
D_{2}
\end{array}\right]
$$


in which the vectors $F_{(1,2)}$ and $D_{(1,2)}$ are defined by

$$
\begin{array}{ll}
F_{1}=\left[\begin{array}{c}
M_{s} \\
M_{s \theta} \\
Q_{s} \\
N_{s} \\
N_{s \theta}
\end{array}\right] \quad ; \quad \mathrm{D}_{1}=\left[\begin{array}{c}
\kappa_{s} \\
2 \kappa_{s \theta} \\
\chi_{s}-\beta_{s} \\
\varepsilon_{s} \\
2 \varepsilon_{s \theta}
\end{array}\right] \\
\mathbf{F}_{2}=\left[\begin{array}{c}
M_{\theta} \\
Q_{\theta} \\
N_{\theta}
\end{array}\right] \quad ; & \mathrm{D}_{2}=\left[\begin{array}{c}
\kappa_{\theta} \\
\chi_{\theta}-\beta_{\theta} \\
\varepsilon_{\theta}
\end{array}\right]
\end{array}
$$

and the symmetric matrix $\Gamma$ is given in Appendix $A$ for the case of isotropic behavior. The matrix relation (6) can be partially inverted to obtain

$$
\left[\begin{array}{l}
D_{1} \\
F_{2}
\end{array}\right]=\left[\begin{array}{ll}
\Phi_{11} & \Phi_{12} \\
\boldsymbol{\Phi}_{21} & \boldsymbol{\Phi}_{22}
\end{array}\right]\left[\begin{array}{l}
D_{1} \\
D_{2}
\end{array}\right]
$$

where

$$
\begin{aligned}
& \Phi_{11}=\Gamma_{11}^{-1} \\
& \Phi_{12}=-\Gamma_{11}^{-1} \Gamma_{12}=-\Phi_{21}^{T} \\
& \Phi_{22}=\Gamma_{22}-\Gamma_{21} \Gamma_{11}^{-1} \Gamma_{12}
\end{aligned}
$$

Making use of the definitions $(7,8)$ and Eq. (9), it is possible to rewrite the variational equation (4) as

$$
\begin{aligned}
\delta \Pi= & \delta \int_{0}^{2 \pi} \int_{0_{1}}^{\Delta_{2}}\left[F_{1}^{T}\left(D_{1}+\Phi_{21}^{T} D_{2}\right)+\frac{1}{2} D_{2}^{T} \Phi_{22} D_{2}\right. \\
& \left.-\frac{1}{2} F_{1}^{T} \Phi_{11} F_{1}-D^{T} P\right] r d s d \theta=0
\end{aligned}
$$

where the superscript ${ }^{T}$ denotes transposition. In Eq. (11), the dependent force quantities $F_{2}$ have been eliminated from the integrand, and the vectors

$$
\mathbf{D}=\left[\begin{array}{l}
\chi_{\theta} \\
\chi_{\theta} \\
u_{r} \\
u_{z} \\
u_{\theta}
\end{array}\right] \quad ; \quad \mathbf{P}=\left[\begin{array}{c}
0 \\
0 \\
p_{r} \\
p_{z} \\
p_{\theta}
\end{array}\right]
$$

in the final load term of the integrand are the displacement and load vectors relative to the $\left\{\mathbf{e}_{r}, \mathbf{e}_{z}, \mathbf{e}_{\theta}\right\}$ basis. 
The next objective is to express Eq. (11) in terms of the five displacement quantities in the vector $\mathbf{D}$ and the five force quantitities in the vector F, defined by

$$
F=\left[\begin{array}{c}
r M_{0} \\
r M_{00} \\
r N_{r} \\
r N_{z} \\
r N_{\theta 0}
\end{array}\right]=r G_{1}^{T} F_{1}
$$

where the transformation matrix $G_{1}$ is

$$
G_{1}=\left[\begin{array}{ccccc}
1 & 0 & 0 & 0 & 0 \\
0 & 1 & 0 & 0 & 0 \\
0 & 0 & \sin \phi & -\cos \phi & 0 \\
0 & 0 & \cos \phi & \sin \phi & 0 \\
0 & 0 & 0 & 0 & 1
\end{array}\right]
$$

In order to express $D_{1}$ and $D_{2}$ in terms of the vector $D$, kinematic relations which relate strains and curvature changes to displacements and rotations are required. Consider a deformation with $\cos n \theta$ ciroumferential dependence, for which

$$
\begin{aligned}
& F_{1}=\left[\begin{array}{c}
M_{0}^{(n)} \cos n \theta \\
M_{\rho \theta}^{(n)} \sin n \theta \\
Q_{\rho}^{(n)} \cos n \theta \\
N_{g}^{(n)} \cos n \theta \\
N_{\rho \theta}^{(n)} \sin n \theta
\end{array}\right] \quad ; \quad D_{1}=\left[\begin{array}{c}
\kappa_{0}^{(n)} \cos n \theta \\
2 \kappa_{\rho 0}^{(n)} \sin n \theta \\
\left(\chi_{0}^{(n)}-\beta_{0}^{(n)}\right) \cos n \theta \\
\varepsilon_{\theta}^{(n)} \cos n \theta \\
2 \varepsilon_{\rho 0}^{(n)} \sin n \theta
\end{array}\right] \\
& F_{2}=\left[\begin{array}{c}
M_{\theta}^{(n)} \cos n \theta \\
Q_{\theta}^{(n)} \sin n \theta \\
N_{\theta}^{(n)} \cos n \theta
\end{array}\right] \quad ; \quad D_{2}=\left[\begin{array}{c}
\kappa_{\theta}^{(n)} \cos n \theta \\
\left(\chi_{\theta}^{(n)}-\beta_{\theta}^{(n)}\right) \sin n \theta \\
\epsilon_{\theta}^{(n)} \cos n \theta
\end{array}\right]
\end{aligned}
$$

and

$$
\mathbf{F}=\left[\begin{array}{l}
r M_{\theta}^{(n)} \cos n \theta \\
r M_{s \theta}^{(n)} \sin n \theta \\
r N_{r}^{(n)} \cos n \theta \\
r N_{z}^{(n)} \cos n \theta \\
r N_{\rho \theta}^{(n)} \sin n \theta
\end{array}\right] \quad ; \quad \mathbf{D}=\left[\begin{array}{l}
\chi_{a}^{(n)} \cos n \theta \\
\chi_{\theta}^{(n)} \sin n \theta \\
u_{(}^{(n)} \cos n \theta \\
u_{z}^{(n)} \cos n \theta \\
u_{\theta}^{(n)} \sin n \theta
\end{array}\right] ; \quad \mathbf{P}=\left[\begin{array}{c}
0 \\
0 \\
p_{r}^{(n)} \cos n \theta \\
p_{z}^{(n)} \cos n \theta \\
p_{\theta}^{(n)} \sin n \theta
\end{array}\right]
$$

where the superscript $(n)$ denotes a Fourier coefficient which is independent of $\theta$.

The kinematic relations given by Flügge (1973) can be written in the matrix form

$$
\begin{aligned}
& D_{1}^{(n)}=G_{1} \frac{d}{d s}^{(n)}+G_{2}^{(n)} D^{(n)} \\
& D_{2}^{(n)}=G_{3}^{(n)} D^{(n)}
\end{aligned}
$$


where $D^{(n)}, D_{1}^{(n)}$, and $D_{2}^{(n)}$ are the vectors of the Fourier coefficients in Eqs. (15b), (16b), and (17b), and the matrices $G_{2}^{(n)}$ and $G_{3}^{(n)}$ are given by

$$
\begin{aligned}
\mathbf{G}_{2}^{(n)} & =\left[\begin{array}{ccccc}
0 & 0 & 0 & 0 & 0 \\
\mp n / r & -\cos \phi / r & 0 & 0 & 0 \\
1 & 0 & 0 & 0 & 0 \\
0 & 0 & 0 & 0 & 0 \\
0 & 0 & \mp n \cos \phi / r . & \mp n \sin \phi / r & -\cos \phi / r
\end{array}\right] \\
\mathbf{G}_{3}^{(n)} & =\left[\begin{array}{ccccc}
\cos \phi / r & \pm n / r & 0 & 0 & 0 \\
0 & 1 & \mp n \sin \phi / r & \pm n \cos \phi / r & -\sin \phi / r \\
0 & 0 & 1 / r & 0 & \pm n / r
\end{array}\right]
\end{aligned}
$$

The upper sign of $\{ \pm, \mp\}$ in Eqs. (19) applies for the circumferential dependence represented by Eqs. (15-17), and the lower sign applies for $\sin n \theta$ dependence, which would be represented by Eqs. (15-17) with cosines and sines interchanged.

For the nth hamonic of circumferential variation, the integration with respect to $\theta$ in Eq. (11) just leads to an overall multiplicative factor of $\pi$ (or $2 \pi$, for the axisymmetric case $n=0$ ). Dividing through by this constant then leaves the variational equation

$$
\begin{gathered}
\delta I=\delta \int_{s_{2}}^{\theta_{2}}\left[F_{1}^{(n)^{T}}\left(D_{1}^{(n)}+\Phi_{21}^{T} D_{2}^{(n)}\right)+\frac{1}{2} D_{2}^{(n)^{T}} \Phi_{22} D_{2}^{(n)}\right. \\
\left.-\frac{1}{2} F_{1}^{(n)^{T}} \Phi_{11} F_{1}^{(n)}-D^{(n)^{T}} P^{(n)}\right] r d s=0
\end{gathered}
$$

in which the $\theta$ dependence has been eliminated, and $F_{1}^{(n)}$ and $P^{(n)}$ are the vectors of the Fourier coefficients in Eqs. (15a) and (17c).

Inverting Eq. (13) yields

$$
\mathbf{F}_{1}^{(n)}=\frac{1}{r} \mathbf{G}_{1} \mathbf{F}^{(n)}
$$

Substituting Eqs. (18) and (21) into Eq. (20) now provides the final variational equation in terms of $\mathbf{F}$ and $\mathbf{D}$ :

$$
\begin{gathered}
\delta \Pi=\delta \int_{0_{1}}^{0_{2}}\left(F^{(n)^{T}} \frac{d D^{(n)}}{d s}+D^{(n)^{T}} E^{(n)} F^{(n)}-\frac{1}{2} F^{(n)^{T}} \mathrm{C} F^{(n)}\right. \\
\left.+\frac{1}{2} D^{(n)^{T}} K^{(n)} D^{(n)}-D^{(n)^{T}} \mathrm{~B}^{(n)}\right) d s=0
\end{gathered}
$$

where the matrices $\mathbf{E}^{(n)}, C, \mathbf{K}^{(n)}$, and the load vector $B^{(n)}$ are given by

$$
\begin{aligned}
\mathbf{E}^{(n)^{T}} & =\mathbf{G}_{1}^{T}\left(G_{2}^{(n)}+\Phi_{21}^{T} G_{3}^{(n)}\right) \\
\mathbf{C} & =\frac{1}{r} G_{1}^{T} \Phi_{11} G_{1} \\
\mathbf{K}^{(n)} & =r G_{3}^{(n)^{T}} \Phi_{22} G_{3}^{(n)} \\
B^{(n)} & =r P^{(n)}
\end{aligned}
$$


The explicit forms of $E^{(n)}, C$, and $K^{(n)}$ for the case of isotropic material behavior are given in Appendix B. Note that the matrices $C$ and $K^{(n)}$ are symmetric.

As pointed out by Steele (1988) in deriving the fourth-order system for axisymmetric deformations, a variational principle of the form (22) leads to the self-adjoint system

$$
-\frac{d}{d s}\left[\begin{array}{l}
F \\
D
\end{array}\right]^{(n)}+\left[\begin{array}{cc}
E & K \\
C & -E^{T}
\end{array}\right]^{(n)}\left[\begin{array}{l}
F \\
D
\end{array}\right]^{(n)}=\left[\begin{array}{l}
B \\
0
\end{array}\right]^{(n)}
$$

The state vector equation (24) is a tenth-order system of governing equations for the nth Fourier harmonic. It will be seen that the form (24) of the shell equations is convenient for obtaining asymptotic solutions for edge effects.

\section{§4 Initial Tension Due to Pressure Load}

The nonlinear equations for finite axisymmetric deformations of shells of revolution were first derived by Reissner (1950). Asymptotic solutions to Reissner's equations are presented by Ranjan and Steele (1980). From these and other previous works, it is seen that "prestress," or initial membrane tension or compression, contributes an important geometric nonlinearity which can significantly affect the edge bending behavior of a shell.

For an analysis of a shell with prestress, the moderate rotation terms

$$
\frac{1}{2} N_{\ominus} \beta_{\ominus}^{2}+\frac{1}{2} N_{\theta} \beta_{\theta}^{2}+N_{\ominus \theta} \beta_{0} \beta_{\theta}
$$

must be added to the integrand in Eq. (4). For isotropic or transversely orthotropic materials, the constitutive relations

$$
\begin{aligned}
& Q_{0}=\frac{E t}{\mu}\left(\chi_{0}-\beta_{0}\right) \\
& Q_{0}=\frac{E t}{\mu}\left(\chi_{\theta}-\beta_{0}\right)
\end{aligned}
$$

can be used to express the moderate rotation terms (25) as

$$
\frac{1}{2} N_{0} \chi_{0}^{2}+\frac{1}{2} N_{\theta} \chi_{\theta}^{2}+N_{0 \theta \chi_{0} \chi_{\theta}}
$$

provided that

$$
\left\{N_{\theta}, N_{\theta}, N_{s \theta}\right\} \ll E t / \mu
$$

In general, the inclusion of the terms (27) in the integrand of Eq. (4) leads to a nonlinear system of governing equations. 
For the purposes of the present investigation, which concerns prestress due to pressure load, the nonlinear terms (27) are linearized to obtain

$$
\frac{1}{2}\left(\bar{N}_{\theta} \chi_{\theta}^{2}+\bar{N}_{\theta} \chi_{\theta}^{2}\right)
$$

where $\bar{N}$, and $\bar{N}_{\theta}$ denote the nominal state of membrane stress due to the pressure load. The quadratic terms (29) are the linearized strain energy terms associated with perturbations relative to the nominal state of tension. Note that the pressure load produces no membrane shear stress.

When the linearized moderate rotation terms (29) are added to the integrand of Eq. (4), the resulting system of equations (24) remains the same, except for the first two diagonal elements of the matrix $K^{(n)}$, which become

$$
\begin{aligned}
& \mathbf{K}_{11}^{(n)^{\prime}}=\mathbf{K}_{11}^{(n)}+r \bar{N}_{0} \\
& \mathbf{K}_{22}^{(n)^{\prime}}=\mathbf{K}_{22}^{(n)}+r \bar{N}_{\theta}
\end{aligned}
$$

The system of equations to be considered in the subsequent analysis is therefore Eq. (24) with the modifications (30).

\section{§5 Asymptotic Solutions}

For the analysis of edge effects, we consider the homogeneous case of Eq. (24), which can be written

$$
-\frac{d Y}{d s}+A Y=0
$$

where $Y$ is the unknown state vector and $A$ is the coefficent matrix which depends on the meridional coordinate $s$.

In order to obtain solutions to Eq. (31) which are of a relatively simple form, the formal asymptotic expansion introduced by Steele (1980) is employed. In this approach, the solution $Y$ is represented by the series

$$
\mathbf{Y}=\exp \left(\int \xi(s) d s\right)\left[Y_{0}(s)+Y_{1}(s)+\ldots\right]
$$

where $\xi(s)$ is an unknown function and the $Y_{i}$ are unknown vectors which are slowly varing functions of $s$. The form (32) is appropriate for edge effects which decay rapidly with increasing distance from the edge.

The expansion (32) satisfies the governing equations (31) if $\xi$ and the $Y_{i}$ satisfy the relations

$$
\begin{aligned}
& (A-I \xi) Y_{0}=0 \\
& (A-I \xi) Y_{i}=\frac{d Y_{i-1}}{d s} \quad(i \geq 1)
\end{aligned}
$$


where $I$ is the identity matrix. The function $\xi$ and vector $Y_{0}$ are therefore an eigenvalue and corresponding eigenvector of the coefficient matrix $A$. The $Y_{i}$ are determined recursively from Eq. (33b).

In the present work, as in the previous investigation (Steele and Balch, 1987), the one-term asymptotic solution is considered. This provides an approximate solution of simple exponential form:

$$
\mathrm{Y}(s) \approx C e^{\xi\left(\rho_{e}\right)\left(e-s_{e}\right)} Y_{0}\left(s_{e}\right)
$$

where $C$ is an arbitrary constant and $\xi\left(s_{e}\right)$ and $Y_{0}\left(s_{e}\right)$ are an eigenvalue and corresponding eigenvector evaluated at the edge $s=s_{\mathrm{e}}$. The one-term asymptotic solution therefore requires the calculation of the eigenvalues and eigenvectors of $\mathbf{A}\left(s_{e}\right)$.

Because the matrix $\mathbf{A}$ is non-symmetric and of tenth order, it has, in general, ten complex eigenvalues $\xi$, which satisfy the characteristic equation

$$
|\mathbf{A}-\mathbf{I} \xi|=0
$$

It can be shown, however, that if $\xi$ is an eigenvalue of a matrix which is of the form of the coefficient matrix in Eq. (24), then $-\xi$ is also an eigenvalue. The solutions which correspond to the five eigenvalues with positive real parts decay with decreasing $s$ (see Fig. 3), and represent edge effects at an outer edge. The solutions which correspond to the five eigenvalues with negative real parts decay with increasing $s$ and represent edge effects at an inner edge. For a shell with a single edge (no internal cutouts), we need therefore consider only the five edge effect solutions for the outer edge.

For qualitative discussion of the behavior of the edge effects, it is useful to define the decay distance $\delta$ by

$$
\delta=\pi /\left|\operatorname{Re}\left[\xi\left(s_{e}\right)\right]\right|
$$

which is the distance from the edge at which the envelope of the edge effect has decayed by about $4 \%$.

The five roots of Eq. (35) with positive real part can be determined numerically. For isotropic material behavior, the five roots can be categorized as follows:

(i) The largest root is of $O\left(t^{-1}\right)$, where $t$ is the shell thickness. This root corresponds to a very localized transverse shear edge effect with a decay distance $\delta=O(t)$.

(ii) The next two largest roots correspond to edge bending effects. Figure 5 shows the decay distances of the two edge bending solutions as a function of the dimensionless harmonic index

$$
\bar{n}=n \sqrt{2 r_{2} c} /(2 r)
$$


where $r_{2}$ is the circumferential radius of curvature and $c$ is the reduced thickoness, defined by

$$
c=t /\left[12\left(1-\nu^{2}\right)\right]^{\frac{1}{2}}
$$

where $t$ is the shell thickness and $\nu$ is Poisson's ratio.

For zero prestress (solid line in Fig. 5), the two edge bending solutions have equal decay distances $\delta=O\left(\sqrt{r_{2} t}\right.$ ). For high prestress (dashed and dotted lines in Fig. 5), one bending edge effect decays rapidly and one decays slowly relative to the bending edge effects without prestress. Initial membrane tension in the shell therefore affects the edge bending behavior dramatically, as pointed out in the previous report (Steele and Balch, 1987) for the case of axisymmetric deformations $(n=0)$.

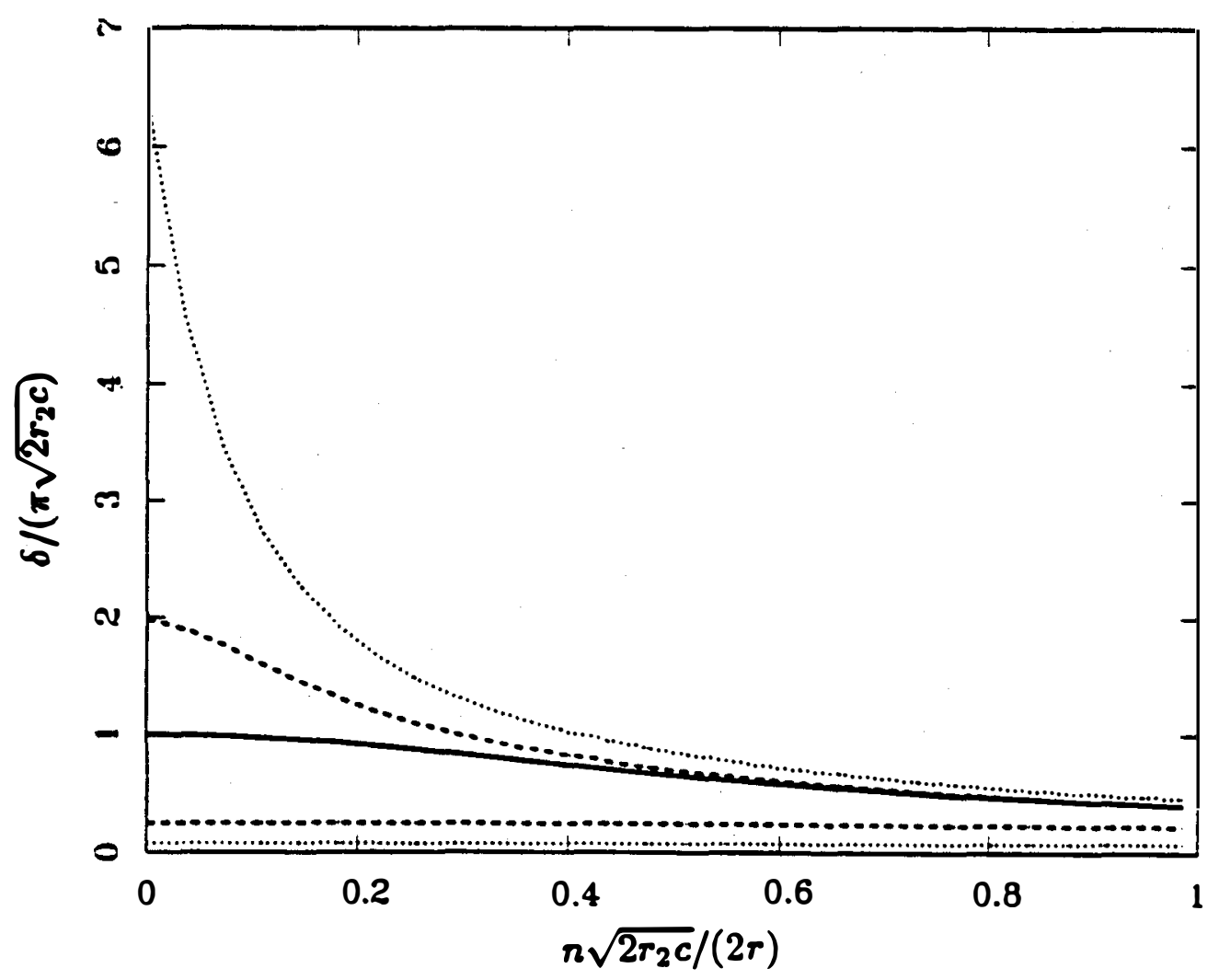

Figure 5. Decay distance $\delta$ as a function of $n$ for the two edge bending solutions, with nominal prestress $N_{2} r_{2} /(2 E t c)=$ $N_{0} r_{1} /(2 E t c)=0$ (solid line), 4 (dashed line), and 40 (dotted line). The longest decay distance occurs for the axisymmetric case $n=0$.

(iii) The lowest two roots of Eq. (35) correspond to a membrane solution, for which the direct stresses are much greater that the bending stresses, and an inextensional solution, for which the direct stresses are negligible relative to the bending stresses. With the present formulation, the asymptotic solution (34) provides accurate approximations to the membrane and 
inextensional solutions only for very high harmonics $\bar{n}=O(1)$, for which the response of the shell approaches that of a flat plate.

Because the lower barmonics are of particular interest in the present investigation, solutions valid for general $n$ based on shallow shell theory are employed to represent the membrane and inextensional response. This is discussed in the next section.

\section{\$6 Shallow Shell Solutions for the Paraboloid}

A shallow paraboloidal or spherical shell can be described by

$$
z(r)=r^{2} /(2 R)
$$

where $z(r)$ and $R$ are the axial coordinate and radius of curvature of the undeformed meridian.

For the paraboloid, the homogeneous form of the shallow shell equations, from Whassow (1958), p. 381, can be written with respect to the coordinates in Fig. 1 as follows:

$$
\begin{gathered}
\frac{1}{E t} \Delta \Delta \phi+\frac{1}{R} \Delta u_{z}=0 \\
E t c^{2} \Delta \Delta u_{z}-\bar{N} \Delta u_{z}-\frac{1}{R} \Delta \phi=0
\end{gathered}
$$

in which $E$ is the elastic modulus, $\bar{N}$ is the nominal state of membrane tension; $\phi$ is the Airy stress function for perturbations relative to this nominal state, i.e.,

$$
\begin{aligned}
& N_{r}=\bar{N}+\frac{1}{r} \frac{\partial \phi}{\partial r}+\frac{1}{r^{2}} \frac{\partial^{2} \phi}{\partial \theta^{2}} \\
& N_{\theta}=\bar{N}+\frac{\partial^{2} \phi}{\partial r^{2}} \\
& N_{s \theta}=\frac{\partial}{\partial r}\left(\frac{1}{r} \frac{\partial \phi}{\partial \theta}\right)
\end{aligned}
$$

and $\Delta$ is the harmonic differential operator defined by

$$
\Delta=\frac{\partial^{2}}{\partial r^{2}}+\frac{1}{r} \frac{\partial}{\partial r}+\frac{1}{r^{2}} \frac{\partial^{2}}{\partial \theta^{2}}
$$

Substituting the circumferential variation

$$
\phi=\phi^{(n)} \cos n \theta ; \quad u_{z}=u_{z}^{(n)} \cos n \theta
$$

into the equations (40) yields the coupled ODE's

$$
\begin{gathered}
\frac{1}{E t} \Delta_{n} \Delta_{n} \phi^{(n)}+\frac{1}{R} \Delta_{n} u_{z}^{(n)}=0 \\
E t c^{2} \Delta_{n} \Delta_{n} u_{z}^{(n)}-\bar{N} \Delta_{n} u_{z}^{(n)}-\frac{1}{R} \Delta_{n} \phi^{(n)}=0
\end{gathered}
$$


where

$$
\Delta_{n}=\frac{d^{2}}{d r^{2}}+\frac{1}{r} \frac{d}{d r}-\frac{n^{2}}{r^{2}}
$$

The solutions to Eqs. (44) can be separated into three families:

(i) Edge bending solutions, which satisfy

$$
\left(E_{t c}^{2} \Delta_{n} \Delta_{n}-\bar{N} \Delta_{n}+\frac{E t}{R^{2}}\right) u_{z}^{(n)}=0
$$

Since asymptotic solutions for edge bending were obtained in the previous section, the solutions to Eq. (46) will not be discussed.

(ii) Membrane solutions, which satisfy

$$
\Delta_{n} \phi^{(n)}=0
$$

(iii) Inextensional solutions, which satisfy

$$
\Delta_{n} u_{z}^{(n)}=0
$$

The membrane and inextensional solutions for the paraboloid are therefore simple barmonic functions, given by

$$
\begin{aligned}
& \phi^{(n)}=C_{1} r^{n}+C_{2} r^{-n} \\
& u_{z}^{(n)}=C_{1}^{0} r^{n}+C_{2}^{0} r^{-n}
\end{aligned}
$$

where $C_{1}, C_{2}, C_{1}^{\prime}$, and $C_{2}^{\prime}$ are arbitrary constants. For the present analysis, only the first terms in Eqs. (49) need be considered; the second terms are singular at $r=0$ and must be omitted for the case of a paraboloid with a single edge. From Eqs. (49), the constitutive relations (6,A.1-3), and the kinematic relations (18), all the components in the state vector $Y$ of Eq. (31) can be calculated.

The results for the membrane solutions are:

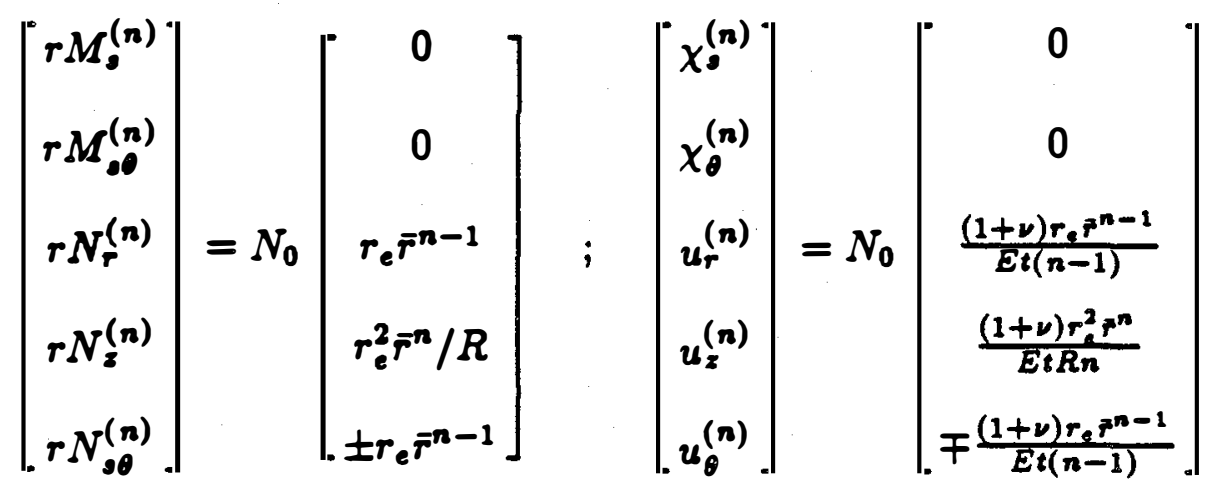

where $N_{0}$ is an arbitrary constant, $r_{e}$ is the radial coordinate of the edge, and $\bar{r}$, defined by

$$
\bar{r}=r / r_{e}
$$


is a dimensionless radial coordinate. In Eq. (50), the upper sign of $\{ \pm, \mp\}$ corresponds to $\cos n \theta$ circumferential dependence of $\phi^{(n)}$ and $u_{z}^{(n)}$, and the lower sign corresponds to $\sin n \theta$ circumferential dependence.

The results for the inextensional solutions are:

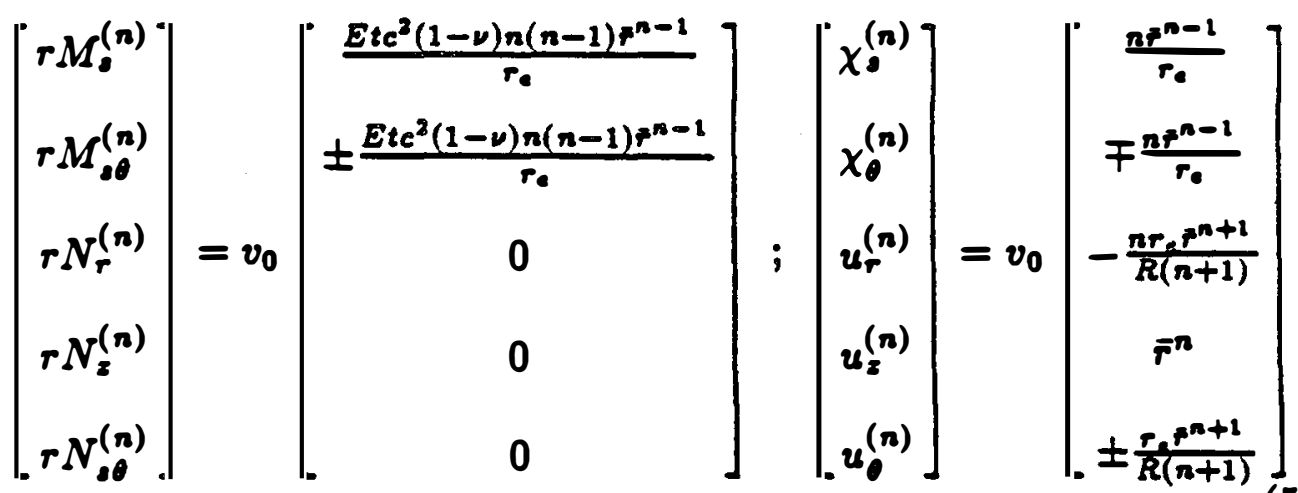

where $v_{0}$ is an arbitrary constant.

For the case $n=1$, the solutions (50) and (52) describe rigid body motions of the shell. Equations (50) and (52) provide the remaining solutions which are required for analyzing edge effects in a paraboloidal dish.

\section{\$7 Membrane Solutions for Asymmetric Pressure Load}

In this section, we discuss membrane solutions for a paraboloidal shell subjected to asymmetric pressure loading such that

$$
\begin{array}{rll}
p=p^{(n)} \cos n \theta & ; & N_{s}=N_{\rho}^{(n)} \cos n \theta \\
N_{0}=N_{\theta}^{(n)} \cos n \theta & ; & N_{\rho 0}=N_{\rho \theta}^{(n)} \sin n \theta
\end{array}
$$

where $p$ is the pressure in the $e_{n}$ direction in Fig. 1. The primary concern of the present analysis is the "wind load" distribution which corresponds to the $n=1$ harmonic.

For a shallow paraboloidal shell, the membrane equations of equilibrium can be written

$$
\begin{gathered}
\frac{d}{d s}\left(r N_{+}\right)+(1+n) N_{+}=R p^{(n)}(1+n) \\
\frac{d}{d s}\left(r N_{-}\right)+(1-n) N_{-}=R p^{(n)}(1-n) \\
N_{\theta}^{(n)}=R p^{(n)}-N_{s}^{(n)}
\end{gathered}
$$

where

$$
\begin{aligned}
& N_{+}=N_{g}^{(n)}+N_{a \theta}^{(n)} \\
& N_{-}=N_{a}^{(n)}-N_{\rho \theta}^{(n)}
\end{aligned}
$$


(see Flügge [1973], p. 43). The solutions for $N_{+}$and $N_{-}$are then given by

$$
N_{ \pm}=R(1 \pm n) r^{-2 \mp n} \int p^{(n)} r^{1 \pm n} d r
$$

The membrane strains can be determined directly from the constitutive relations $(6, A .1-3)$, and the displacements, from the kinematic relations (18), are given by

$$
\begin{aligned}
& u_{r}^{(n)}=\int_{0}^{r} \varepsilon_{\theta}^{(n)} d s \\
& u_{z}^{(n)}=\int_{0}^{r} \frac{r}{R} \varepsilon_{\theta}^{(n)} d s \\
& u_{\theta}^{(n)}=\left(r \varepsilon_{\theta}-u_{r}\right) / n
\end{aligned}
$$

For $n=1$, Eqs. (55) and (56) yield

$$
N_{\rho}^{(1)}=N_{\rho \theta}^{(1)}=R r^{-3} \int_{0}^{r} p^{(1)} r^{\prime 2} d r^{\prime}
$$

In the subsequent analysis, the solution (58) is used to represent the membrane stresses due to an asymmetric pressure load.

\section{$\S 8$ Solution Procedure}

As in the previous investigation (Steele and Balch, 1987), the total solution is decomposed into particular and complementary solutions. For the nth harmonic,

$$
Y^{(n)}=Y_{p .}^{(n)}+Y_{c}^{(n)}
$$

where $Y_{p}^{(n)}$ is the particular solution which satisfies Eq. (24), but does not in general satisfy the boundary conditions at the edge $s=s_{e}$, and $Y_{c}^{(n)}$ is the complementary solution, which is the general solution to the homogeneous equation (31).

In the present work, the particular solution is taken to be zero for all $n \geq 2$. For $n=0$, the particular solution is taken to be the axisymmetric membrane solution for uniform pressure loading (see Steele and Balch, 1987). For $n=1$, the particular solution is taken to be the "wind load" solution (58), which is evaluated by assuming a piecewise linear radial pressure distribution, with pressure data from a set of sample points along the meridian of the paraboloid. The displacements are determined by numerical integration of Eqs. (57).

The complementary solution $Y_{c}^{(n)}$ can be expressed

$$
\begin{aligned}
Y_{c}^{(n)}= & C_{1}^{(n)} Y_{\mathrm{SH}}^{(n)}+C_{2}^{(n)} Y_{\mathrm{EB} 1}^{(n)}+C_{3}^{(n)} Y_{\mathrm{EB2}}^{(n)} \\
& +C_{4}^{(n)} Y_{\mathrm{ME}}^{(n)}+C_{3}^{(n)} Y_{\mathrm{IN}}^{(n)}
\end{aligned}
$$


where $Y_{S H}^{(n)}$ is the asymptotic solution for the local transverse shear edge effect; $Y_{\mathrm{EB} 1}^{(n)}$ and $Y_{\mathrm{EB2}}^{(n)}$ are the asymptotic solutions for the rapidly decaying bending edge effects; $Y_{\mathrm{ME}}^{(\boldsymbol{n})}$ and $\mathbf{Y}_{\mathrm{IN}}^{(\boldsymbol{n})}$ are the membrane and inextensional shallow shell solutions; and the $C_{i}^{(n)}$ are unknown constants.

The five unknown constants $C_{i}^{(n)}$ are determined by enforcing five boundary conditions along the edge $s=s_{\mathrm{e}}$. The conditions are

$$
\begin{array}{llll}
\chi_{\theta}^{(n)}=\chi_{0}^{*} & \text { or } & M_{\theta}^{(n)}=M_{\theta}^{*} \\
\chi_{\theta}^{(n)}=\chi_{\theta}^{*} & \text { or } & M_{\theta \theta}^{(n)}=M_{\theta \theta}^{*} \\
u_{r}^{(n)}=u_{r}^{*} & \text { or } & N_{r}^{(n)}=N_{r}^{*} \\
u_{z}^{(n)}=u_{z}^{*} & \text { or } & N_{z}^{(n)}=N_{z}^{*} \\
u_{\theta}^{(n)}=u_{\theta}^{*} & \text { or } & N_{\theta \theta}^{(n)}=N_{\theta \theta}^{*}
\end{array}
$$

where the superscript * denotes a prescribed value at the edge. Note that either a displacement quantity or its corresponding force quantity can be prescribed.

Equations (61) provide a linear algebraic system in the five unknowns $C_{i}^{(n)}$ for each harmonic. This system can be expressed

$$
[]^{(n)}\left[\begin{array}{l}
C_{1} \\
C_{2} \\
C_{3} \\
C_{4} \\
C_{3}
\end{array}\right]^{(n)}=[\mathrm{R}]^{(n)}
$$

where $\mathbf{M}$ and $\mathbf{R}$ are a known coefficient matrix and right-hand-side. The matrix $M$ is well conditioned when the boundary conditions are given as prescribed displacements. If all the boundary conditions are given as prescribed forces, however, conditioning problems may arise for very thin shells, due to the extremely low stiffnesses associated with the inextensional deformation modes.

\section{§9 Examples and Discussion}

The numerical results presented in this section were obtained from the DISH computer program, which is described in Appendix C. The program is a computerized implementation of the solutions and the solution procedure discussed in the preceding sections, and is capable of analyzing several harmonics of deformation in a few minutes or less on a PC. Examples are presented for a thin steel paraboloidal dish, with the geometry, material properties, and nominal pressure load given in Table 1. 
Table 1. Specifications for paraboloidal dish.

\begin{tabular}{|l|c|c|}
\hline \multicolumn{1}{|c|}{ Geometry } & Material Properties & Load \\
\hline$t=0.254 \mathrm{~mm}$ & $E=209 \mathrm{GPa}$ & $p=2000 \mathrm{~Pa}$ \\
$r_{\mathrm{e}}=7.5 \mathrm{~m}$ & $\nu=0.3$ & \\
$f=9 \mathrm{~m}$ & & \\
\hline
\end{tabular}

\subsection{Prescribed Radial Edge Deformation}

Consider a prescribed radial edge displacement of the form

$$
u_{r}=u_{r}^{(n)} \cos n \theta
$$

with

$$
u_{r}^{(n)}=1.0 \mathrm{~mm} \quad ; \quad \chi_{\theta}^{(n)}=\chi_{\theta}^{(n)}=u_{z}^{(n)}=u_{\theta}^{(n)}=0
$$

at the edge. This can be envisioned as a $\cos n \theta$ radial perturbation of a nominal axisymmetric configuration in which the edge of the paraboloid is clamped. Note that the paraboloid is also subjected to the pressure load $p=2000 \mathrm{~Pa}$.

The displacements $u_{r}$ and $u_{z}$ are plotted in Fig. 6 for the case $n=2$. For comparision, the displacements for the nominal axisymmetric case of a clamped edge is shown (solid lines in Fig. 6). For $\theta=0^{\circ}$-(dashed lines), the radial displacement $u_{r}$ equals $1.0 \mathrm{~mm}$ at the outer edge, and the magnitude of the vertical displacement is less than for the axisymmetric case. But along the radial line $\theta=90^{\circ}$ (dotted lines), the magnitude of the vertical displacement is greater than for the axisymmetric case. This is the expected behavior of an $n=2$ deformation mode.

The rotations $\chi_{0}$ and $\chi_{\theta}$ for the case $n=2$ are plotted in Fig. 7. For $\theta=0^{\circ}$ (dashed line) the peak meridional rotation $\chi_{\theta}$ is smaller than for the nominal axisymmetric case (solid line), but for $\theta=90^{\circ}$ (dotted line), the magnitude of the peak meridional rotation $\chi_{0}$ is greater than for the axisymmetric case. Note that the maximum circumferential rotations $\chi_{\theta}$, which occur along the radial line $\theta=45^{\circ}$ (dot-dashed line in Fig. 7), are much smaller than the maximum meridional rotations.

Because zero meridional rotation is prescribed at the edge, the values

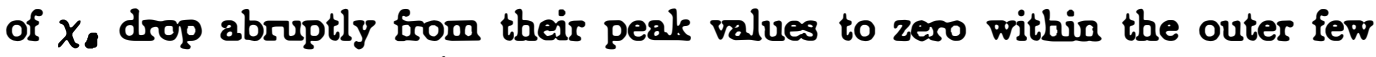
millimeters of the edge (see Fig. 7). This indicates a rapidly decaying edge bending effect. A much more slowly decaying edge bending effect with a decay distance $\delta \sim 1 \mathrm{~m}$ is also evident.

Figure 8 shows the stress resultants $N$, and $N_{\theta}$ for the case $n=2$. The values of the stress resultants for the nominal axisymmetric case are shown for comparison (solid line). Along $\theta=0^{\circ}$, the meridional stress $N$, associated with the $n=2$ edge deformation is uniformly greater than for the axisymmetric case, and the ciroumferential stress $N_{\theta}$ exhibits a relatively smaller drop near the edge (dashed lines). But along $\theta=90^{\circ}$ (dotted lines), 


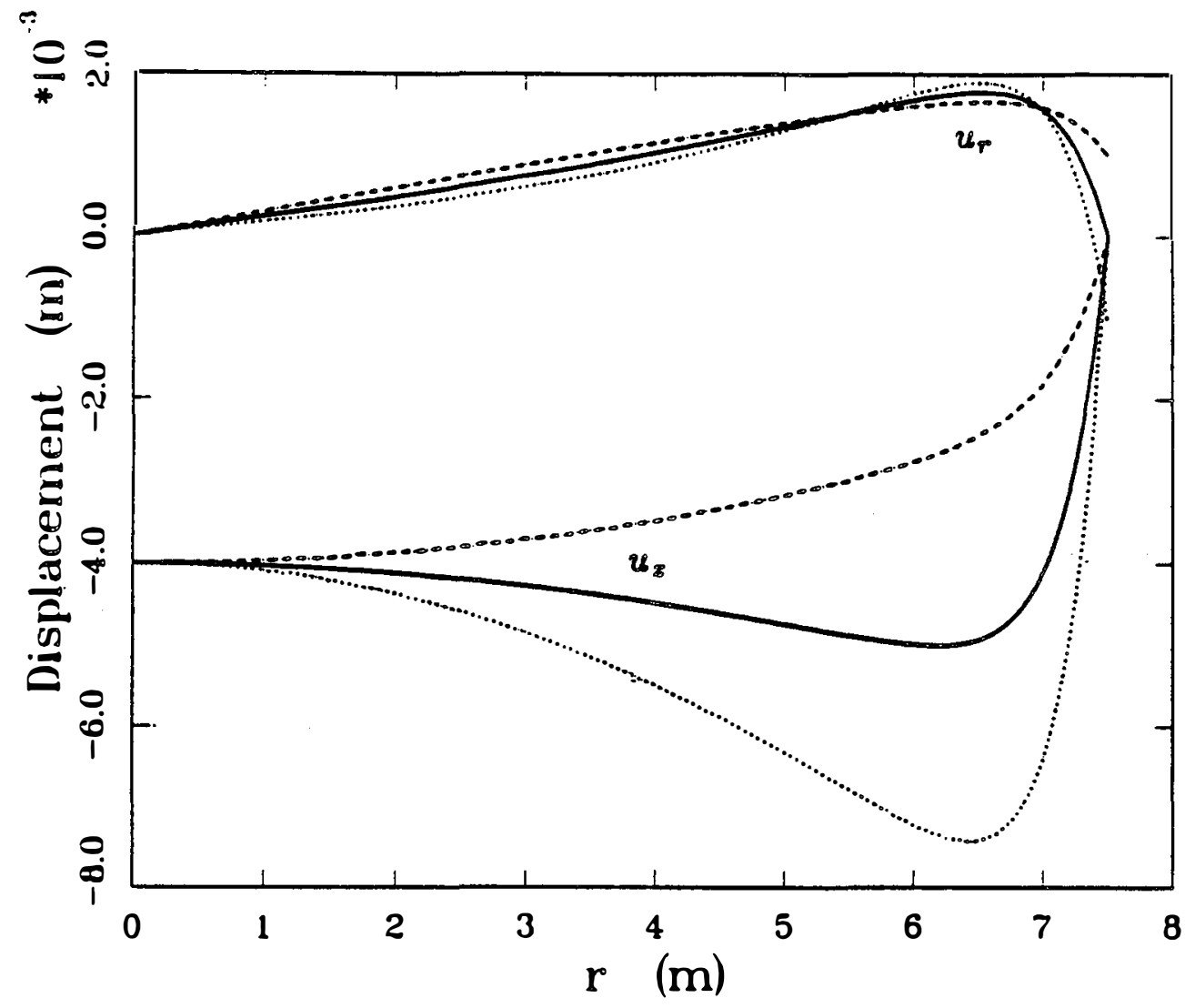

Figure 6. Radial displacement $u_{r}$ and vertical displacement $u_{z}$ as functions of $r$ for a paraboloidal shell subjected to a $\cos 2 \theta$ prescribed radial edge displacement. Results shown for $\theta=0^{\circ}$ (dashed lines) and $\theta=90^{\circ}$ (dotted lines). The results for the axisymmetric case of a clamped edge are shown for comparison (solid lines). 


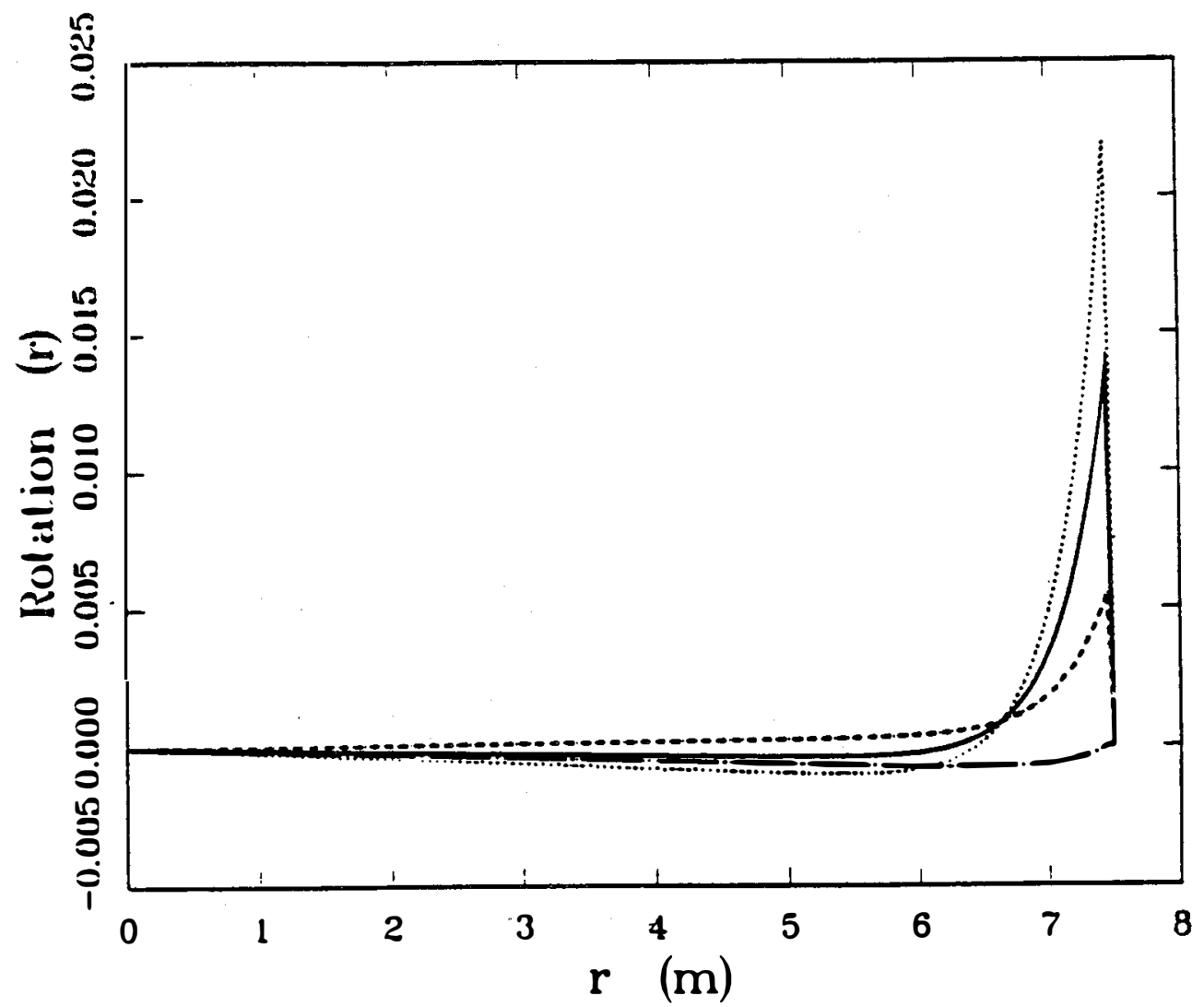

Figure 7. Rotations $\chi_{0}$ and $\chi_{\theta}$ as functions of $r$ for a paraboloidal shell subjected to a $\cos 2 \theta$ prescribed radial edge displacement. The meridional rotation $\chi_{0}$ is shown for $\theta=0^{\circ}$ (dashed line) and $\theta=90^{\circ}$ (dotted line), and for the axisymmetric case of a clamped edge for comparison (solid line). The cinumferential rotation $\chi_{\theta}$ is shown for $\theta=45^{\circ}$ (dot-dashed line). Note the large meridional rotations near the edge, and the abrubt drop to zero due to the clamped edge condition. 


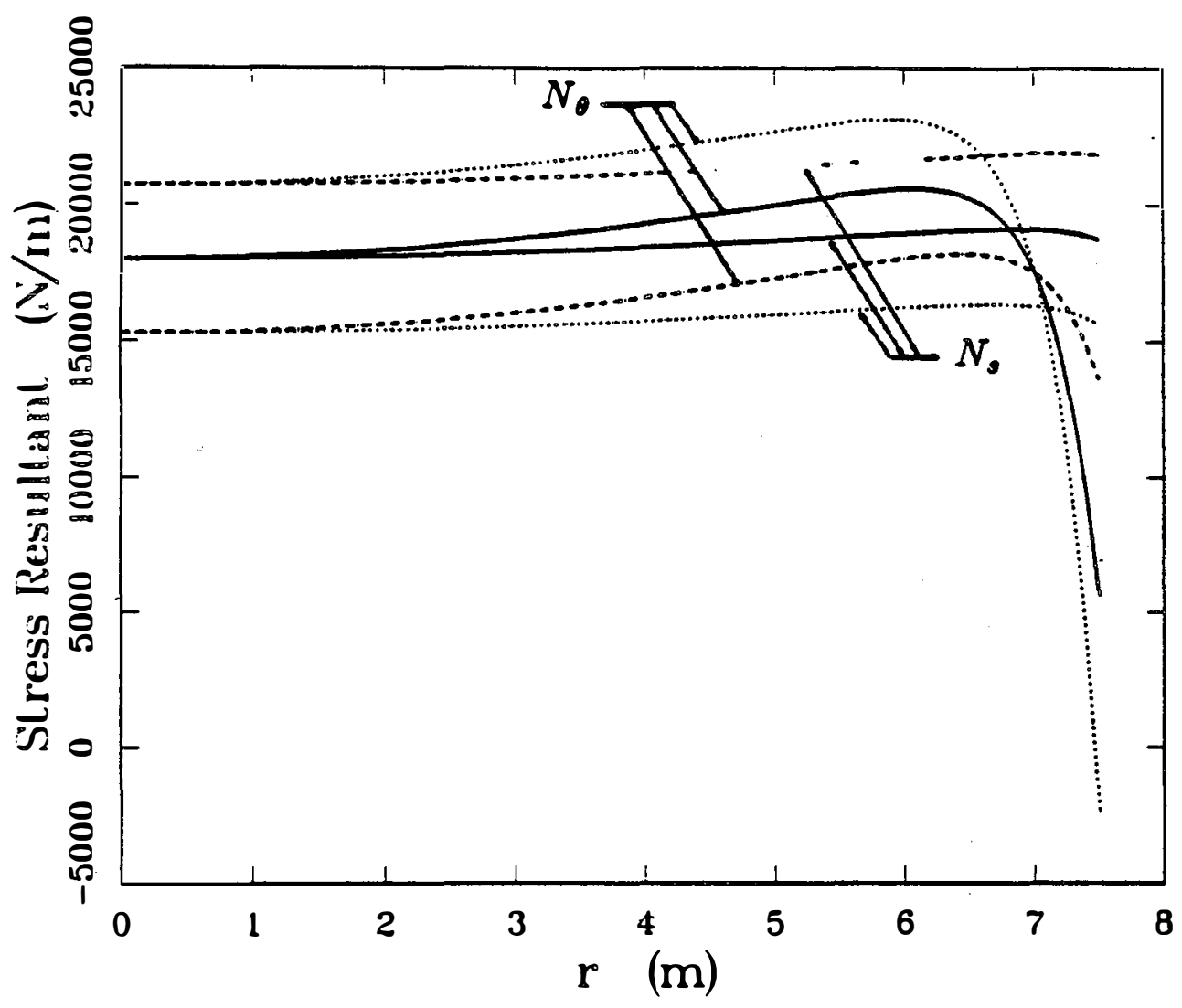

Figure 8. Stress resultants $N$, and $N_{\theta}$ as functions of $r$ for a paraboloidal shell subjected to a $\cos 2 \theta$ prescribed radial edge displacement. The stress resultants are shown for $\theta=$ $0^{\circ}$ (dashed lines) and $\theta=90^{\circ}$ (dotted lines), and for the axisymmetric case of a clamped edge for comparison (solid lines). Note the significant drop in the circumferential stress $N_{0}$ near the edge for $\theta=90^{\circ}$. 
the meridional stress is uniformly less than for the axisymmetric case, and the drop in circumferential stress near the edge is quite pronounced.

Note that the effect of the $n=2$ edge deformation on the meridional stress $N_{3}$ is not local to the edge zone, but is distributed uniformly over the shell. This is due to the participation of the $n=2$ membrane solution, which can be characterized approximately as a state of uniform tension along $\theta=0^{\circ}$ with uniform compression along $\theta=90^{\circ}$.

The radial and vertical displacements $u_{r}$ and $u_{z}$ are shown in Fig. 9 for higher barmonics of prescribed radial edge displacement, corresponding to $n=4$ (dashed lines), $n=16$ (dotted lines), and $n=64$ (dot-dashed lines). Again, the displacements for the nominal axisymmetric case of a clamped edge are shown for comparison (solid lines). It is evident that for higher harmonics of edge displacement, the edge effects decay more rapidly, becoming more localized to the edge zone.

For a given amplitude $u_{r}^{(n)}$ of edge displacement, the stresses grow with $n$ (not shown). This reflects the greater stiffness of the shell for higher harmonics of edge deformation. Because the present analysis is based on the linearized moderate rotation terms with no nonlinear update of the membrane stresses, the accuracy will therefore deteriorate as $n$ is increased while $u_{r}^{(n)}$ is held fixed.

\subsection{Prescribed Vertical Edge Deformation}

Now consider the case of a prescribed vertical edge displacement of the form

$$
u_{z}=u_{z}^{(n)} \cos n \theta
$$

with

$$
u_{z}^{(n)}=1.0 \mathrm{~mm} \quad ; \quad \chi_{\theta}^{(n)}=\chi_{\theta}^{(n)}=u_{r}^{(n)}=u_{\theta}^{(n)}=0
$$

at the edge.

Figure 10 shows the radial and vertical displacements $u_{r}$ and $u_{z}$ for the case $n=2$ and Fig. 11 shows the meridional and vertical stress resultants $N_{s}$ and $N_{\theta}$. Figure 12 shows the displacements $u_{r}$ and $u_{z}$ for $n=4,16$, and 64. For brevity, detailed commentary will not be included outside of the captions, since the results are qualitatively similar to those of the previous example in which the radial edge displacement was prescribed.

\subsection{Asymmetric Pressure Loading}

For the final example, we consider a "wind load"

$$
p_{n}=p_{w} \sin \phi \cos \theta
$$

where $p_{n}$ is the normal pressure on the shell, $p_{w}$ is a known wind pressure, and $\phi$ is defined in Fig. 3. The form (67) is borrowed from Flügge (1973). 


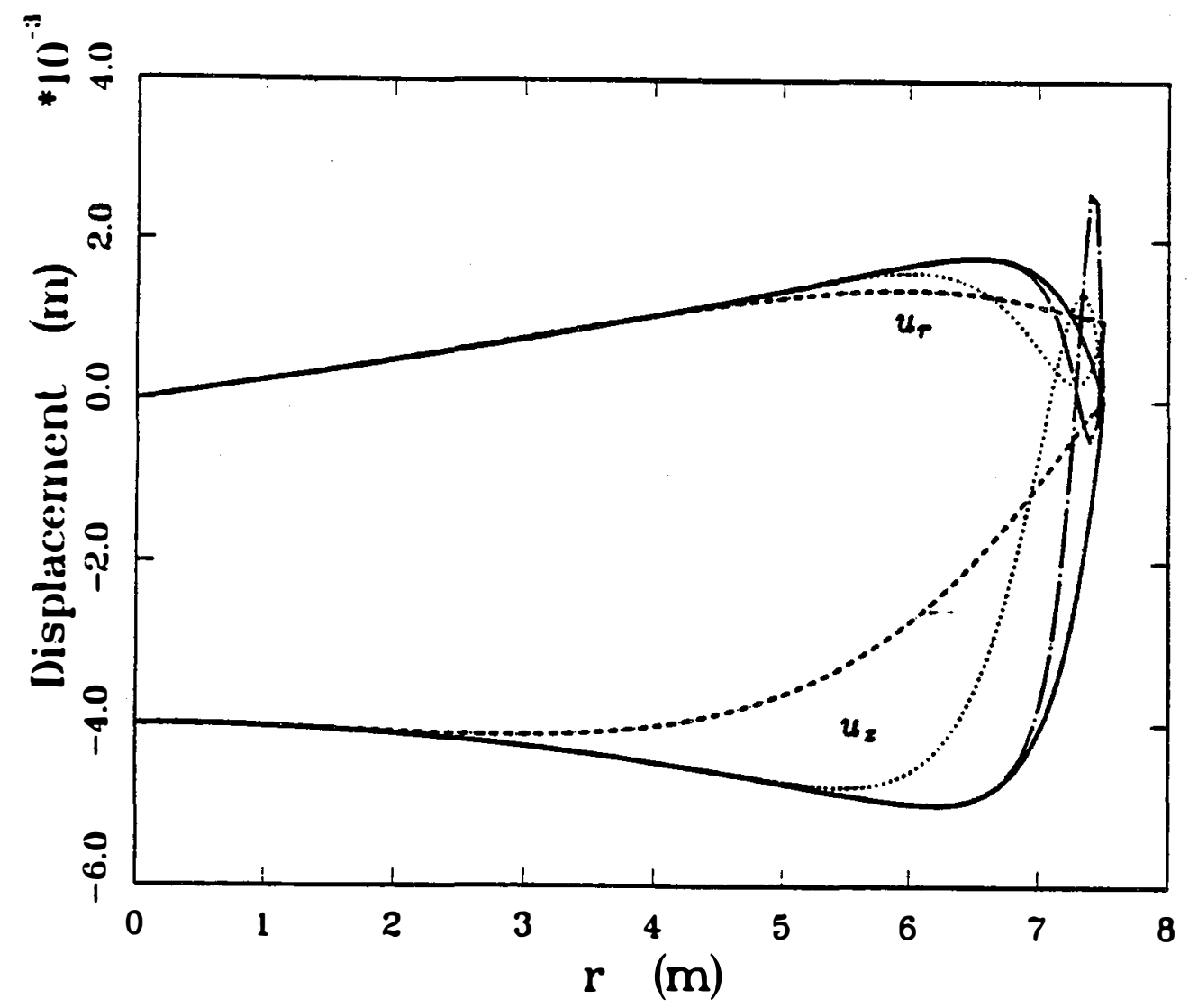

Figure 9. Radial displacement $u_{r}$ and vertical displacement $u_{z}$ along the line $\theta=0^{\circ}$ for a paraboloidal shell subjected to $\cos n \theta$ prescribed radial edge displacements. Results shown for $n=4$ (dashed lines), $n=16$ (dotted lines), and $n=64$ (dot-dashed lines). The results for the axisymmetric case of a clamped edge are shown for comparison (solid lines). With incressing $n$ the edge effects decay more rapidly; outside the edge zone, the displacements approach those of the axisymmetric case. 


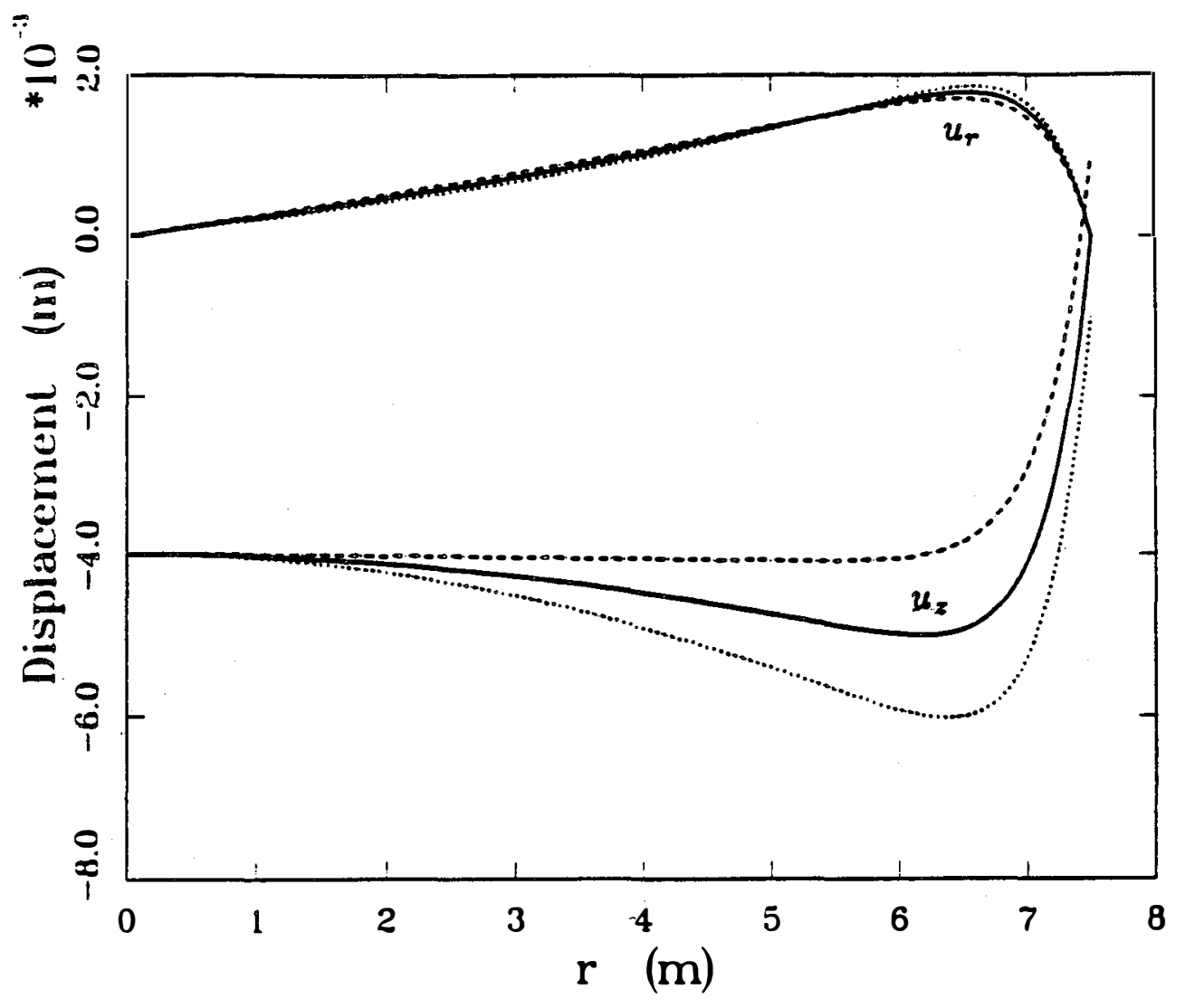

Figure 10. Radial displacement $u_{r}$ and vertical displacement $u_{z}$ as functions of $r$ for a paraboloidal shell subjected to a $\cos 2 \theta$ prescribed vertical edge displacement. Results shown for $\theta=0^{\circ}$ (dashed lines) and $\theta=90^{\circ}$ (dotted lines). The results for the axisymmetric case of a clamped edge are shown for comparison (solid lines). 


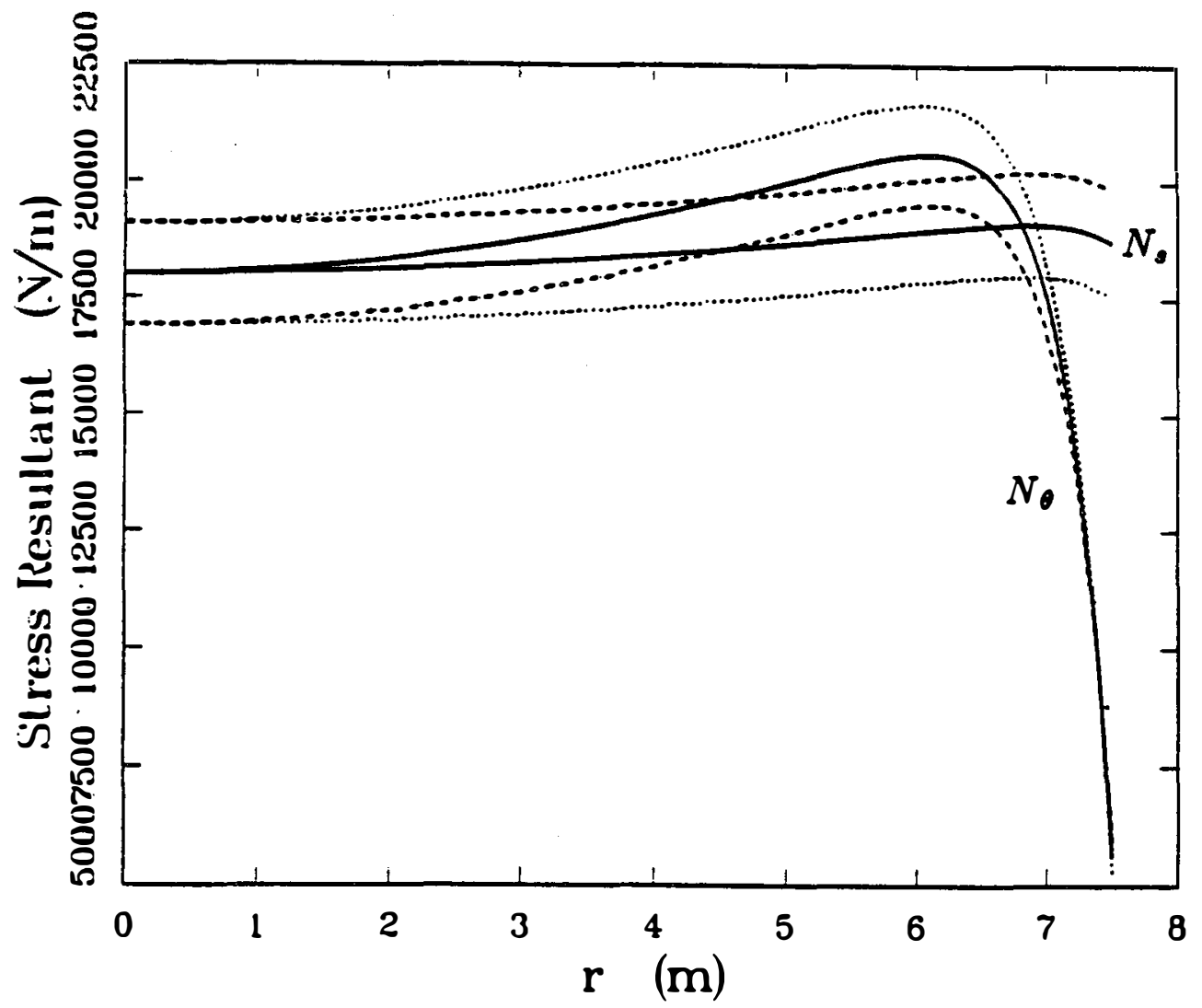

Figure 11. Stress resultants $N_{\text {, }}$ and $N_{\theta}$ as functions of $r$ for a paraboloidal shell subjected to a $\cos 2 \theta$ prescribed vertical edge displacement. The stress resultants are shown for $\theta=$ $0^{\circ}$ (dashed lines) and $\theta=90^{\circ}$ (dotted lines), and for the axisymmetric case of a clamped edge for comparison (solid lines). Note the significant drop in the circumferential stress $N_{0}$ near the edge. 


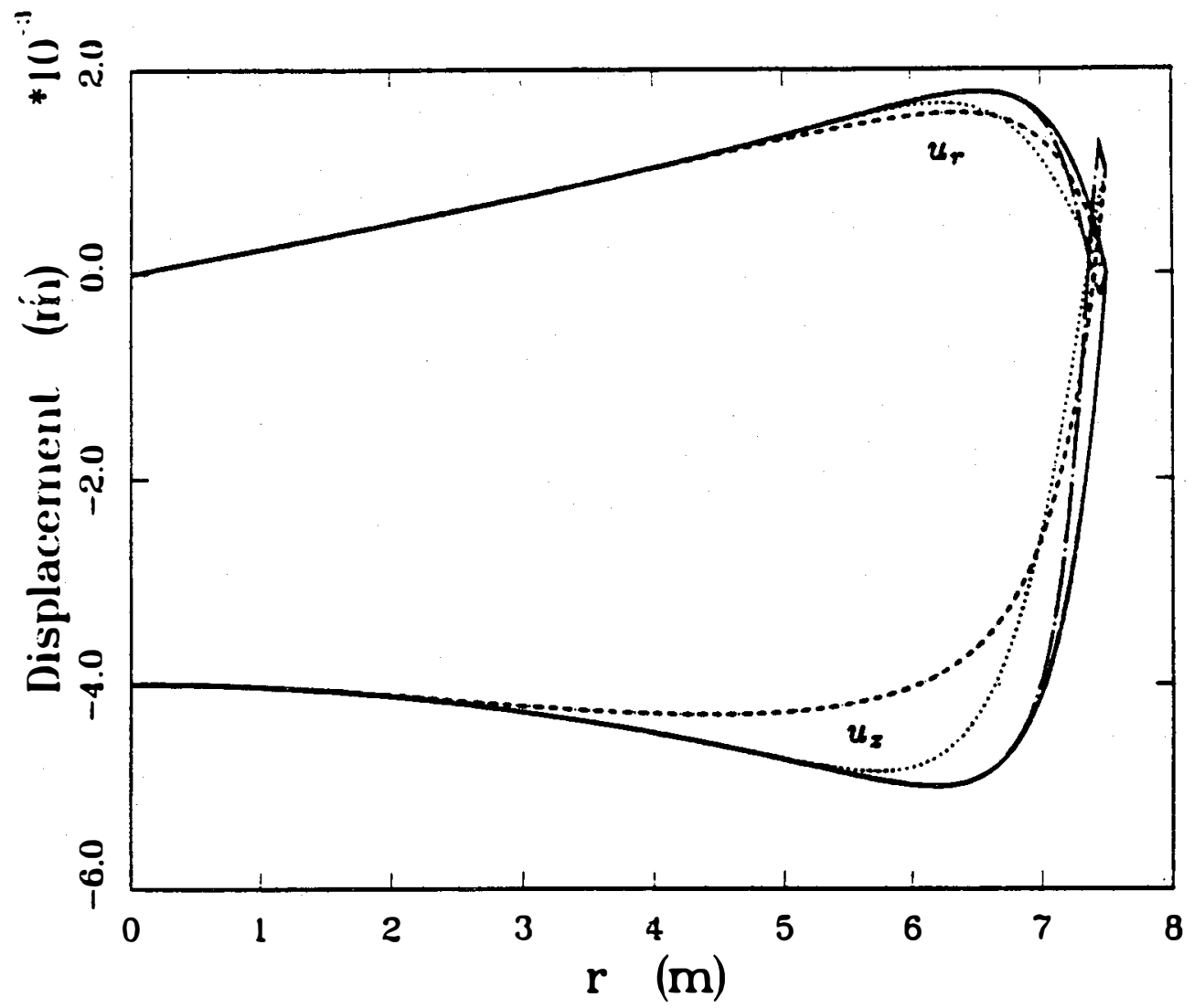

Figure 12. Radial displacement $u_{r}$ and vertical displacement $u_{x}$ along the line $\theta=0^{\circ}$ for a paraboloidal shell subjected to $\cos n \theta$ prescribed vertical edge displacements. Results shown for $n=4$ (dashed lines), $n=16$ (dotted lines), and $n=64$ (dot-dashed lines). The results for the axisymmetric case of a clamped edge are shown for comparison (solid lines). With increasing $n$ the edge effects decay more rapidly; outside the edge zone, the displacements approach those of the axisymmetric case. 
The edge of the paraboloid is taken as clamped (all displacements and rotations are prescribed to be zero), and the nominal pressure is $2000 \mathrm{~Pa}$.

Figure 13 shows the meridional stress resultant $N_{1}$ (solid line) and the circumferential stress resultant $N_{\theta}$ (dashed line) evaluated along $\theta=0^{\circ}$ for the case $p_{w}=1000 \mathrm{~Pa}$. The marks in Fig. 13 were computed using the membrane results for the spherical shell from Flügge (1973), p. 44. Good agreement is observed except near the edge, where edge bending effects are siguificant due to the clamped edge conditions. Note that much of the load is carried by the circumferential "hoop" stress.

Figure 14 shows the radial and vertical displacements $u_{r}$ and $u_{z}$ along the radial line $\theta=0^{\circ}$, for $p_{w}=0,1000$, and $2000 \mathrm{~Pa}$, and Fig. 15. shows the meridional rotation $\chi_{s}$. As $p_{w}$ is increased, the rotation $\chi_{s}$ near the edge at $\theta=0^{\circ}$ also increases, although the qualitative behavior of the edge effects along $\theta=0^{\circ}$ remains the same (see Fig. 15).

\section{$\S 10$ Conclusions}

A tenth-order matrix formulation was presented for non-axisymmetric deformations of shells of revolution. The formulation includes the moderate rotation terms which become important in the presence of high initial prestress. Based on this formulation, asymptotic solutions were obtained for non-axisymmetric edge bending effects in a pressure-loaded paraboloidal shell. For the membrane and inextensional responses to edge loads, solutions were derived using shallow shell theory. Membrane solutions for asymmetric pressure loads were also derived.

The various solutions for the paraboloidal shell were incorporated into a computer program which can be run in a few minutes on a PC. A thin steel parabolic dish was analyzed, and examples were presented which demonstrate the effect of prescribing circumferential harmonics of edge displacement, and the effect of an asymmetric pressure load. For low harmonics of deformation, the decay distances of the bending edge effects were found to be comparable to those for the axisymmetric case. This is due to the extremely large radius-to-thickness ratio of the shell which was analyzed.

For problems involving prescribed edge displacements, membrane solutions contribute significantly. In particular, the membrane stresses due to an $n=2$ edge deformation do not decay appreciably with increasing distance from the edge. For asymmetric pressure loading, the results of the present analysis agree with membrane theory, except near the edge, where edge bending effects become important. 


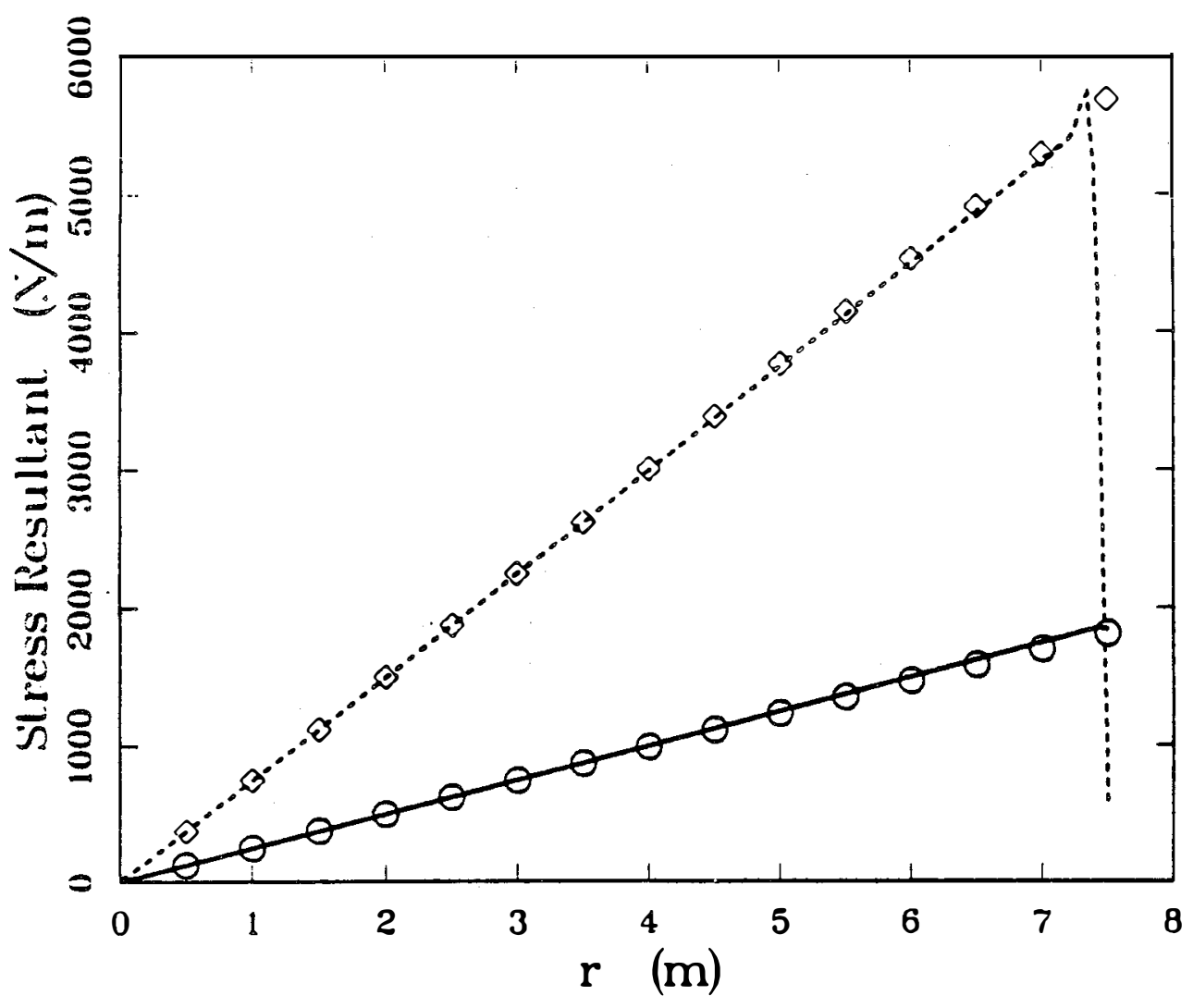

Figure 13. Stress resultants $N_{s}$ (solid line) and $N_{\theta}$ (dashed line) along $\theta=0^{\circ}$ for a clamped paraboloidal shell subjected to the asymmetric load $p_{n}=p_{w} \sin \phi \cos \theta$, with $p_{w}=1000 \mathrm{~Pa}$ and a nominal pressure load of $2000 \mathrm{~Pa}$. The circles and diamonds are the membrane solution from Flügge (1973), which agrees with the present analysis except near the edge. The present analysis includes the rapidly decaying edge bending effects, which account for the abrupt drop in the circumferential stress $N_{\theta}$ near the edge. 


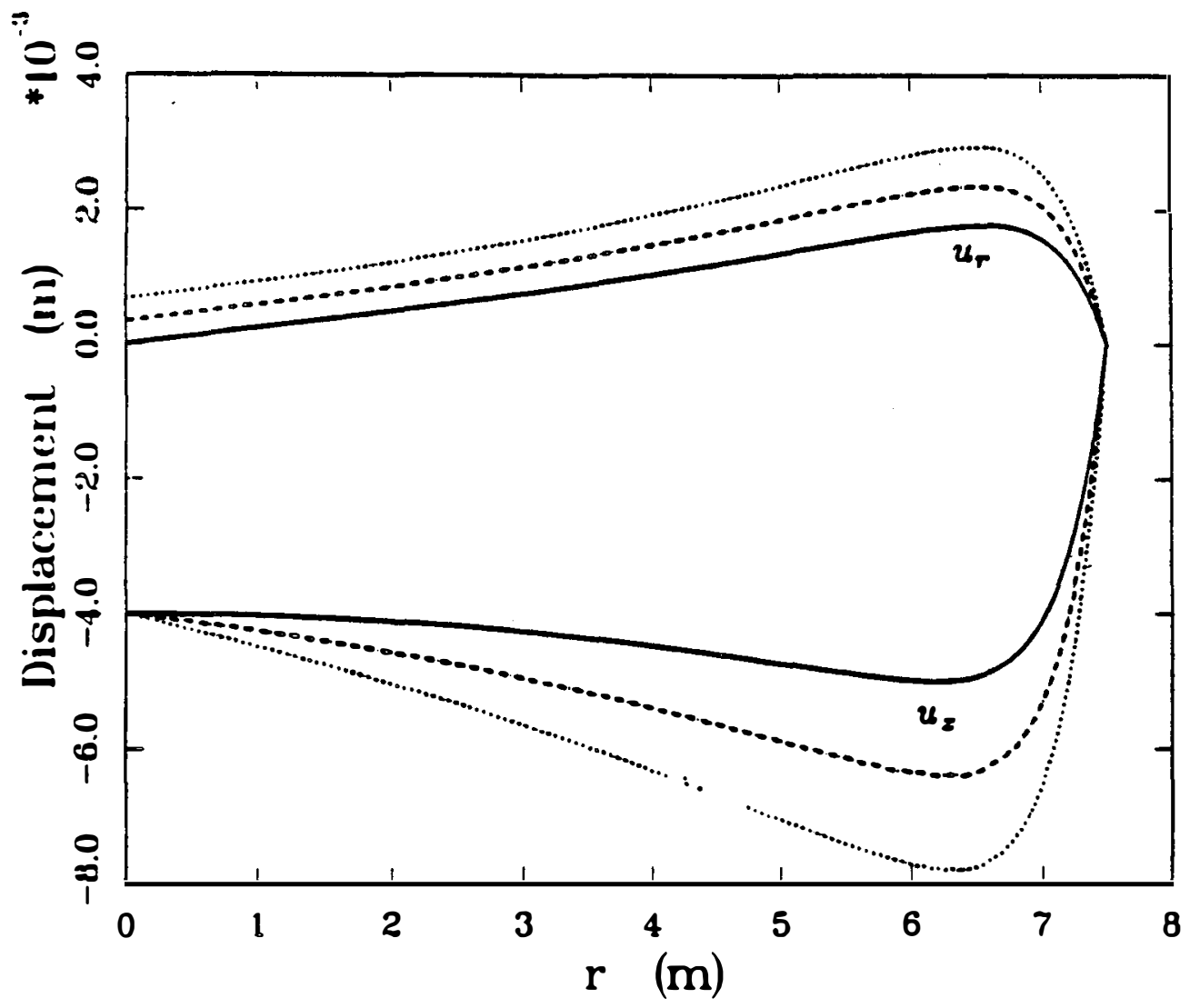

Figure 14. Displacements $u_{r}$ and $u_{z}$ along $\theta=0^{\circ}$ for a paraboloidal shell with a clamped edge subjected to the asymmetric load $p_{n}=p_{w} \sin \phi \cos \theta$, for $p_{w}=1000 \mathrm{~Pa}$ (dashed lines) and $p_{w}=2000 \mathrm{~Pa}$ (dotted lines). The displacements of the axisymmetric solution with the nominal pressure load of $2000 \mathrm{~Pa}$ are shown for comparison (solid lines). 


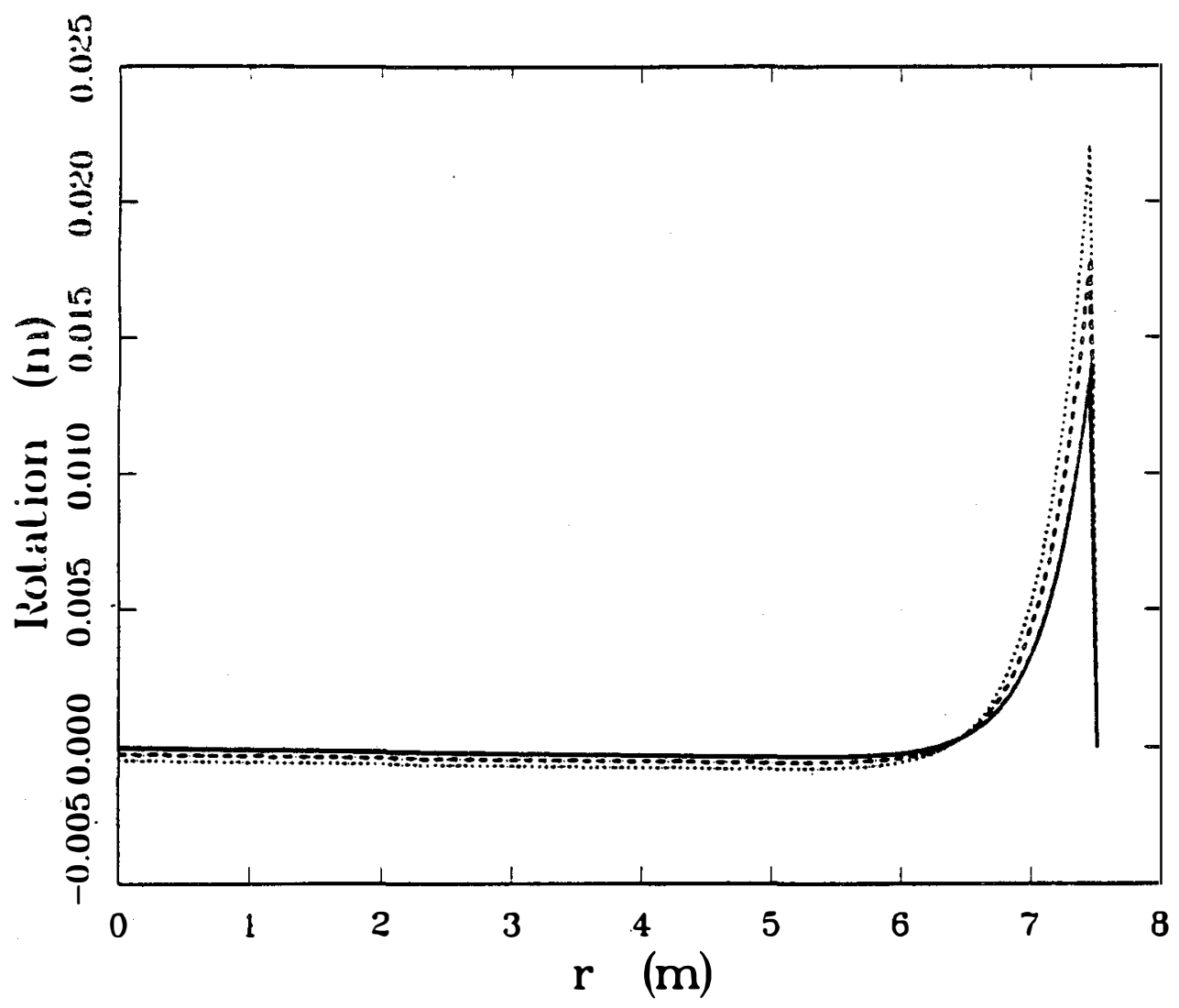

Figure 15. Meridional rotation $\chi_{\text {, along }} \theta=0^{\circ}$ for a paraboloidal shell with a clamped edge subjected to the asymmetric load $p_{n}=p_{w} \sin \phi \cos \theta$, for $p_{w}=1000 \mathrm{~Pa}$ (dashed line) and $p_{w}=2000 \mathrm{~Pa}$ (dotted line). The rotation $\chi_{s}$ of the axisymmetric solution with the nominal pressure load of $2000 \mathrm{~Pa}$ is shown for comparison (solid line). 


\section{References}

Flügge, W. (1973). Stresses in Shells, Second Edition. (Springer-Verlag, New York.)

Kutscher, C., Lewandowski, A., Wood, R., Wendelin, T., and Jorgensen, G. (1988). Membrane Dish Research Task FY 1987 Progress Report. Solar Energy Research Institute, Golden, $\mathrm{CO}$.

MACSYMA (1983). MACSYMA Reference Marual, Version Ten, Second Printing. (The Mathlab Group, Laboratory for Computer Science, MIT, and Symbolics, Inc., Cambridge.)

Ranjan, G. V. and Steele, C. R. (1980). "Nonlinear corrections for edge bending of shells," J. Appl. Mech. 47, 861-65.

Reissner, E. (1950). "On axisymmetrical deformations of thin shells of revolution," in Proceedings of Symposia in Applied Mathematics 3, 2752.

Steele, C. R. (1980). "Asymptotic solutions without special functions for steep and shallow shells," Mechanics Today 5, 483-94.

Steele, C. R. (1988). Shells of Revolution with Axisymmetric Loading. Stanford University course notes.

Steele, C. R. and Balch, C. D. (1987). "Edge Effects in a Shell of Revolution under Pressure Load," Shelltech Report 87-1.

Whassow, W. S. (1958). Allgemeine Schalentheorie und ihre Anwendung in der Technik. (Alademie-Verlag, Berlin.)

\section{Appendix A: Constitutive Matrices for Isotropic Behavior}

For isotropic behavior, the constitutive matrices $\Gamma_{11}, \Gamma_{12}, \Gamma_{21}$, and $\Gamma_{22}$ in Eq. (6) are given by

$$
\begin{aligned}
& \Gamma_{11}=E t\left[\begin{array}{ccccc}
c^{2} & 0 & 0 & 0 & 0 \\
0 & \frac{c^{2}(1-\nu)}{2} & 0 & 0 & 0 \\
0 & 0 & \frac{1}{\mu} & 0 & 0 \\
0 & 0 & 0 & \frac{1}{1-\nu^{2}} & 0 \\
0 & 0 & 0 & 0 & \frac{1}{2(1+\nu)}
\end{array}\right] \\
& \Gamma_{21}=\Gamma_{12}^{T}=E t\left[\begin{array}{ccccc}
\nu c^{2} & 0 & 0 & 0 & 0 \\
0 & 0 & 0 & 0 & 0 \\
0 & 0 & 0 & \frac{\nu}{1-\nu^{2}} & 0
\end{array}\right]
\end{aligned}
$$




$$
\Gamma_{22}=E t\left[\begin{array}{ccc}
c^{2} & 0 & 0 \\
0 & \frac{1}{\mu} & 0 \\
0 & 0 & \frac{1}{1-\nu^{2}}
\end{array}\right]
$$

where $E$ is the elastic modulus, $\nu$ is Poisson's ratio, $t$ is the shell thickness, and $c$ is given by Eq. (38)

\section{Appendix B: Coefficient Matrices for Isotropic Behavior}

The coefficient matrices $E^{(n)}, C$, and $K^{(n)}$ in Eqs. (22) and (24) can be calculated from Eqs. (23). For isotropic behavior, the explicit forms of the matrices are

$$
\begin{gathered}
\mathbf{E}^{(n)}=\frac{1}{r}\left[\begin{array}{ccccc}
\nu \cos \phi & \mp n & r \sin \phi & -r \cos \phi & 0 \\
\pm \nu n & -\cos \phi & 0 & 0 & 0 \\
0 & 0 & \nu \cos \phi & \nu \sin \phi & \mp n \cos \phi \\
0 & 0 & 0 & 0 & \mp n \sin \phi \\
0 & 0 & \pm \nu n \cos \phi & \pm \nu n \sin \phi & -\cos \phi
\end{array}\right] \quad(B .1) \\
\mathbf{C}=\frac{1}{E t r}\left[\begin{array}{ccccc}
\frac{1}{c^{2}} \\
0 & \frac{2}{c^{2}(1-\nu)} & 0 & 0 & 0 \\
0 & 0 & \mu \sin ^{2} \phi+\left(1-\nu^{2}\right) \cos ^{2} \phi & \left(1-\nu^{2}-\mu\right) \sin \phi \cos \phi & 0 \\
0 & 0 & \left(1-\nu^{2}-\mu\right) \sin \phi \cos ^{2} & \mu \cos ^{2} \phi+\left(1-\nu^{2}\right) \sin ^{2} \phi & 0 \\
0 & 0 & 0 & 0 & 2(1+\nu)
\end{array}\right]
\end{gathered}
$$

$\mathbf{K}^{(\boldsymbol{n})}=$

$$
\frac{E t}{r}\left[\begin{array}{ccccc}
\frac{t^{2} \cos ^{2} \phi}{12} & \pm \frac{n t^{2} \cos \phi}{12} & 0 & 0 & 0 \\
\cdot & \frac{n^{2} t^{2}}{12}+\frac{r^{2}}{\mu} & \mp \frac{n r \sin \phi}{\mu} & \pm \frac{n r \cos \phi}{\mu} & -\frac{r \sin \phi}{\mu} \\
\cdot & \cdot & \frac{n^{2} \sin ^{2} \phi}{\mu}+1 & -\frac{n^{2} \sin \phi \cos \phi}{\mu} & \pm n\left(\frac{\sin ^{2} \phi}{\mu}+1\right) \\
\cdot & {[\text { Symmetric] }} & \cdot & \frac{n^{2} \cos ^{2} \phi}{\mu} & \mp \frac{n \sin \phi \cos \phi}{\mu} \\
\cdot & \cdot & . & \cdot & \frac{\sin ^{2} \phi}{\mu}+n^{2}
\end{array}\right]
$$

where the upper sign of $\{ \pm, \mp\}$ corresponds to $\cos n \theta$ circumferential dependence, and the lower sign corresponds to $\sin n \theta$ circumferential dependence. 


\section{Appendix C: Description of the DISH Computer Program}

This appendix provides a brief description of the DISH computer program for the analysis of paraboloidal shells. The program is written in standard FORTRAN-77 and can be run on an IBM PC. A sample input file is shown in the following table.

Table C-1. Sample input file for the DISH program.

Input File DISH.IN, for the DISH structural analysis program.

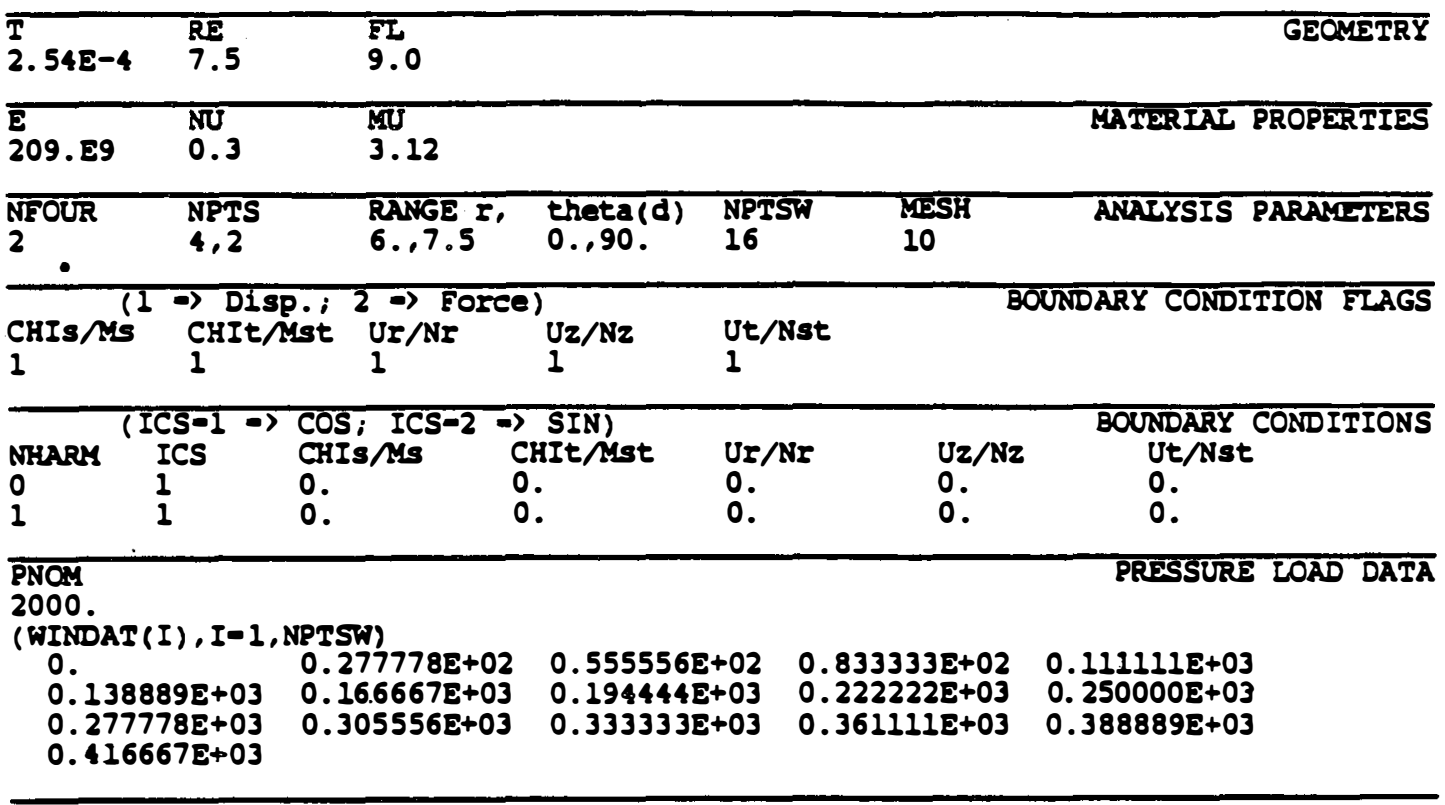

Table C-1 shows an input file for the analysis of a paraboloid with clamped edges and wind loading. The first line of numbers contains the thickness $T$, edge radius RE, and focal length FL. The second line of numbers contains the elastic modulus E, Poisson's ratio NU, and the shear flexibility factor MU (see Eq. [5]).

The parameters in the third line of numbers are

NFOUR: The total number of Fourier harmonics in the analysis, in this case $2(n=0$ and $n=1)$.

NPTS: Adjusts the density of the grid of output points. In this case, 4 points in the radial direction by 2 in the circumferential.

RANGE: The first two numbers are the minimum and maximum $r$ values of the output interval, in this case $6.0 \leq r \leq 7.5$. The second two numbers are the minimum and maximum $\theta$ values (in degrees) of the output interval, in this case $0 \leq \theta \leq 90$.

NPTSW: The number of pressure data points for an $n=1$ wind load analysis. Set to 0 if no wind load is included. 
MESH: A mesh parameter for the numerical integration which determines the displacements of the wind load solution. Any integer greater than 5 will yield adequate convergence in most cases.

The next line of input is the five integers under "Boundary Condition Flags." Each must be set to 1 or 2, where 1 indicates that a displacement or rotation is prescribed, and 2 indicates that the corresponding force or moment is prescribed. In the input file shown, the flags are set to 1 , which indicates that both rotations and all three displacements will be prescribed at the edge. To prescribe the meridional moment rather than the rotation, set the first flag to 2. Note that conditioning problems may arise if all the boundary conditions are chosen to be prescribed forces (see §8).

Under "Boundary Conditions" are one line of input for each harmonic in the analysis; in this case there are two lines. Each line includes

NHARM: The first integer in the line is the harmonic number; 0 for axisymmetric, 1 for $n=1$, etc.

ICS: The second integer should be set to 1 for a cosine harmonic, and 2 for a sine harmonic.

Boundary Conditions: The next five real numbers are the prescribed edge conditions for the harmonic specified at the beginning of the line. In this case, all are set to zero in order to get a clamped edge. To prescribe a radial displacement, set the number under " $\mathrm{Ur} / \mathrm{Nr}^{\prime}$ to the desired value, with the corresponding "boundary condition flag" set to 1 .

To specify the loading condition, assign pressure values to

PNOM: The nominal pressure load acting on the parabola, in this case 2000.

WINDAT: This is an array of pressure values from NPTSW equally spaced sample points which are distributed along the meridian $\theta=0$ from the center $r=0$ to the outer edge $r=r_{e}$. The circumferential variation is assumed to be $\cos \theta(n=1)$. The program will linearly interpolate the pressure between sample points. If no asymmetric pressure load is desired, set NPTSW to 0 and leave this array out.

When the program is run, it reads the input file DISH.IN, and generates the output file DISH.DAT, which is essentially an echo check of the input data. DISH.DAT includes some additional information, such as the dimensionless pressurization parameter RHO. High prestress is associated with a value of RHO which is large relative to unity. DISH.DAT also lists the roots of the characteristic equation (35), which are the decay constants of the asymptotic solutions. The DISH.DAT file generated by the input file in Table C-1 is listed in Table C-2.

The displacement and stress data is written to a second output file DISH.OUT. The DISH.OUT file generated by the input file in Table C-1 is listed in Table C-3. The current version of the DISH program records 13 quantities at each of the grid points specified in the input file. The 
13 quantities are the two rotations $\chi_{0}$ and $\chi_{0}$ of the normal, the three displacements $u_{r}, u_{z}$, and $u_{\theta}$, the three moment resultants $M_{s}, M_{\theta}$, and $M_{00}$, and the five stress resultants $N_{r}, N_{z}, N_{\theta}, N_{\theta}$, and $N_{0 \theta}$.

Table C-2. Sample output file DISH.DAT, from the DISH program.

Output File DISH.DAT, from the DISH structural analysis program.

Echo of Input Data:

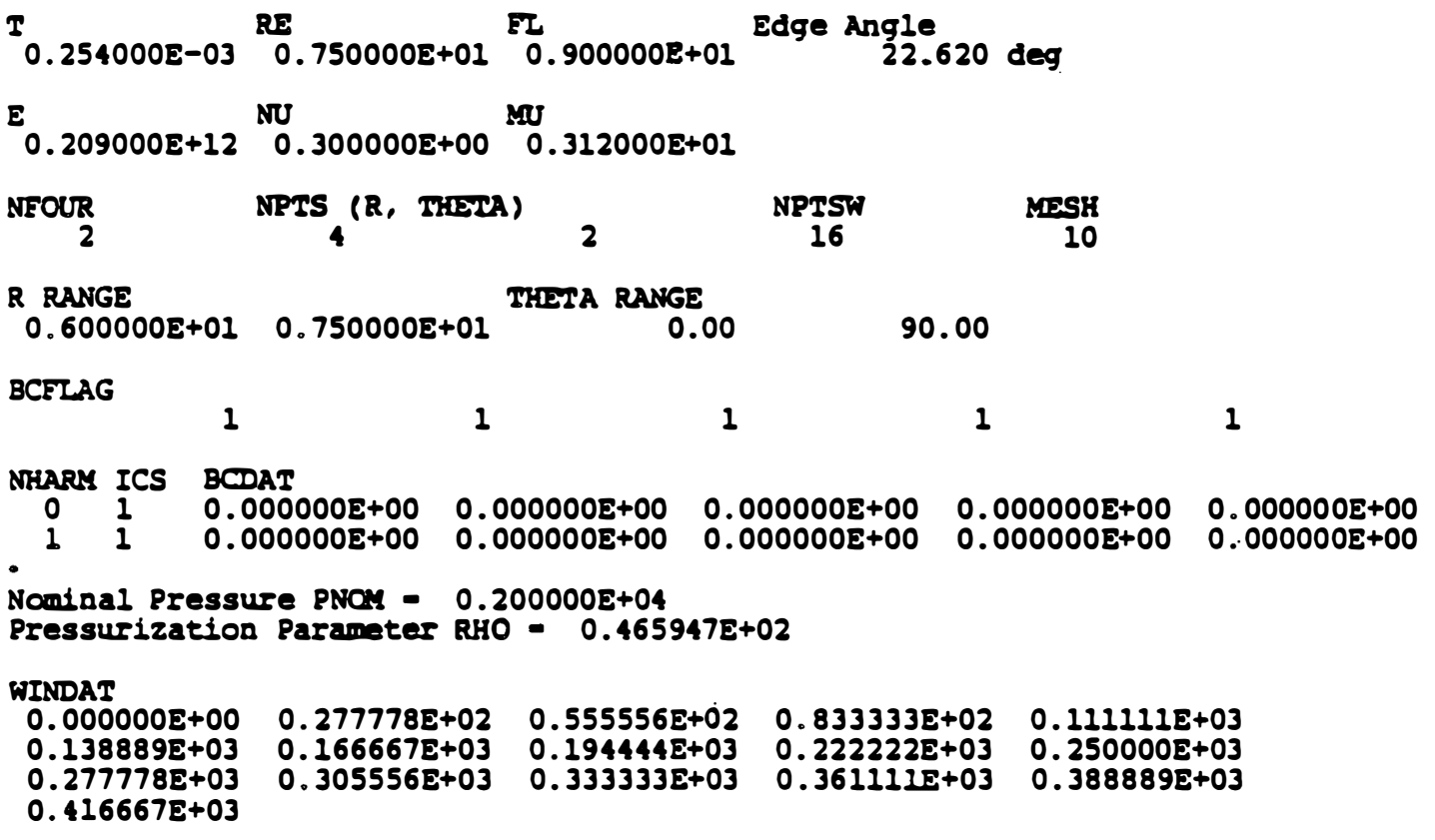

Decay Constants for $\mathrm{N}=0$

$(0.2493335+03,0.0000005+00)$

$(0.267569 E+01,0.000000 E+00)$

Decay Constants for $N$ - 1

$(0.124581 E+05,0.602718 E-24)$

$(0.249333 E+03,-0.281823 E-24)$

$(0.269064 E+01,-0.302915 E-24)$

$(0.123103 E+00,-0.576263 E-24)$

$(-0.105319 E-13,-0.113360 E-07)$ 
Table C-3. Sample output file DISH.OUT, from the DISH program.

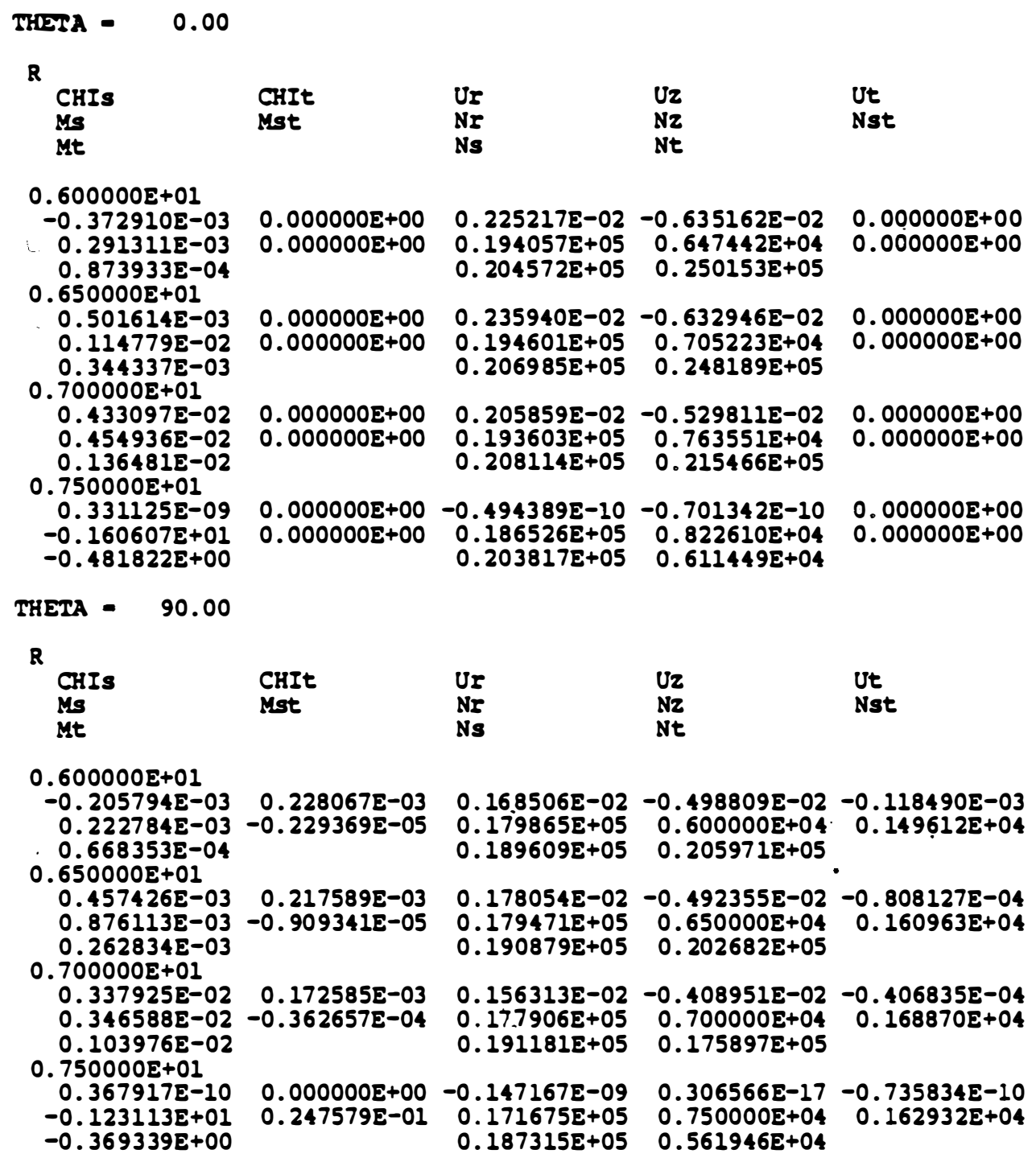




\title{
Procedures and Software for Dish Membrane Structural Analysis
}

\author{
by Chad D. Balch, Research Associate \\ Division of Applied Mechanics \\ Stanford University \\ Stanford, California 94305
}

Report to Membrane Dish Research Group, August 1988 


\subsection{Introduction}

This report summarizes work performed for SERI under Consultant Agreement No. CJ-8-00632-1, during the period June 20, 1988 through August 26, 1988. The primary purpose of this report is to document software for dish membrane structural analysis which was developed during the contract period. Topics to be discussed include the special purpose structural code DISH for the analysis of paraboloidal dish membranes, the analysis of ring/membrane interaction using DISH and the finite element code SUPERSAP, and coupled optical/structural analysis using DISH and the ray trace program OPTDSH. Example results are included in order to demonstrate the capability of the software, and to highlight some aspects of dish behavior.

\subsection{The DISH Program for Paraboloidal Membrane Structural Analysis}

The initial version of the DISH program for the non-axisymmetric analysis of thin paraboloidal shells was developed under Subcontract No. HX-8-18012-1 with Shelltech Associates. The program has been substantially restructured and enhanced during the present contract period in order to facilitate interfaces with other phases of the dish concentrator analysis.

The program relies on analytical solutions in the form of asymptotic series expansions. The governing shell equations and asymptotic solution methodology are discussed in detail by Steele and Balch (1987) and Balch and Steele (1988), and will not be elaborated on in the present report. It is reiterated here, however, that the theory places the following limitations on the applicability of the DISH program:

(i) thin shells;

(ii) small strains;

(iii) small displacement perturbations from the nominal membrane solution;

(iv) edge effects should die out with decay distances which are small relative to the radii of curvature, since the validity of the asymptotic solutions depends on this rapid decay;

(v) the edge angle should be less than about 30 degrees, since shallow shell solutions are employed for pure membrane and inextensional behavior.

The DISH program models membrane and bending behavior, and accounts for the geometric nonlinearity associated with the initial prestress due to pressure stabilization. Non-axisymmetric deformations are treated by a Fourier decomposition of 
displacements and forces in terms of circumferential harmonics. The response of the dish to general edge deformations can therefore be analyzed, usually in a matter of minutes or seconds on a PC.

A flowchart showing the input/output links between the DISH program and other processors is shown in Figure 1. Figure 1 provides an overview of the software to be discussed in this section.

\subsection{Compiling and Linking}

The FORTRAN source code for the DISH structural program is stored in two files: DISHI.FOR, which contains the main structural analysis routines; and DISH2.FOR, which contains canned routines for the eigensolver. Both files can be compiled with the Lahey F77L compiler. The code is essentially FORTRAN 77 standard, although a few minor modifications in DISHI.FOR may be necessary for other compilers. Once compiled, the object modules DISHI.OBJ and DISH2.OBJ should be linked to obtain the executable DISH.EXE.

\subsection{Preparation of Input}

The input to the DISH program consists of geometric parameters, material properties, boundary conditions, and loads, and is read from the input file DISH.IN. A sample input file, to be discussed in detail, is shown in Table 1. Typically, a copy of an old input file can be quickly edited to produce a desired new input file. The input read statements are unformatted, so that no special format is required within a given line of data. Note that some lines are merely label or spacer lines. DISH expects these lines to be there, and strips them off during input. The input file in Table 1 is now discussed item by item.

Run Title: The first line of input is taken to be the run title, and can be any ASCII string up to 80 characters long.

T, RE, FL: The thickness, radius, and focal length of the undeformed dish.

E, NU, MU: Young's modulus, Poisson's ratio, and the shear flexibility factor (see Shelltech Report 88-2). MU is 6/5 times the ratio of the in-plane elastic modulus to the transverse shear modulus, which is $12(1+N U) / 5$ for an isotropic material.

NFOUR: The number of Fourier harmonics to be included in the analysis; in this case, two: $n=0$ and $n=1$ (see Table 1 ).

NFOURT: The total number of Fourier harmonics listed in the input for the boundary conditions. If NFOUR $=$ NFOURT, then all harmonics which are listed are used. For NFOUR < NFOURT, only the first NFOUR harmonics are considered. This feature allows the program to ignore the boundary condition input for higher harmonics without requiring these lines to be deleted, since it may be necessary to include them later.

NPTSW: $\quad$ The number of pressure data points for an $n=1$ (asymmetric) wind load analysis, in this case, 16. Set to 0 if no wind load is included. 
MESH: $\quad$ The number of constant strain "elements" taken between pressure data points for the numerical computation of wind load displacements. The total number of elements along the meridian is therefore given by (NPTSW-1)*MESH, which should be about 100 or greater for a converged solution. The current limitation on the wind load data is that (NPTSW-1)*MESH+1 cannot exceed 301, i.e., a maximum of 300 constant strain elements along the meridian. Set to 0 if no wind load is included.

The next line of input contains the five integers under "Boundary Condition Flags." Each must be set to 1 or 2, where 1 indicates that a displacement (Ur, Uz, Ut) or rotation (CHIs, CHIt) is prescribed, and 2 indicates that the corresponding stress resultant (Nr, $\mathrm{Nz}$, Nst) or moment resultant (Ms, Mst) is prescribed. The directions of positive action for the force and displacement quantities are shown in Figure 2. Note that looking into the dish, positive theta is clockwise.

In the input file shown, the first flag is set to 2 which indicates that the meridional moment will be prescribed at the edge, and the next four flags are set to 1, which indicates that the circumferential rotation and all three displacements will be prescribed at the edge. Note that conditioning problems may arise if all the boundary conditions are chosen to be prescribed forces, i.e., all flags equal to 2 (see Shelltech Report 88-2).

Under "Boundary Conditions" are NFOURT lines of input. The first NFOUR lines are considered in the analysis. Each line includes

NHARM: The first integer in the line is the harmonic number; 0 for axisymmetric, 1 for $\mathrm{n}=1$, etc.

ICS: $\quad$ The second integer should be set to 1 for a cosine harmonic, and 2 for a sine harmonic. In this case, the third line represents $\sin \left(2^{*}\right.$ theta) variation.

Boundary

Conditions: The next five real numbers are the prescribed edge conditions for the harmonic specified at the beginning of the line. In this case, the first line specifies an axisymmetric edge pull Ur and simultaneous axial translation Uz. The third line specifies an $n=2$ radial edge deformation of the same amplitude as the edge pull. Since $M s=0$ for all three harmonics (first entry of the five real values in each line), the edge is simply-supported, or hinged.

To specify the loading condition, assign pressure values to

PNOM: $\quad$ The nominal pressure load acting on the dish, in this case 689.7.

WINDAT: $\quad$ This is an array of pressure values from NPTSW equally spaced sample points which are distributed along the theta $=0$ meridian from the center $r$ $=0$ to the outer edge $r=R E$. The circumferential variation is assumed to be $\cos ($ theta), i.e., $n=1$. The program linearly interpolates the pressure between sample points in the radial direction. If no asymmetric pressure load is desired, set NPTSW and MESH to 0 and leave this array out. 


\subsection{Generation and Interpretation of Output}

When run, the DISH program reads the input file DISH.IN, and produces two output files: DISH.ECO, an echo of the input data which serves to verify that the input has been read properly, and DISH.DAT, an intermediate output data file. (See flowchart in Figure 1.)

\subsubsection{The Postprocessor DFCOMP}

Displacements and stresses for the deformed dish are obtained by running the program DFCOMP (Displacement and Force COMPutation). The executable DFCOMP.EXE is ob'tained by compiling the two FORTRAN files DFCOMP.FOR and SHAPE.FOR and linking the two object files. DFCOMP reads the data file DISH.DAT produced by the DISH program, as well as a small input file DFCOMP.IN, and produces an output file DFCOMP.OUT which lists the displacements, surface rotations, stress resultants, and moment resultants over a specified grid of points.

Example DFCOMP.IN and DFCOMP.OUT files are listed in Tables 2 and 3. The input parameters are

NPTS: $\quad$ Adjusts the density of the grid of output points. In this case, 4 points in the radial direction by 2 in the circumferential.

RANGE: The first two numbers under RANGE are the minımum and maximum $r$ values of the output interval. In this case, $r$ ranges from 6.0 to 7.5. The second two numbers are the minimum and maximum theta values (in degrees) of the output interval.

The logical flag ITER determines whether the grid is referenced to the undeformed or deformed dish. Note that the results of the DISH analysis are based on analytical solutions for which displacements and stresses are referenced to the undeformed dish. If ITER is set to .FALSE., $r$ and theta values have the conventional Lagrangian interpretation as the coordinates of a grid point on the undeformed dish. If set to .TRUE., $r$ and theta refer to a particular point in space, and an iterative process is invoked in order to evaluate the displacements and stresses at the point of the undeformed dish which ends up at the grid point ( $r$,theta) after deformation.

The DFCOMP.OUT file shown in Table 3 was generated using the DISH.DAT file corresponding to the input file DISH.IN of Table 1, and the DFCOMP.IN file of Table 2. DFCOMP.OUT lists 13 quantities at each of the grid points specified in the input file. The 13 quantities are the two rotations CHIs and CHIt of the normal (radians), the three displacements Ur, Uz, and Ut, the three moment resultants $\mathrm{Ms}, \mathrm{Mt}$, and Mst (moment per unit length), and the five stress resultants $\mathrm{Nr}, \mathrm{Nz}, \mathrm{Ns}, \mathrm{Nt}$, and $\mathrm{Nst}$ (force per unit length). The sign conventions are as depicted in Figure 2.

\subsubsection{The Subroutine SHAPE}

All the calculations pertaining to the deformed dish are performed by the subroutine SHAPE. The postprocessor DFCOMP is simply a driver which loops over a grid of $r$ and theta points, calling SHAPE for each point. If the output format of the DFCOMP processor is not satisfactory, another driver which calls SHAPE and writes the output in a different format can be created. 
The call parameters and operation of SHAPE are fully documented in the source code SHAPE.FOR. The first time SHAPE is called, it reads the data from the file DISH.DAT, and stores it in a common block. On subsequent calls, the data is already in memory, and can be rapidly accessed in order to evaluate displacements or stresses at different points.

\subsubsection{The Postprocessor DINFO}

Once the DISH program has been run and the DISH.DAT file has been created, the program DINFO, which reads the DISH.DAT file, can be run to obtain some basic information about the "membrane solution" and the deformed dish. The classical membrane solution of shell theory is the axisymmetric deformation for which the pressure load is carried entirely by membrane tensile forces, with no edge bending (see, e.g., Shelltech Report 87-1). Table 4 shows the output from DINFO, based on the DISH.DAT file corresponding to the input file DISH.IN of Table 1. The information is written to the screen, as well as to the file DINFO.OUT.

Referring to Table 4, it is seen that DINFO provides the "edge pull" and edge rotation of the membrane solution, the membrane solution center displacement relative to the edge, the focal length of the membrane solution shape, and the axial displacement of the edge which is required for the focal point to be the same as for the undeformed dish. Note that the information associated with the membrane solution is independent of the boundary conditions specified in DISH.IN. DINFO also provides the decay distances of the edge effects, as well as the pressurization parameter. A pressurization parameter much greater than one indicates a high degree of coupling between prestress and edge effects (see Shelltech Report 87-1).

\subsection{Ring/Membrane Interaction: Interface with Finite Element Programs}

In order to alleviate the computational demands of an integrated finite element structural analysis of the membrane and support structure, a hybrid analysis technique can be employed in which the support structure is modeled by finite elements and the membrane is modeled by the DISH program.

In this approach, the response of the ring and support structure to a given external loading is determined by a finite element analysis. The resulting deformations of the membrane support ring are then used as boundary conditions in the DISH program in order to determine the membrane response. If desired, the membrane edge forces computed by the DISH program can be fed back to the finite element analysis to obtain a first order correction to the loads on the support structure, which yields an updated ring deformation. This defines an iterative procedure for obtaining the solution to the coupled membrane/support structural problem. For reference while reading this section, the flowchart in Figure 3 shows the links between the primary programs and files involved.

\subsection{The Nominal State of the Assembled Concentrator Structure}

The total structure to be analyzed consists of a pressure stabilized paraboloidal membrane which is attached to a support structure such as the ring-spoke-centerpost design discussed by Kutscher et. al. (1988). Kutscher et. al. also point out that a small amount of "edge pull" can correct for local distortions near the edge, and that this edge pull is just that amount needed to obtain the membrane solution. 
Once the edge pull is applied to the pressurized dish, the new shape of the dish is very close to a paraboloid. The focal length of the deformed dish is increased, however, by the approximate factor $\mathrm{R} / \mathrm{S}$ relative to the focal length of the undeformed dish, where $\mathrm{R}$ is the ratio of the edge radius of the deformed dish to that of the undeformed dish, and $\mathrm{S}$ is the ratio of the edge slope of the deformed dish to that of the undeformed dish.

These observations suggest the design of a ring-spoke-centerpost support structure with the radius of the undeformed ring and pretension of the spokes chosen such that the membrane tension contracts the ring to the radius specified by the membrane solution. The final picture is of a pressure-loaded dish and tensioned support structure in equilibrium, with the dish held in the shape given by the membrane solution. The receiver would be located at the focal length of the deformed dish, which is somewhat greater than that of the undeformed dish. The support structure should be designed such that external loads do not cause appreciable perturbations relative to this state, which will be referred to as the "nominal state" of the entire structure.

\subsection{Fourier Analysis of Finite Element Nodal Data}

In order to evaluate the effect of ring deformation on dish shape, the discrete nodal displacements and rotations from the finite element ring/support structural analysis must be converted to harmonic coefficients which are suitable for input to the DISH program. This is achieved by means of the program FSCOEF. The executable FSCOEF.EXE is obtained by compiling and linking the source files FSCOEF.FOR and FOURIER.FOR. (The subroutine FOURIER in the file FOURIER.FOR performs the discrete Fourier analysis.)

The input to FSCOEF is the file FSCOEF.IN, which should contain the nodal displacements in the $r$, theta, and $z$ directions and the nodal rotations about the $r$, theta, and $z$ axes arranged as six columns of data. The first row of six numbers corresponds to the nodal displacements and rotations at theta $=0$. The second row of six numbers corresponds to the adjacent node, moving around the dish in a counter-clockwise direction, as seen looking into the dish. The total number of rows of data is therefore equal to the number NPTS of circumferential nodes around the ring. For the purposes of this input file, theta is defined as counter-clockwise looking into the dish, since most finite element models will be constructed with this convention.

The output file FSCOEF.OUT contains the lines to be inserted under "Boundary Conditions" in the input file DISH.IN to the DISH program. FSCOEF computes the Fourier cosine and sine coefficients for all harmonics up to $\mathrm{N}=\mathrm{NPTS} / 2$, which is the highest harmonic which can be reasonably computed based on NPTS circumferential nodes. In practice, the harmonic coefficients for $\mathrm{N}$ greater than NPTS/4 are usually not very accurate. (For a symmetric analysis, the Fourier sine coefficients (ICS $=2$ ) will all be negligibly small, so that the lines corresponding to these harmonics can be deleted.)

The DISH.IN file is then created, with NFOURT equal to the total number of lines included under "Boundary Conditions." All five Boundary Condition Flags should be set to 1 for prescribed displacements. The DISH program can then be run to produce the DISH.DAT file, and the postprocessor DFCOMP, described in Section 2.3.1, can be run in order to compute displacements and stresses in the dish. 


\subsection{Computation of Membrane Reaction Forces}

The postprocessor FCOMPN, which works similarly to DFCOMP, can be used to compute the nodal reaction forces and moments which the membrane exerts on the ring. The source code FCOMPN.FOR should be compiled and linked with SHAPE.OBJ. When run, FCOMPN reads the short input file FCOMPN.IN and the data file DISH.DAT, and computes the nodal forces and moments by "lumping" the continuous resultant forces and moments at the nodes. The nodal forces and moments are written to the file FCOMPN.OUT.

In the input file FCOMPN.IN, ITER should be set to .FALSE.; the number of grid points in the $r$ and theta directions should be set to $l$ and the number of circumferential nodes, respectively; the upper and lower limits for $r$ should be set to the dish radius; and the upper and lower limits for theta should be set to the theta angles of the first and last nodes around the edge of the dish. The unit conversion factors FORFAC and MOMFAC are scalar multipliers for the forces and moments, and should be set to 1.0 if no scaling is desired.

\subsection{Example: Ring Deformation from a SUPERSAP Analysis}

As an example, consider the dish frame analysis by Sallis (1988), for a frame with hinge points at plus and minus 67.5 degrees subjected to a head-on wind load. The ring displacements from run SDF4E, which was re-run with SUPERSAP as run ASDF4E, are listed in Table 5. The integers in the first column of Table 5 are the node numbers around the perimeter of the ring where the dish is attached. Node 1 is at theta $=0$ node 9 is at theta $=-90$ (in Figure 2), and node 17 is at theta $=-180$. Only one half the frame was analyzed due to symmetry.

\subsubsection{Fourier Analysis of SUPERSAP Output}

The last six columns in Table 5 are the displacements (in inches) and rotations (in degrees) relative to a Cartesian coordinate system, where the $\mathrm{x}$ and $\mathrm{y}$ axes are at theta = -90 and -180 in Figure 2. The preprocessor R2P can be used to convert this data to the polar coordinate form required for input to FSCOEF. When run, R2P will first ask whether the ring displacement data is from a half-model symmetric analysis. If so, then R2P will compute the displacements and rotations for the remaining half of the ring from symmetry considerations. R2P then asks if unit conversion is desired. If so, the two unit conversion factors for the displacements and rotations are read from the file R2P.DAT. In this case, R2P.DAT contains the two numbers 0.0254 , to convert from inches to meters, and 0.0174533 , to convert from degrees to radians.

$\mathrm{R} 2 \mathrm{P}$ reads the displacements and rotations in Table 5 from the file R2P.IN, and produces the file R2P.OUT, which is the $r$, theta, and $z$ nodal displacements and $r$, theta, and $z$ nodal rotations arranged as six columns. The file R2P.OUT, shown in Table 6, is then renamed FSCOEF.IN, and the program FSCOEF is run in order to obtain the file FSCOEF.OUT, which contains the Fourier coefficients of the displacements and rotations (see the flowchart in Figure 3). The file FSCOEF.OUT is shown in Table 7. In each line, the first integer is the Fourier harmonic number, the second integer indicates a cosine (ICS $=1$ ) or sine (ICS = 2) harmonic, and the next five numbers are Fourier coefficients. Note that in this case, because the problem is symmetric, the sine terms are negligible so that all the lines with ICS $=2$ can be deleted. 


\subsubsection{Preparation of Input to DISH}

The file FSCOEF.OUT, with the sine terms removed, is imported directly into the "Boundary Conditions" section of the file DISH.IN, which appears as shown in Table 8. In this case, a 3 mil thick steel membrane is chosen. Note that although NFOURT must be set to 17, which is the number of lines of boundary condition data, NFOUR is set to 9, so that only the harmonics up to $\mathrm{N}=8$ will be used. For 32 circumferential nodes, the Fourier coefficients beyond about the eighth harmonic are not very accurate. Figure 4 illustrates the accuracy with which the Fourier series through $N=8$ reproduces the SUPERSAP displacements in the $z$ direction at the edge of the dish.

For the Boundary Condition line corresponding to $N=0$ in Table 8 , the coefficients for Ur and $\mathrm{Uz}$ have been modified in order to account for edge pull and the new focal point of the membrane solution. The program DINFO was run to determine the edge pull Ur and edge translation Uz required to give the "membrane solution" shape with the same focal point as the undeformed dish, and these values were added to the SUPERSAP values in order to represent the fact that the support structure would be designed with pretension and ring radius such that the edge pull is adjusted properly in the nominal state with no external loads. Although spoke pretension was not included in the SUPERSAP model, the associated uniform radial displacement would just be linearly superposed on the results in Table 5. The DISH program is now run with the input file DISH.IN shown in Table 8, and the DISH.DAT file is produced.

\subsubsection{Computation of Nodal Loads}

In order to determine the nodal loads, the postprocessor FCOMPN is run, with the input file FCOMPN.IN shown in Table 9. In this case, loads are needed only for the 17 nodes halfway around the ring. Also, the sign convention for theta used by FCOMPN is opposite to that in Figure 2, in order to conform to the convention in the finite element node numbering. To convert from the MKS units used in the DISH analysis, the unit conversion factors FORFAC and MOMFAC in FCOMPN.IN were taken to be 0.22472 and 8.84723 for conversion from Newtons to pounds and from Newton-meters to inch-pounds.

The output file FCOMPN.OUT shown in Table 10 contains the force and moment reaction loads that the membrane exerts on the ring. Note that these loads are opposite in sign to the loads that the the ring exerts on the membrane, and that in applying these loads to the finite element model, the loads at nodes 1 and 17 should be divided by two due to symmetry.

Figure 5 shows plots of the meridional stress resultant Ns and the tangential stress resultant Nst at the edge of the dish due to the deformation under consideration, which includes Fourier harmonics through $N=8$. The stress resultant Ns oscillates about a constant value of about $6700 \mathrm{~N} / \mathrm{m}$, which is the prestress value of the nominal membrane solution. Near the hinge point at theta $=67.5$, the stress is higher, due to bending of the ring about the hinges, but on either side of the hinge point, the stress drops below the nominal value. Also note that the edge forces for a steel membrane (solid lines in Figure 5) are significantly greater than those for a composite membrane (dotted lines).

\subsubsection{Perturbations about the Nominal State}

The nodal loads given in Table 10 are the total loads which the membrane exerts on the ring. From the comments of Section 3.1, however, it can be inferred that the frame will be designed in such manner so that in the nominal state with no external loads, the force 
exerted on the dish by the ring is exactly that which is necessary to maintain the paraboloidal "membrane solution" shape. This nominal value of tension would be achieved by means of a small increase in the radius of the undeformed ring, combined with pretensioning of the spokes. These effects, though not included in the SUPERSAP analysis, could be included easily by linear superposition.

In order to account for the fact that these "DC" terms have been omitted from the finite element analysis, only the difference between the membrane reaction forces of Table 10 and the reaction forces of the membrane solution should be fed back to the finite element model. This yields a more realistic view of how the membrane and support structure will interact if the edge pull is properly adjusted in the nominal state.

In order to obtain the change in load relative to the membrane solution, the DISH program is run a second time, but with the input file DISH.IN shown in Table 11 . This input file specifies the membrane solution, since the radial edge displacement in the boundary conditions is adjusted to the proper edge pull for the membrane solution. Now the program FCOMPN is run in order to obtain the nodal loads associated with the membrane solution.

To obtain the difference in loads relative to the membrane solution, run the program FSUBTR (Force SUBTRact). FSUBTR takes two input files FSUBTR.INI and FSUBTR.IN2 of the form shown in Table 10, and subtracts the loads of the second from those of the first, and writes the resulting changes in loads to FSUBTR.OUT. With FSUBTR.IN1 chosen to be the file shown in Table 10, and FSUBTR.IN2 chosen to be the nodal loads of the membrane solution corresponding to the DISH.IN file of Table 11, the file FSUBTR.OUT shown in Table 12 is obtained. The effect of feeding back these incremental membrane reaction forces to the finite element model is discussed by Schreiner (1988).

\subsection{Coupled Optical/Structural Analysis using OPTDSH and DISH}

The ray trace program OPTDSH is capable of a non-axisymmetric optical analysis of a deformed paraboloidal membrane, using shape information from the DISH structural analysis program. OPTDSH computes the paths of incoming rays which reflect off the dish surface, and provides graphical information describing the focusing characteristics of the deformed dish. More detailed documentation pertaining to the structure and features of the OPTDSH program is provided in Appendix A.

\subsection{Interface between the Optical and Structural Codes}

The interface between the OPTDSH optical code and the DISH structural code is based on the subroutine SHAPE described in Section 2.3.2. Once the DISH program is run for a particular configuration, boundary conditions, and loading, a DISH.DAT file is produced which contains all the information necessary for computing shape information. When the OPTDSH code needs shape information at a particular point of the dish, it calls the subroutine SHAPE, which in turn accesses the data in DISH.DAT. The speed of the ray trace operation depends on the number of harmonics included in the structural model, but a typical analysis with several thousand rays can be carried out in a few minutes on a 286 or 386-based computer. 


\subsection{Examples}

Examples to be discussed include the effects of pressure changes on the membrane and prescribed deformations of the edge. In the following examples, the undeformed membrane is taken to have a radius of $7.5 \mathrm{~m}$, a thickness of $10 \mathrm{mils}$, a focal length of 9 $\mathrm{m}$, a modulus of $7.93 \mathrm{GPa}$, and Poisson's ratio 0.3. The stabilization pressure is taken to be $689.7 \mathrm{~Pa}$, and the appropriate edge pull and axial translation are included so that the nominal state of the dish corresponds to the membrane solution, with the same focal point as for the undeformed dish. All graphical output is produced by OPTDSH, based on a field of randomly distributed incident rays which are parallel to the dish axis.

\subsubsection{Head-on and Side Wind Loads}

Consider the case of wind blowing head-on into the dish. As a first approximation, this situation can be represented by a uniform increase in the stabilization pressure. For the present analysis the value $72.8 \mathrm{~Pa}$ corresponding to a $27 \mathrm{mph}$ wind was used; this value was obtained from Sallis (1988). The DISH program was therefore run with a stabilization pressure of $762.5 \mathrm{~Pa}$, keeping the edge pull fixed at the value corresponding to $689.7 \mathrm{~Pa}$. The resulting DISH.DAT file was used by OPTDSH in order to compute the ray trace results shown in Figure 6.

Figure 6 is a spot diagram which indicates where parallel incident rays hit the focal plane. The shape of the symbol indicates the radial interval on the dish from which the ray was reflected. For example, the star symbols indicate rays which hit the dish at a radius between $6 \mathrm{~m}$ and $7.5 \mathrm{~m}$. In this case, the rays which stray the furthest from the center of the focal spot bounced off the dish within $1.5 \mathrm{~m}$ of the edge. This is due to the fact that the greatest slope changes occur near the edge. The maximum radius and $\mathrm{rms}$ radius of the focal spot are $13.1 \mathrm{~cm}$ and $4.4 \mathrm{~cm}$ respectively. For comparison, the stabilization pressure of $689.7 \mathrm{~Pa}$ with no edge pull yields a focal spot with an rms radius of $45.4 \mathrm{~cm}$.

Now consider the case of wind blowing from the side. The pressure load was taken to be the nominal stabilization pressure $(689.7 \mathrm{~Pa})$ plus the cos(theta) wind load distribution specified under WINDAT in Table 1 . Note that the wind pressure varies from zero in the center to a maximum of $72.8 \mathrm{~Pa}$ at the edge. The resulting focal spot, computed by OPTDSH, is shown in Figure 7. Note the "coma" effect due to the non-axisymmetric deformation. Since theta $=0$ is on the right in Figure 7, the right side of the dish is under higher pressure than the left, causing the focal spot distortion shown. The maximum and rms radii of the focal spot are of the same order of magnitude as for the head-on wind load (see Figure 6).

\subsection{2 $\mathrm{N}=2$ Edge Displacement}

The effect on the focal spot of a $\cos \left(2^{*}\right.$ theta) radial edge displacement of amplitude 1 $\mathrm{cm}$ is shown in Figure 8. Again, the rays which strike the focal plane furthest from the center are the ones which bounce off the edge of the dish. Note that the greatest error is at multiples of 90 degrees, where the maximum slope changes of the dish surface occur. The rms spot radius of $16.2 \mathrm{~cm}$ indicates that this is an undesirable deformation from the standpoint of optical efficiency. 


\subsubsection{Ring Deformation from a SUPERSAP Analysis}

If the DISH program is run with the prescribed edge deformation from the SUPERSAP analysis as discussed in Section 3.4, a DISH.DAT file is produced which can then be used by OPTDSH to determine the optical effect of the ring distortion. The focal spot diagram is shown in Figure 9. The distortion is clearly non-axisymmetric, due to bending about the hinge points at plus and minus 67.5 degrees. The rms spot radius, however, is only $5 \mathrm{~mm}$, which indicates that the frame is effective in preventing the edge of the dish from deforming. In fact, the effect of the ring distortion is negligible compared to that of the overall pressure increase due to the head-on wind load (see Figure 6).

A second type of plot produced by OPTDSH is the "inverse" of the spot diagram, which is shown in Figure 10. This plot shows the dish surface, and the symbols indicate the region of the focal plane which is struck by incident rays from that point of the dish. The plus sign symbols in Figure 10 indicate rays which are reflected to a point very near the center of the focal plane, and the star symbols indicate rays which stray the furthest from the center. From Figure 10, it is evident that the left side of the dish is relatively undisturbed, with most of the rays directed toward the focus. There is substantial deformation near the hinge points, however, and rays which strike near the hinge points are deflected away from the center of the focal plane.

\subsection{Work in Progress: Integrated Thermal/Optical/Structural Analysis}

Current effort focuses on developing the capability to carry out ray trace calculations for incident rays which are not parallel to the dish axis. A subroutine RADISH has been implemented which can determine the intersection point between the deformed dish and an arbitrary ray. RADISH utilizes a rapidly convergent Newton-Raphson type algorithm, and relies on the subroutine SHAPE.

The capability to trace off-axis rays, when included in the OPTDSH program, will enable modeling of additional effects such as sun size and random surface slope errors. Once these effects are incorporated, the optical/structural analysis can be interfaced with the thermal efficiency analysis, which models the absorption and loss of energy at the collector.

\subsection{Conclusions}

The DISH program for the axisymmetric and non-axisymmetric structural analysis of paraboloidal dish membranes has been implemented and documented, and an interface to optical analysis software has been provided, based on the subroutine SHAPE and the data file DISH.DAT.

Software has been developed for transforming nodal displacements and rotations from finite element programs to the Fourier coefficients required for input to the DISH program. Software has also been developed for transforming the stress and moment resultants computed by the DISH program to nodal forces and moments, which can be applied as membrane reaction loads in a finite element model.

Preliminary optical/structural analyses have been carried out. It has been observed that the dish shape corresponding to the "membrane solution" of shell theory is very close to a paraboloid, with a focal length which is somewhat longer than that of the undeformed dish. For the load case and dish frame considered in run SDFiE of the finite element analysis by Sallis (1988), analyses based on DISH and OPTDSH indicate that the ring 
distortions do not have a significant optical effect, since the resulting rms focal spot size is on the order of a few millimeters. On the other hand, the distortions produced by overall pressure changes due to wind can lead to an rms focal spot size on the order of a few centimeters, which is non-negligible when compared to other unavoidable sources of error such as sun size.

\section{REFERENCES}

Balch, C. D. and Steele, C. R. (1988). "Nonaxisymmetric deformations of thin paraboloidal shells with initial prestress," Shelltech Report 88-2.

Kutscher, C., Lewandowski, A., Wood, R., Wendelin, T., and Jorgensen, G. (1988). "Membrane Dish Research Task FY 1987 Progress Report," Solar Energy Research Institute, Golden, CO.

Sallis, D. V. (1988). "Cable reinforced dish frame cable design studies summary report," Rep. R340588A, DAN-KA Products Inc., Englewood, C0.

Schreiner, R. J. (1988). "Cable reinforced dish frame membrane force effect," Rep. R340888B, DAN-KA Products Inc., Englewood, C0.

Steele, C. R., and Balch, C. D. (1987). "Edge effects in a shell of revolution under pressure load," Shelltech Report 87-1. 
Table 1: Sample input file DISH.IN for the DISH program.

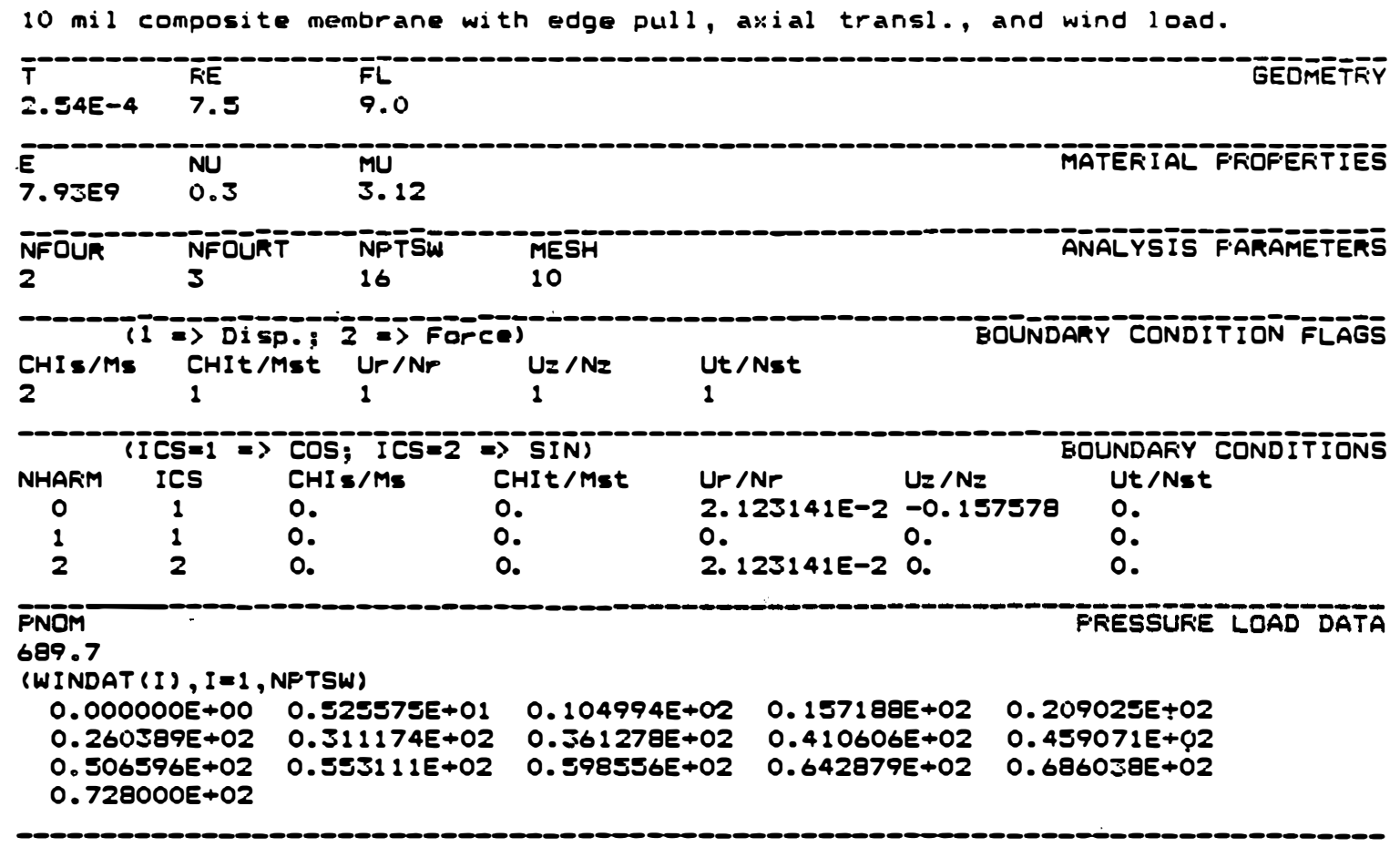

\section{Table 2: Sample input file DFCOMP.IN for the post-processor DFCOMP.}

Input File DFCCIMF. IN, for the postprocessor DFCOMF.

\begin{tabular}{|c|c|c|c|}
\hline $\begin{array}{l}\text { I TER } \\
\text {. FALSE. }\end{array}$ & $\begin{array}{l}\text { NFTS } \\
4,2\end{array}$ & $\begin{array}{l}\text { RANGE } F \text {, } \\
6.0,7.5\end{array}$ & $\begin{array}{l}\text { theta(d) } \\
0 ., 90 .\end{array}$ \\
\hline
\end{tabular}


Table 3: Sample output file DFCOMP.OUT for the post-processor DFCOMP.

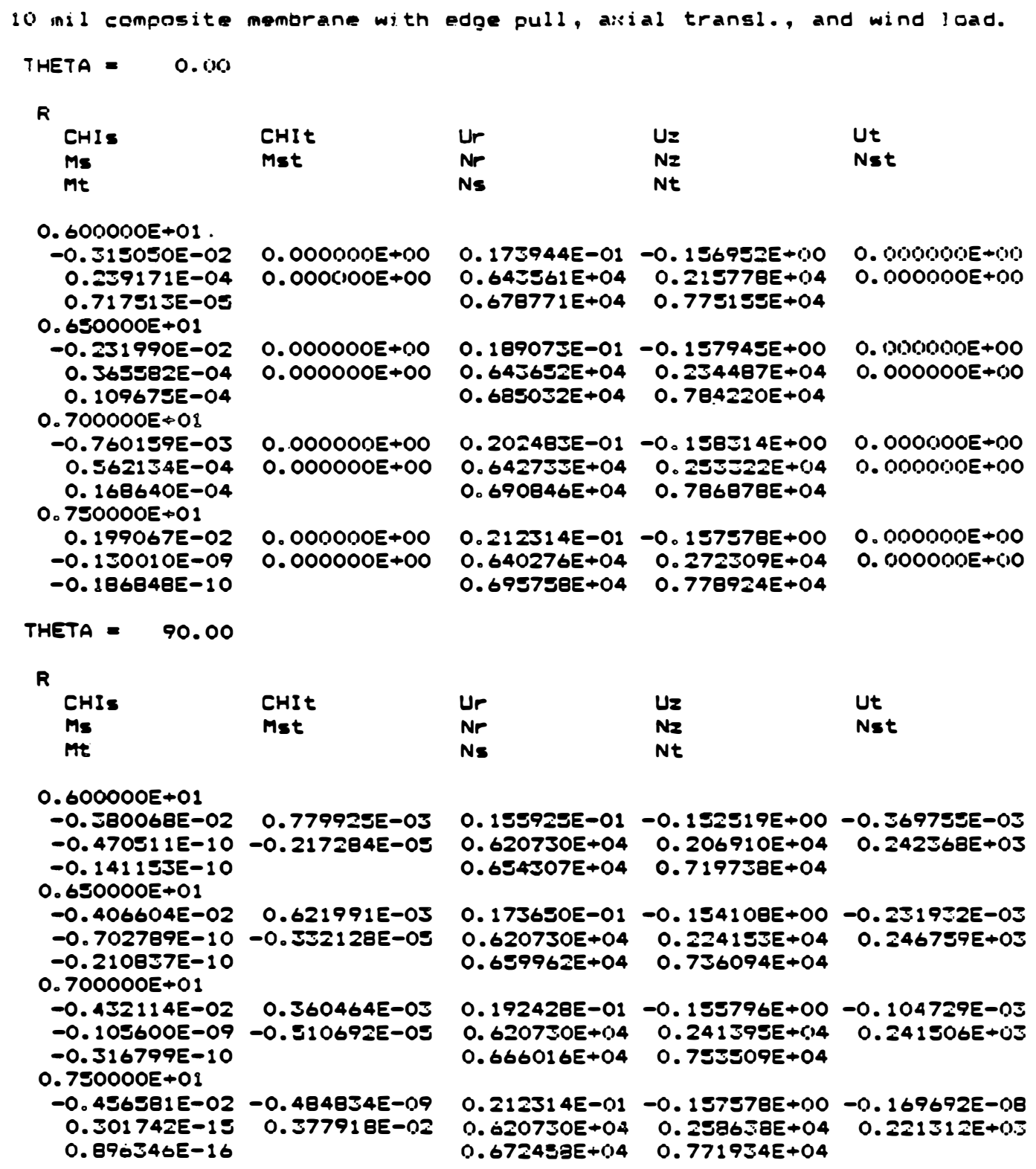


Table 4: Output from the postprocessor DINFO.

10 mil composite membrane with edge pull, axial transl., and wind load.

Memorane Solution Data:

Fiadial edge displacenient .................. $=0.212 .14 E-01$

Meridional edoe rotation ............................

Axlal displacement at center ............ UZe = $0.147406 E-(11)$

Approximate focal length ............ FLriew = 0.914284E+(01

Edge displacement for same focus ...... UZe $=-0.157578 E+00$

Edge Effect Data:

Short decay distance .............. DELTAS = 0.417920E-0.02

Long decay distance ................ DELTAL = $0.5590975+(011$

Fressurization parameter ................ RHO = $0.423467 E+(03$

Decay distance for zero pressure..... DELTA = 0.1720100E+00

\section{Table 5: Nodal displacements and rotations from a SUPERSAP analysis.}

$.0000+00$

$-4.15 \div 5 E-0.4$

$-5.9311 E-1.14$

$-4.6802 E-104$

$-2.034 .5 E-(14)$

$-1.259+E-(0) 4$

- $5.5877 E-03$

$-8.8171 E-03$

$-5.5974 E-03$

$-2.8290 E-03$

$-1.2870 E-03$

$-3.2236 E-04$

2.721 SE-04

5.5920E-04

5. $5985 E-04$

3. $3294 E-104$

$.0000 E+00$
$2.8422 E-0.3-2.6178 E-02-1.9085 E-02$ $2.4824 E-0.3-2.8155 E-02-1.2755 E-02$

1.520-E-03 - \$.2760E-02 7.975EE-03

3.5.70E-04 - $3.5450 E-02 \quad 3.6797 E-02$

$-0.9044 E-04-2.9252 E-02$ 4.5752E-02

$-1.5410 \mathrm{UE}-0 \mathrm{3}-1.2164 \mathrm{E}-02-2.5520 \mathrm{OE}-0 \mathrm{3}$

$1.0(.146 E-0.7-1.4145 E-0.7-6.6068 E-0.2$

$1.5504 E-03-2.2(127 E-02-3.0882 E-02$

$1.9951 E-05-5.0110 E-02-2.2748 E-02$

$2.623 .3 E-0.0-6.7805 E-02-2.1650 .0-02$

$3.4608 E-03-7.5856 E-0.2-1.3089 E-02$

4. $3780 E-03-8.0142 E-02-4.5930 .0-03$

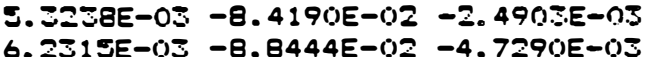

$6.9835 E-03-9.2099 E-02-7.0824 E-03-3.0644 E-04-5.3321 E-04$

$7.4746 E-03-9.4462 E-02-7.9801 E-03-1.7080 E-05-2.6859 E-04$

$7.6442 E-03-9.5265 E-6.52-8.0856 E-03$
$.0000+00$ (i)E
.
$2.0576 E-02-1.0021 E-10$
$2.7410 E-02-1.025 B E-0.5$
$-0181 E-02 \quad 1.0659 E-10$
$1.2652 E-0.0$
4. $7994 E-02-4.2994 E-0.5$
4. $.570 E-02-1.9262 E-0.3$
$1.5650 E-02-1.4659 E-0.3$
5. $8971 E-044-1.1=21 E-03$ 


\section{Table 6: Input file FSCOEF.IN to the Fourier analysis program FSCOEF.}

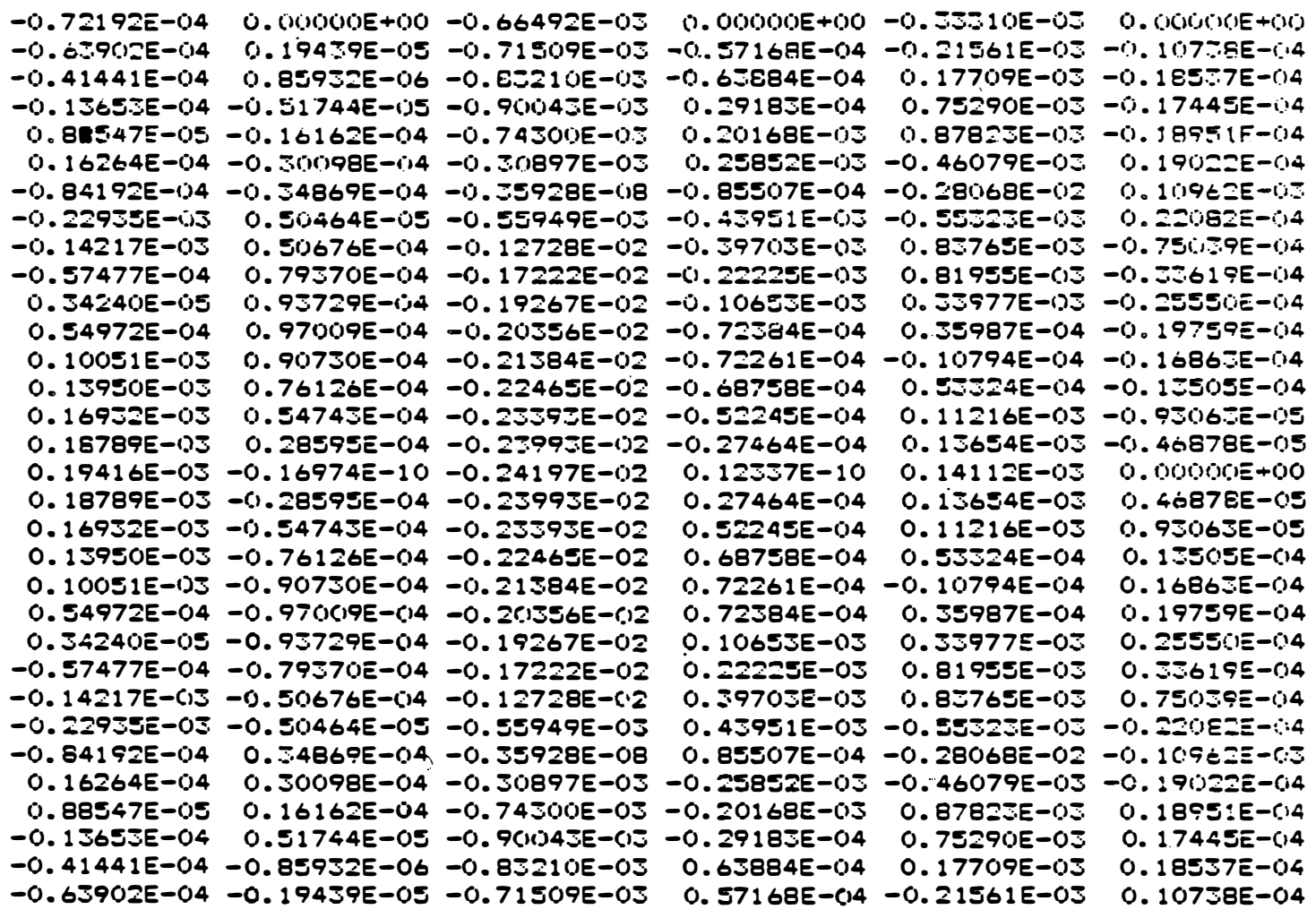


Table 7: Output file FSCOEF.OUT from the Fourier analysis program FSCOEF.

\begin{tabular}{|c|c|c|c|c|c|c|}
\hline 0 & & $E-0.99$ & 11 & 5 & $=-0-2$ & -6 \\
\hline$\cdot 1$ & 1 & ב & $0 \Xi$ & & 0 & $i$ \\
\hline 1 & & $E-10$ & 10 & -0 & $0.1212-12$ & $-1, .5$ \\
\hline & & & $0.738268 E-0.44$ & & -0 & -0 \\
\hline & & $E-10$ & -0.10 & $398 E-10$ & -0.5 & -11 \\
\hline & & $=-0.3$ & & -0. & & \\
\hline & & -09 & & & -0 & -0 \\
\hline & & 64 & & & 14 & $a$ \\
\hline & 2 & $E-10$ & 10 & $-\dot{0} .2 r$ & $0 .=$ & $-\infty$ \\
\hline & & -0 & & & בَ & 0.5 \\
\hline & & 9 & () & & & \\
\hline 6 & & -0 & -0 & & & $-i$. \\
\hline & & -1.99 & 0 & $E-10$ & 0. & $0.2 \varepsilon$ \\
\hline & & $=0 \Xi$ & 0. & 0.4 & -0 . & -0. \\
\hline & & 9E-09 & -0.2052 & -10 & 0.1 & 0. \\
\hline & & -03 & & & & \\
\hline & & -6 & & & & \\
\hline & 1 & $462 E-03$ & 0 & -0 & -0 & 0.2426105 \\
\hline & & $E-09$ & -0 . & -0 & 10 & i ZSE \\
\hline & & $641 E-03$ & -0 & $-\bullet$ & 04 & 96 \\
\hline & & उÜE-09 & 0.14 & -0.1 & 0.5 & -0 . \\
\hline & 2 & $E-0 \Xi$ & -0 & & 04 & -0 \\
\hline & 2 & $=-09$ & 0 & 10 & 10 & 12 \\
\hline & & -0 & & & & -0 \\
\hline & & & & & & 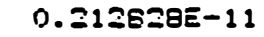 \\
\hline & & 3691 E-03 & 0 & & -0 & -0 \\
\hline & & $1794 E-09$ & & & & -0 \\
\hline & 2 & 07919E-0I & 6461 & -0 & OS & $0.592670 \mathrm{E}$ \\
\hline & 2 & 708E-0.9 & $0.152310 E$ & $158 E-11$ & $4080 E-10$ & $904 E-12$ \\
\hline & 1 & S86E- & -0.56 & 0.5 & $8880 E-0.05$ & $0.595012=E-1.6$ \\
\hline & & 0 & & & & -0. \\
\hline & 1 & $\begin{array}{l}-03 \\
-09\end{array}$ & $\begin{array}{l}36 E-11 \\
O E-11\end{array}$ & $\begin{array}{l}=05 \\
-10 \\
-10\end{array}$ & $\begin{array}{l}84 \\
10\end{array}$ & 12 \\
\hline & & & & & & \\
\hline
\end{tabular}




\section{Table 8: Input file DISH.IN with prescribed edge displacements from FSCOEF.OUT.}

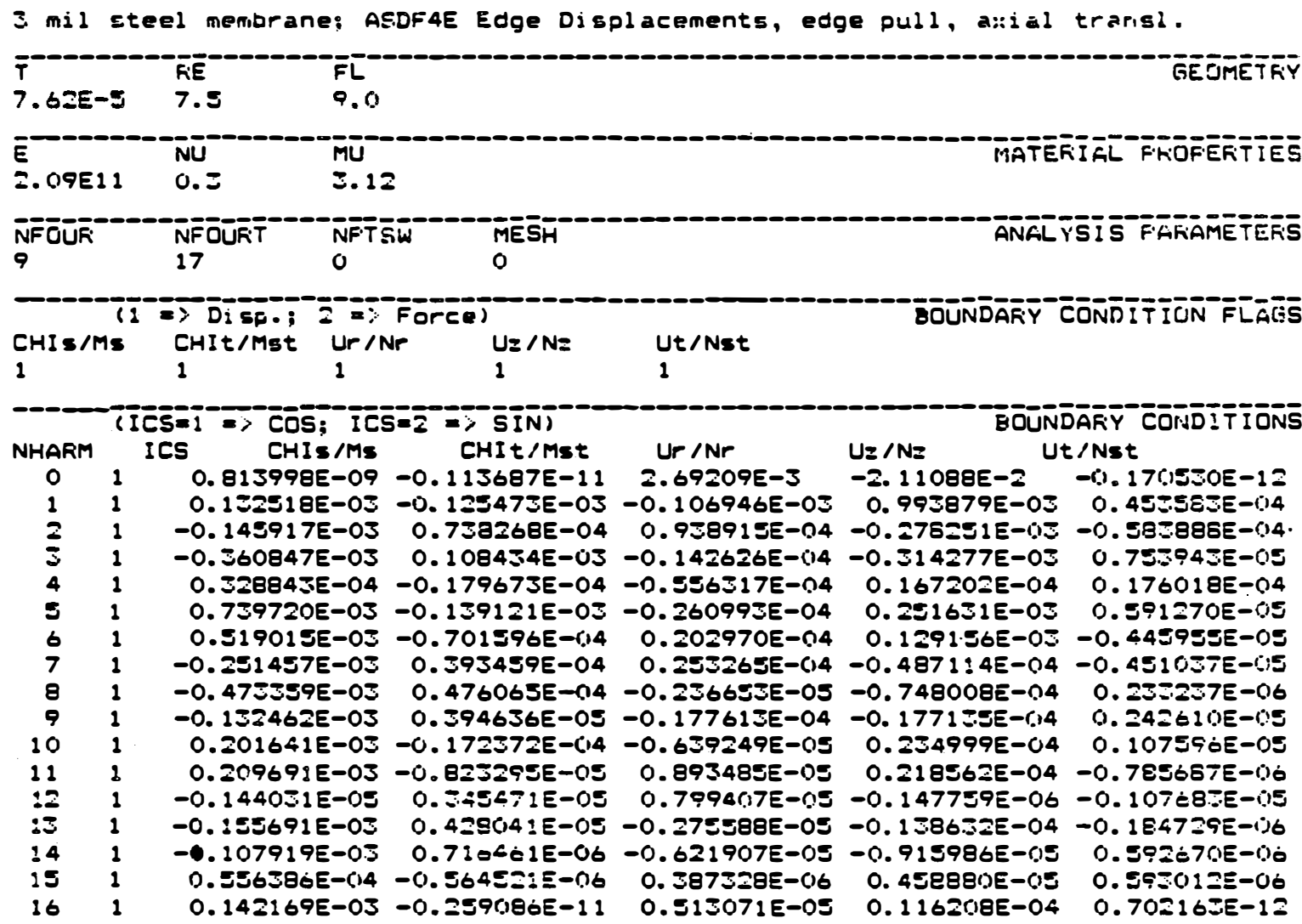

F̄NŌM

689.7

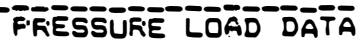




\section{Table 9: Input file FCOMPN.IN to the program FCOMPN.}

Input File FCOMFN. IN, for the postpracessor FCDMFN.

\begin{tabular}{|c|c|c|c|c|c|}
\hline & $\begin{array}{l}\text { NFTS } \\
1,17\end{array}$ & $\begin{array}{l}\text { FANGE } r \text {, } \\
7.5,7.5\end{array}$ & $\begin{array}{l}\text { theta }(d) \\
0 ., 180 .\end{array}$ & $\begin{array}{l}\text { FOFIFAC } \\
0.22472\end{array}$ & $\begin{array}{l}\text { MOMFAC } \\
\text { B. } 8472=1\end{array}$ \\
\hline
\end{tabular}

Table 10: Output file FCOMPN.OUT of nodal forces and moments from FCOMPN.

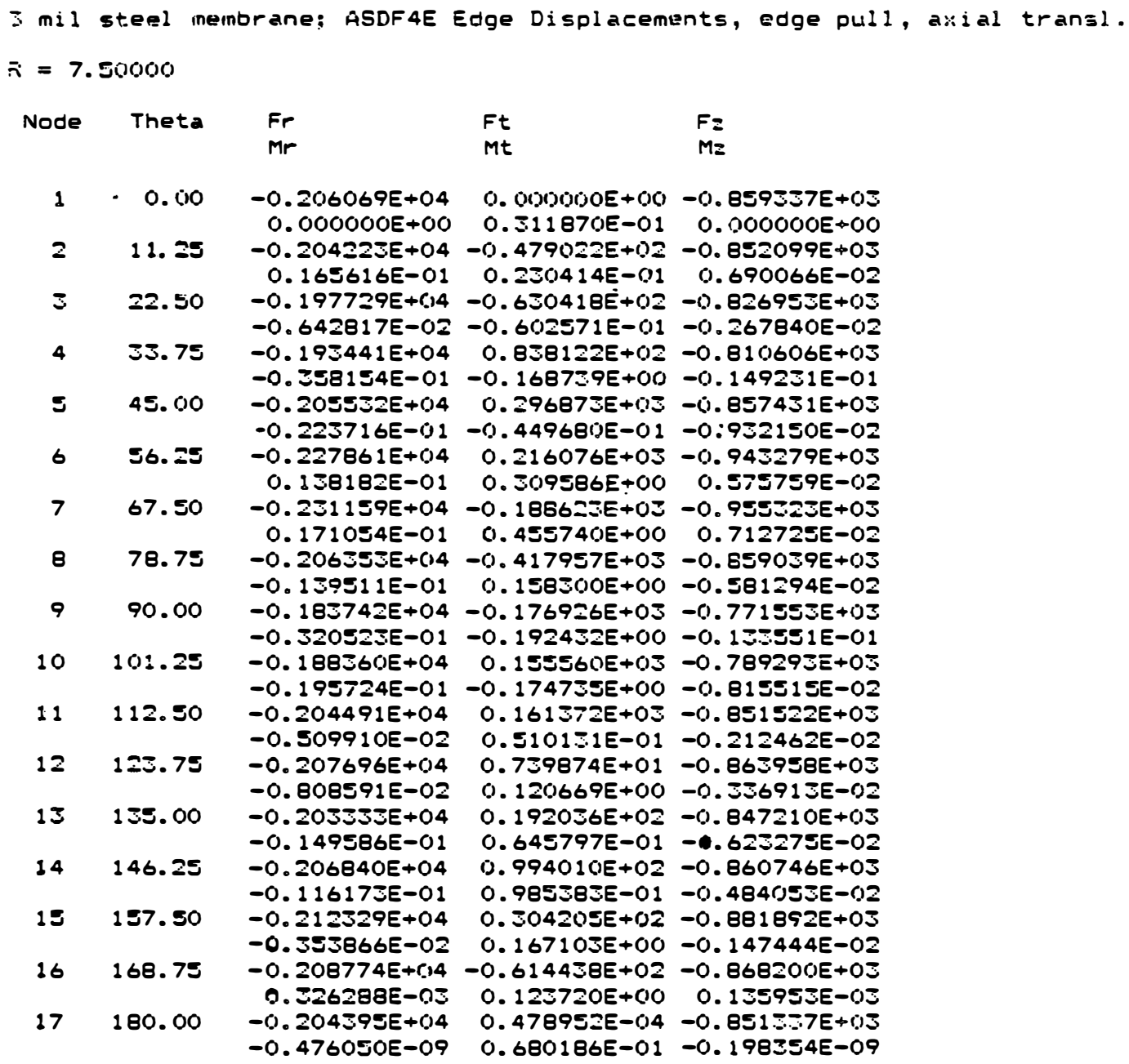


Table 11: Input file DISH.IN for membrane solution only.

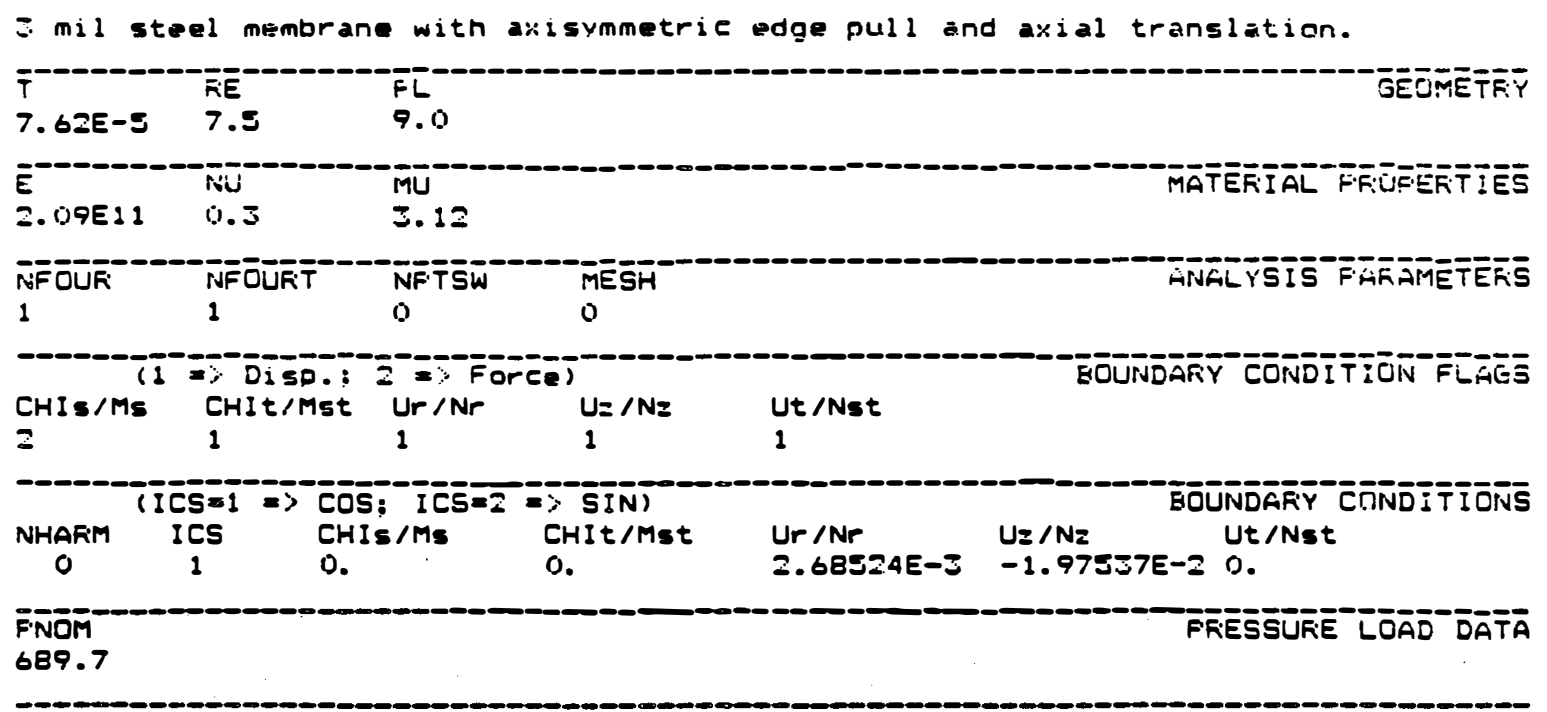


Table 12: Change in nodal forces and moments relative to the membrane solution, from FSUBTR.

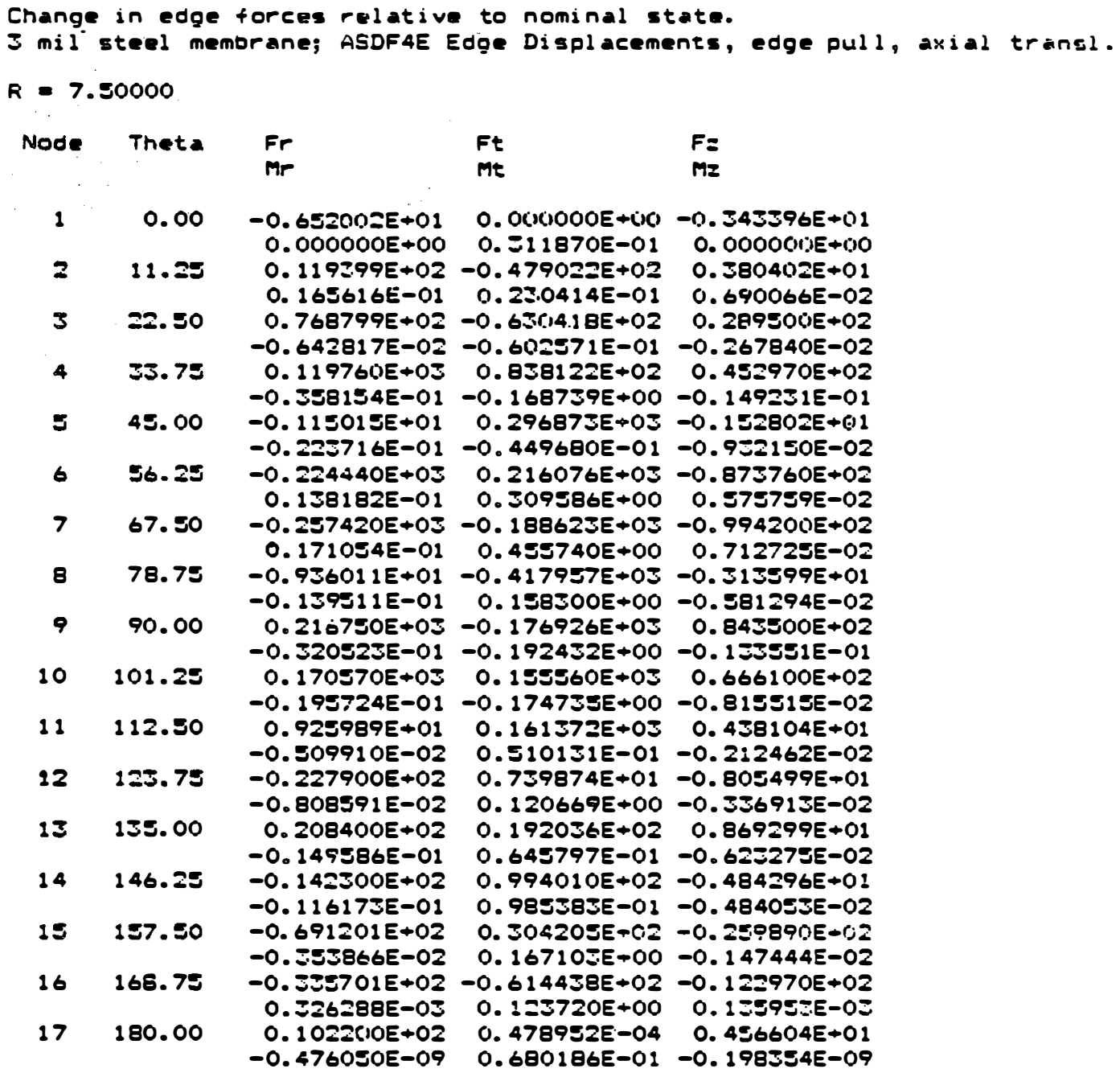




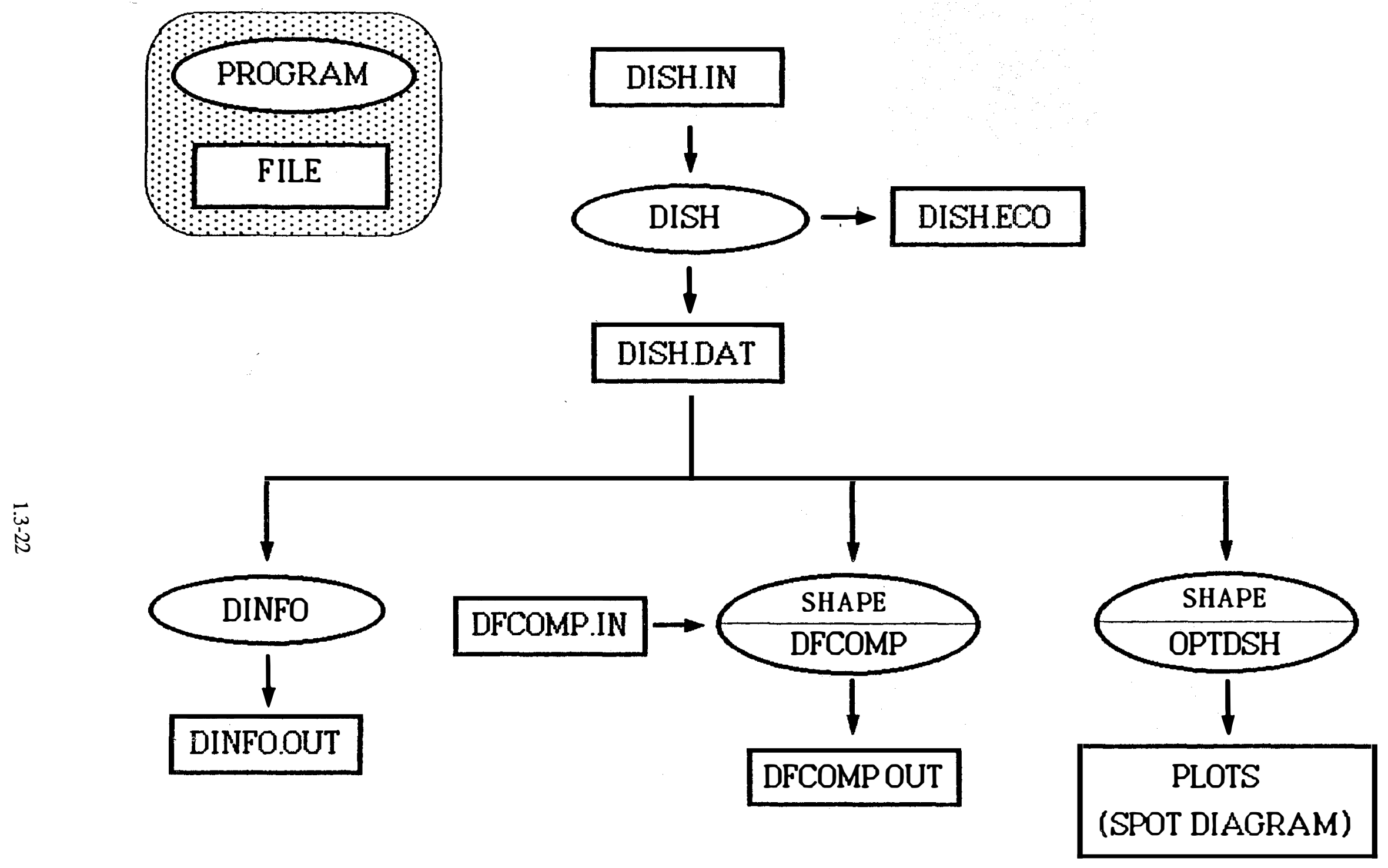

Figure 1: Flowchart of data paths for structural and structural/optical analyses. 

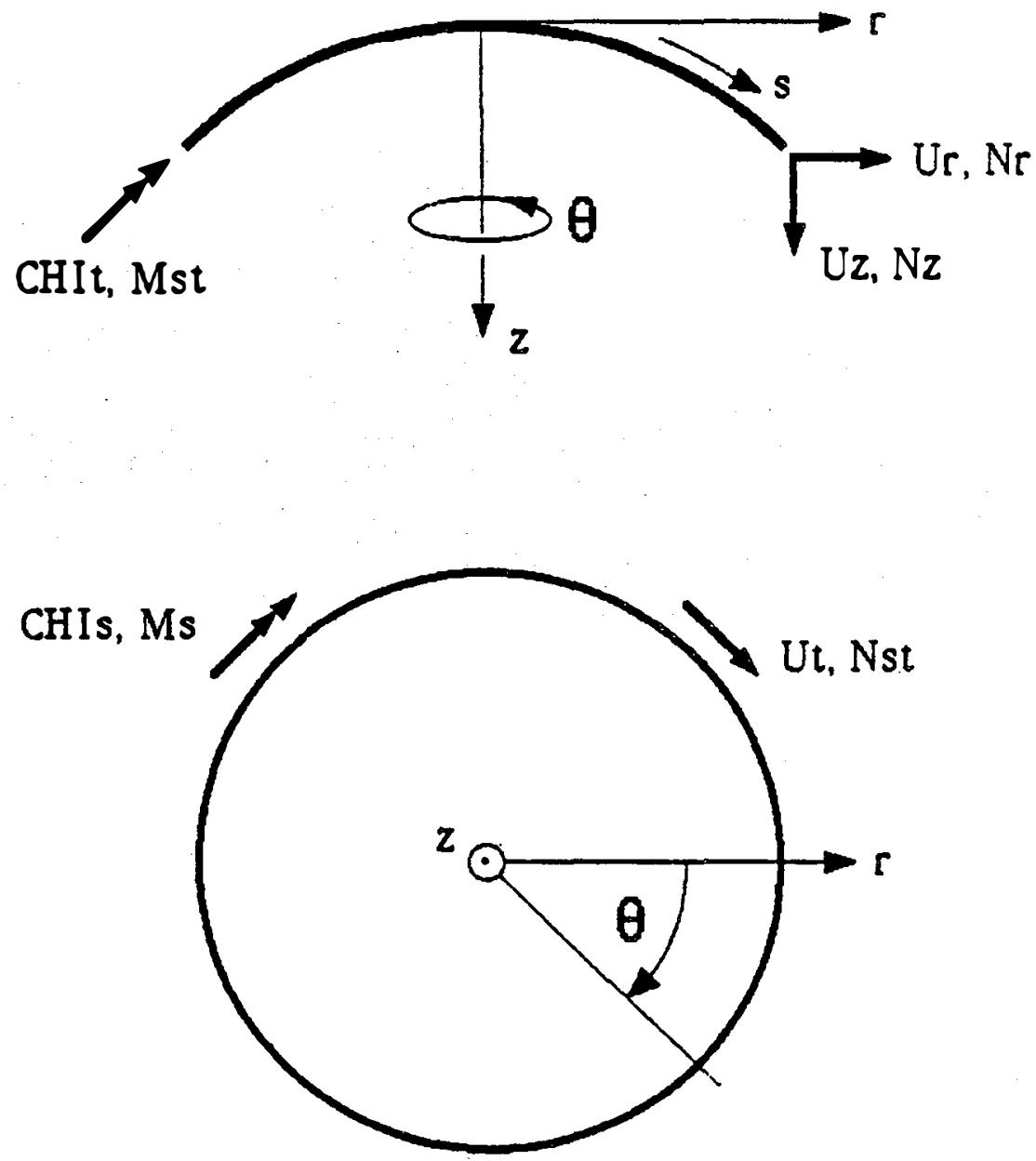

Figure 2: Sign conventions for forces, displacements, moments, and rotations at edge of dish. 


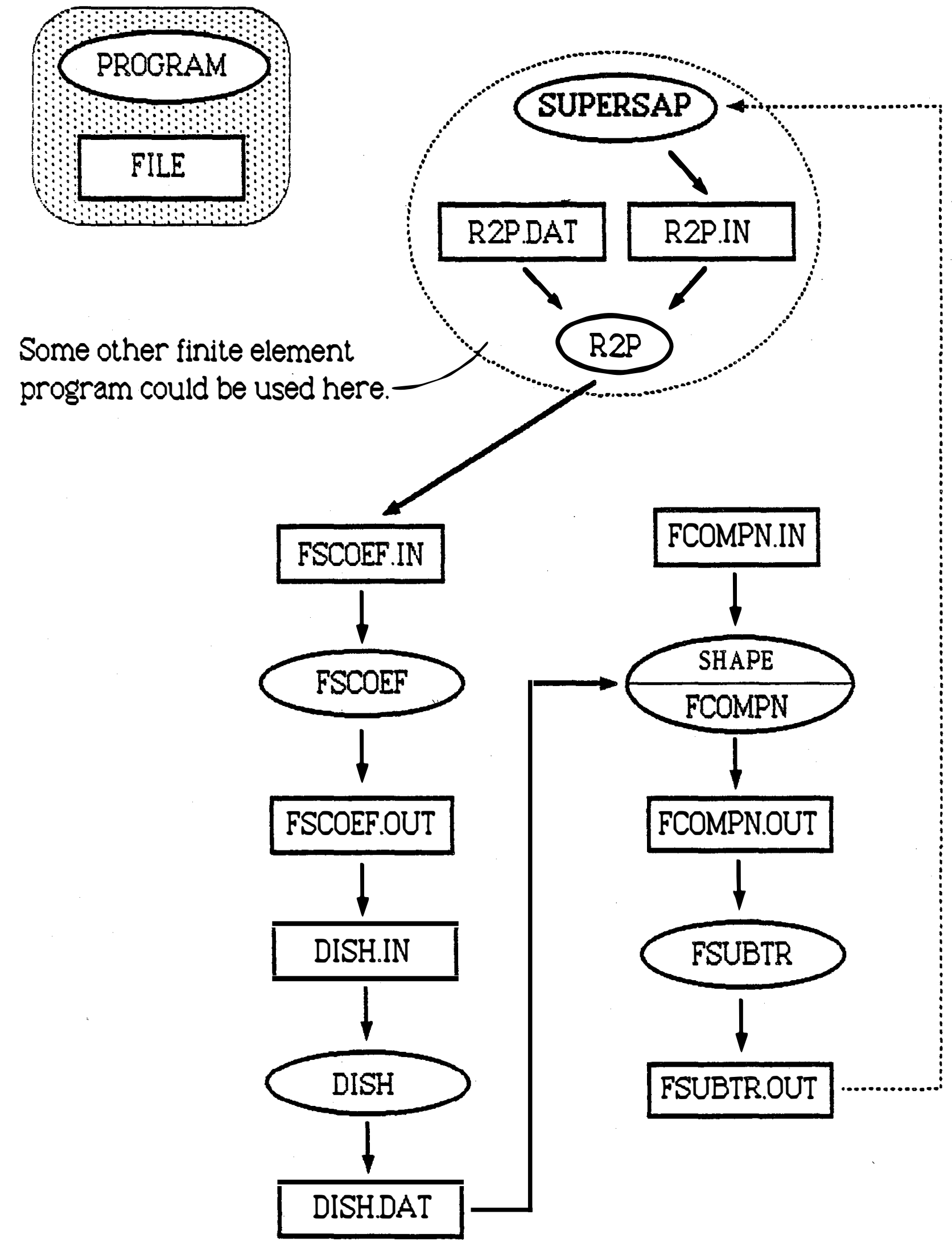

Figure 3: Data path flowchart for enforcing finite element nodal ring displacements in the dish structural analysis. 
Axial Displacement Uz from DAN-KA Run ASDF4E

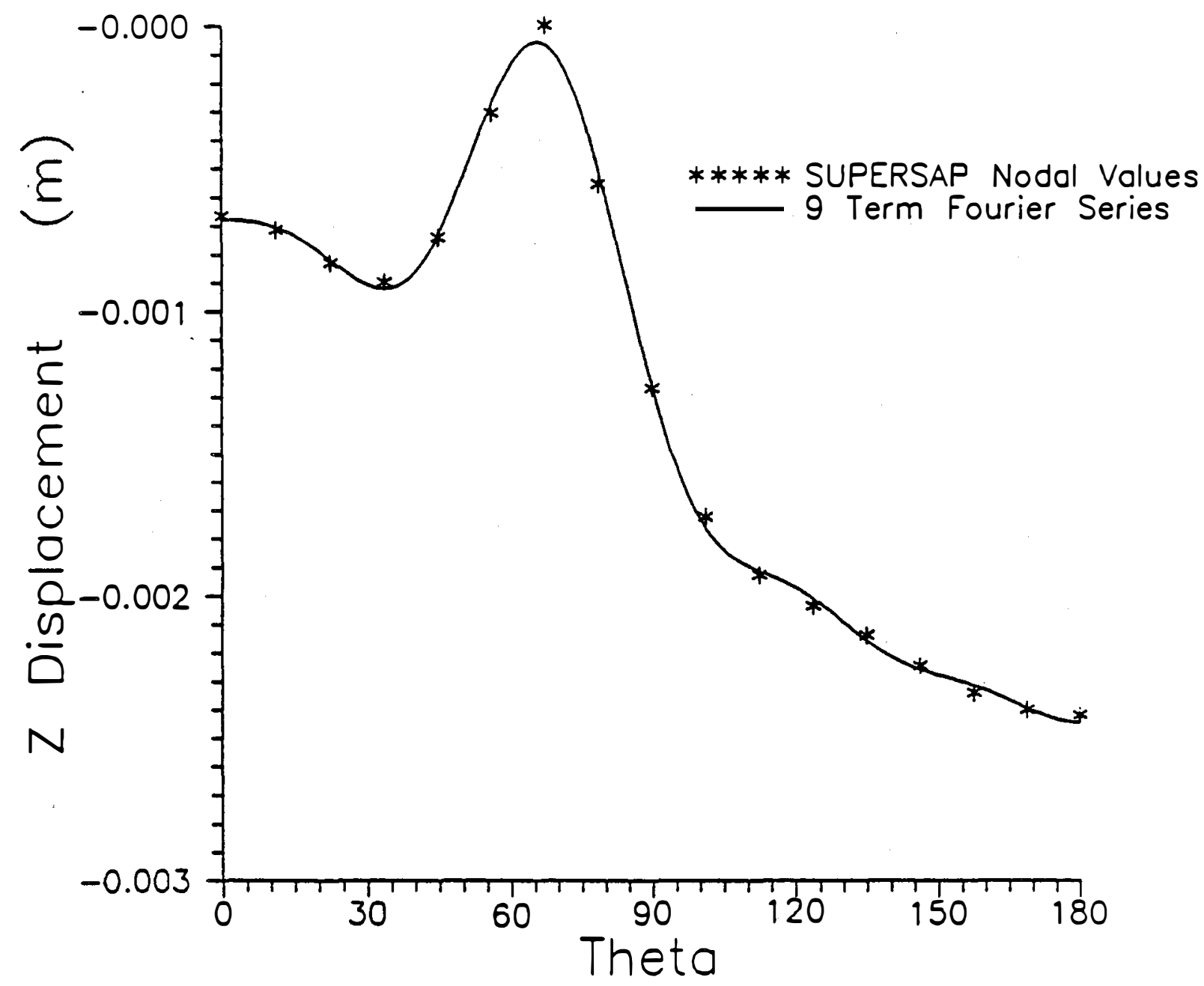

Figure 4: Comparison of SUPERSAP nodal displacements Uz with the nine term Fourier series representation. 


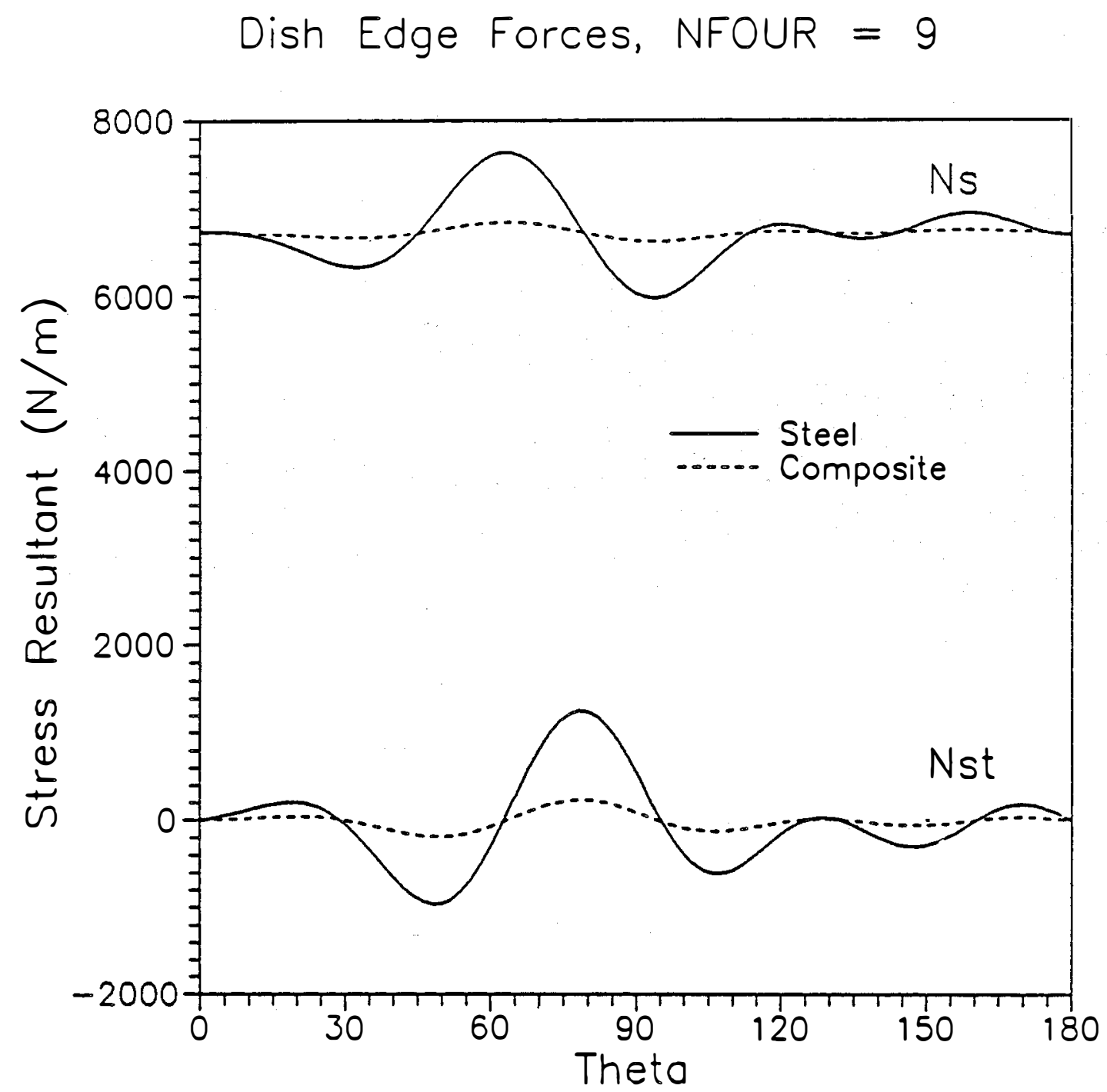

Figure 5: Meridional and tangential stress resultants Ns and Nst due to ring displacements. 
10 mil composite membrane with edge pull, axial transl, and head-on wind load, Dish Radilis= 7.50; Pocal Length $=9.00 ;$ Target Distance= 9.60

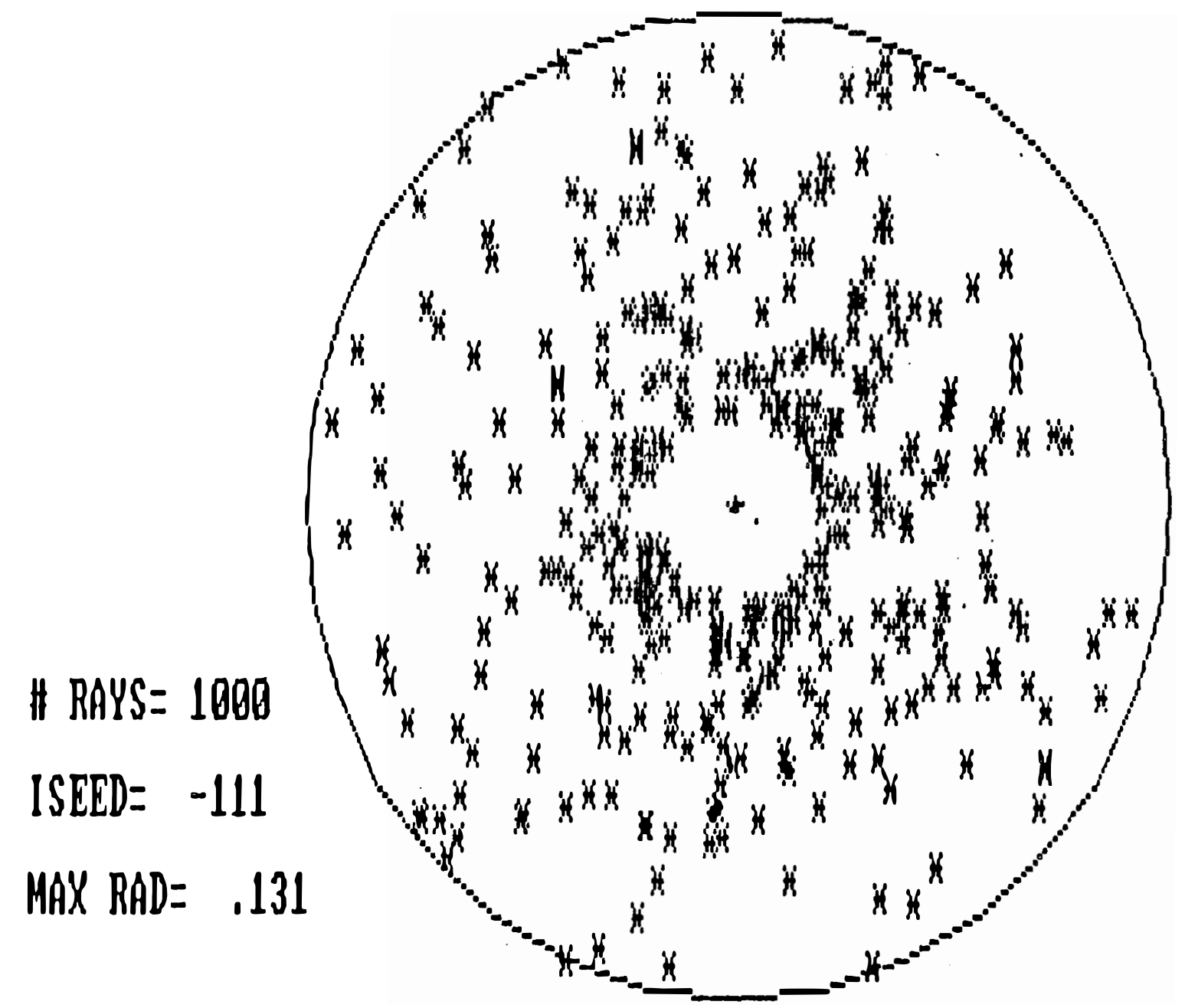
3.60

04.50 6.80

$\times 7.50$ IGRID $=-1$

NR= 0

$N T=0$

RAD $=.131 ;$ RMS RAD = .044; RHS DEV = .034

Figure 6 
18 mil composite membrane with edge pull, axial transl, and wind load, Dish Radius= 7.50; Focal Length= 9,00; Target Distance= 9.00

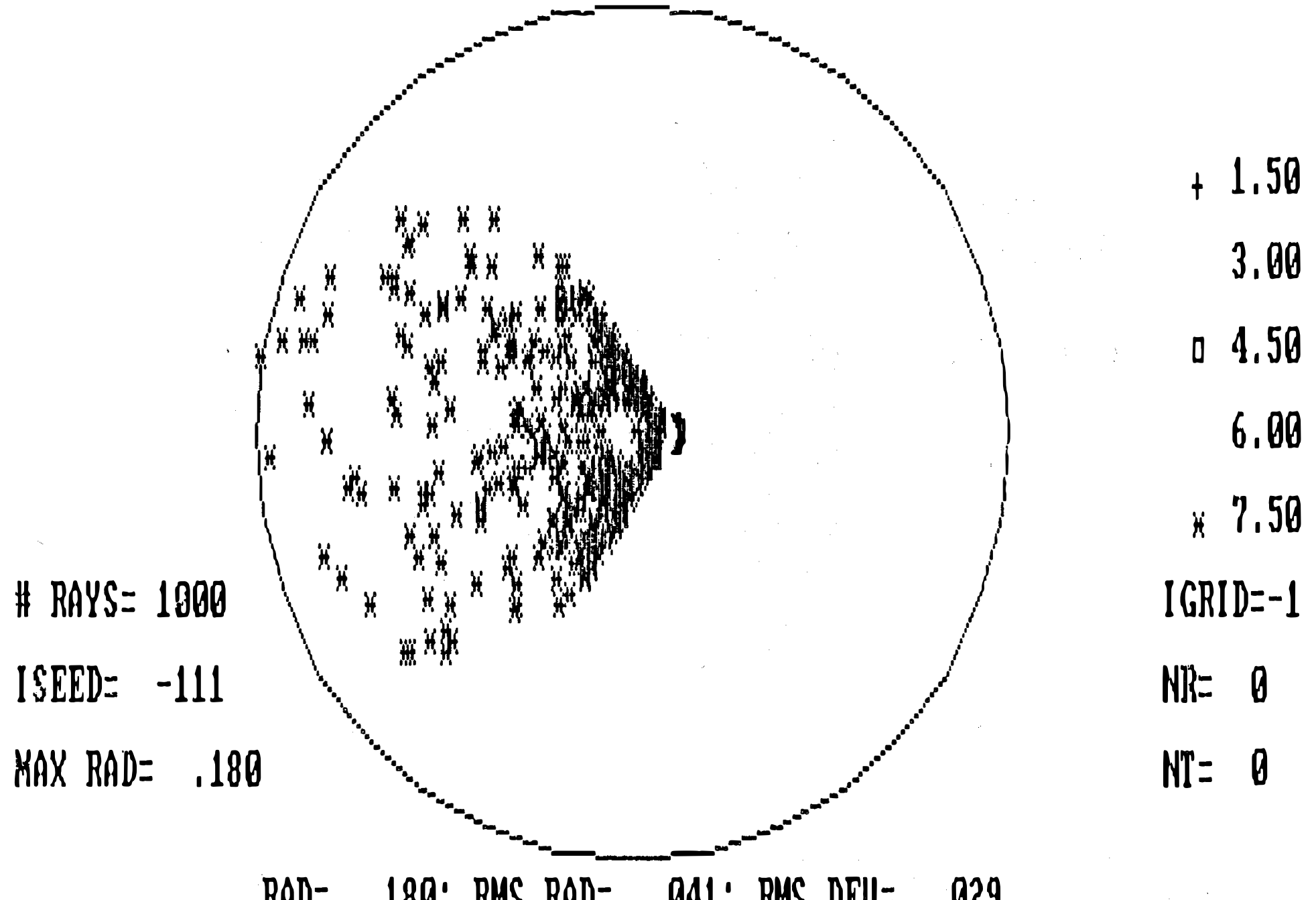

Figure 7 
10 mil composite membrane with edge pull, axial transl, and $1 \mathrm{~cm} n=2$ Ur displ, Dish Radius= 7.50; Focal Length $=9.60 ;$ Target Distance $=9.00$

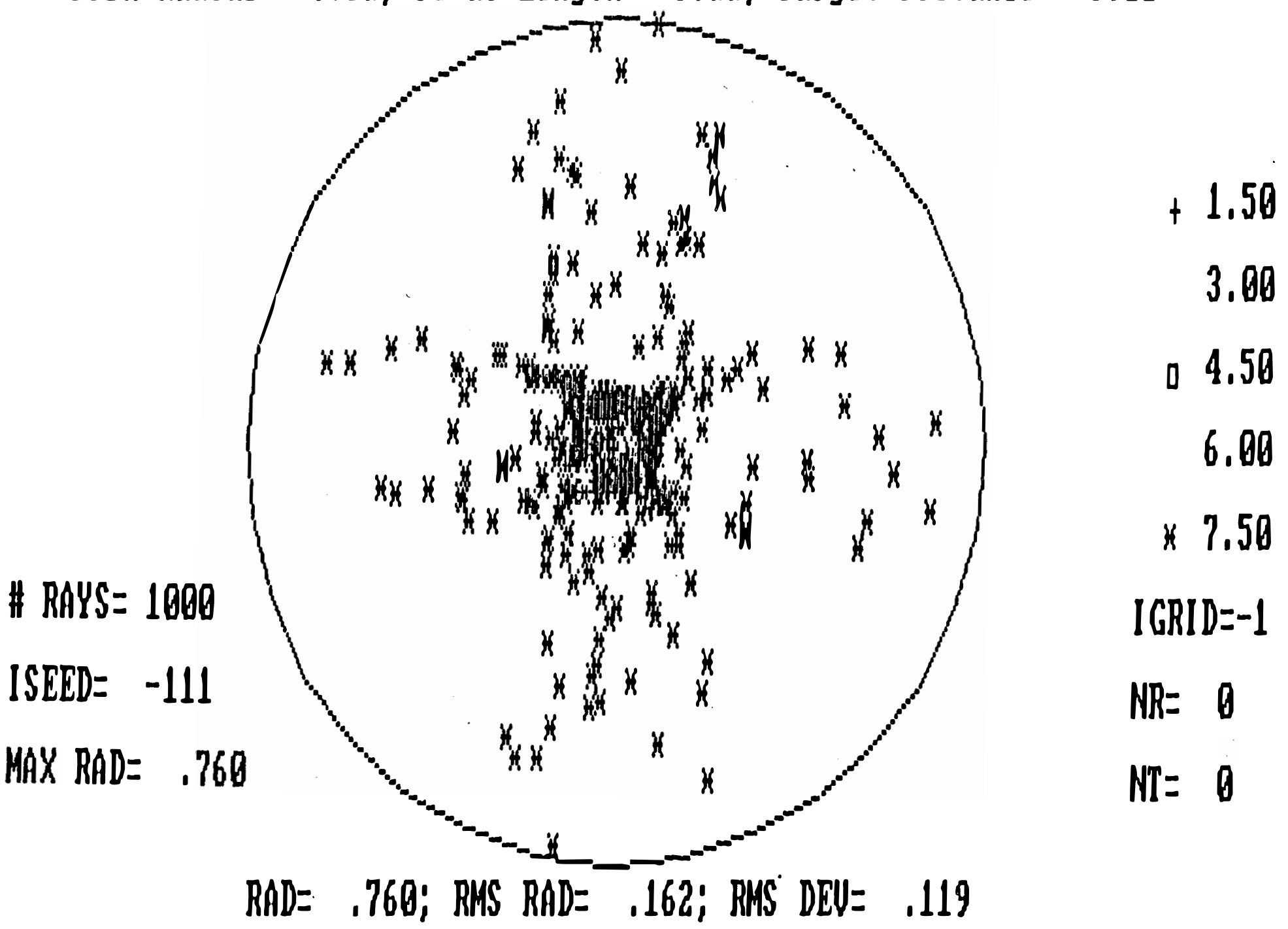

Figure 8 
10 mil compsite membrane; ASDFAE edge displacements, edge pull, axial transl, Dish Radius= 7.50; Focal Length= 9.60; Taryet Distance=9.60

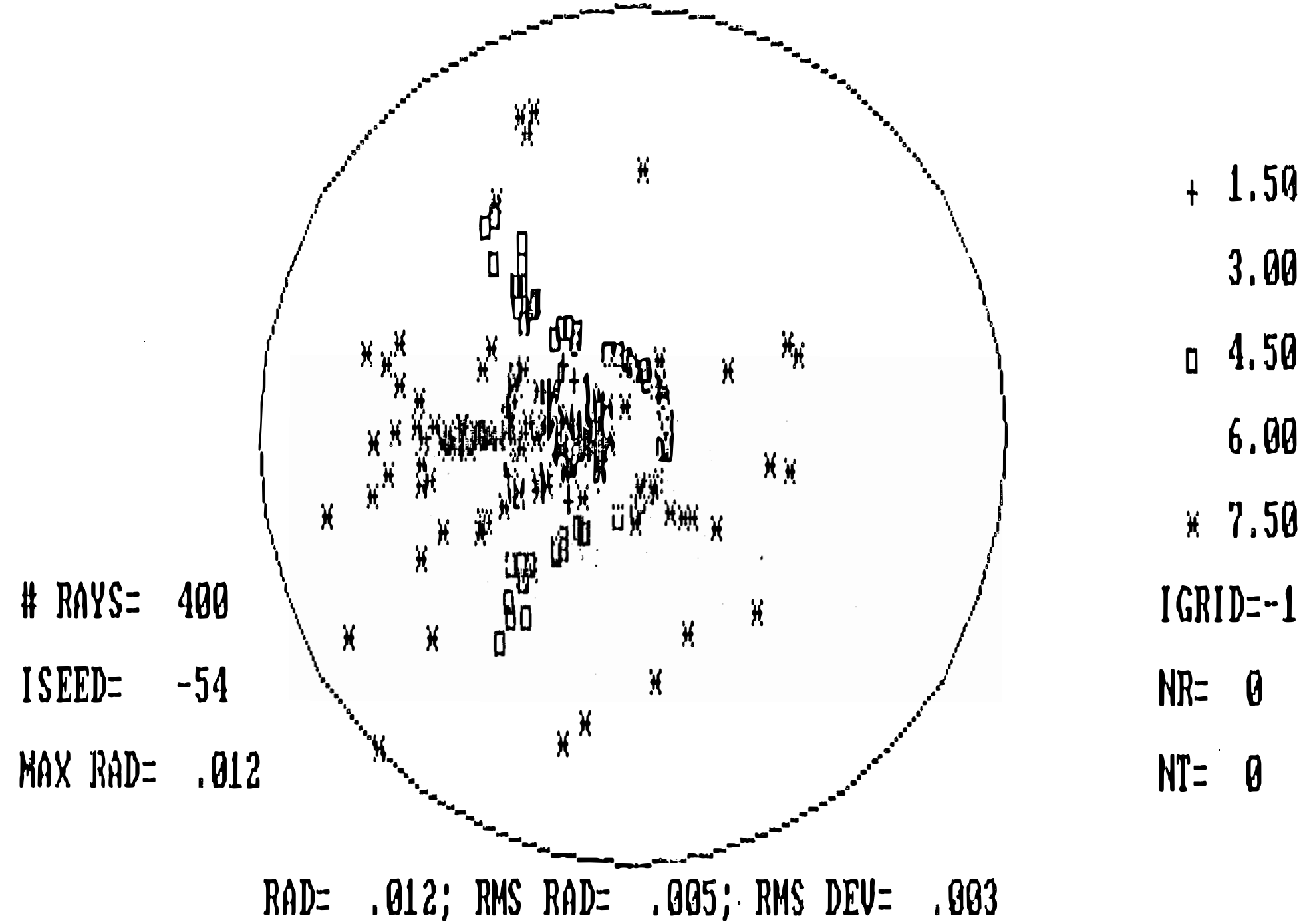

Figure 9 
10 Hil composite membrane: ASDFAE eilge displacements, edge pull, axial transl, Dish Radius= 7.56; Focal Lenyth= 9.00; Dish Surface Location

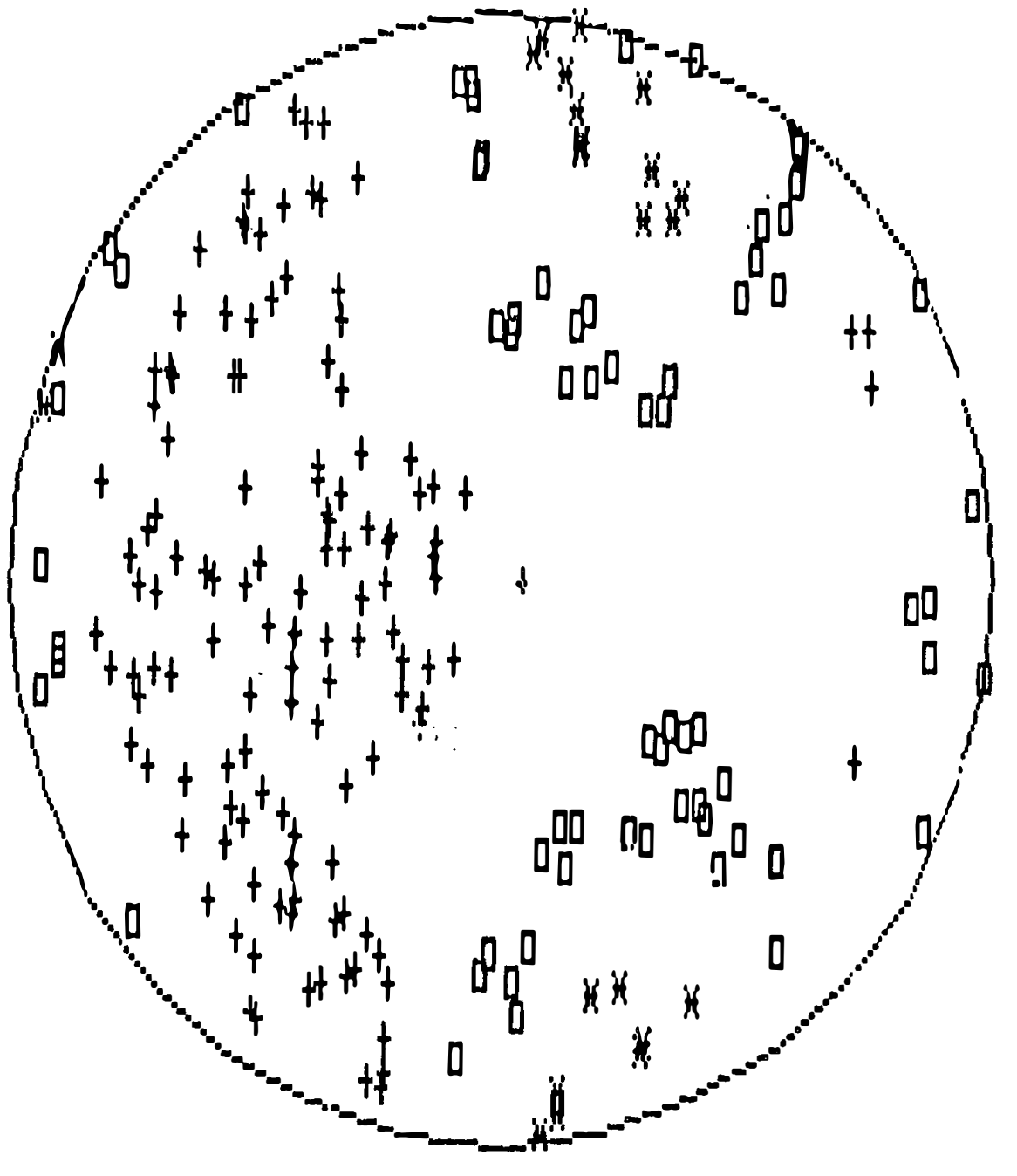

$+.00$

.60

0.01

.01

$* .01$

\# RAYS= 460

ISEED_ 111

IGRID $=-1$

NR= 0

$N T=0$ 


\section{Section 2}

Non-Axisymmetric Optical Model of Solar Dish Concentrators (OPTDSH) 


\section{Non-Axisymmetric Optical Model of Solar Dish Concentrators (OPTDSH)}




\section{Introduction}

A previous program developed at SERI (Kutscher, 1987) used a weighted ray-trace algorithm to provide an optical figure of merit for an axisymmetric reflector having a shape which deviates from an ideal paraboloid of revolution. To study the response of dish concentrators to real-world effects (such as wind and gravity loads, sensitivity to manufacturing tolerances, etc.), nonaxisymmetric structural models (such as finite element simulations or analytical representations like the one described in the main body of this report) are required. In order to quantify the impact of nonaxisymmetric structural deformations, a nonaxisymmetric optical model (which allows calculation of thermal efficiency as well) is also needed. This section documents the development and use of a nonaxisymmetric optical computer program which has been interfaced with the DISH structural code.

General Capabilities of OPTDSH

The present program (OPTDSH) uses a 3-dimensional ray-trace technique as described by Spencer and Murty (1962). Rays are generated at infinity either on a uniform (Cartesian) grid or in a random fashion. Each ray is traced to the dish surface, error terms are incorporated, the resulting direction upon reflection is computed, and the ray is then traced to its intersection with the target plane.

A variety of options have evolved in terms of how the dish surface can be specified. These are schematically indicated in Figure 3-1. A surface can be described by a gridwork of points which give the position (height, $z$ ) of the surface as a function of $(x, y)$ or $(r, \theta)$. Such information is typical output of standard finite element structural codes. The partial derivatives $\delta z / \delta x$ and $\delta z / \delta y$ of the surface at the intersection point of each incoming ray are also needed. OPTDSH could be modified to accept these quantities as input, but presently computes these derivatives and positions between mesh points using a linear 2-dimensional interpolation scheme as outlined by Press et al (1987).

A mesh of points describing a surface can also be analytically generated by a simple user-provided driver as indicated in Figure 1: by the program GENDAT. Two examples of such a program which creates. DAT files appropriate for input to OPTDSH are shown in Figures 2 and 3 . The first line in any of the .DAT input files to OPTDSH is a Title line of textural information. Next, the dish radius, focal length, and target distance (in consistent units) are input. The next line contains a grid flag (IGRID) which tells OPTDSH what type of subsequent data to expect, the number of radial (or X-coordinate) grids (NR), and the number of azimuthal (or $\mathrm{Y}$ coordinate) grids (NT). These parameters are further described in Table 1 . NR radial (or $\mathrm{X}$ ) coordinate values and NT azimuthal (or $Y$ ) coordinate values are then entered as free-formatted input. In the present examples these happen to be on an equally spaced grid, but unequal grids can be used. The remaining information are the positions ( $Z$-coordinate) of the surface at each of the grid points. These are entered in 


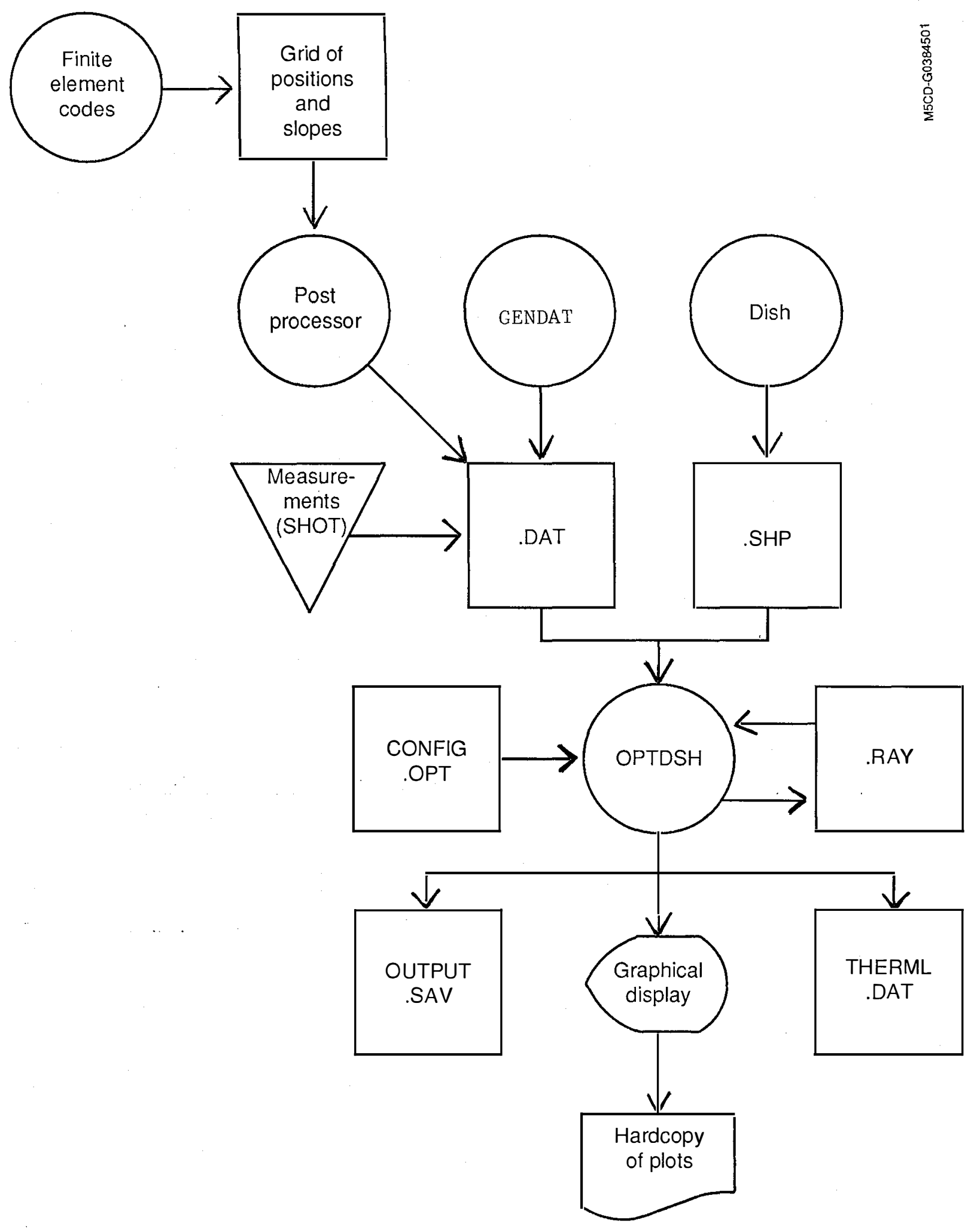

Figure 1. Logic flow and interfaces of OPTDSH 
C: $\backslash$ DISH $-->$ GENDAT

Enter TITLE:

Perfect parabolic dish; $f / D=0.6$; Polar grid

Enter IGRID, NR, NT:

$1,7,5$

Enter dish radius, focal length, and distance to target $(\mathrm{m})$ :

$7.5,9.0,9.0$

Enter name of desired output. DAT file (maximum of 6 characters), prefaced by an optional disk drive designator (default is C:): POLAR

Data File (C:POLAR.DAT) Being Archived .... Stop - Program terminated.

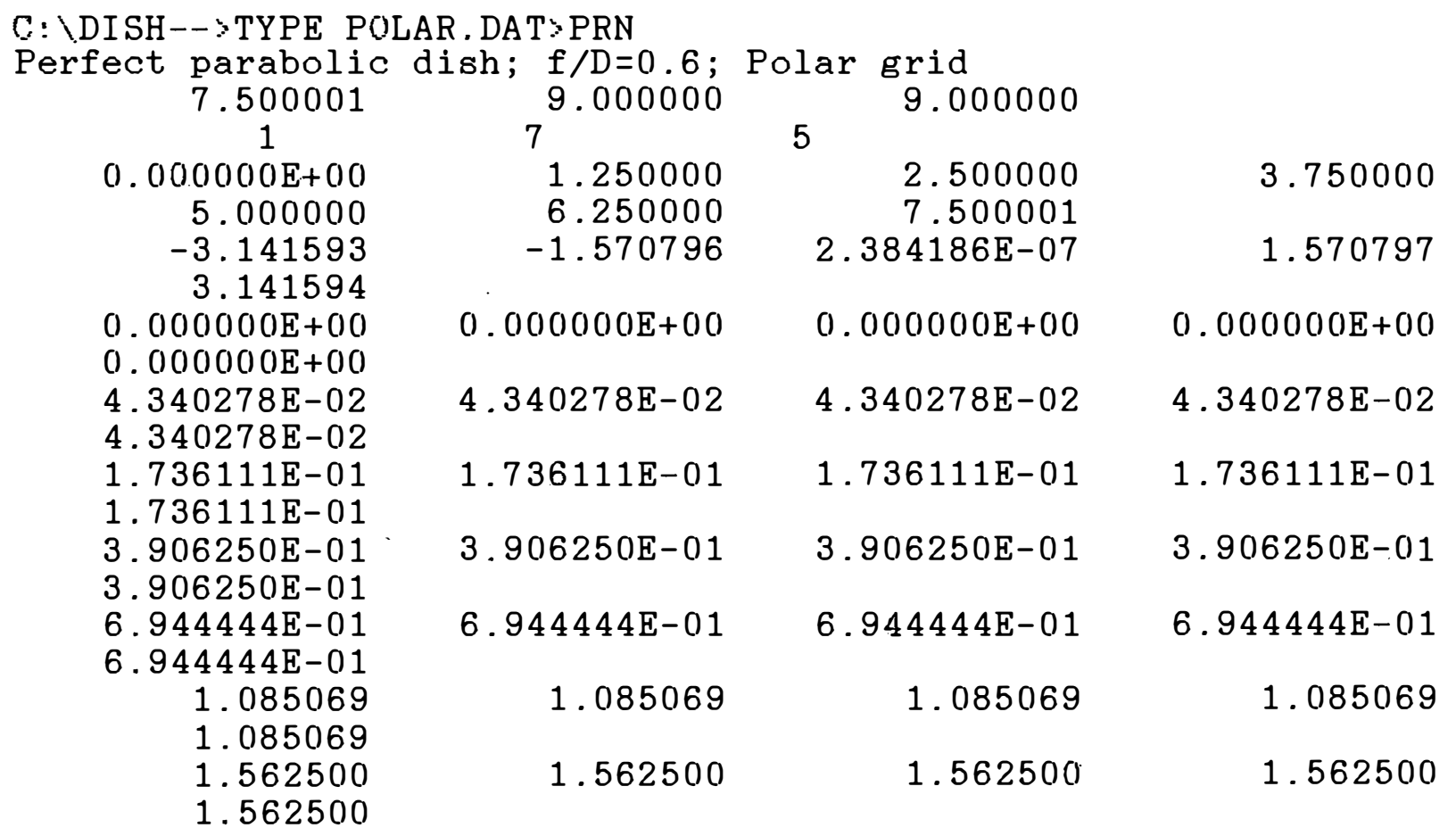
Figure 2. Example GENDAT program which generates .DAT
finite element-like grid points on a polar basis 
C: \DISH-->GENDAT

Enter TITLE:

Perfect parabolic dish; $f / D=0.6$; Cartesian grid

Enter IGRID, NR, NT:

$2,5,5$

Enter dish radius, focal length, and distance to target $(\mathrm{m})$ :

$7.5,9.0,9.0$

Enter name of desired output. DAT file (maximum of 6 characters), prefaced by an optional disk drive designator (default is C:): CARTSN

Data File (C:CARTSN.DAT) Being Archived ....

Stop - Program terminated.

C : \DISH- $>$ TYPE CARTSN.DAT $\gg$ PRN

Perfect parabolic dish; $f / D=0.6$; Cartesian grid

7.500001

2

$-7.500001$

7.500001

$-7.500001$

7.500001

3.125000

3.125000

1. 953125

1.953125

1.562500

1.562500

1.953125

1.953125

3.125000

3.125000
9.000000 5

$-3.750000 \quad 0.000000 E+00$

$-3.750000$

1.953125

$7.812500 \mathrm{E}-01$

$3.906250 \mathrm{E}-01$

$7.812500 \mathrm{E}-01$

1.953125

$0.000000 \mathrm{E}+00$

1.562500

$3.906250 \mathrm{E}-01$

$0.000000 \mathrm{E}+00$

$3.906250 \mathrm{E}-01$

1.562500

3.750000

3.750000

1.953125

$7.812500 \mathrm{E}-01$

$3.906250 \mathrm{E}-01$

$7.812500 \mathrm{E}-01$

1.953125

C : \DISH--

Figure 3. Example GENDAT program which generates .DAT finite element-like grid points on a Cartesian basis 
Table 1. Description of Parameters Contained in Input Files to OPTDSH

\begin{tabular}{|c|c|c|c|c|}
\hline $\begin{array}{l}\text { IGRID/ } \\
\text { ISURF }\end{array}$ & Meaning & NR & NT & $\mathrm{ZA}$ \\
\hline$-1 / 6^{\circ}$ & Use SHAPE & $\begin{array}{r}\text { N/A } \\
(0)\end{array}$ & $\begin{array}{r}\text { N/A } \\
(0)\end{array}$ & $\mathrm{N} / \mathrm{A}$ \\
\hline $0 / 1$ & $\begin{array}{l}\text { Perfect (analytical) } \\
\text { parabola }\end{array}$ & $\begin{array}{r}\mathrm{N} / \mathrm{A} \\
(0)\end{array}$ & $\begin{array}{r}\mathrm{N} / \mathrm{A} \\
(0)\end{array}$ & $\mathrm{N} / \mathrm{A}$ \\
\hline $1 / 4$ & Polar grid of mesh points & $\begin{array}{l}\# R^{\prime} s \\
(>0)\end{array}$ & $\# \underset{(>0)}{\theta \prime}$ & $z-\operatorname{coord}$ \\
\hline $2 / 5$ & $\begin{array}{l}\text { Cartesian grid of mesh } \\
\text { points }\end{array}$ & $\underset{(>0)}{X X^{\prime} s}$ & $\begin{array}{l}\# Y^{\prime} \mathbf{S} \\
(>0)\end{array}$ & $z$-coord \\
\hline $3 / 7$ & $\begin{array}{l}\text { Parabola with line } \\
\text { Gaussians }\end{array}$ & $\begin{array}{c}\text { \# Params } \\
\text { (3) }\end{array}$ & $\begin{array}{c}\# \text { Sets } \\
(14)\end{array}$ & $\begin{array}{l}\text { Param } \\
\text { Values }\end{array}$ \\
\hline $4 / 8$ & $\begin{array}{l}\text { Zernike Monomial } \\
\text { representation }\end{array}$ & $\begin{array}{c}\text { Order of } \\
\qquad \begin{array}{c}\text { fit } \\
(<=4)\end{array}\end{array}$ & $\begin{array}{r}N / A \\
(0)\end{array}$ & $\begin{array}{l}\text { Z-Mono } \\
\text { Coeffs }\end{array}$ \\
\hline
\end{tabular}

$\mathrm{f} / \mathrm{D}=.6$ Parabolic dish represented by Zernike monomials $\begin{array}{cccc}7.500000 & 9.000000 & & 9.000000\end{array}$

0.0

0.0

0.0

0. 0277778

0.0

0.0277778

0.0

0.0

0.0

0.0

Figure 4. Example of .DAT input file which uses zernike monomials 
free-format, NT azimuthal (or Y) values for each of the NR radial (or X) values.

Returning to Figure 1, .DAT information can also be generated by measured surface data. For example, SERI's Scanning Hartmann Optical Tester. (SHOT) (Wood, 1988) allows optical surface measurements to be analyzed in such a way that the surface can be described in terms of a series of Zernike monomials (as discussed by Malacara, 1978). The computed coefficients can then be input to OPTDSH and used to further evaluate optical performance. An example of such an input file is presented as Figure 4.

In Figure 4, the third line of input indicates the grid flag (IGRID=4 for Zernike monomials) and the order of fit to be used (NFIT=3). The number of coefficients, $l$, given by:

$$
\ell=(N F I T+1) *(N F I T+2) / 2
$$

equals 10. The height on the dish surface is then given by:

$$
z(x, y)=\sum_{i=1}^{N F I T+1} \sum_{j=1}^{i} B_{i j} x^{j-1} y^{i-j}
$$

and the partial derivatives are:

$$
\frac{\delta z}{\delta x}=\sum_{i=1}^{N F I T} \sum_{j=1}^{i} j B_{i+1, j+1} x^{j-1} y^{i-j}
$$

and

$$
\frac{\delta z}{\delta y}=\sum_{i=1}^{N F I T} \sum_{j=1}^{i}(i-j+1) B_{i+1, j} x^{j-1} y^{i-j}
$$

with the $\ell$ coefficients given by:

$$
A_{l}=B_{i j}
$$

where:

$$
\ell=[i *(i-1) / 2]+j
$$

In the example in Figure 4 , the fourth and sixth coefficients $(=0.0277778)$ represent the $\mathrm{y}^{2}$ and $\mathrm{x}^{2}$ terms, respectively. All other terms are zero. This set of coefficients, therefore, represents a parabolic surface with a focal length of $\mathrm{f}=9$. 
OPTDSH can also be adapted to study specialized geometries. For example, Figures 5 and 6 show input. DAT files for a perfect parabola $\left(z=r^{2} / 4 f\right)$ and a parabolic dish with a series of line Gaussians (appropriate for analyzing welded seams of various amplitudes and widths) respectively.

In Figure 6, the third line of input (after the title and radius, focal length, and target distance) specifies IGRID=3 (indicating a parabola with line Gaussians), $N R=3$ [number of parameters is 3 : the amplitude ( $A$ ), the $x$-coordinate of each line Gaussian $(\boldsymbol{\mu})$, and the standard deviation $(\sigma)$ associated with each line Gaussian], and the number of line Gaussians is $\mathrm{n}=14$. The next 14 lines contain the amplitude, $\mathrm{x}$-coordinate, and standard deviation of each of the line Gaussians. The surface height is then calculated as:

$$
z(x, y)=(1 / 4 f) *\left(x^{2}+y^{2}\right)+\sum_{i=1}^{n} A_{i} \operatorname{EXP}\left[-1 / 2\left(\left(x-\mu_{i}\right) / \sigma_{i}\right)^{2}\right]
$$

A description of the remaining file interfaces shown in Figure 3-1 will be provided before the interface with DISH is described. A configuration option file (CONFIG.OPT) is read by OPTDSH to define system configutation options. This file also contains default analysis parameters which specify error parameters (sun shape and size, specularity, etc. as outlined by O'Gallagher, 1987) and thermal parameters as required for computing thermal efficiency (as discussed by Lewandowski, 1987).

Output Files

The OUTPUT.SAV file contains histogram frequency information of the number of rays striking concentric annular zones in the target plane. A sample listing of such a file is shown in Figure 7. The first column (RGRID) gives the radius of the annular grid used to tally rays; the maximum radial value used is the radius of the dish. The second column (NBIN) contains the number of rays which intersect the target plane within the corresponding annulus. The next column (FREQ) gives the fraction of the total rays contained within each annulus (NBIN/Total 非 of Rays). The last two columns (TOTRAY and FRACT) present the cumulative number of rays and fraction of rays contained within a given radius respectively.

A THERML.DAT file is also output. This file contains a tabulation of the thermal efficiencies as a function of receiver aperture radius for each operating temperature modeled. An example of such an output file is given in Figure 8 . For each temperature of interest, the annular bin number, the radius of each annular bin, and the number of rays within each bin are tabulated in the first three columns. The last two columns give the thermal efficiencies for a collector with a primary only and for a primary with secondary concentrator. Such efficiencies are calculated as indicated by Lewandowski (1987). 
$\begin{array}{ccc}\text { Perfect } & \text { (analytical) } & \text { parabolic dish; } \\ 7.500000 & 9.000000 & 0.6 \\ 0 & 0 & 9.000000\end{array}$

Figure 5. Example of .DAT input file for analytic surface

$\mathrm{f} / \mathrm{D}=.6$ Parabolic dish with line Gaussians; $A=5 \mathrm{~mm} ; 1 \mathrm{~m}$ wide; $\mathrm{s}=5 \mathrm{~mm}$ 7.500000 3

$.005,-6.5, .005$

9.000000 14

$.005,-5.5, .005$

$.005,-4.5, .005$

$.005,-3.5, .005$

$.005,-2.5, .005$

$.005,-1.5, .005$

$.005,-0.5, .005$

$.005, \quad 0.5, .005$

$.005,1.5, .005$

$.005,2.5, .005$

$.005, \quad 3.5, .005$

$.005,4.5, .005$

$.005,5.5, .005$

$.005,6.5, .005$

Figure 6. Example of .DAT input file for modeling welded seams 
Focal Length $=$
Number of Rays $=500$

Seed for Ray Generation =

Dish Radius $=7.500$

$-5$

Distance to Target $=9.000$

Delta X,Y $=.0000$

Maximum Radial Intersection of Target Plane =

RMS Radius of Distribution on Target Plane =

RMS Deviation from Mean =

$\begin{array}{rrr}\text { RGRID } & \text { NBIN } & \text { FREQ } \\ .07500 & 442 & .88400\end{array}$

. 05010

.22500

$56 \quad .11200$

.37500

.00400

.52500

.00000

.67500

.00000

.82500

.00000

.97500

.00000

1. 12500

.00000

1. 27500

.00000

1. 42500

.00000

1.57500

.00000

1.72500

.00000

1.87500

.00000

2. 02500

.00000

2. 17500

.00000

2. 32500

.00000

.00000

2.47500

.00000

2.62500

.00000

2.92500

.00000

3.07500

.00000

.00000

.00000

.00000

.00000

.00000

.00000

3.97500

.00000

4. 12500

.00000

4.27500

.00000

.00000

TOTRAY

$482 . \quad .88400$

$498 . \quad .99600$

$500 . \quad 1.00000$

500.1 .00000

500.1 .00000

500.1 .00000

500.1 .00000

500.1 .00000

500.1 .00000

$500 . \quad 1.00000$

500.1 .00000

500.1 .00000

500.1 .00000

500.1 .00000

500.1 .00000

$500 . \quad 1.00000$

500.1 .00000

500.1 .00000

500.1 .00000

$500 . \quad 1.00000$

500.1 .00000

500.1 .00000

500.1 .00000

500.1 .00000

500.1 .00000

500.1 .00000

$500 . \quad 1.00000$

$500 . \quad 1.00000$

$500 . \quad 1.00000$

500.1 .00000

4.57500

.00000

500.1 .00000

4.72500

.00000

500.1 .00000

4.87500

.00000

$500 . \quad 1.00000$

500.1 .00000

5.17500

.00000

500

1.00000

.00000

500.1 .00000

.00000

500.1 .00000

.00000

500.1 .00000

.00000

500.1 .00000

500.1 .00000

500.1 .00000

$500 . \quad 1.00000$

$500 . \quad 1.00000$

$\begin{array}{ll}500 . & 1.00000 \\ 500 . & 1.00000\end{array}$

.31784

.10347

5.92500

00000

6.22500

.00000

.00000

.00000

500.1 .00000

500.1 .00000

.00000

500.1 .00000

.00000

500.1 .00000

Figure 7. Sample

OOTPUT.SAV file

6.82500

.00000

500.1 .00000 


\begin{tabular}{|c|c|c|c|c|c|}
\hline \multicolumn{2}{|c|}{ Temperature $=500.0000$ degrees $\mathrm{C}$} & $\begin{array}{l}500.0000 \\
(\mathrm{~J}), \text { ETAVSH }\end{array}$ & $\begin{array}{l}\text { es C } \\
\text {, I ), EI }\end{array}$ & $(\mathrm{J}, 2, \mathrm{I}$ & \\
\hline 1 & .0000 & 8.0000 & .0000 & .0000 & \\
\hline 2 & .0059 & 19.0000 & .0034 & .0023 & \\
\hline 3 & .0117 & 41.0000 & .0114 & .0101 & \\
\hline 4 & .0176 & 44.0000 & .0286 & .0261 & \\
\hline 5 & .0235 & 59.0000 & .0471 & .0433 & \\
\hline 6 & .0294 & 70.0000 & .0719 & .0668 & \\
\hline 7 & .0352 & 76.0000 & .1013 & .0946 & \\
\hline 8 & .0411 & 85.0000 & .1332 & .1248 & \\
\hline 9 & .0470 & 101.0000 & .1689 & .1587 & \\
\hline 10 & .0529 & 86.0000 & .2113 & .1990 & \\
\hline 11 & .0587 & 100.0000 & .2473 & .2337 & \\
\hline 12 & .0646 & 89.0000 & .2892 & .2739 & \\
\hline 13 & .0705 & 94.0000 & .3264 & .3101 & \\
\hline 14 & .0764 & 90.0000 & .3656 & .3487 & \\
\hline 15 & .0822 & 90.0000 & .4031 & .3855 & \\
\hline 16 & .0881 & 95.0000 & .4406 & .4221 & \\
\hline 17 & .0940 & 84.0000 & .4801 & .4610 & \\
\hline 18 & .0999 & 85.0000 & .5149 & .4954 & \\
\hline 19 & .1057 & 70.0000 & .5500 & .5302 & \\
\hline 20 & .1116 & 88.0000 & .5788 & .5593 & \\
\hline 21 & .1175 & 66.0000 & .6151 & .5951 & \\
\hline 22 & .1234 & 64.0000 & .6421 & .6226 & \\
\hline 23 & .1292 & 40.0000 & .6681 & .6493 & \\
\hline 24 & .1351 & 45.0000 & .6840 & .6671 & \\
\hline 25 & .1410 & 53.0000 & .7019 & .6863 & \\
\hline 26 & .1469 & 43.0000 & .7231 & .7086 & \\
\hline 27 & .1527 & 33.0000 & .7400 & .7268 & \\
\hline 28 & .1586 & 26.0000 & .7527 & .7410 & \\
\hline 29 & .1645 & 17.0000 & .7623 & .7530 & \\
\hline 30 & .1704 & 21.0000 & .7681 & .7612 & \\
\hline 31 & .1762 & 19.0000 & .7755 & .7706 & \\
\hline 32 & .1821 & 23.0000 & .7820 & .7791 & \\
\hline 33 & .1880 & 12.0000 & .7902 & .7884 & \\
\hline 34 & .1939 & 11.0000 & .7936 & .7935 & \\
\hline 35 & .1997 & 10.0000 & .7966 & .7983 & \\
\hline 36 & .2056 & 11.0000 & .7991 & .8029 & \\
\hline 37 & .2115 & 4.0000 & .8019 & .8076 & \\
\hline 38 & .2174 & 9.0000 & .8018 & .80194 & \\
\hline 39 & .2232 & 3.0000 & .8037 & .8129 & \\
\hline 40 & .2291 & 4.0000 & .8030 & .8138 & \\
\hline 41 & .2350 & 2.0000 & .8027 & .8149 & \\
\hline 42 & .2409 & 2.0000 & .8015 & .8151 & \\
\hline 43 & .2467 & 1.0000 & .8002 & .8155 & \\
\hline 44 & .2526 & 3.0000 & .7985 & .8153 & \\
\hline 45 & .2585 & 1.0000 & .7975 & .8160 & Figure 8. Sample \\
\hline 46 & .2644 & .0000 & .7957 & .8158 & TAERML.DAT file \\
\hline 47 & .2702 & .0000 & .7934 & .8149 & \\
\hline 48 & .2761 & .0000 & .7910 & .8140 & \\
\hline 49 & .2820 & 1.0000 & .7886 & .8130 & \\
\hline 50 & .2878 & .0000 & .7865 & .8124 & \\
\hline 51 & .2937 & .0000 & .7840 & .8114 & \\
\hline
\end{tabular}




\begin{tabular}{|c|c|c|c|c|c|}
\hline $\begin{array}{r}\text { Temp } \\
\mathrm{J}, \mathrm{F}\end{array}$ & $\begin{array}{l}\bar{e} \\
, \text { DIST }\end{array}$ & (J), ETAVSF & $\begin{array}{l}\text { es C } \\
\text {, I ), EI }\end{array}$ & $(J, 2, I):$ & \\
\hline 1 & .0000 & 8.0000 & .0000 & .0000 & \\
\hline 2 & .0059 & 19.0000 & .0033 & .0022 & \\
\hline 3 & .0117 & 41.0000 & .0111 & .0099 & \\
\hline 4 & .0176 & 44.0000 & .0282 & .0259 & \\
\hline 5 & .0235 & 59.0000 & .0463 & .0429 & \\
\hline 6 & .0294 & 70.0000 & .0706 & .0661 & \\
\hline 7 & 0352 & 76.0000 & 0995 & .0936 & \\
\hline 8 & .0411 & 85.0000 & .1307 & .1234 & \\
\hline 9 & .0470 & 101.0000 & .1657 & .1569 & \\
\hline 10 & .0529 & 86.0000 & .2072 & .1967 & \\
\hline 11 & .0587 & 100.0000 & .2422 & .2309 & \\
\hline 12 & .0646 & 89.0000 & .2831 & .2705 & \\
\hline 13 & .0705 & 94.0000 & .3191 & .3061 & \\
\hline 14 & .0764 & 90.0000 & .3570 & .3440 & \\
\hline 15 & .0822 & 90.0000 & .3931 & .3800 & \\
\hline 16 & .0881 & 95.0000 & .4291 & .4159 & \\
\hline 17 & .0940 & 84.0000 & .4670 & .4539 & \\
\hline 18 & 0999 & 85.0000 & .5002 & .4874 & \\
\hline 19 & .1057 & 70.0000 & .5336 & .5212 & \\
\hline 20 & .1116 & 88.0000 & .5604 & .5493 & \\
\hline 21 & .1175 & 66.0000 & .5948 & .5840 & \\
\hline 22 & .1234 & 64.0000 & .6197 & .6103 & \\
\hline 23 & .1292 & 40.0000 & .6435 & .6358 & \\
\hline 24 & .1351 & 45.0000 & .6571 & .6523 & \\
\hline 25 & .1410 & 53.0000 & .6726 & .6702 & \\
\hline 26 & .1469 & 43.0000 & .6913 & .6911 & \\
\hline 27 & .1527 & 33.0000 & .7057 & .7080 & \\
\hline 28 & .1586 & 26.0000 & .7156 & .7207 & \\
\hline 29 & .1645 & 17.0000 & .7224 & .7312 & \\
\hline 30 & .1704 & 21.0000 & .7253 & .7378 & \\
\hline 31 & .1762 & 19.0000 & .7297 & .7455 & \\
\hline 32 & .1821 & 23.0000 & .7331 & .7523 & \\
\hline 33 & .1880 & 12.0000 & .7381 & .7598 & \\
\hline 34 & . 1939 & 11.0000 & .7382 & .7631 & \\
\hline 35 & 1997 & 10.0000 & .7378 & .7661 & \\
\hline 36 & .2056 & 11.0000 & .7367 & .7688 & \\
\hline 37 & .2115 & 4.0000 & .7360 & .7715 & \\
\hline 38 & .2174 & 9.0000 & .7321 & .7713 & \\
\hline 39 & .2232 & 3.0000 & .7302 & .7726 & \\
\hline 40 & .2291 & 4.0000 & .7256 & .7714 & \\
\hline 41 & .2350 & 2.0000 & .7213 & .7703 & \\
\hline 42 & .2409 & 2.0000 & .7159 & .7683 & \\
\hline 43 & .2467 & 1.0000 & .7104 & .7663 & \\
\hline 44 & .2526 & 3.0000 & .7044 & .7638 & \\
\hline 45 & .2585 & 1.0000 & .6990 & .7620 & \\
\hline 46 & .2644 & .0000 & .6926 & .7593 & Figure 8 (continued). Sample \\
\hline 47 & .2702 & .0000 & .6857 & .7559 & \\
\hline 48 & .2761 & .0000 & .6786 & .7524 & \\
\hline 49 & .2820 & 1.0000 & .6714 & .7488 & \\
\hline 50 & .2878 & .0000 & .6644 & .7455 & \\
\hline 51 & .2937 & .0000 & .6568 & .7417 & \\
\hline
\end{tabular}


The capability of archiving and re-reading a file which contains the intersection points of each ray with both the dish surface and the target plane has been incorporated. The ray information is saved as a binary file to reduce storage requirements and access time. Roughly 16-19 bytes per ray (depending on the number of rays traced) are required to archive the data.

Menu Options

An overview of the various menu options which allow the user to access the capabilities of OPTDSH are shown in Figure 9. The first set of options allows modification of configuration parameters, ànalyses to be performed, or termination of the program. Desired options are specified via Function-Key entry as shown in Figure 10. Return to the previous menu level can also be achieved by pressing the Escape key. The main menu is initially obtained by running the OPTDSH program by entering:

\section{OPTDSH<Enter>}

Configuration

Selection of the Configuration option (F1) allows access to the system, error, and thermal analysis parameters as shown in Figure 11. The various specification options associated with each of these parameters are illustrated in Figures 12 thru 14, respectively. To change a given parameter, enter parameter(s) and their desired (allowable) value(s) as "Parameter"="Value". Multiple changes can be entered at a single time. For example in Figure 12, to change the default disk drive designation from $C$ to $D$ and to change the graphics board from EGA to CGA type:

IDRV=D, IGRF=2<Enter $>$

When all parameters of a given category have been suitably altered a blank line (<Enter> only) returns to the menu shown in Figure 11. Modifications to default parameters can be updated to the CONFIG.OPT file (so that these new options will be used each time OPTDSH is executed) by pressing the F2 key, or modified parameters can be used locally for the present analysis only by pressing the Fl key.

Analysis

Once the desired configuration parameters have been set (or the initial default values are desired to be used), the analysis module of OPTDSH can be accessed via the F2 key from the main menu (Figure 10). The user will then be prompted for the type of input file desired (Figure 15) as discussed above under "General Capabilities". Once a file type has been selected, the user must provide a file name to be used. The present example (Figure 15) uses a file named PARAB.DAT located on the C: drive. To indicate this enter:

PARAB $<$ Enter $>$ 


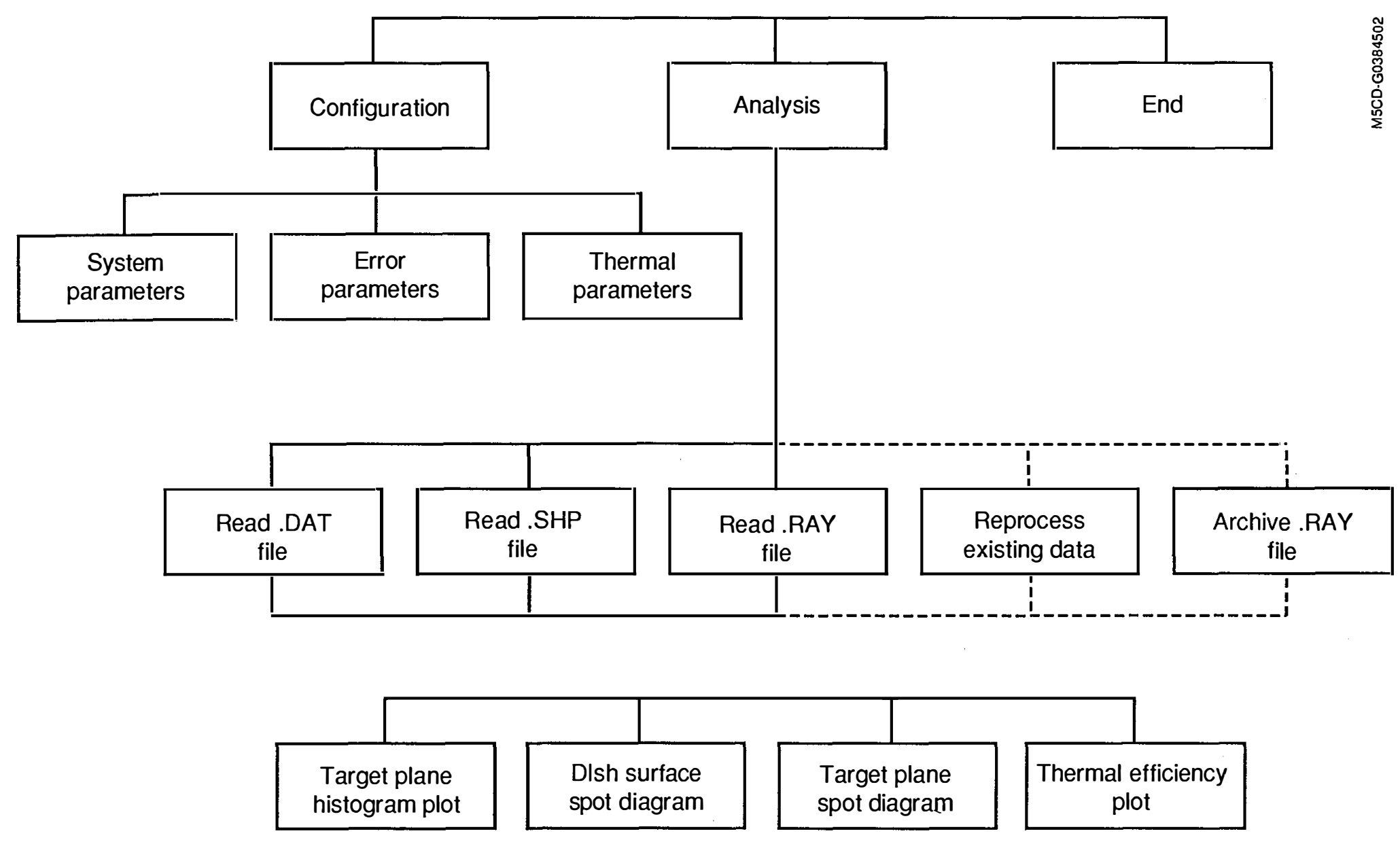

Figure 9. Menu options of OPTDSB 
Select Option:

F1: Configuration

E2: Analysis

F 3: End

Enter F-Key Choice: Figure 10. Example of main menu options

Select Configuration Option:

End Configuration:

F1: Do NOT Update Configuration File

F2: DO Update Configuration File

Modify Parameters:

F 3: System

F 4: Error

F5: Thermal

Enter F-Key Choice: Figure 11. Example of configuration option menu

\section{SYSTEM PARAMETERS}

$\begin{array}{cc}\text { Parameter } & \text { Value } \\ \text { ISYS } & 3 \\ \text { IDRV } & \mathrm{C} \\ \text { IPRT } & 2 \\ \text { IGRE } & 3\end{array}$

Description

SYStem type; 1=PC, 2=XT, 3=AT, 4=Compac default disk DRiVe designation; $A-F$

PRinTer type; $0=$ None, 2=Epson

GRaPHics board; 2=CGA, 3=EGA

Enter Parameter(s) \& Value(s) to be changed (separated by commas) as Parameter = Value; Null Entry/Carriage Return indicates no further changes:

Figure 12. Example of system parameter specification options 


\section{ERROR PARAMETERS}

Parameter

SLOP

SPEC

SUNS

TRAK

DIST

$$
\begin{aligned}
& \text { Value } \\
& 3.000 \\
& 1.500 \\
& 2.730 \\
& .000 \\
& 1.000
\end{aligned}
$$

Description
SLOPe error (mrad); $>=0.0$
SPECularity error (mrad); $>=0.0$
SUN Sigma (mrad); $>=0.0$
TRACKer error (mrad); $>=0.0$
error DISTribution; $0=$ None, $1=$ Gaussian, $2=$ Pillbox

Enter Parameter(s) \& Value(s) to be changed (separated by commas) as Parameter = Value; Null Entry/Carriage Return indicates no further changes:

\section{Figure 13. Example of error parameter specification options}

$\begin{array}{crl}\text { Parameter } & \text { Value } & \text { Description } \\ \text { TAMB } & 20.000 & \text { Ambient Temperature (deg. C) } \\ \text { TRC1 } & 500.000 & \text { ReCeiver Temperature for analysis \#1 (deg. C) } \\ \text { TRC2 } & 800.000 & \text { ReCeiver Temperature for analysis \#2 (deg. C) } \\ \text { TRC3 } & .000 & \text { ReCeiver Temperature for analysis \#3 (deg. C) } \\ \text { TRC4 } & .000 & \text { ReCeiver Temperature for analysis \#4 (deg. C) } \\ \text { NTMP } & 2.000 & \text { Number of TeMPerature analyses to be run (0-4) } \\ \text { ABSR } & .982 & \text { effective receiver ABSoRptance (0-1) } \\ \text { EMIT } & .998 & \text { effective receiver EMITtance (0-1) } \\ \text { ARAT } & 5.000 & \text { Area RATio of receiver wall to aperture } \\ \text { RSCL } & 1.000 & \text { Receiver aperture SCaLe factor } \\ \text { NBIN } & 50.000 & \text { Number of radial BINs for thermal analysis } \\ \text { RHO1 } & .900 & \text { reflectance of primary mirror } \\ \text { RHO2 } & .900 & \text { reflectance of secondary mirror } \\ \text { BLOK } & .960 & \text { BLOcKage factor (0-1) } \\ \text { DNIR } & 800.000 & \text { Direct Normal IRradiance (W/m^2) } \\ \text { COND } & .737 & \text { CONDuctive heat loss coefficient (W/m²-K) } \\ \text { DELE } & .000 & \text { DELta Focal length distance (m) }\end{array}$

Enter Parameter(s) \& Value(s) to be changed (separated by commas) as Parameter = Value; Null Entry/Carriage Return indicates no further changes:

Figure 14. Example of thermal analysis parameter specification options 
Select Data File Option:

F1: Finite Element Data (.DAT)

F2: Shape Data (.SHP)

F3: Ray Data (.RAY)

F 4: Return to Previous Menu

Enter F-Key Choice:

Enter name of desired. DAT input file (maximum of 6 characters), prefaced by an optional disk drive designator (default is C:):

PARAB

Data File (C:PARAB.DAT ) Being Read ....

Enter (Approximate) \# of Rays (1-10000) to be Traced: 2000

Enter Integer Seed for Ray Generation between \pm 32767 345 ( $0=$ uniform X-Y Grid) :

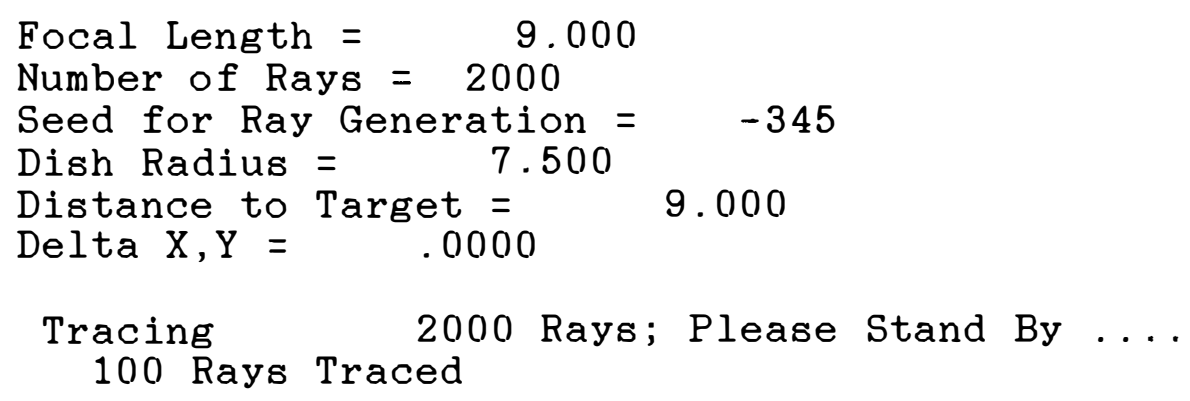

Figure 15. Example of analysis session using OPTDSB

Next, the number of rays desired to be traced must be specified (the present maximum is 10,000$)$. Enter:

$$
\text { \# of Rays<Enter> }
$$

Finally, an integer seed for ray generation and error term treatment is required. Any number between -32767 and +32767 can be used (note that positive numbers will automatically be converted to negative numbers for purposes of random number generation). A seed of zero ( 0 ) will cause a uniform X-Y grid of rays to be used rather than a randomly generated pattern. Enter:

Seed for Random Number Generation<Enter>

Some information about the specified configuration being traced is echoed to the computer monitor and the number of rays traced is updated after every 100 rays. 
Select Graphics Option:

F1: Histogram of Radial Distance in Target Plane
F2: Spot Diagram of Dish Surface
F3: Spot Diagram in Target Plane
F 4: Thermal Efficiency Plot
F5: Return to Previous Menu
Enter F-Key Choice:

Figure 16. Example of graphical display options menu

Graphical Display

After the specified number of rays have been traced, the graphical display options menu is presented (Figure 16). It should be noted that OPTDSH uses a graphics subroutine library named Grafmatics (1984); prospective users wishing to re-compile the OPTDSH source code must have access to the appropriate version (in terms of FORTRAN compiler and system graphics hardware) of this commercial product.

The first graphics option (F1) generates a histogram plot of the number of rays contained within concentric annuli in the target plane. This is simply a plot of the RVALS(J) and DIST $(\mathrm{J})$ columns of data contained in the THERML.DAT file (see Figure 8 ). The $\mathbf{x}$-axis can be scaled by the RSCL thermal parameter (Figure 14). A sample frequency histogram plot is shown in Figure 17.

The second option (F2) provides a graphical display of the intersection of rays with the dish surface. An example of the intersection of 2000 rays with the dish surface is shown in Figure 18. Symbols are used to indicate the radial zones in the target plane to which specific rays are reflected. The user can optionally select the maximum radial value in the target plane to be used for the zonal grouping of reflected rays. For example, from Figure 17, the radial distance at which some rays intersect the target plane may exceed $0.3 \mathrm{~m}$. However, most of the rays are contained within an aperture having a radius of $0.2 \mathrm{~m}$. Figure 18 was therefore generated for concentric radial zones out to $0.2 \mathrm{~m}$.

When the third option is chosen (F3), statistics associated with the intersection of rays in the target plane are displayed and the radius of the spot diagram in the target plane can be specified by the user (the default is to use the maximum radial excursion in the target plane). Once this has been done, a zonal spot diagram of the rays intersecting the target plane is displayed (Figure 19). As with the previous display, symbols are used to indicate the radial zone on the dish surface from which each ray was reflected. The maximum and RMS radius of the intersecting rays is indicated, as is the RMS deviation. 
Perîfect (analytical) parabolic dish: $f / 0=0.6$; Errors Included Frequency Histogram for 2098 Rays

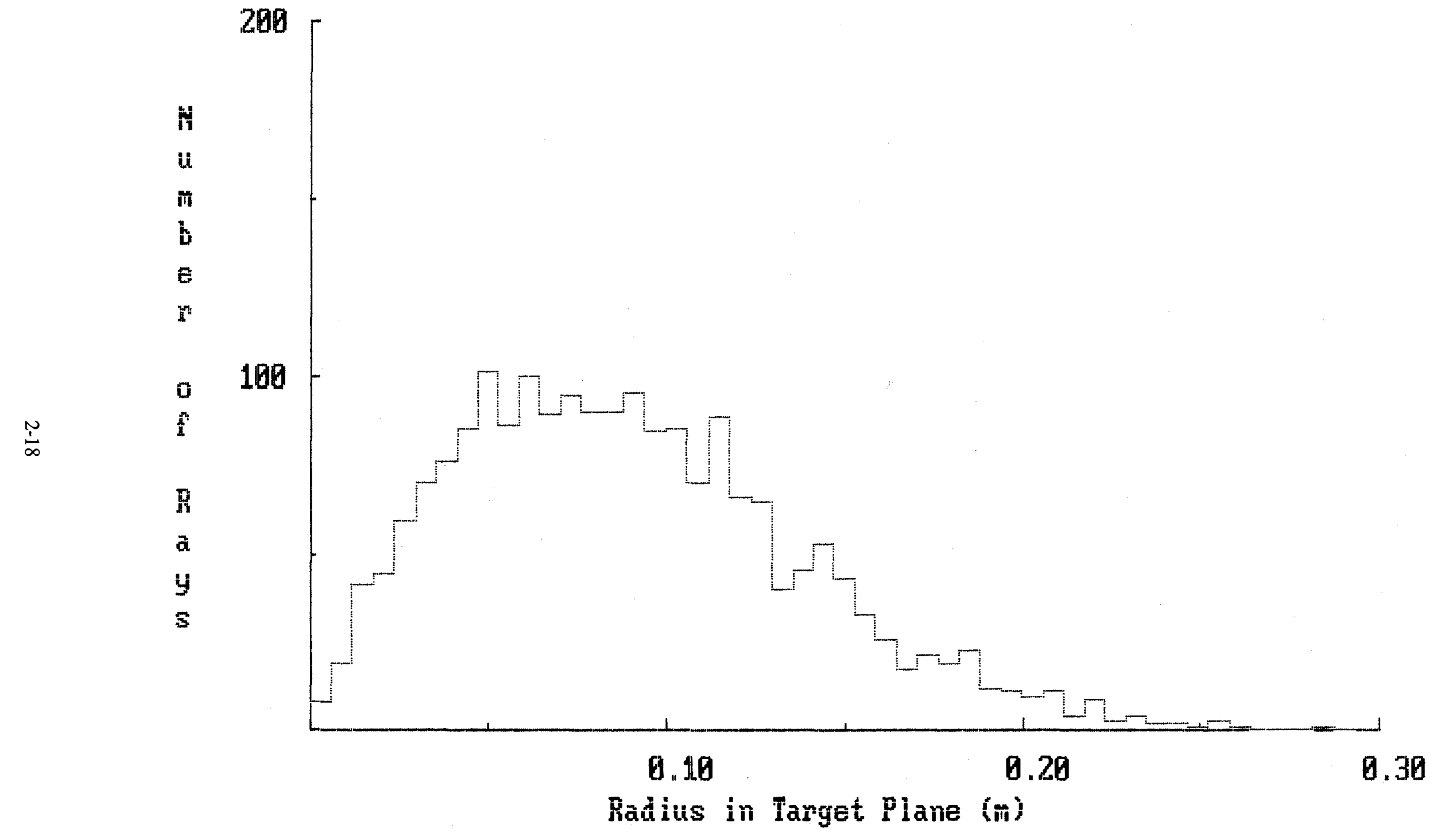

Figure 17. Sample frequency histogram 
Perfect (analytical) parabol ic dish; $f / D=0.6$; Errors Included Dish Radius= 7.50; Focal Length= 9.00; Dish Surface Location

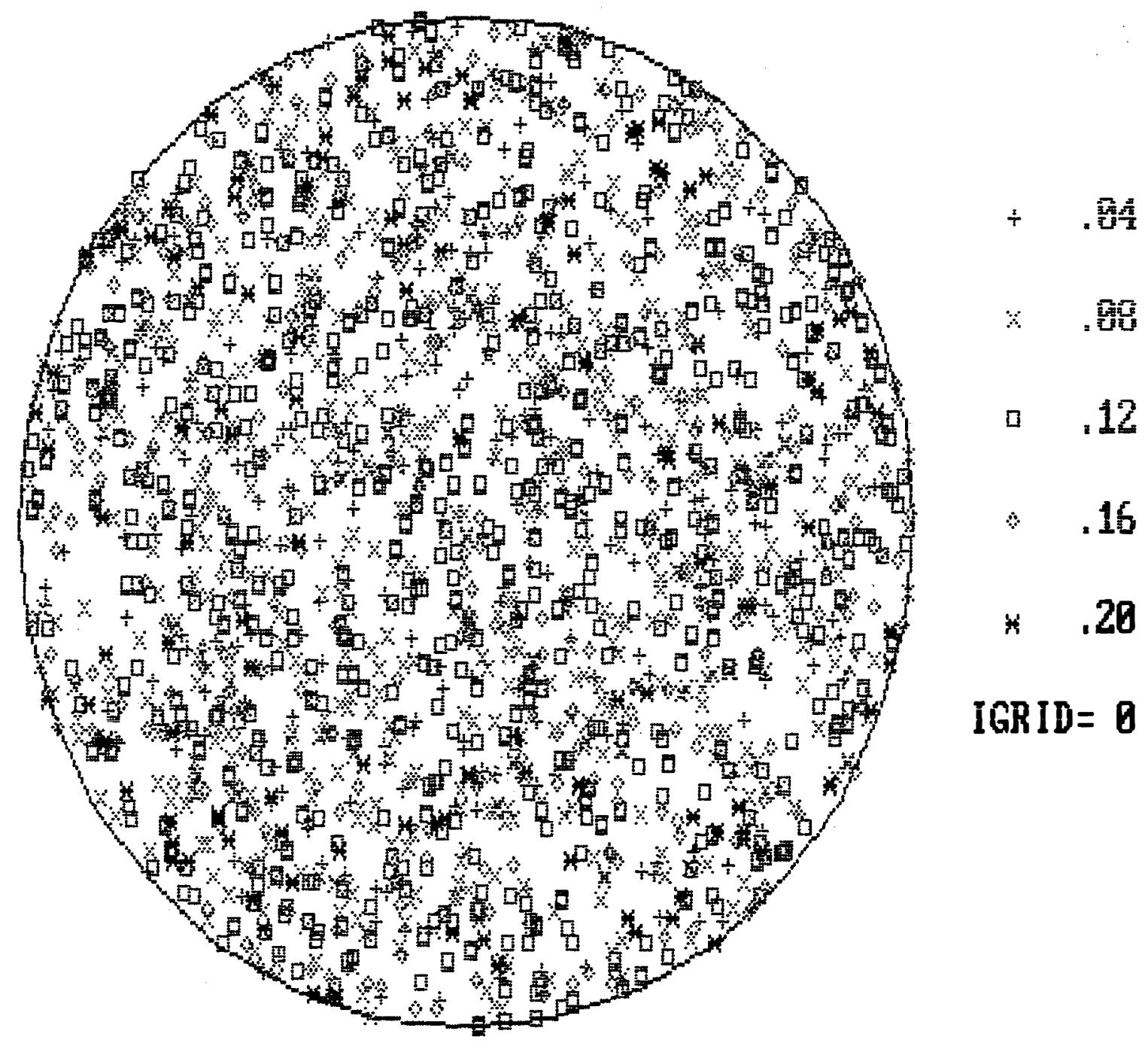

Figure 18. Distribution of randorly generated rays on the dish surface; zonal binning is out to $0.2 \mathrm{~m}$ in the target plane 
Perfect (analytical) parabolic dish; $f / D=8.6$; Errors Included Dish Radius $=7.50$; Focal Length $=9.80$; Target Distance $=9.80$

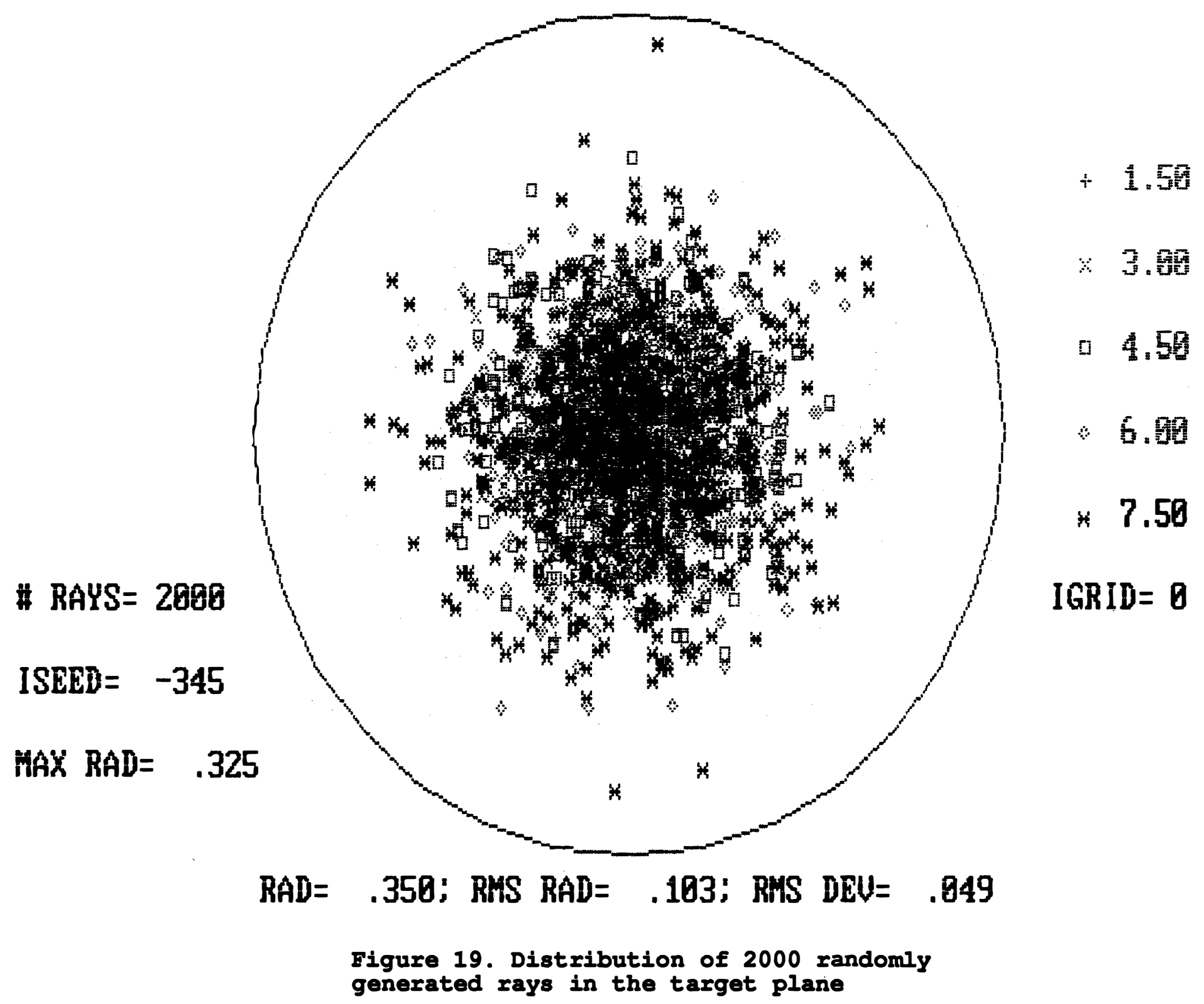


The final graphics option (F4) plots thermal efficiency as a function of aperture radius for the number of thermal analyses specified by the NTMP thermal parameter (Figure 14). Up to 4 temperatures can be run at a time; these temperatures (in ${ }^{\circ} \mathrm{C}$ ) are the first part of the legend shown at the lower right-hand corner (Figure 20). The second part of the legend indicates whether the efficiency curve is for a system with a primary only (1) or a system with both a primary and secondary (2). Efficiency plots can be generated which display primary only data, primary with secondary only data, or both primary and primary with secondary (F1 thru F3 in Figure 21 , respectively). The third piece of information contained in the legend is the radial value at which the efficiency is a maximum.

If a printer is active (IPRT=2 in Figure 12), optional hardcopy of the various graphical displays can be generated by responding "Y" to the "Hardcopy Desired?" query. Note that if an EGA graphics adapter is in use (IGRF=3 in Figure 12), a screen dump utility (such as GRAFPLUS from Jewell Technologies, Inc.) must have been installed prior to running OPTDSH.

Once the graphics menu is exited, the data file menu is modified to allow archival of the traced ray information (Figure 22). By choosing F5, a file name is requested and a . RAY file is created.

Interface with the DISH Structural Code

The interface with the DISH structural code is through files having .SHP extensions. Typically, these are DISH.DAT files created by the DISH program which have been renamed to have a .SHP extension. It should be emphasized that this interface is analytical in the sense that the position and slopes of the dish surface at each ray intersection point is computed exactly; no interpolation is required. An example. SHP file is shown in Figure 23. The meaning of the various input values are described in the main body of this report. 
Perfect (analytical) parabolic dish; $f / D=0.6$; Errors Included

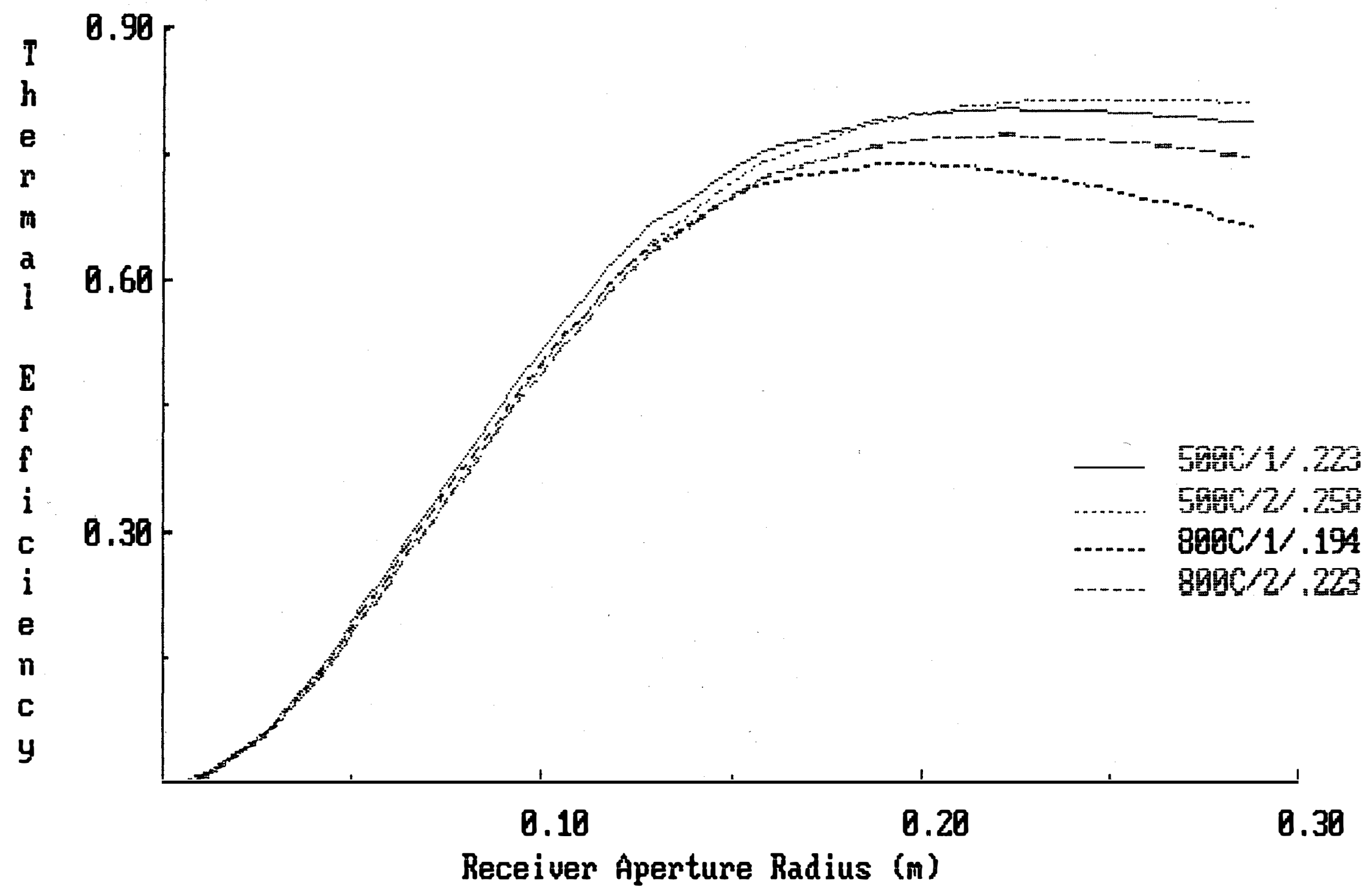

Figure 20. Thermal efficiency as function of aperture radius 
Select Thermal Efficiency Graphics Option:

F1: Primary

F2: Primary with Secondary

F3: Primary + Primary with Secondary

F4: Return to Previous Menu

Enter F-Key Choice:

Figure 21. Thermal efficiency plot options

Select Data File Option:

F1: Finite Element Data (.DAT)

F2: Shape Data (.SHP)

F3: Ray Data (.RAY)

F4: Re-Process Previouly Traced Rays

F5: Archive Traced Rays

F6: Return to Previous Menu

Enter F-Key Choice:

Enter name of desired output .RAY file (maximum of 6 characters), prefaced by an optional disk drive designator (default is C:): PARAB

Ray File (C:PARAB.RAY) Being Archived ....

Figure 22. Archival of .RAY file 
Axisymmetric edge pull, $10 \mathrm{mil}$ composite, simply-supported.

1
$2.540000 \mathrm{E}-04$
$3.000000 \mathrm{E}-01$
$0.000000 \mathrm{E}+00$
3.141593
$3.846154 \mathrm{E}-01$
423.487000
0
$0.000000 \mathrm{E}+00$
$0.000000 \mathrm{E}+00$
$3.330905 \mathrm{E}-05$
$0.000000 \mathrm{E}+00$
$1.227963 \mathrm{E}-03$
$-6.233433 \mathrm{E}-02$
$0.000000 \mathrm{E}+00$
$0.000000 \mathrm{E}+00$
$0.000000 \mathrm{E}+00$
1.000000
$0.000000 \mathrm{E}+00$
$0.000000 \mathrm{E}+00$
$0.000000 \mathrm{E}+00$
$0.000000 \mathrm{E}+00$
19397.810000
42764.710000
$4.569858 \mathrm{E}-05$

1

$10 \quad 5 \quad 0$

$2.540000 \mathrm{E}-04 \quad 7.500000$

3.120000

$2.123141 \mathrm{E}-02$

$7.686390 \mathrm{E}-05$

18.000000

2

1

$0.000000 \mathrm{E}+00$

0. $000000 \mathrm{E}+00$

0. $000000 \mathrm{E}+00$

1.000000

$0.000000 \mathrm{E}+00$

0. $000000 \mathrm{E}+00$

$-4.149304 \mathrm{E}-01$

0. $000000 \mathrm{E}+00$

0. $000000 \mathrm{E}+00$

0. $0000000 \mathrm{E}+000$

0. $000000 \mathrm{E}+00$

0. $000000 \mathrm{E}+00$

751.720200

9.000000

$7.930000 \mathrm{E}+09$

689.700000

0. $000000 \mathrm{E}+00$

$0.000000 \mathrm{E}+00$

$0.000000 \mathrm{E}+00$

3. $947911 \mathrm{E}-01$

19.500000

$9.230769 \mathrm{E}-01$

1

2014220.000000
() $0.000000 \mathrm{E}+000$
0. $000000 \mathrm{E}+00$

(). $000000 \mathrm{E}+00$

0. $000000 \mathrm{E}+00$

1

1

$-9.075131 \mathrm{E}-08$

0. $0000000 \mathrm{E}+00$

0. $0000000 \mathrm{E}+000$

$3.765548 \mathrm{E}-08$

$0.000000 \mathrm{E}+000$

1.000000

0. $000000 \mathrm{E}+00$

0. $0000000 \mathrm{E}+00$

$-5.116470 \mathrm{E}-044$

$0.000000 \mathrm{E}+00$

9.574924E-01

0. $000000 \mathrm{E}+00$

0. $0000000 \mathrm{E}+00$

0. $000000 \mathrm{E}+00$

0. $000000 \mathrm{E}+00$

0. $000000 \mathrm{E}+00$

0. $0000000 \mathrm{E}+00$

o. $0(10000 \mathrm{E}+00$

o. $000000 \mathrm{E}+00$

(). $000000 \mathrm{E}+00$

0. $000000 \mathrm{E}+00$

0. $000000 \mathrm{E}+00$

0. $000000 \mathrm{E}+00$

$8.875375 \mathrm{E}-01$

0. $000000 \mathrm{E}+00$

0. $000000 \mathrm{E}+00$

0. $000000 \mathrm{E}+00$

46554.750000

0. $000000 \mathrm{E}+00$

$-9196.523000$

0. $000000 \mathrm{E}+00$

$-4.042378 \mathrm{E}-0.2$

$0.000000 \mathrm{E}+00$

0. $000000 \mathrm{E}+00$

4. $042372 \mathrm{E}-02$

0. $000000 \mathrm{E}+00$

Figure 23. Example of DISB.DAT input file used with SBAPE option 


\section{REFERENCES}

Grafmatic Scientific/Engineering Graphics (1984). Microcompatibles, Inc., CGA/EGA version for use with Microsoft 4.0 FORTRAN.

Kutscher, C. (1987). "Optical Figure of Merit for an Axisymmetric Reflector," Internal Memo, Solar Energy Research Institute, Golden, CO.

Lewandowski, A., (1987). "Dish Receiver Heat Loss Update", Internal Memo, Solar Energy Research Institute, Golden, CO.

Malacara, D. (1978). Optical Shop Testing, John Wiley and Sons, New York, p. 494.

Press, W.H., et. al. (1987). Numerical Recipes, The Art of Scientific Computing, Cambridge University Press, Cambridge, pp. 95-101.

O'Gallagher, J. and Winston, R. (1987). "Performance and Cost Benefits Associated with Nonimaging Secondary Concentrators Used in Point-Focus Dish Solar Thermal Applications," SERI Subcontractor Report No. SERI/STR-253-3113 for Subcontract No. XK-4-04070-03.

Spencer, G.H. and Murty, M.V.R.K. (1962). "General Ray-Tracing Procedure," J. Opt. Soc. Am., Vol. 52, No. 6, pp. 672-678.

Wood, R. (1988). "Scanning Hartmann Optical Tester Preliminary Design Report," Engineering Note, Solar Energy Research Institute, Golden, Co. 


\section{Appendix A}

\section{Asymptotic Solutions for Edge Effects in Thin Shells}

presented February 10, 1988

to the SERI Membrane Dish Workshop

by C. R. Steele and C. D. Balch

Division of Applied Mechanics

Stanford University

Stanford, California 94305 


\section{Current Work with SERI}

Goal:

To develop asymptotic shell solutions and incorporate them into design formulas and software for the stress and displacement analysis of thin dish reflectors.

\section{Scope:}

- Initially curved, thin dish, i.e., a paraboloid.

- Elastic behavior, small strains.

- High prestress due to pressure loading.

- Edge effects due to support conditions.

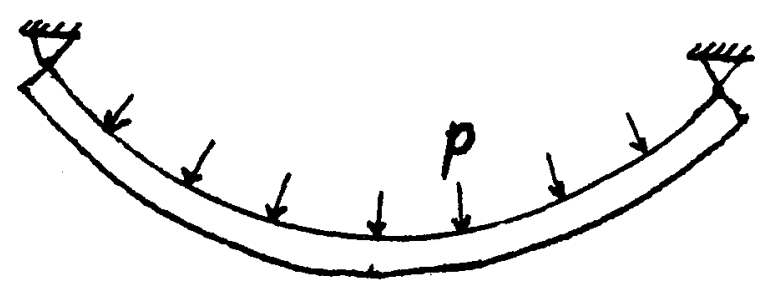




\section{General Approach to Shell Problems}

Total Solution

(for given load \& B.C.'s)

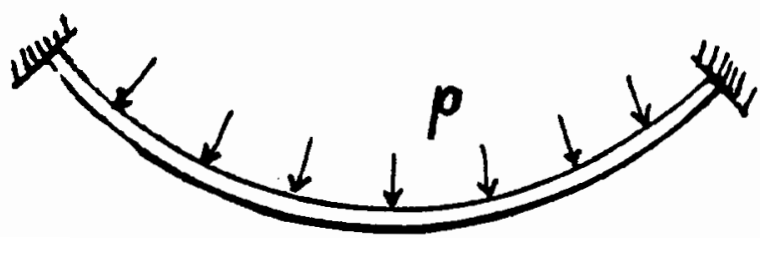

II

Particular Solution (Membrane solution: no bending moment or transverse shear.)
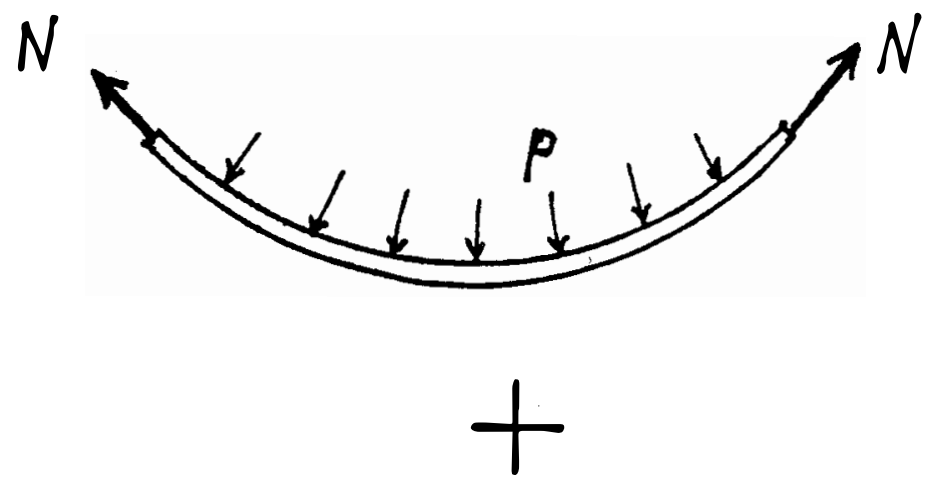

Complementary Solution (Edge effect: has local bending and shear.)

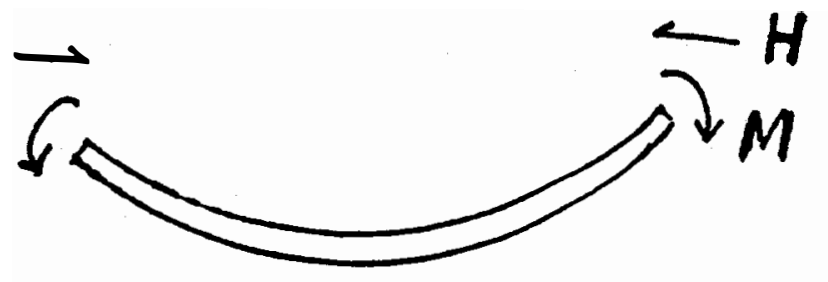

The edge effect is needed to satisfy the B.C.'s. 
$\underline{\text { Axisymmetric Shell Problems }}$

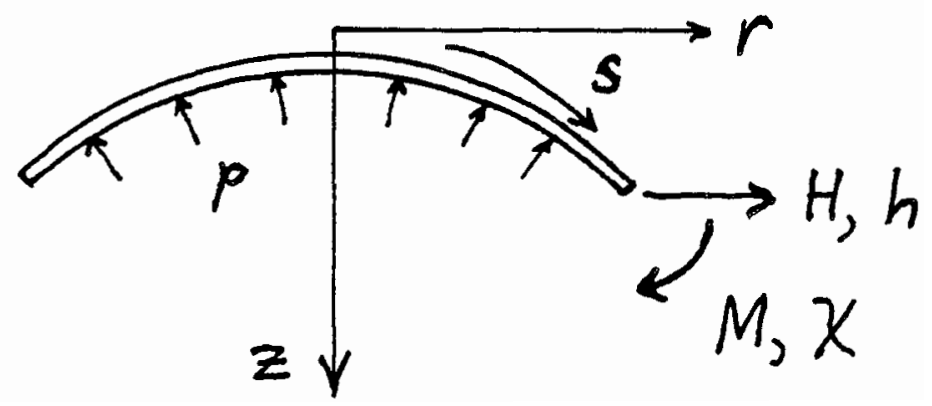

Fourth-order system, similar to a beam:

$-\frac{d}{d s}\left[\begin{array}{c}M \\ H \\ \chi \\ h\end{array}\right]+[A(s)]\left[\begin{array}{c}M \\ H \\ \chi \\ h\end{array}\right]=[\cdot \mathbf{P}]$

$=\left[\begin{array}{l}0 \\ 0 \\ 0 \\ 0\end{array}\right]$ for edge loads alone

The coefficients $A(s)$ are functions of position. 
The Prestress, or Pressurization Effect

Beam:

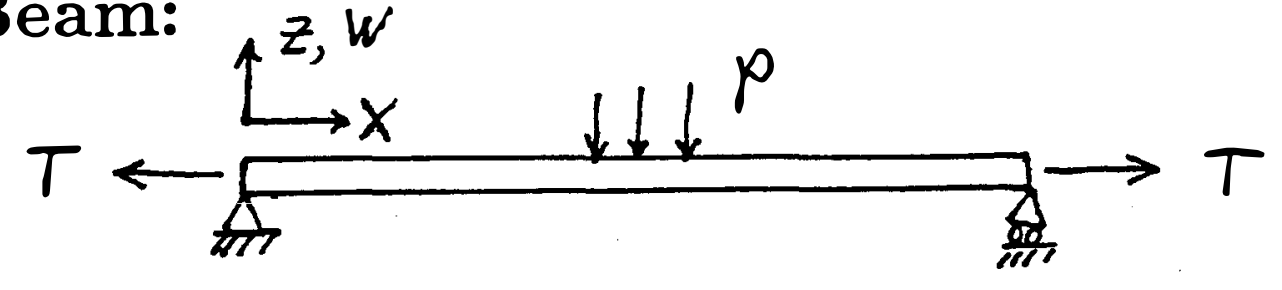

$$
E I \frac{d^{4} w}{d x^{4}}=p+T \frac{d^{2} w}{d x^{2}}
$$

Shell of Revolution: Modify one component of the matrix $[A]$ in the shell equations.

The new nonlinear term contains the dimensionless stress quantity

$$
\rho=\frac{N R}{2 E t c}
$$

$\rho \gg 1 \quad \Longrightarrow \quad$ high tensile prestress

$-1<\rho<1 \Longrightarrow$ low to moderate prestress

$$
\rho=-1 \quad \Longrightarrow \quad \text { local buckling of the shell }
$$




\section{Asymptotic Solutions for Shell Edge Effects}

WKB Method, with large parameter

$$
\lambda=\sqrt{\frac{R}{t}} \gg 1
$$

Dimensionless solution form:

$\left[\begin{array}{l}\overline{\bar{M}} \\ \overline{\bar{\chi}} \\ \bar{h}\end{array}\right]=\underbrace{e_{\text {rapid variation }}^{-\lambda \xi(s)}}_{\{}\left[\Phi_{0}+\frac{1}{\lambda} \Phi_{1}+\frac{1}{\lambda^{2}} \Phi_{2}+\ldots\right]$

Steps of the method:

(i) Substitute above form into shell equations.

(ii) Collect terms in each power of $\lambda$.

(iii) Obtain an eigenvalue problem for $\xi^{\prime}(s)$.

(iv) Eigenvalues are the decay constants for the edge effects. 


\section{Asymptotic Solutions: The WKB Method}

WKB Method: Suited for equations with variable coefficients and rapidly varying solutions.

Example: High frequency vibration of a string with variable density $m(x)$.

Equation : $\quad T \frac{d^{2} w}{d x^{2}}+\omega^{2} m(x) w=0$

SLOWLY

VARYING ENVELOPE

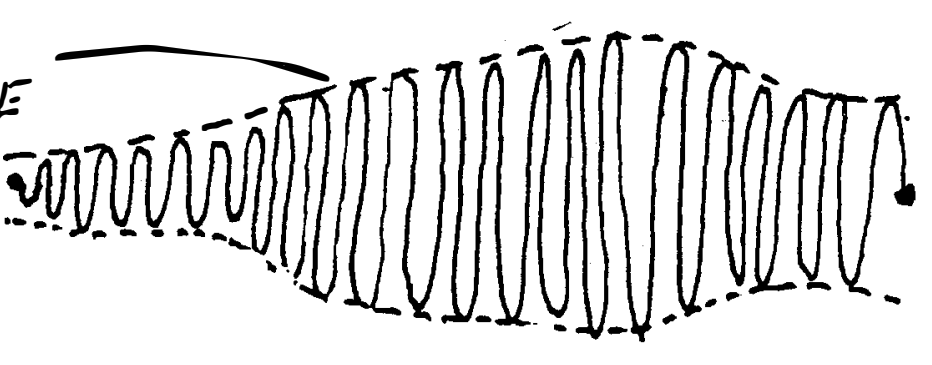

We have the large parameter

$$
\lambda=\frac{\omega^{2} M L}{T} \gg 1
$$

For very high frequencies -

FDM, FEM: Mesh $\rightarrow 0$; Cost $\rightarrow \infty$. WKB: Cost $\rightarrow 0$. 


\section{$\underline{\text { Decay Behavior of the Edge Effects }}$}

For low membrane prestress; $\rho=\frac{N R}{2 E t c}=O(1)$ :

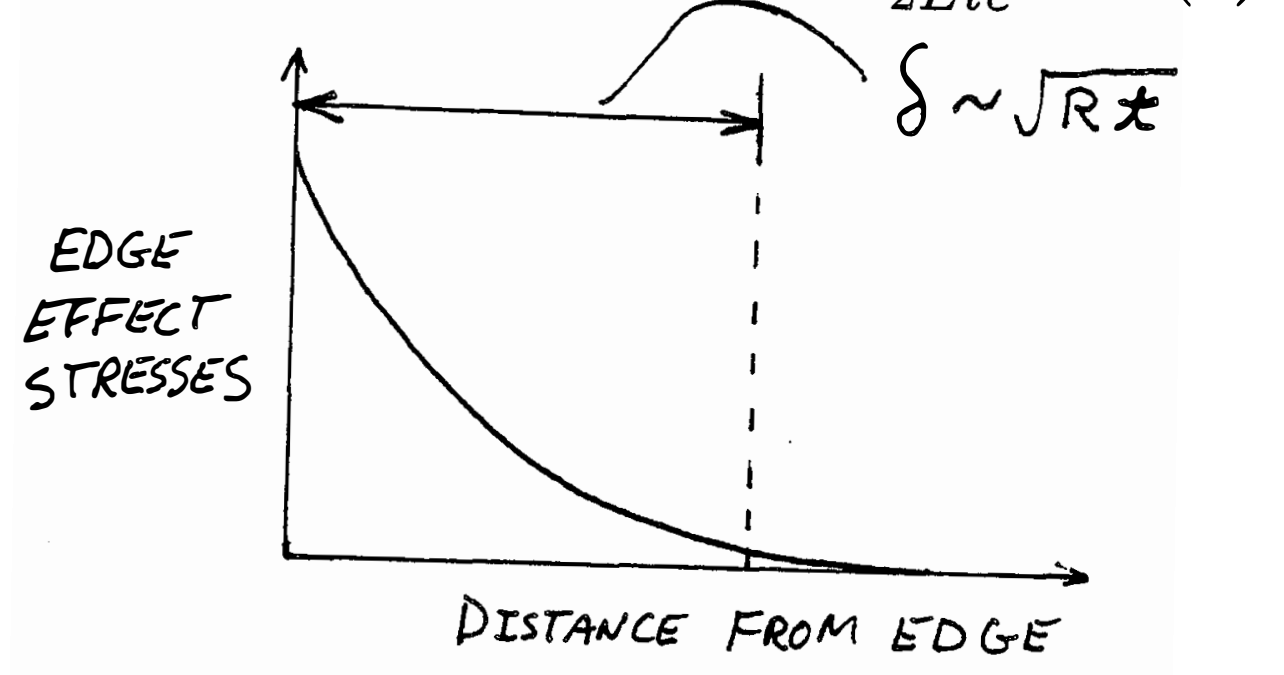

For high membrane prestress; $\rho=\frac{N R}{2 E t c} \gg 1$ :

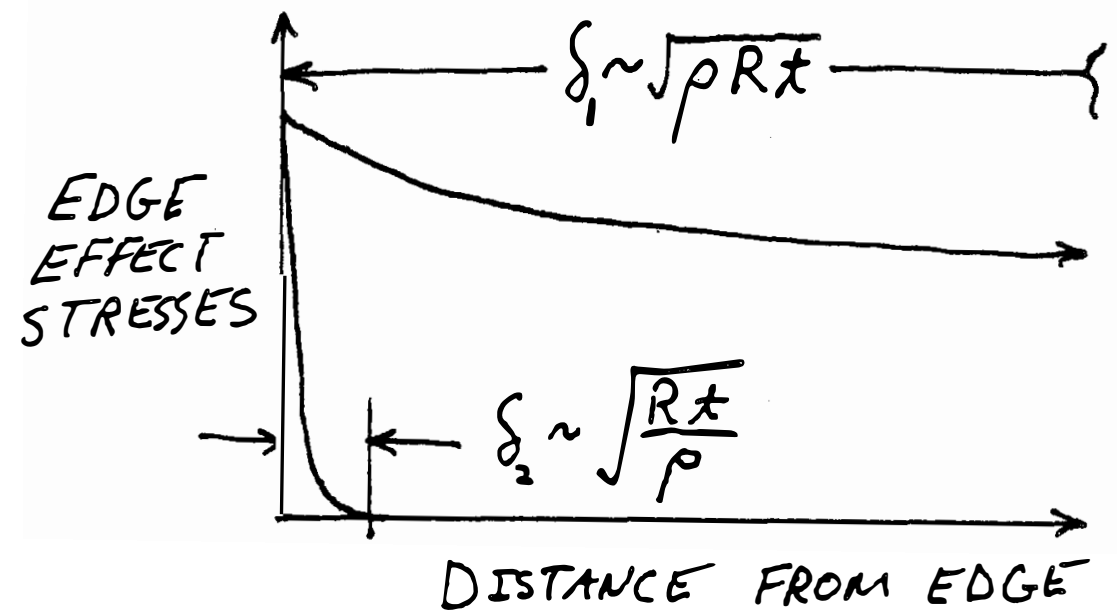

(two edge-effect solutions) 


\section{Example: Pressurized Paraboloid}

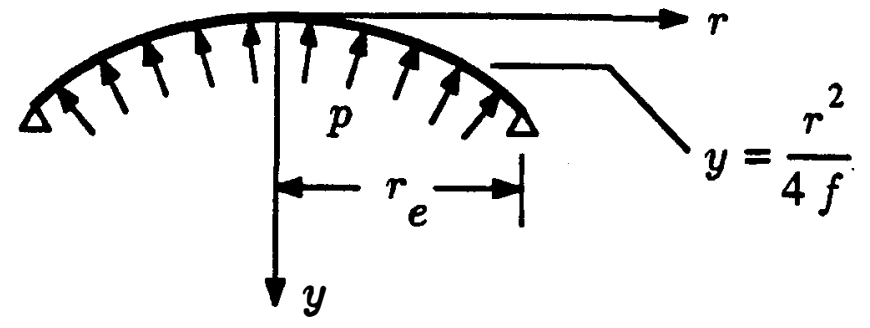

Paraboloidal shell with focal length $f$; subjected to a uniform pressure load $p$. The meridian curve is the parabola $y=r^{2} /(4 f)$.

Specifications for paraboloid, from Murphy (1987).

\begin{tabular}{|l|c|c|}
\hline \multicolumn{1}{|c|}{ Geometry } & Material Properties & Load \\
\hline$t=0.254 \mathrm{~mm}$ & $E=209 \mathrm{GPa}$ & $p=2000 \mathrm{~Pa}$ \\
$r_{\mathrm{e}}=7.5 \mathrm{~m}$ & $\nu=0.3$ & \\
$f=9 \mathrm{~m}$ & & \\
\hline
\end{tabular}




\section{Rotation of the Shell Surface}

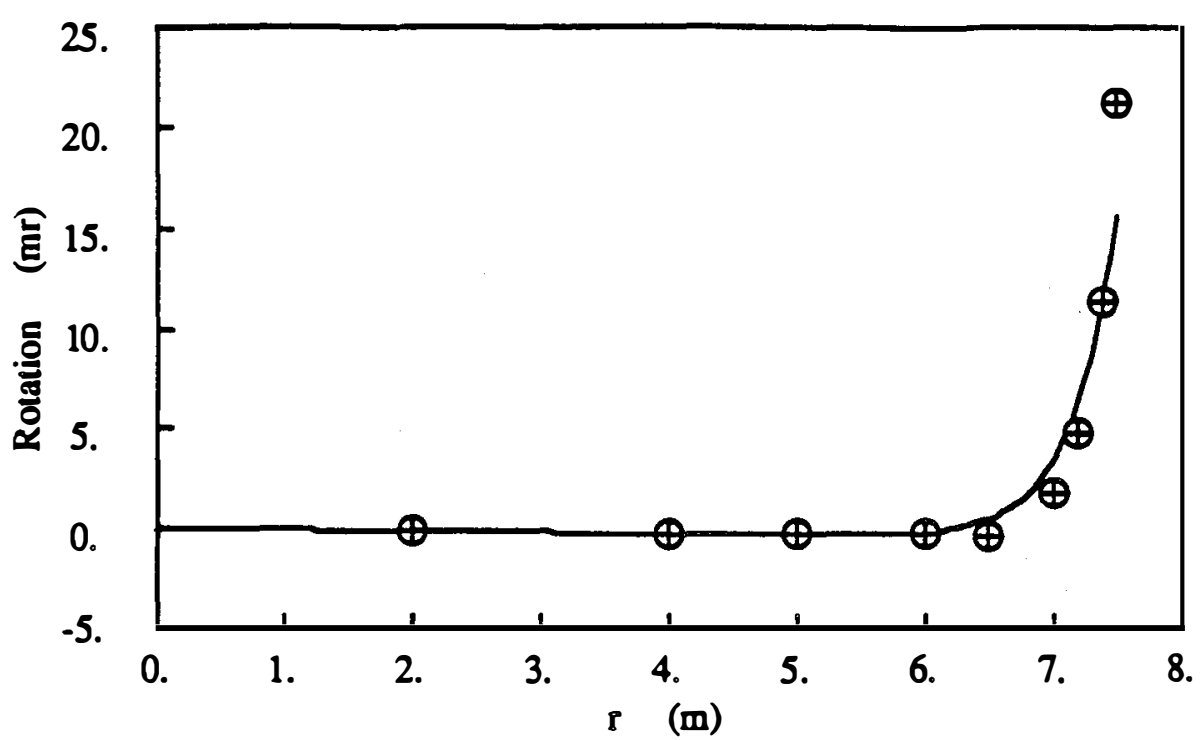

Rotation $\chi$ as a function of radial distance $r$ for the simply supported parabolic shell under pressure load. The solid line is the asymptotic solution; cross marks are ANSYS results from Murphy (1987).

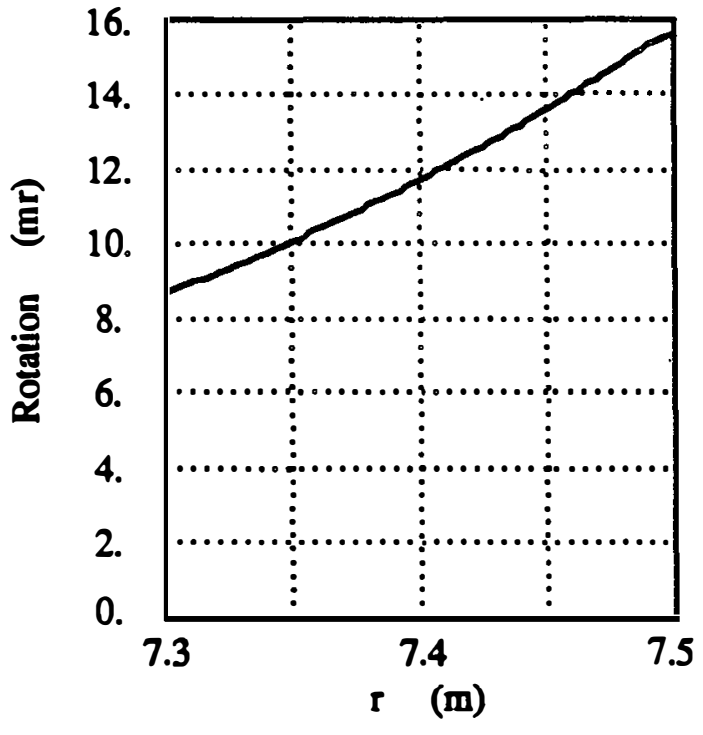

(a)

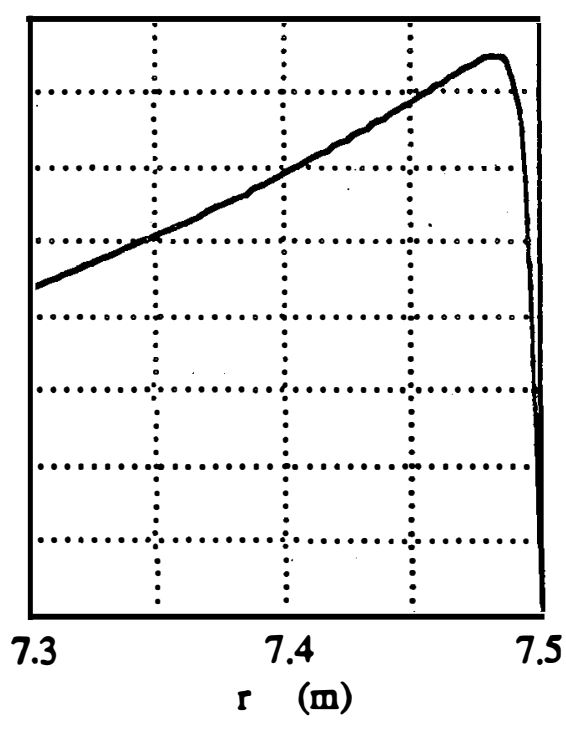

(b)

Asymptotic solution for the meridional rotation $\chi$ near the edge of the parabolic shell under pressure load. (a) Simply supported edge: the meridional rotation reaches a maximum value at the edge $r=7.5 \mathrm{~m}$. (b) Built-in edge: the meridional rotation is zero at the edge. 


\section{Bending Stress (Simply Supported Edge)}

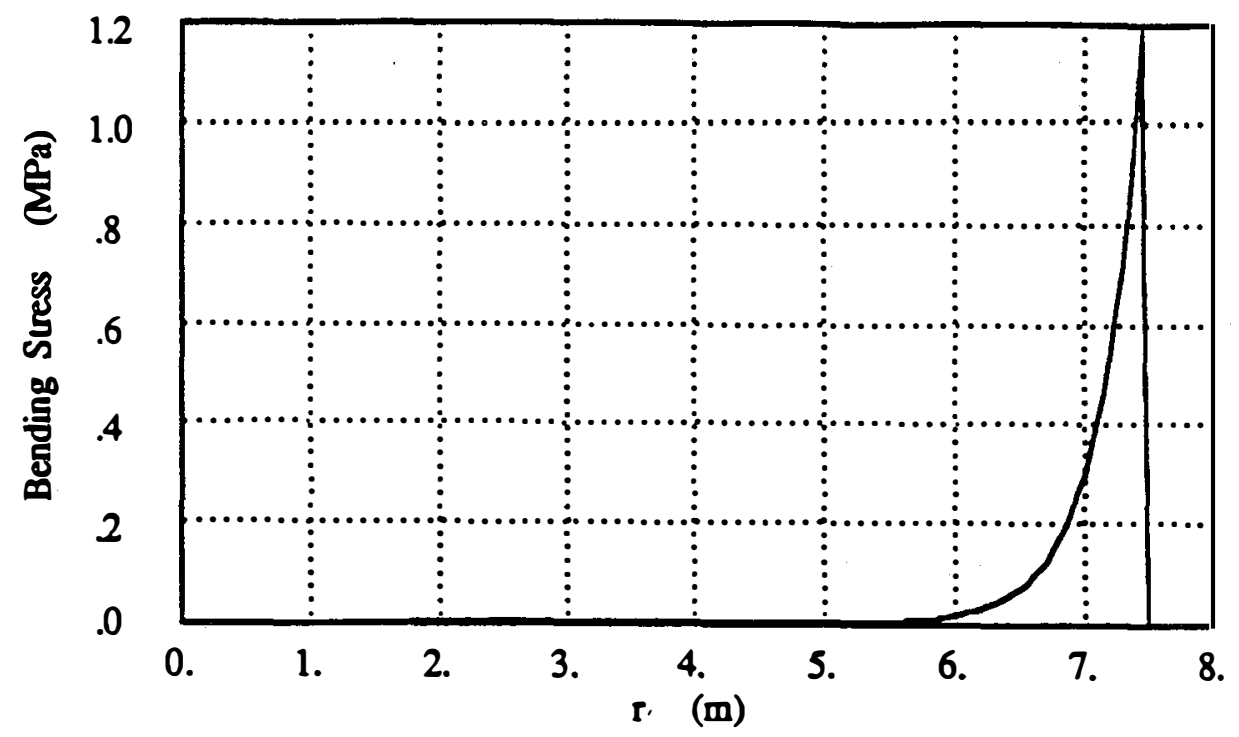

Asymptotic solution for the meridional bending stress in a simply supported parabolic shell under pressure load. The bending stress $\sigma_{\phi \mathrm{B}}$ is shown as a function of the radial distance $r$.

\section{Displacements (Simply Supported Edge)}

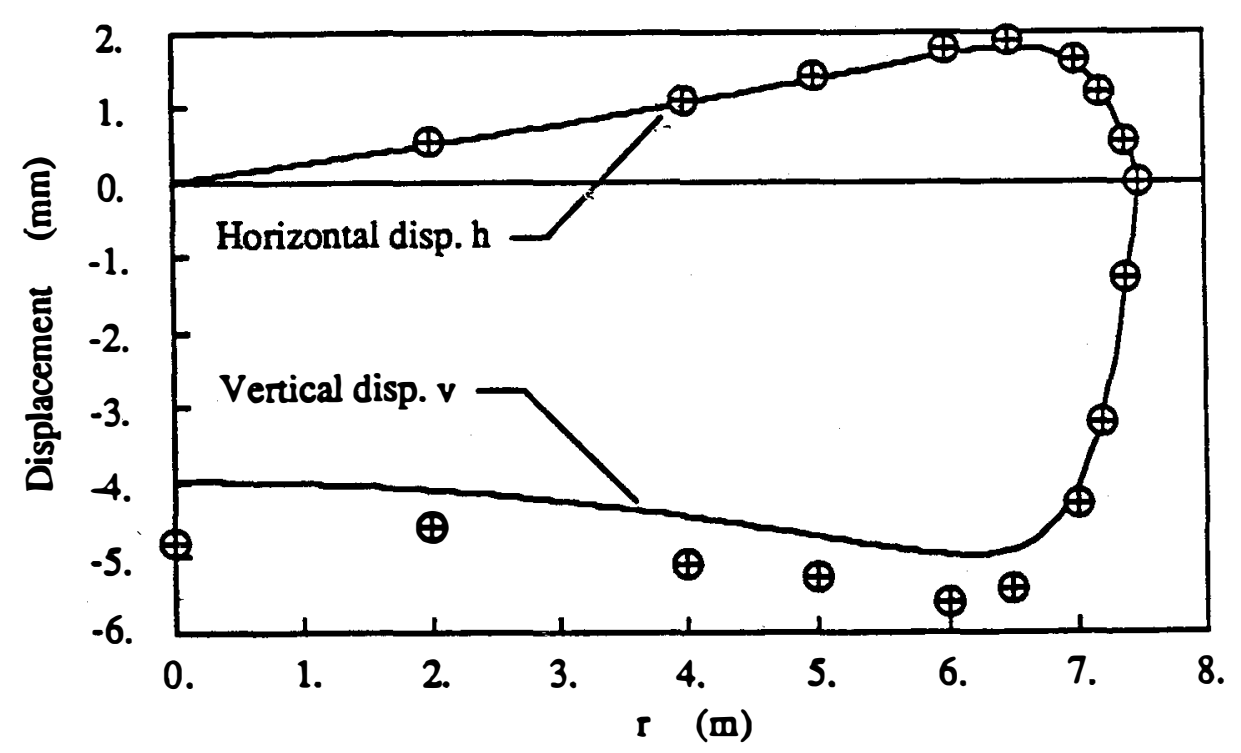

Horizontal displacement $h$ and vertical displacement $v$ for the simply supported parabolic shell under pressure load. Solid lines are the asymptotic solution; cross marks are ANSYS results from Murphy (1987). 
Future Work: Non-axisymmetric Behavior

Discrete Supports

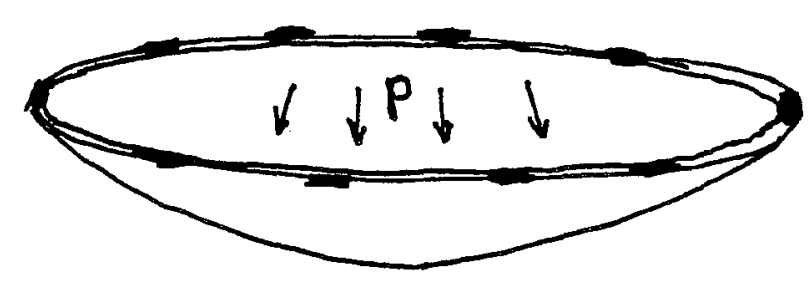

Wavy Edge Displacement

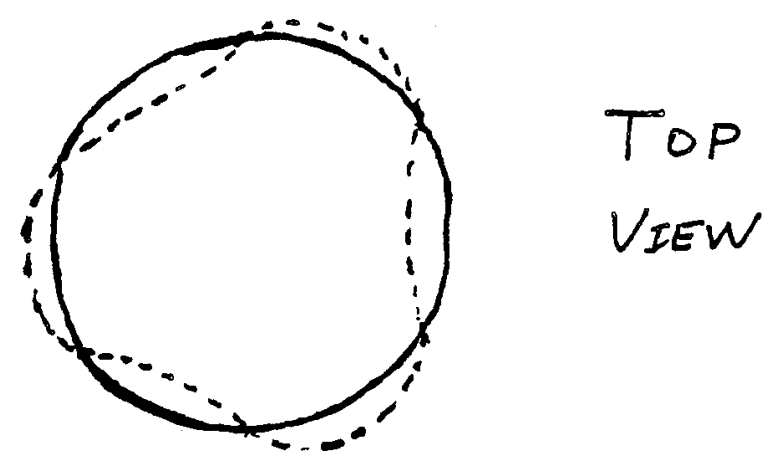

Non-axisymmetric Loads, e.g., Wind

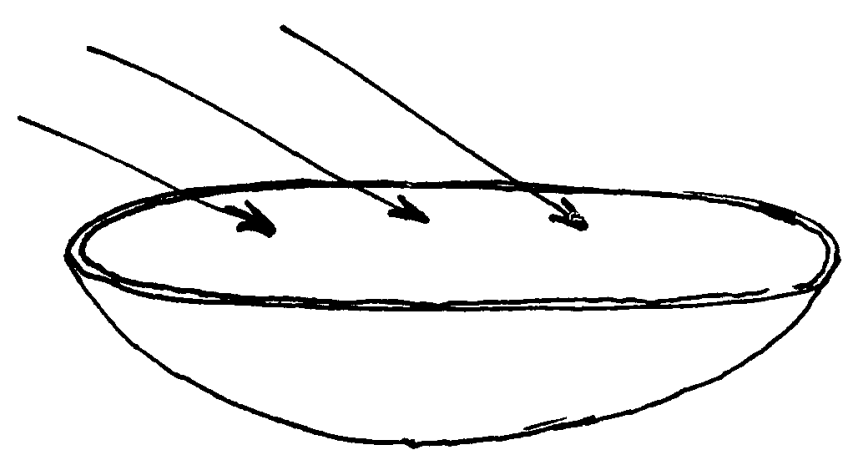




\section{Preliminary Results for Non-axisymmetric Edge Effects}

Shallow parabolic shell:

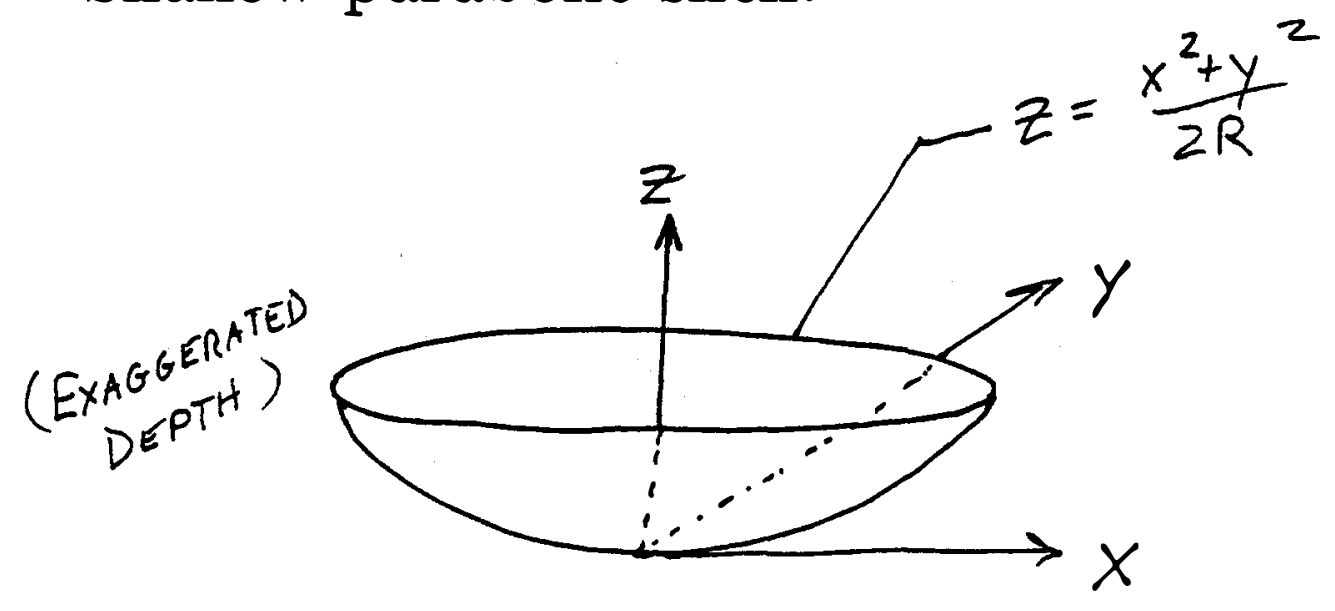

Assume large prestress $\rho \gg 1$.

The shallow shell equations for edge effects reduce to

$$
D \Delta \Delta w-N \Delta w+\frac{E t}{R^{2}} w=0
$$




\section{Asymptotic Solution \\ for High Circumferential Harmonics}

$$
\begin{gathered}
w=\cos n \theta e^{n \xi(r)}\left(w_{0}+\frac{1}{n} w_{1}+\frac{1}{n^{2}} w_{2}+\ldots\right) \\
(n \gg 1) \\
\\
\qquad y
\end{gathered}
$$

Decay distances of edge effects are:

$$
\begin{gathered}
\delta_{\text {Long }} \sim \sqrt{\frac{R t}{1+\frac{R}{t} \frac{1}{2 n^{2} \rho}}}<\sqrt{R t} \\
\delta_{\text {Short }} \sim \sqrt{\frac{R t}{1+\frac{R}{t} \frac{2 \rho}{n^{2}}}}<\sqrt{R t}
\end{gathered}
$$

High circumferential harmonics die out fast, even with large prestress.

Next Step: Apply this approach to the equations in matrix form, and solve non-axisymmetric shell problems by Fourier series. 


\section{Concluding Remarks}

- Asymptotic solutions have been derived for edge effects in thin parabolic shells loaded by pressure. These solutions compare favorably with ANSYS finite element results, and capture extremely rapid stress variations near the edge of the shell.

- The asymptotic solutions provide the "decay distances" for edge effects, and thus offer physical insight into the shell's behavior.

- Prestress, or high tension of the shell, is found to significantly effect the decay distances. Preliminary calculations suggest that the most significant prestress effects are axisymmetric.

- The membrane and edge-effect solutions for shells of revolution can be employed for rapid computerized analysis and design studies. 


\section{Appendix B}

\section{Non-Axisymmetric Deformations of Thin Paraboloidal Shells with Initial Prestress}

presented May 12, 1988

to the SERI Membrane Dish Research Group

by C. D. Balch and C. R. Steele

Division of Applied Mechanics

Stanford University

Stanford, California 94305 


\section{Current Work with SERI}

\section{Goal:}

To develop analytical solutions for the nonaxisymmetric structural response of a thin, paraboloidal shell, and to incorporate the solutions into design formulas and software.

\section{Scope:}

- Initially paraboloidal dish.

- Elastic behavior, small strains.

- High prestress due to pressure loading.

- Non-axisymmetric edge effects.

- Non-axisymmetric loads, e.g., wind load. 


\section{General Approach to Shell Problems}

Total Solution

(for given load \& B.C.'s)

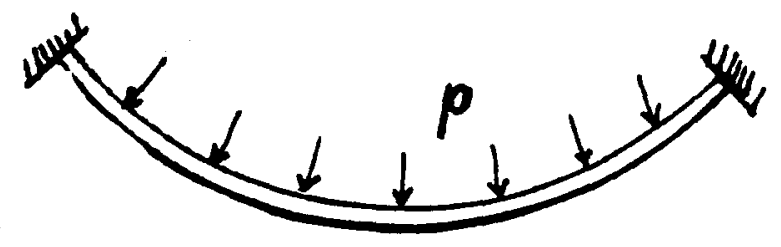

11

Particular Solution (Membrane solution: no bending moment or transverse shear.)
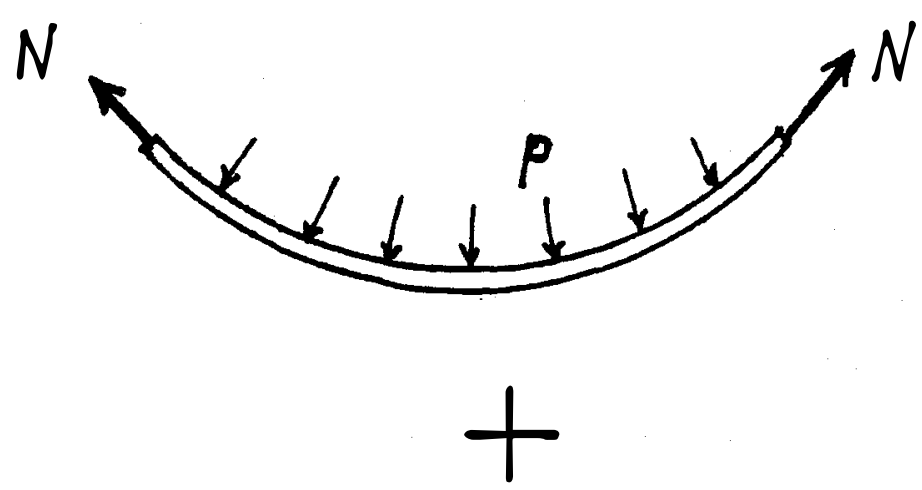

Complementary Solution (Edge effect: has local bending and shear.)

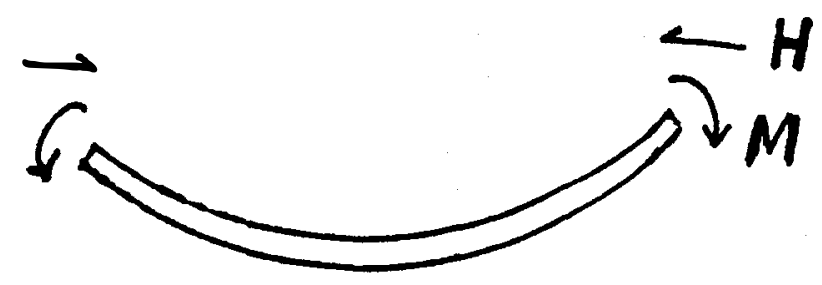

The edge effect is needed to satisfy the B.C.'s. 


\section{$\underline{\text { Axisymmetric Shell Problems }}$}

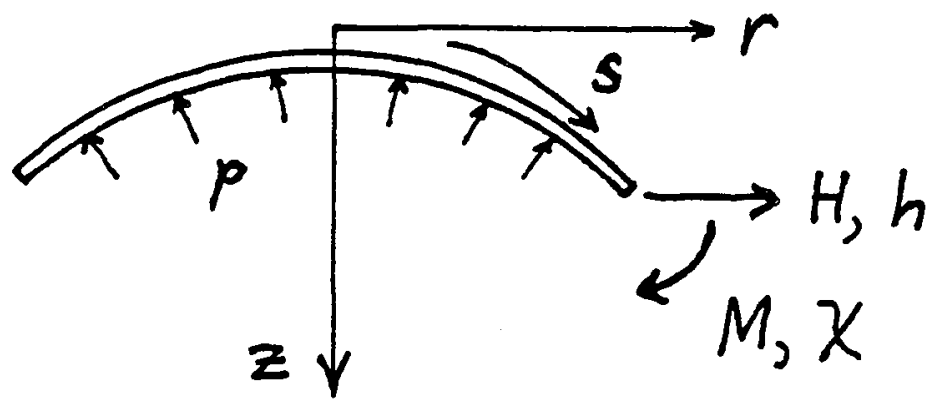

Fourth-order system, similar to a beam:

$-\frac{d}{d s}\left[\begin{array}{c}M \\ H \\ \chi \\ h\end{array}\right]+[A(s)]\left[\begin{array}{c}M \\ H \\ \chi \\ h\end{array}\right]=[\mathbf{P}]$

$=\left[\begin{array}{l}0 \\ 0 \\ 0 \\ 0\end{array}\right]$ for edge loads alone

The coefficients $A(s)$ are functions of position. 


\section{The Prestress, or Pressurization Effect}

Beam:

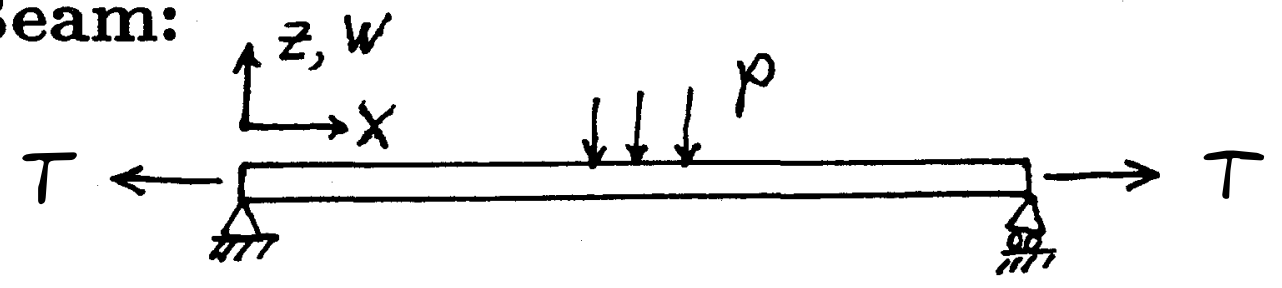

$$
E I \frac{d^{4} w}{d x^{4}}=p+T \frac{d^{2} w}{d x^{2}}
$$

Shell of Revolution: Modify one component of the matrix $[A]$ in the shell equations.

The new nonlinear term contains the dimensionless stress quantity

$$
\rho=\frac{N R}{2 E t c}
$$

$\rho \gg 1 \quad \Longrightarrow \quad$ high tensile prestress

$-1<\rho<1 \quad \Longrightarrow \quad$ low to moderate prestress

$$
\rho=-1 \quad \Longrightarrow \quad \text { local buckling of the shell }
$$




\section{Non-axisymmetric Problems: Equilibrium}
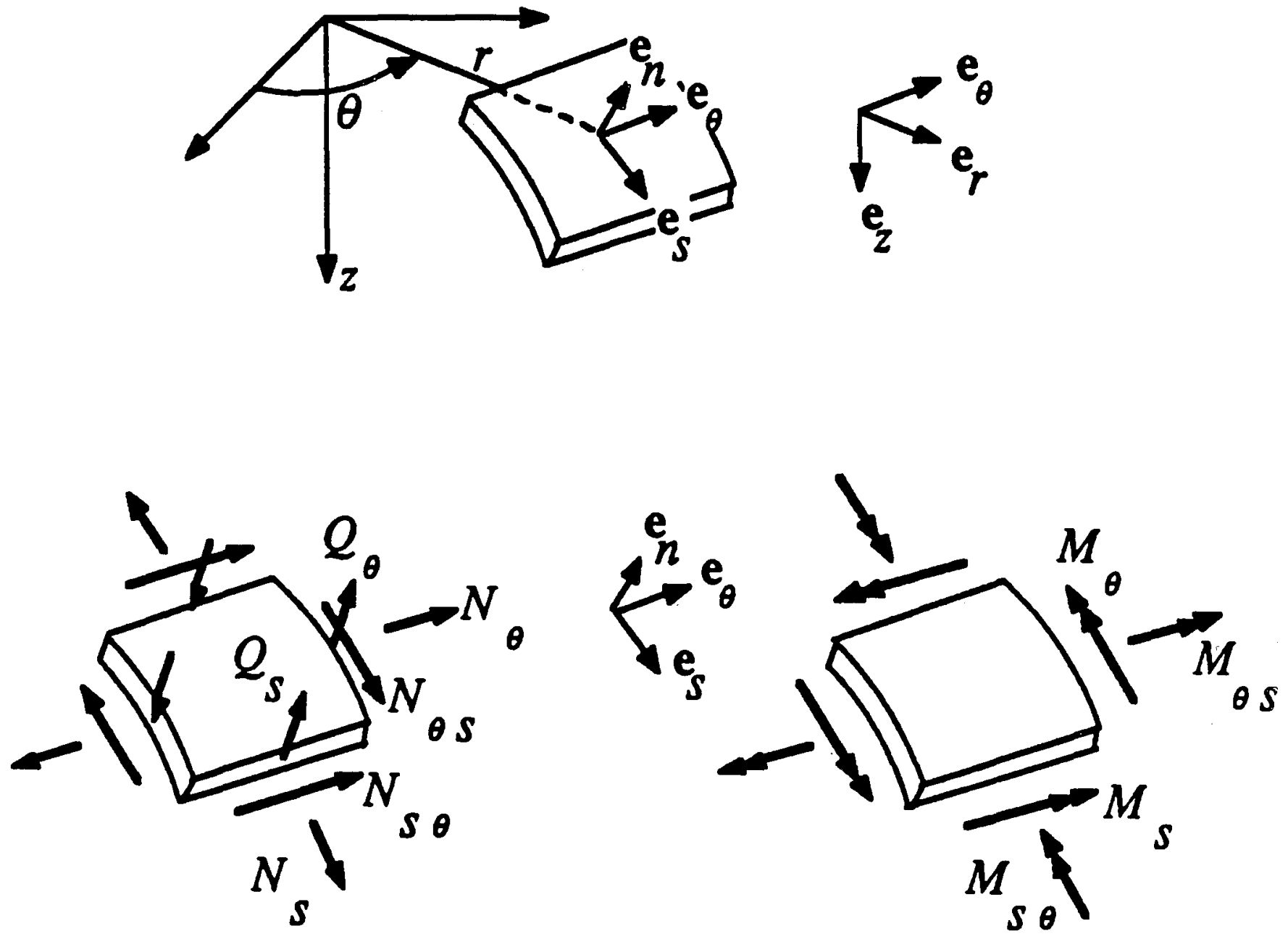
$\underline{\text { Non-axisymmetric Problems: Kinematics }}$
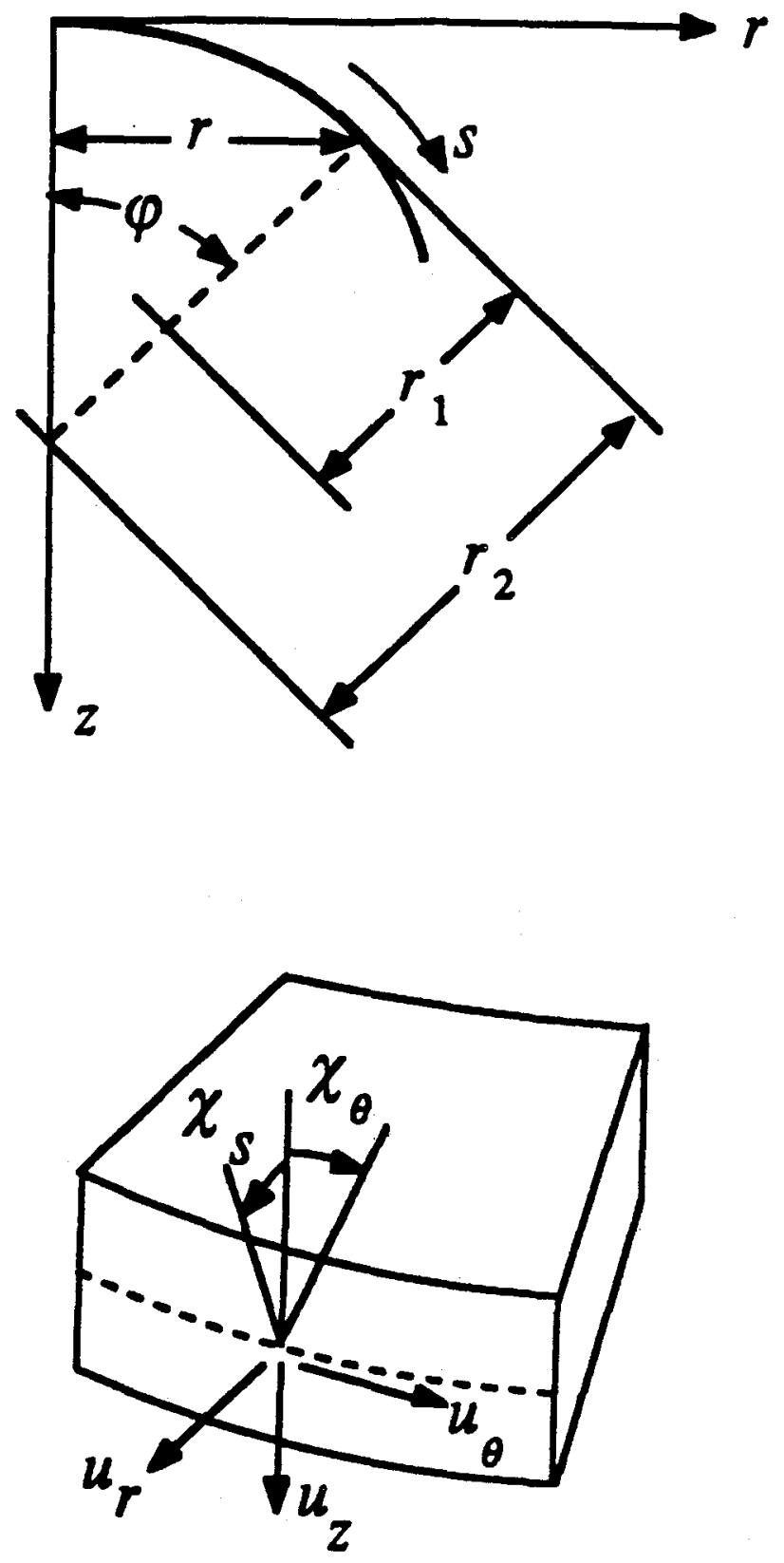
$\underline{\text { Non-axisymmetric Problems: Governing Equations }}$

Displacement and Force Quantities:

$$
\mathbf{D}=\left[\begin{array}{c}
\chi_{s} \\
\chi_{\theta} \\
u_{r} \\
u_{z} \\
u_{\theta}
\end{array}\right]^{(n)} \quad ; \quad \mathbf{F}=\left[\begin{array}{c}
r M_{s} \\
r M_{s \theta} \\
r N_{r} \\
r N_{z} \\
r N_{s \theta}
\end{array}\right]^{(n)}
$$

Tenth-Order Matrix Form of the Shell Equations:

$$
-\frac{d}{d s}\left[\begin{array}{l}
\mathbf{F} \\
\mathbf{D}
\end{array}\right]+[\mathbf{A}(s)]\left[\begin{array}{l}
\mathbf{F} \\
\mathbf{D}
\end{array}\right]=\left[\begin{array}{l}
\mathbf{P} \\
\mathbf{0}
\end{array}\right]
$$

The equations include the effects of

- Transverse shear deformation

- Moderate rotation: $\varepsilon^{(N L)}=\varepsilon^{(L)}+\frac{1}{2} \beta^{2}$

- Initial prestress: Perturbations relative to a nominal state of membrane tension are considered. 


\section{$\underline{\text { Response of a Shell to Edge Loads }}$}

I. Membrane (Negligible Bending):

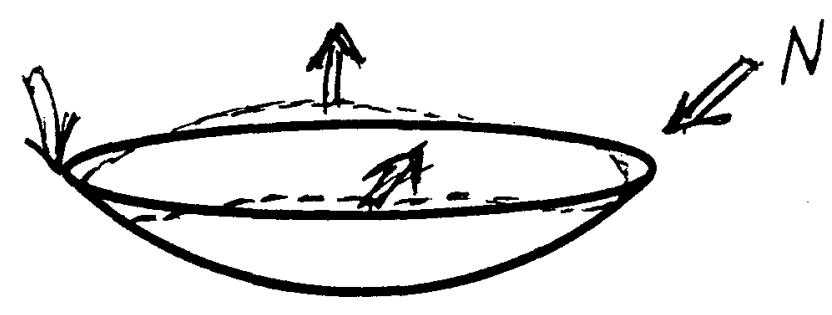

II. Inextensional (Negligible Membrane Strain):

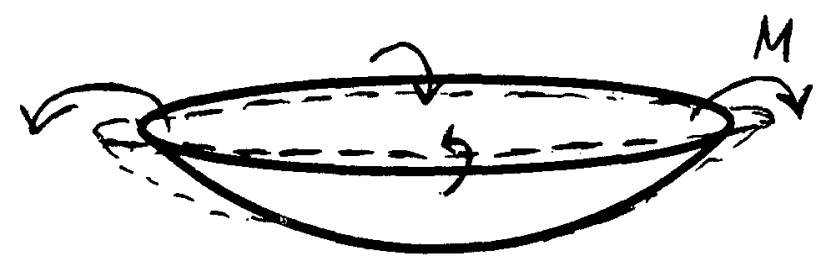

III. Edge Effects (Coupled Stretching/Bending):

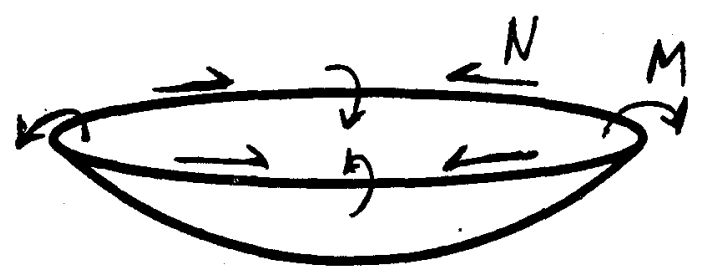




\section{$\underline{\text { Asymptotic Solutions }}$}

For the tenth-order system:

$$
-\frac{d \mathbf{Y}}{d s}+\mathbf{A}(s) \mathbf{Y}=\mathbf{0}
$$

Use the formal asymptotic expansion:

$$
\begin{aligned}
\mathbf{Y}= & \exp \left(\int \xi(s) d s\right)\left[\mathbf{Y}_{0}(s)+\mathbf{Y}_{1}(s)+\ldots\right] \\
& (\mathbf{A}-\mathbf{I} \xi) \mathbf{Y}_{0}=\mathbf{0} \\
& (\mathbf{A}-\mathbf{I} \xi) \mathbf{Y}_{i}=\frac{d \mathbf{Y}_{i-1}}{d s} \quad(i \geq 1)
\end{aligned}
$$

Note: This approach is accurate for the edgeeffect solutions, but not for the membrane and inextensional solutions. 


\section{Decay Distances of the EDGE EFFECTS}

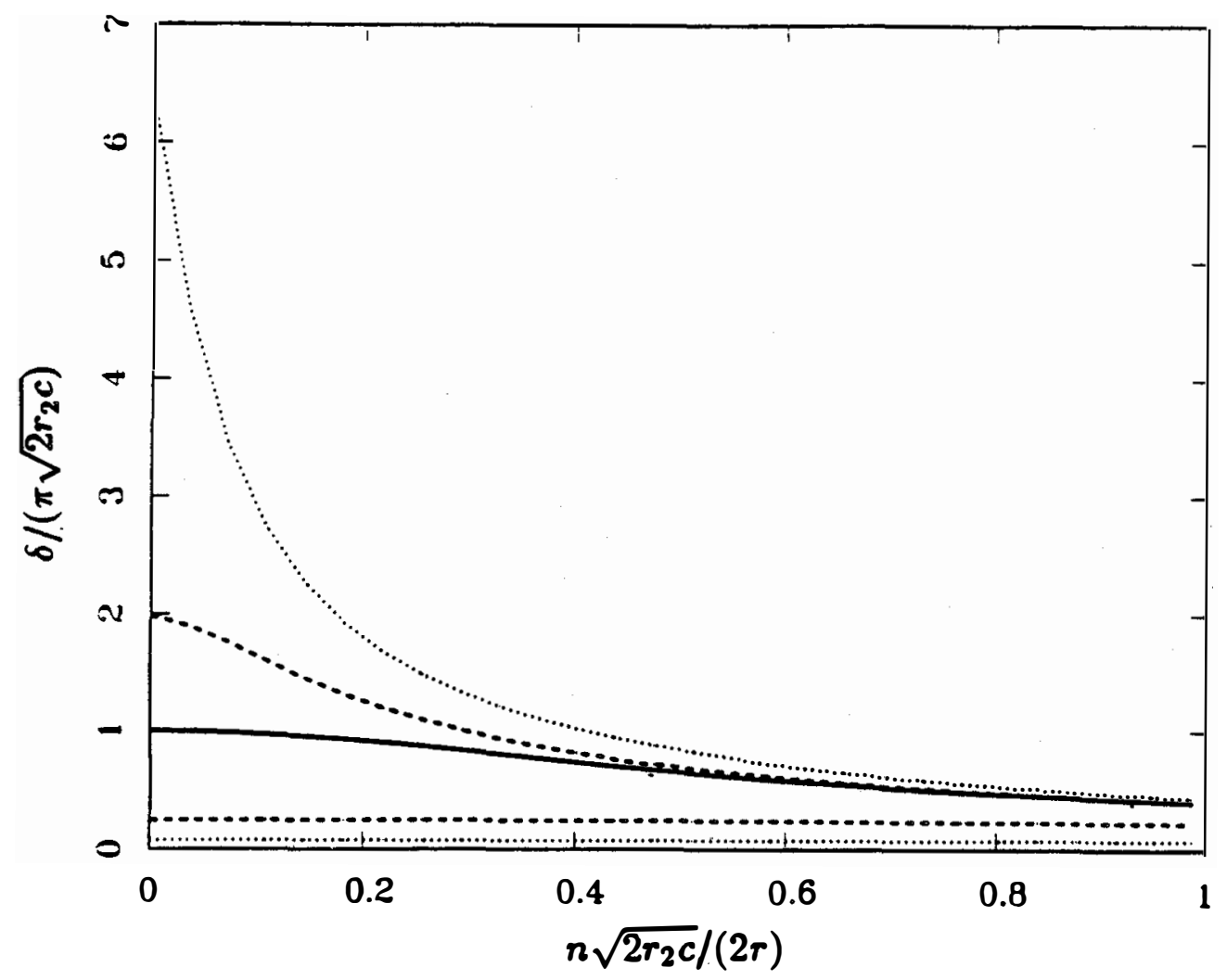

Decay distance $\delta$ as a function of $n$ for the two edge bending solutions, with nominal prestress $N_{0} r_{2} /(2 E t c)=$ $N_{o r} /(2 E t c)=0$ (solid line), 4 (dashed line), and 40 (dotted line). The longest decay distance occurs for the axisymmetric case $n=0$. 


\section{$\underline{\text { Decay Behavior of the Edge Effects }}$}

For low membrane prestress; $\rho=\frac{N R}{2 E t c}=O(1)$ :

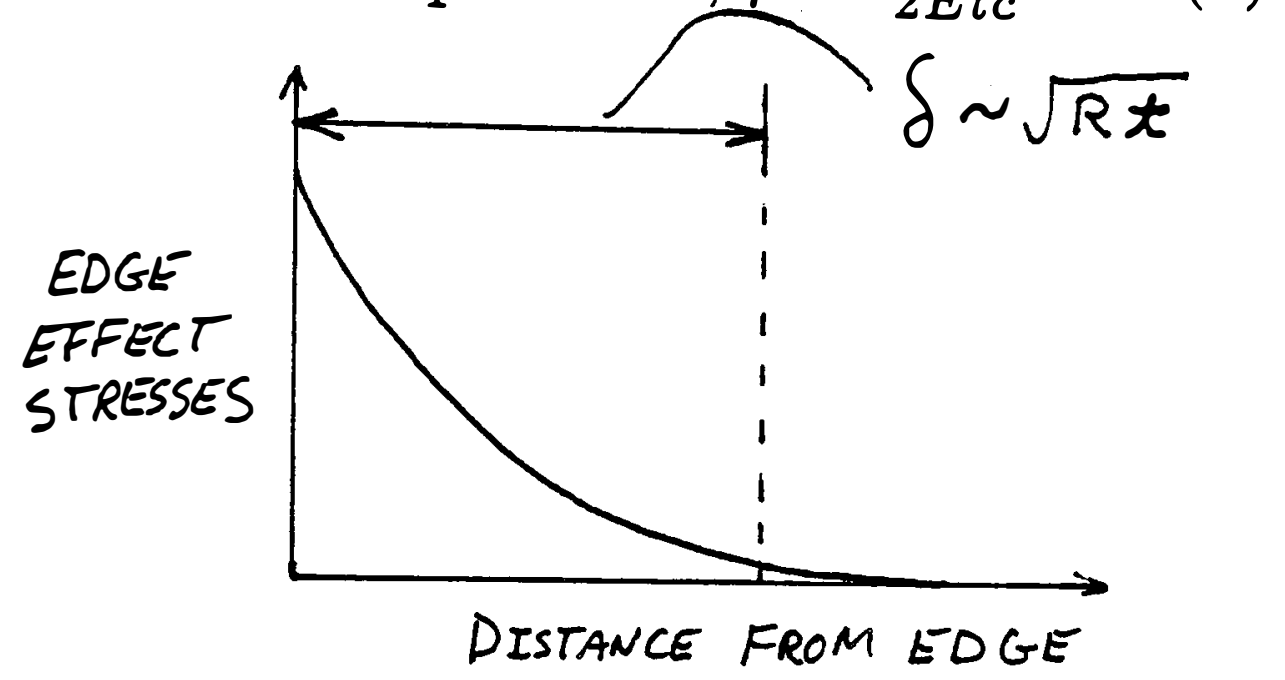

For high membrane prestress; $\rho=\frac{N R}{2 E t c} \gg 1$ :

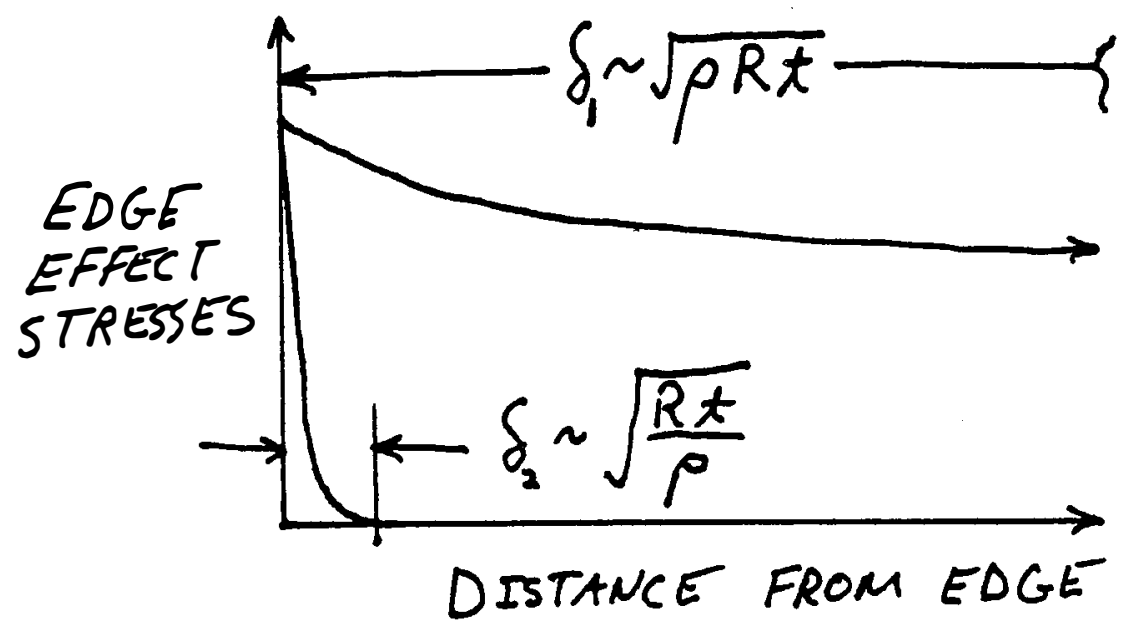

(two edge-effect solutions) 


\section{The Shallow Shell Equations}

For a paraboloidal dish, the equations are

$$
\begin{gathered}
\frac{1}{E t} \Delta \Delta \phi+\frac{1}{R} \Delta u_{z}=0 \\
\operatorname{Etc}^{2} \Delta \Delta u_{z}-\bar{N} \Delta u_{z}-\frac{1}{R} \Delta \phi=0
\end{gathered}
$$

Membrane and inextensional solutions to the above equations take the form of simple harmonic functions which satisfy

$$
\begin{aligned}
\Delta \phi & =0 \\
\Delta u_{z} & =0
\end{aligned}
$$




\section{Description of the DISH Computer Program}

This appendix provides a brief description of the DISH computer program for the analysis. of paraboloidal shells. The program is written in standard FORTRAN-77 and can be run on an IBM PC. A sample input file is shown in the following table.

Table C-1. Sample input file for the DISH program.

Input File DISH.IN, for the DISH structural analysis program.

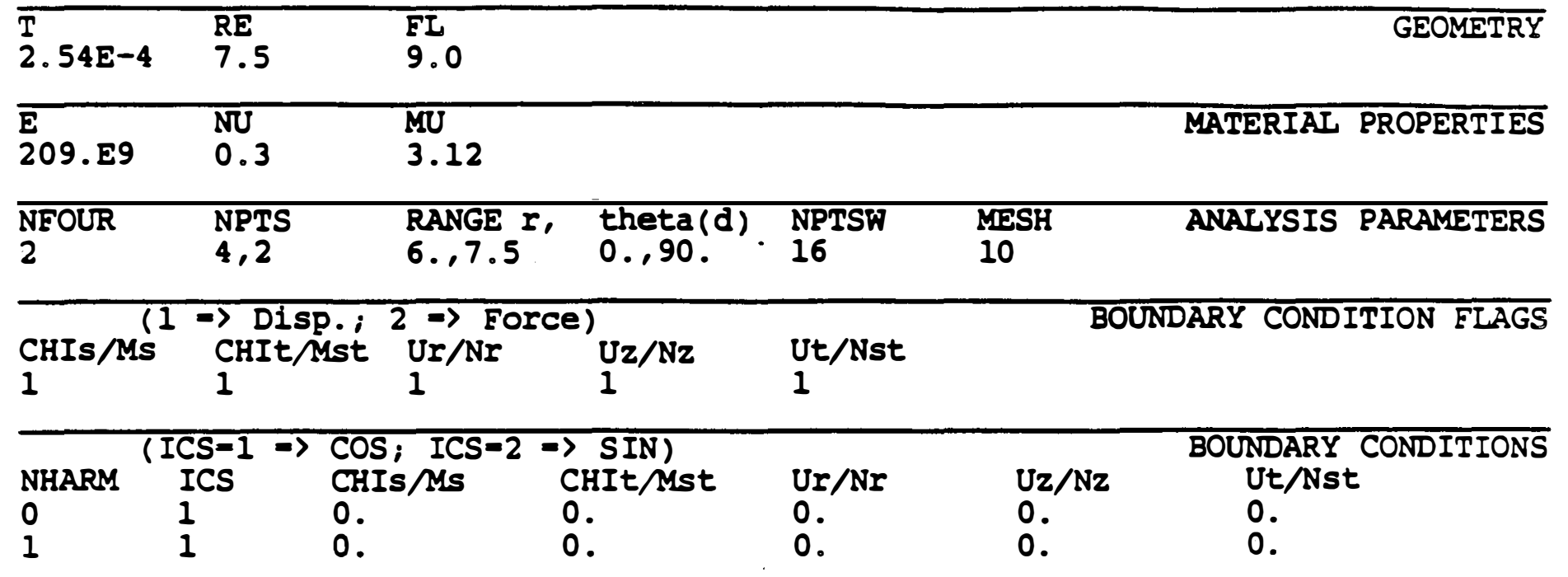

PNOM

PRESSURE LOAD DATA

2000.

(WINDAT(I), I=1,NPTSW)

$\begin{array}{lllll}0 . & 0.277778 \mathrm{E}+02 & 0.555556 \mathrm{E}+02 & 0.833333 \mathrm{E}+02 & 0.111111 \mathrm{E}+03 \\ 0.138889 \mathrm{E}+03 & 0.166667 \mathrm{E}+03 & 0.194444 \mathrm{E}+03 & 0.222222 \mathrm{E}+03 & 0.250000 \mathrm{E}+03 \\ 0.277778 \mathrm{E}+03 & 0.305556 \mathrm{E}+03 & 0.333333 \mathrm{E}+03 & 0.361111 \mathrm{E}+03 & 0.388889 \mathrm{E}+03 \\ 0.416667 \mathrm{E}+03 & & & & \end{array}$

Table C-1 shows an input file for the analysis of a paraboloid with clamped edges and wind loading. The first line of numbers contains the thickness $T$, edge radius $R E$, and focal length FL. The second line of numbers contains the elastic modulus E, Poisson's ratio NU, and the shear flexibility factor MU (see Eq. (5)). 
Specifications for paraboloidal dish.

\begin{tabular}{|l|c|c|}
\hline \multicolumn{1}{|c|}{ Geometry } & Material Properties & Load \\
\hline$t=0.254 \mathrm{~mm}$ & $E=209 \mathrm{GPa}$ & $p=2000 \mathrm{~Pa}$ \\
$r_{e}=7.5 \mathrm{~m}$ & $\nu=0.3$ & \\
$f=9 \mathrm{~m}$ & & \\
\hline
\end{tabular}


Prescribed Radial Edge Deformation: $u_{r}=U_{0} \cos 2 \theta$
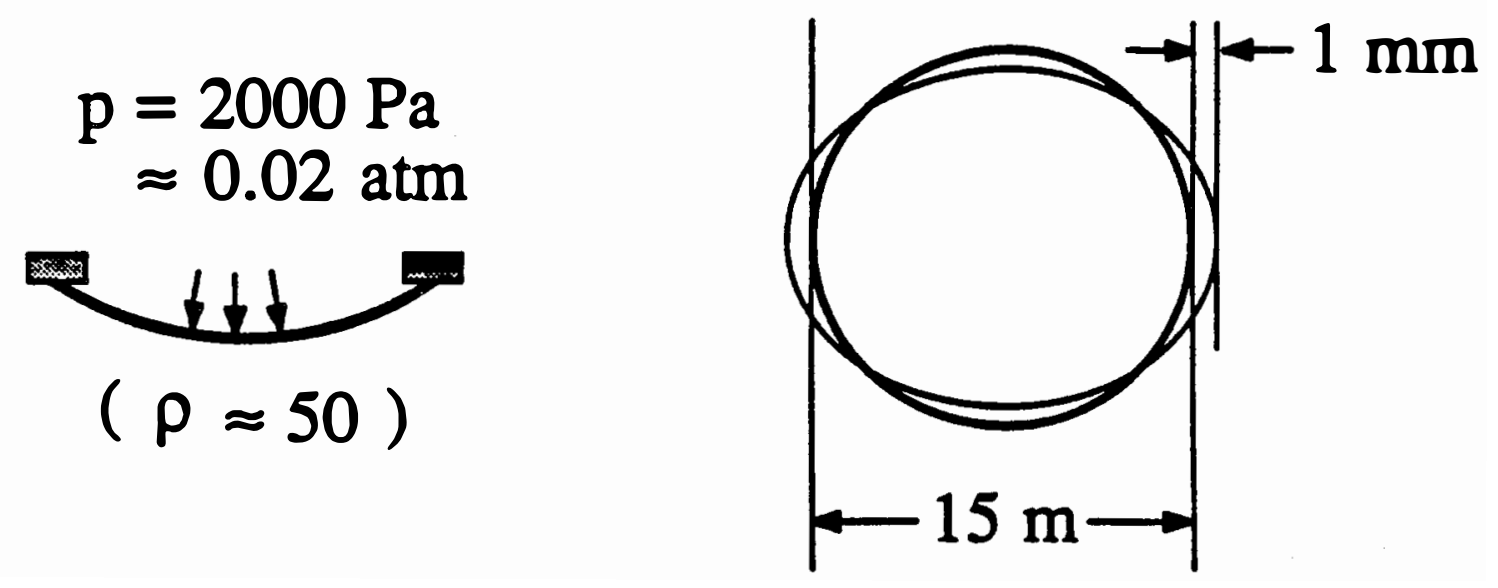

ROTATSONS $\left.x_{s}\right)$
$x_{\theta}$

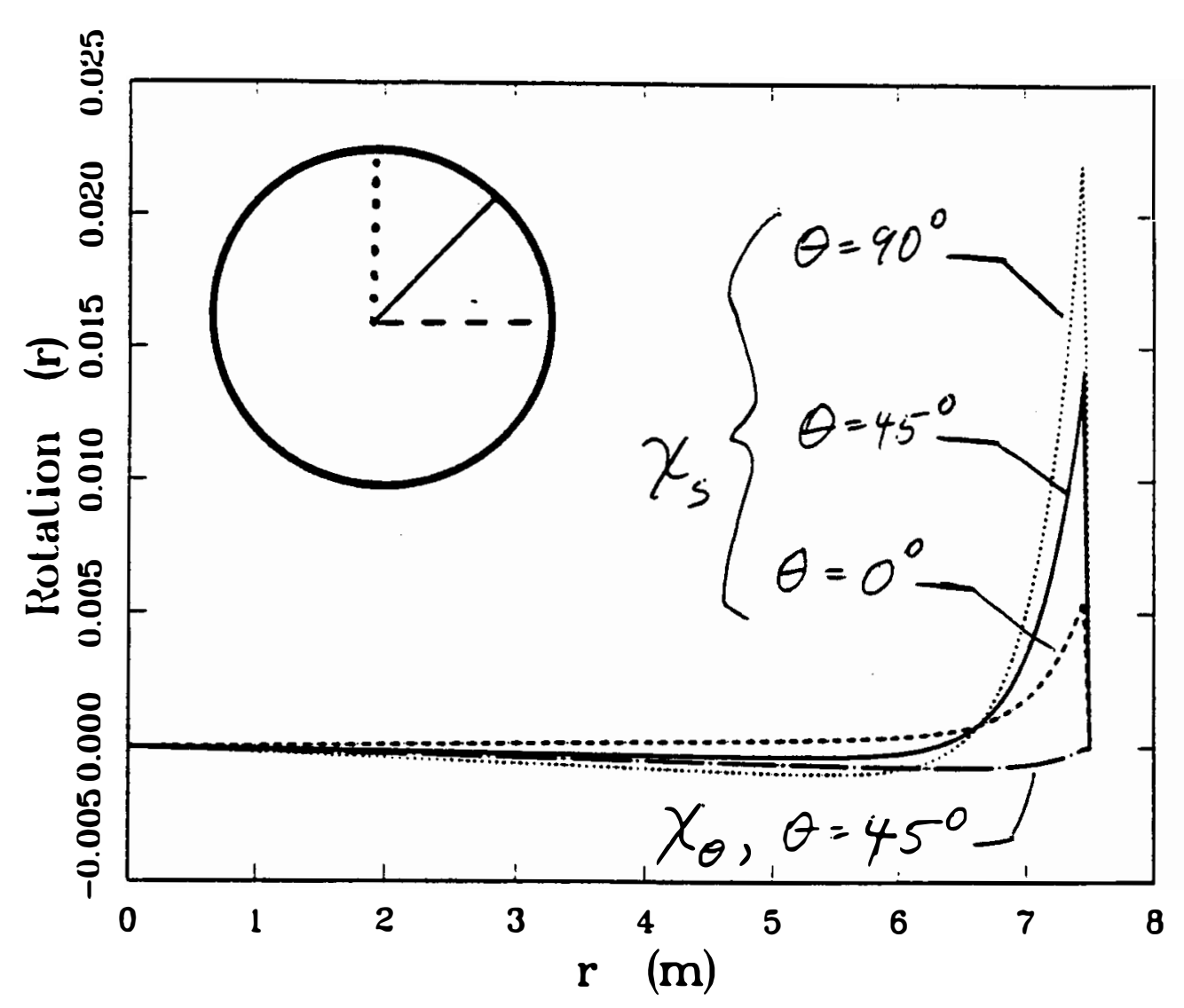




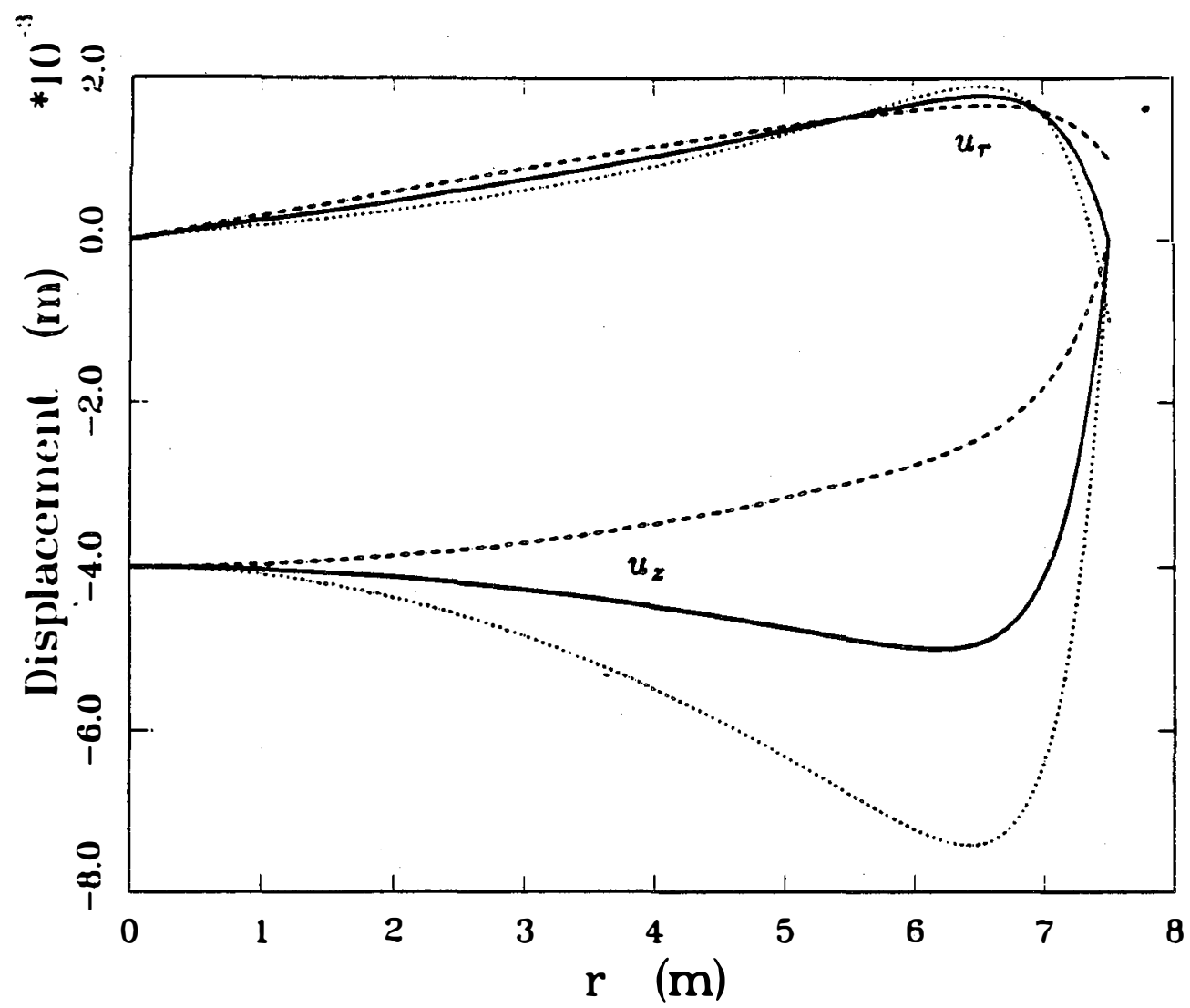

Radial displacement $u_{r}$ and vertical displacement $u_{z}$ as functions of $r$ for a paraboloidal shell subjected to a $\cos 2 \theta$ prescribed radial edge displacement. Results shown for $\theta=0^{\circ}$ (dashed lines) and $\theta=90^{\circ}$ (dotted lines). The results for the axisymmetric case of a clamped edge are shown for comparison (solid lines). 


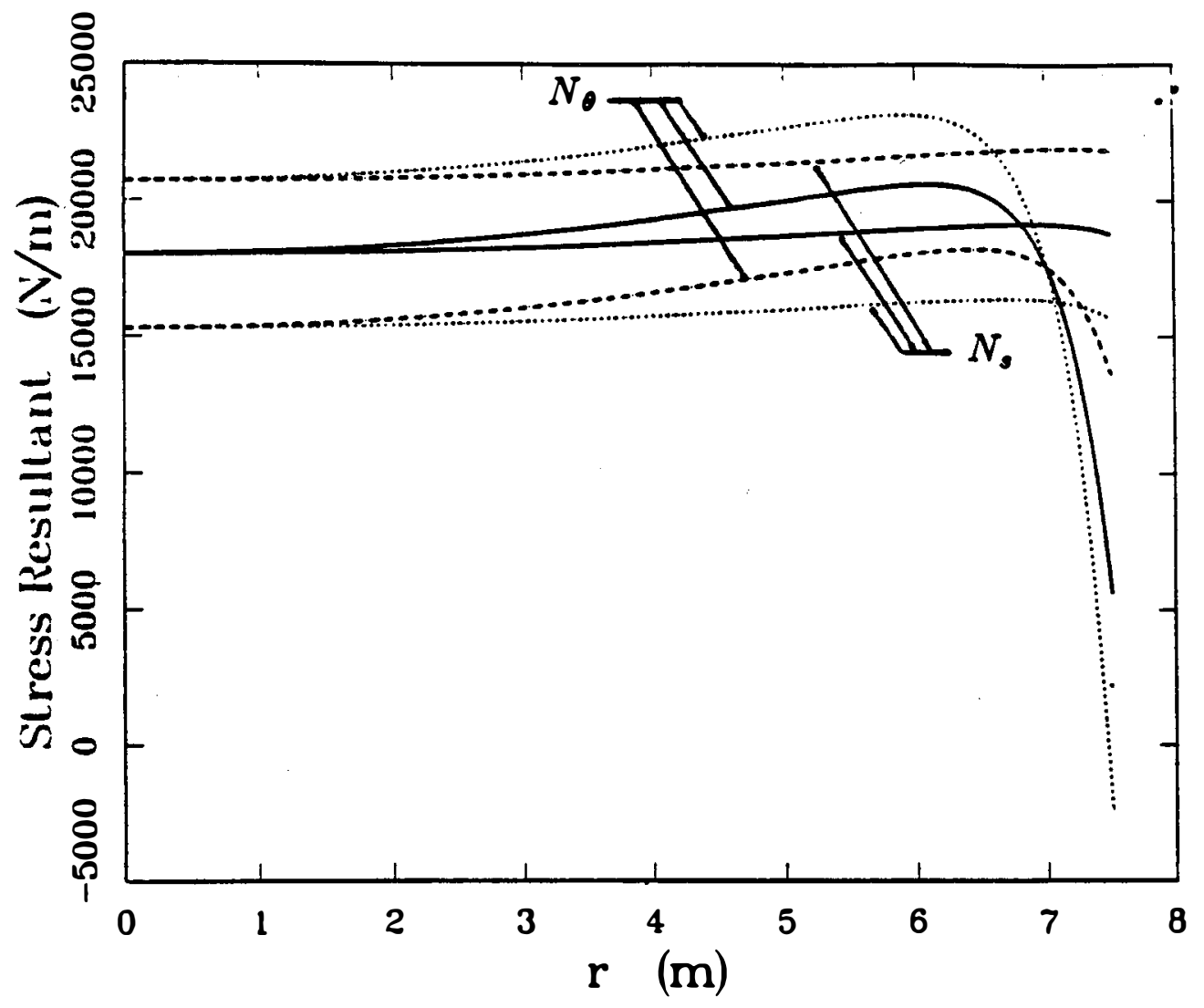

Stress resultants $N_{\text {, and }} N_{\theta}$ as functions of $r$ for a paraboloidal shell subjected to a $\cos 2 \theta$ prescribed radial edge displacement. The stress resultants are shown for $\theta=$ $0^{\circ}$ (dashed lines) and $\theta=90^{\circ}$ (dotted lines), and for the acismmetric case of a clamped edge for comparison (solid lines). Note the significant drop in the circumferential stress $N_{0}$ near the edge for $\theta=90^{\circ}$. 


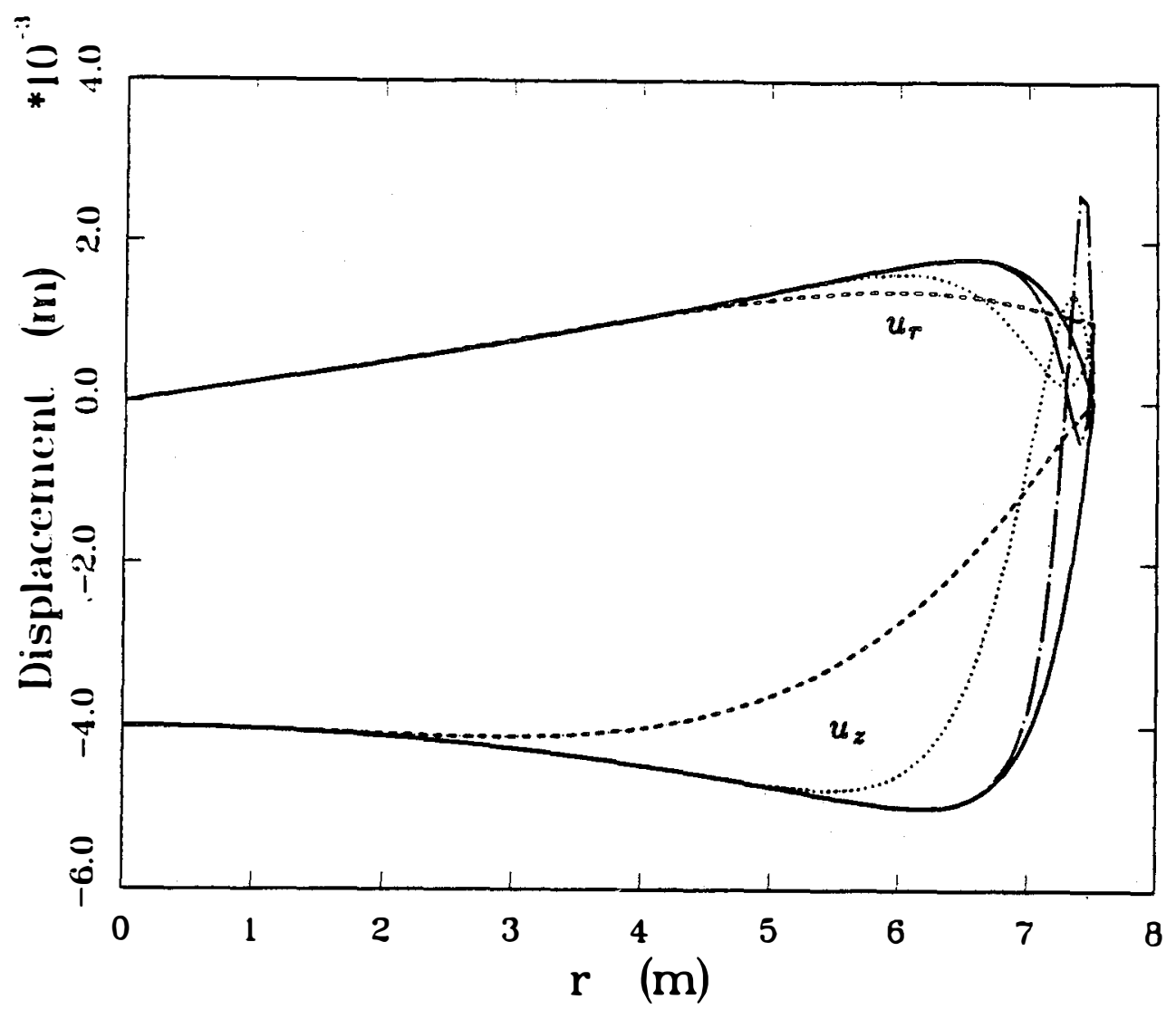

Radial displacement $u_{r}$ and vertical displacement $u_{z}$ along the line $\theta=0^{\circ}$ for a paraboloidal shell subjected to $\cos n \theta$ prescribed radial edge displacements. Results shown for $n=4$ (dashed lines), $n=16$ (dotted lines), and $n=64$ (dot-dashed lines). The results for the axisymmetric case of a clamped edge are shown for comparison (solid lines). With increasing $n$ the edge effects decay more rapidly; outside the edge zone, the displacements approach those of the axisymmetric case. 
Asymmetric Wind Load

$\mathrm{p}=2000 \mathrm{~Pa}$

$+1+1$

Axisymmetric

$(\mathrm{n}=0)$
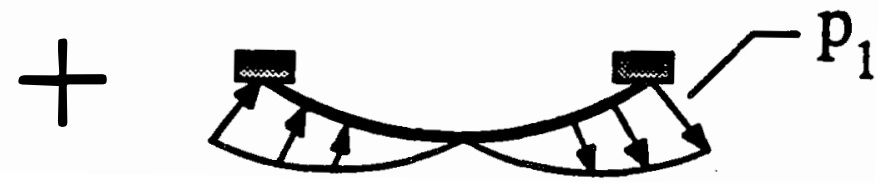

$\cos \theta$

$(\mathrm{n}=1)$

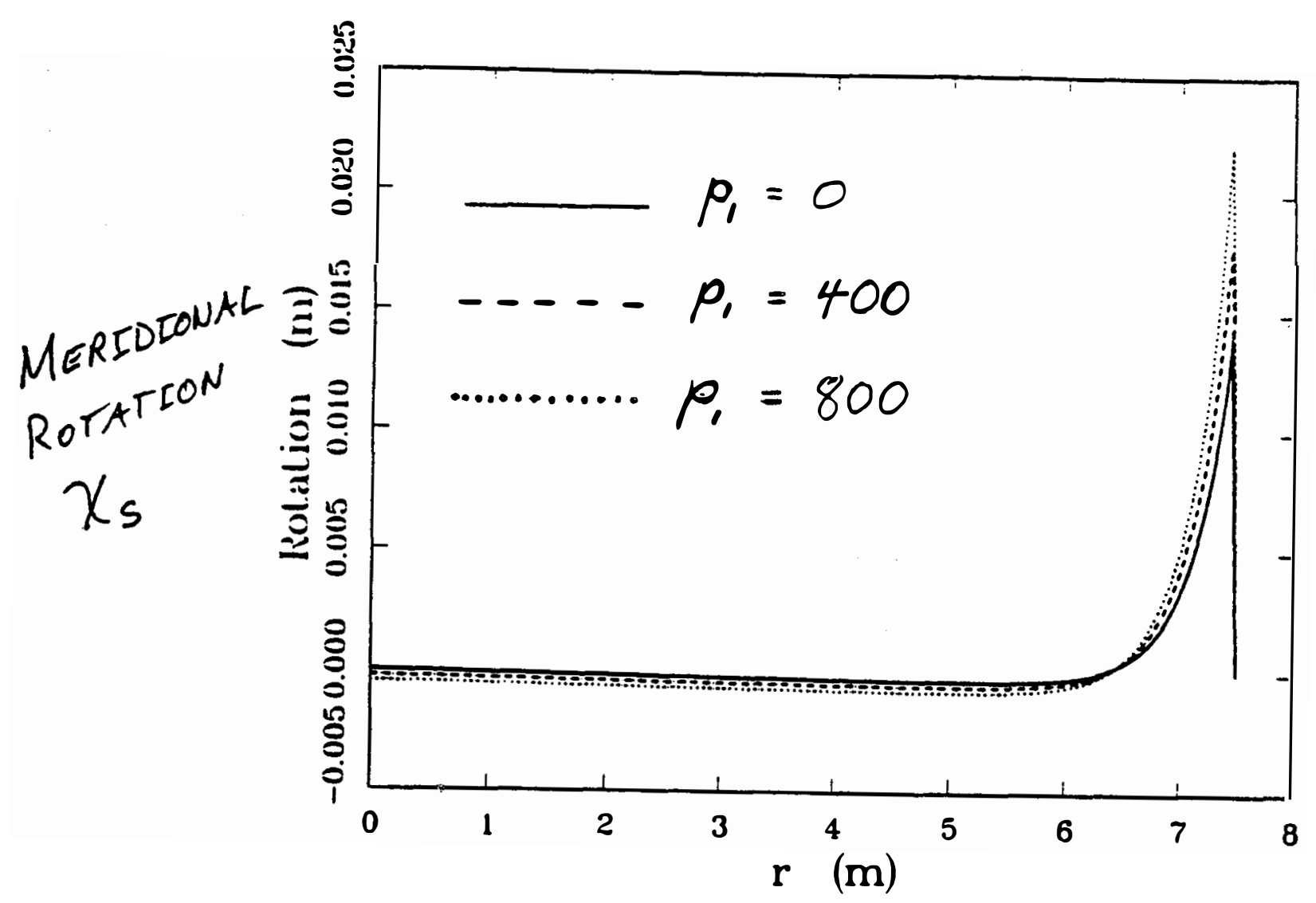




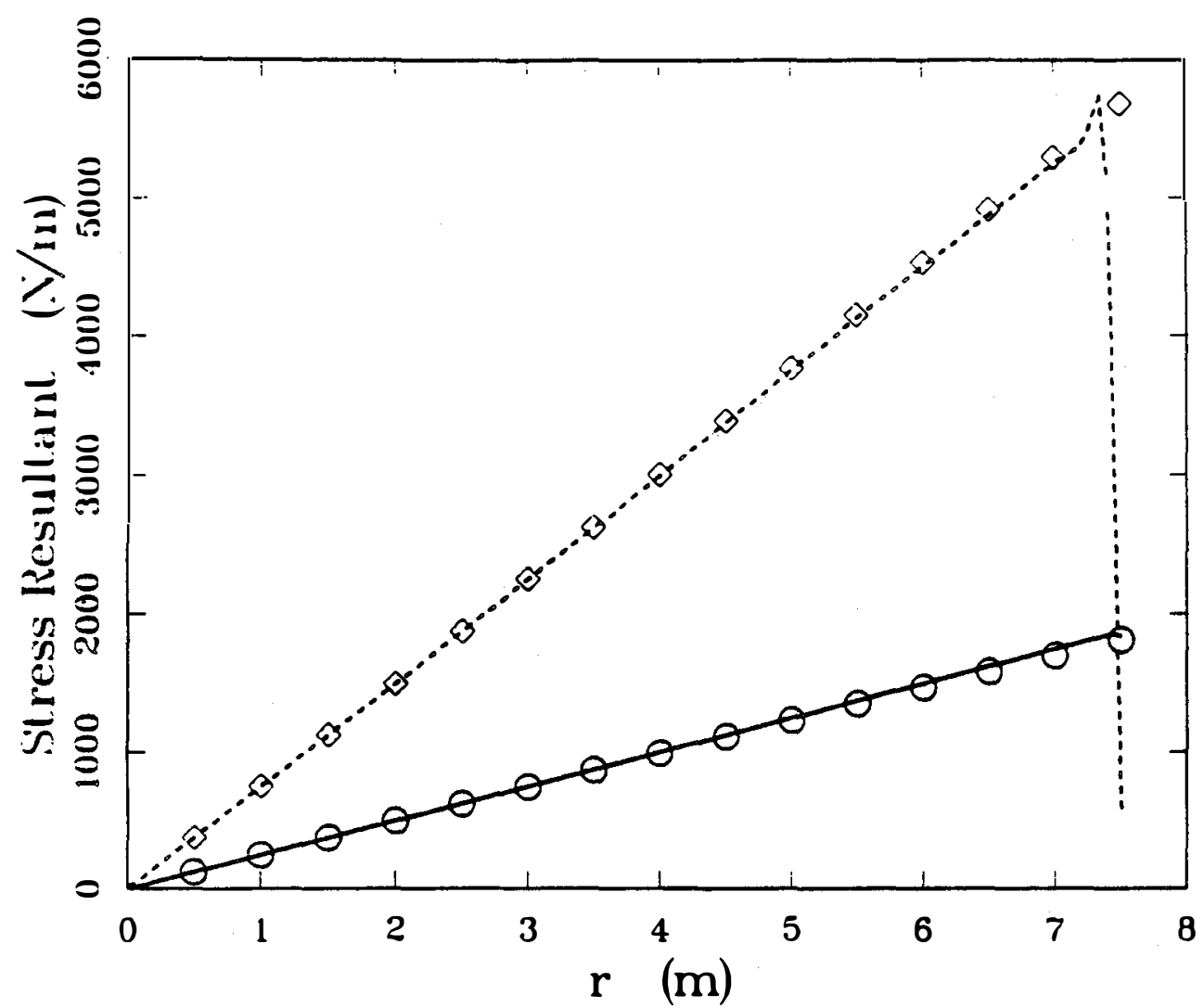

Stress resultants $N_{\theta}$ (solid line) and $N_{\theta}$ (dashed line) along $\theta=0^{\circ}$ for a clamped paraboloidal shell subjected to the asymmetric load $p_{n}=p_{w} \sin \phi \cos \theta$, with $p_{w}=1000 \mathrm{~Pa}$ and a nominal pressure load of $2000 \mathrm{~Pa}$. The circles and diamonds are the membrane solution from Flügge (1973), which agrees with the present analysis except near the edge. The present analysis includes the rapidly decaying edge bending effects, which account for the abrupt drop in the circumferential stress $N_{0}$ near the edge. 


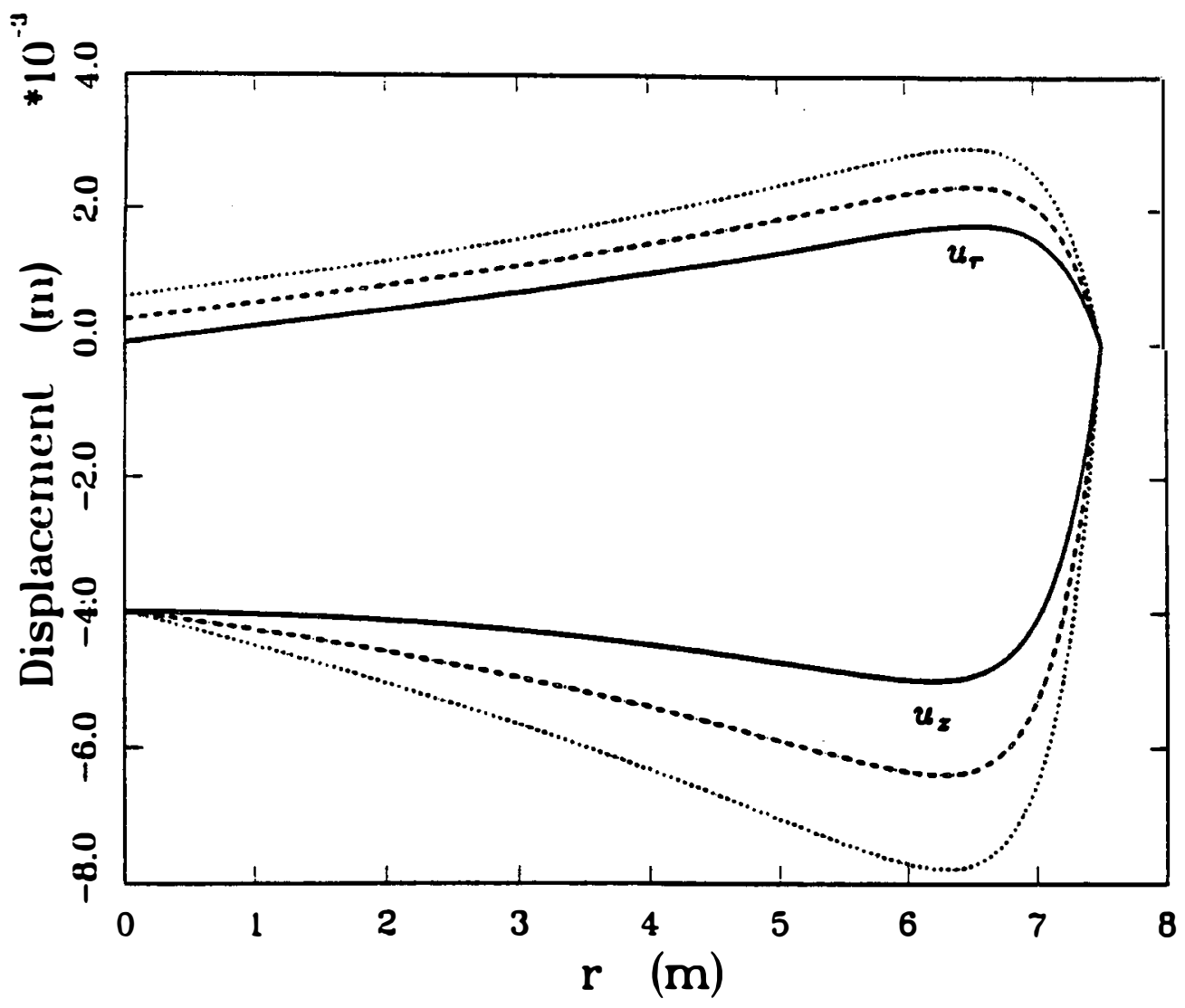

Displacements $u_{r}$ and $u_{z}$ along $\theta=0^{\circ}$ for a paraboloidal shell with a clamped edge subjected to the asymmetric load $p_{n}=p_{w} \sin \phi \cos \theta$, for $p_{w}=1000 \mathrm{~Pa}$ (dashed lines) and $p_{w}=2000 \mathrm{~Pa}$ (dotted lines). The displacements of the axisymmetric solution with the nominal pressure load of $2000 \mathrm{~Pa}$ are shown for comparison (solid lines). 


\section{Concluding Remarks}

- The equations for non-axisymmetric deformations of shells of revolution have been derived in state-vector (matrix) form, including the effects of initial prestress due to pressue load, and transverse shear deformation.

- Asymptotic solutions for non-axisymmetic edge bending effects are presented. These effects involve coupled bending and membrane behavior, and typically decay rapidly with increasing distance from the edge. High prestress is found to affect the decay behavior significantly.

- For the non-axisymmetic membrane and inextensional behavior of a paraboloidal shell, solutions are obtained using shallow shell theory.

- A computer program has been developed which is based on the asymptotic solutions and the results from shallow shell theory. The program can be used for axisymmetric and non-axisymmetric analyses of a paraboloidal dish, and runs on a PC. 


\section{Appendix C}

\section{Structural and Structural/Optical Analysis of Dish Membranes*}

presented at SERI on August 25, 1988

by Chad D. Balch

*work supported by SERI under subcontract number CJ-8-00632-1, $6 / 20 / 88$ through $8 / 26 / 88$. 


\section{OUTLINE}

Part I: Structural Analysis

A. Overview of capabilities and limitations of the structural analysis sof tware

B. Theoretical background

C. The DISH structural code and related processors, examples

D. Interface with a finite element model

Part II: Coupled Structural/Optical Analysis

A. Interface with the OPTDSH ray trace code

B. The OPTDSH ray trace code (Jorgensen)

C. Coupled structural/optical analysis; examples and software demo.

Wrap up with:

Work in Progress; Concluding Remarks 


\section{The DISH Program: Capabilities}

1. Based on analytical solutions from shell theory; runs in a few minutes on a PC.

2. Handles the following problems:

A. Arbitrary edge deformation (under pressure loading)

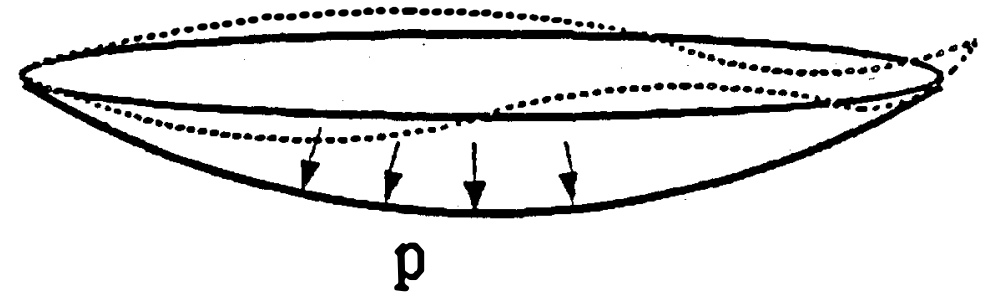

B. Asymmetric pressure loading

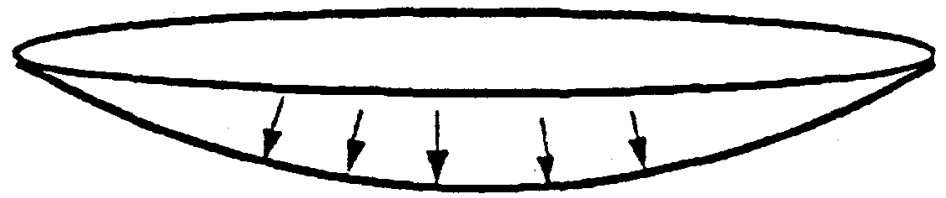

$\mathrm{p}$

$0+$

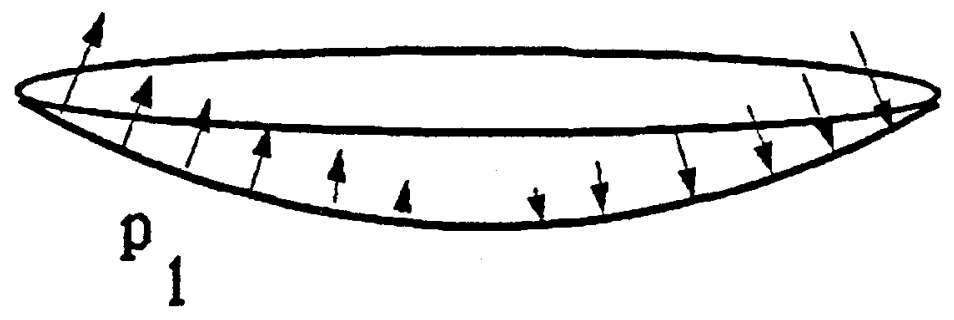

3. Interfaces with OPTDSH for rapid ray trace analysis. 


\section{Limitations}

1. Thin shells

2. Small strains

3. Small perturbations from the "membrane solution"

4. Edge effects should die out with decay distances small relative to radii of curvature

5. Edge angle should be less than about $30^{\circ}$, since some shallow shell solutions are used. 
Non-axisymmetric Problems: Kinematics
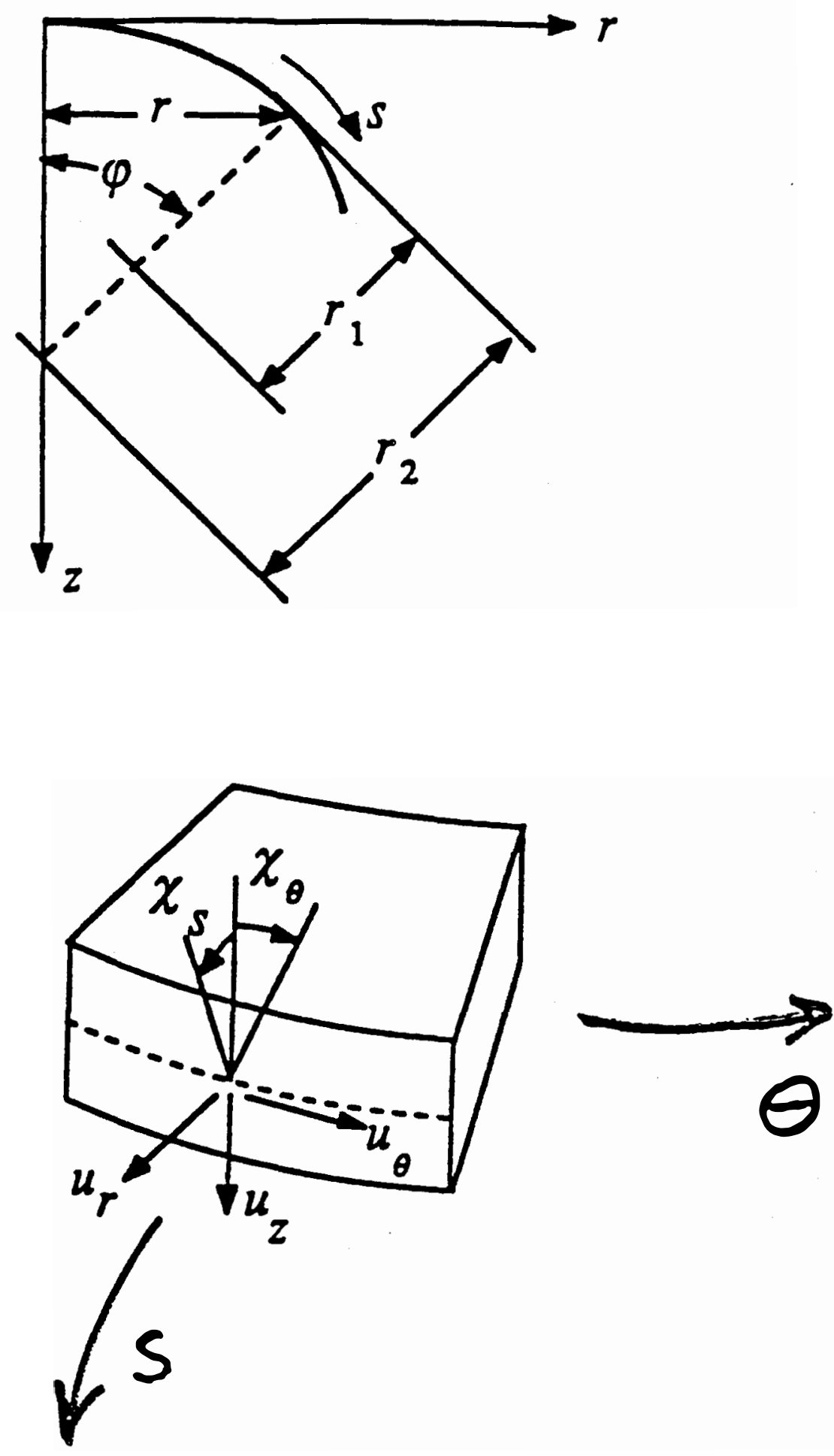
$\underline{\text { Non-axisymmetric Problems: Governing Equations }}$

Displacement and Force Quantities:

$$
\mathbf{D}=\left[\begin{array}{c}
\chi_{s} \\
\chi_{\theta} \\
u_{r} \\
u_{z} \\
u_{\theta}
\end{array}\right]^{(n)} \quad ; \quad \mathbf{F}=\left[\begin{array}{c}
r M_{s} \\
r M_{s \theta} \\
r N_{r} \\
r N_{z} \\
r N_{s \theta}
\end{array}\right]^{(n)}
$$

Tenth-Order Matrix Form of the Shell Equations:

$$
-\frac{d}{d s}\left[\begin{array}{l}
\mathrm{F} \\
\mathrm{D}
\end{array}\right]^{(n)}+[
$$

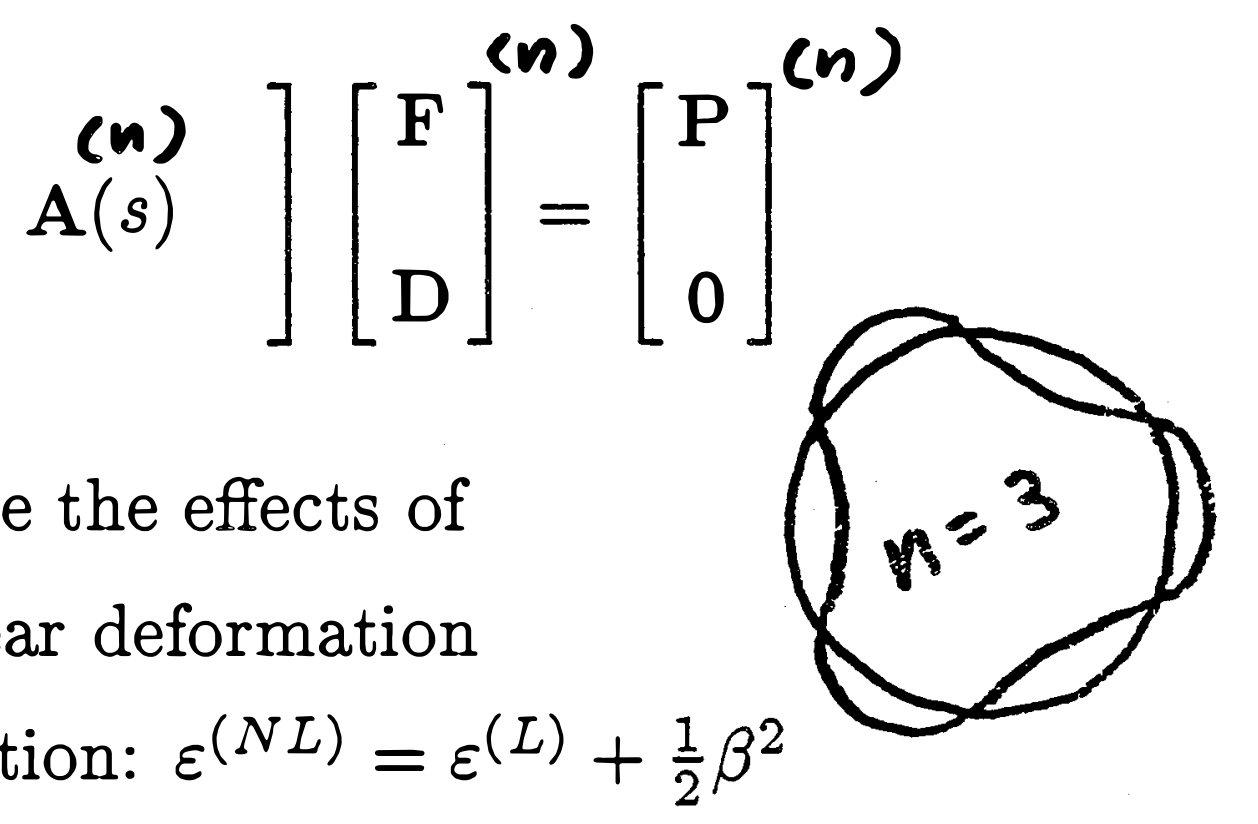

The equations include the effects of

- Transverse shear deformation

- Moderate rotation: $\varepsilon^{(N L)}=\varepsilon^{(L)}+\frac{1}{2} \beta^{2}$

- Initial prestress: Perturbations relative to a nominal state of membrane tension are considered. 


\section{Asymptotic Solutions}

For the tenth-order system:

$$
-\frac{d \mathbf{Y}}{d s}+\mathbf{A}(s) \mathbf{Y}=0
$$

Use the formal asymptotic expansion:

$$
\begin{aligned}
\mathbf{Y}= & \exp \left(\int \xi(s) d s\right)\left[\mathbf{Y}_{0}(s)+\mathbf{Y}_{1}(s)+\ldots\right] \\
& (\mathbf{A}-\mathbf{I} \xi) \mathbf{Y}_{0}=0 \\
& (\mathbf{A}-\mathbf{I} \xi) \mathbf{Y}_{i}=\frac{d \dot{Y}_{i-1}}{d s} \quad(i \geq 1)
\end{aligned}
$$

Note: This approach is accurate for the edgeeffect solutions, but not for the membrane and inextensional solutions. (For present formulation) 


\section{$\underline{\text { Response of a Shell to Edge Loads }}$}

I. Membrane (Negligible Bending):

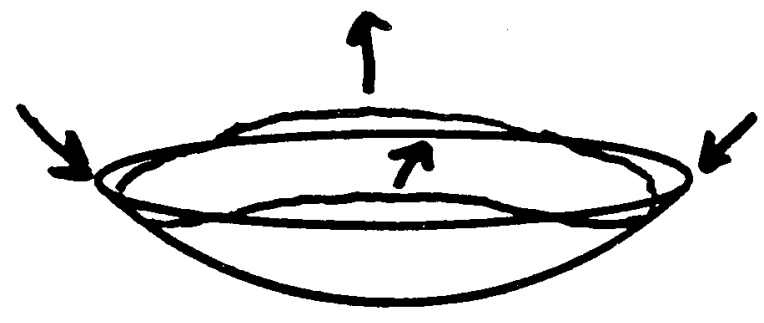

II. Inextensional (Negligible Membrane Strain):

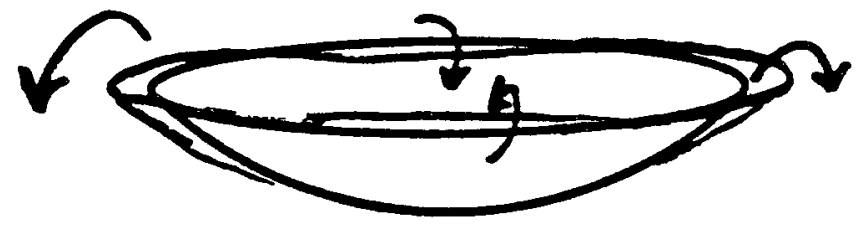

III. Edge Effects (Coupled Stretching/Bending):

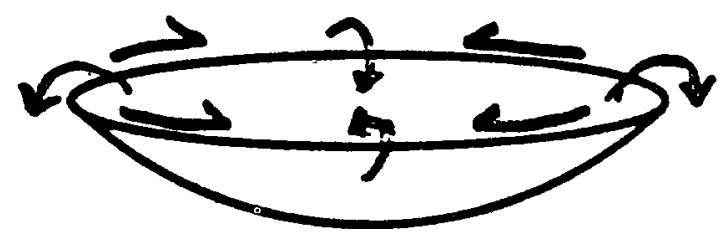




\section{Decay Behavior of the Edge Effects}

For low membrane prestress; $\rho=\frac{N R}{2 E t c}=O(1)$ :

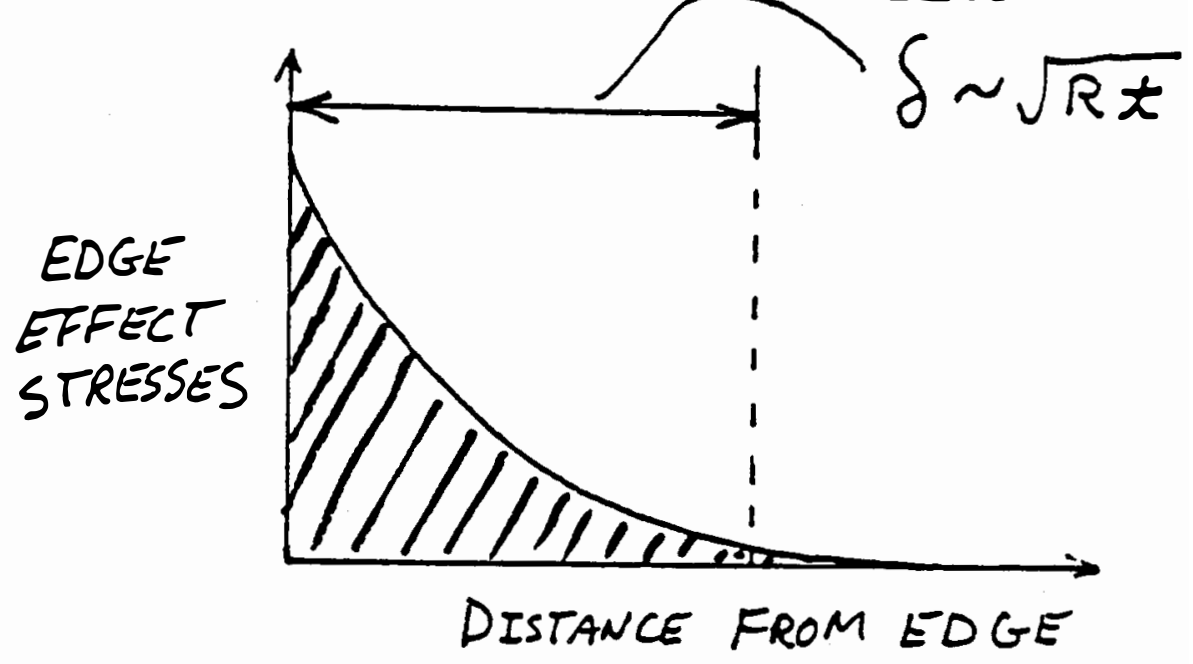

For $\underline{\text { high }}$ membrane prestress; $\rho=\frac{N R}{2 E t c} \gg 1$ :

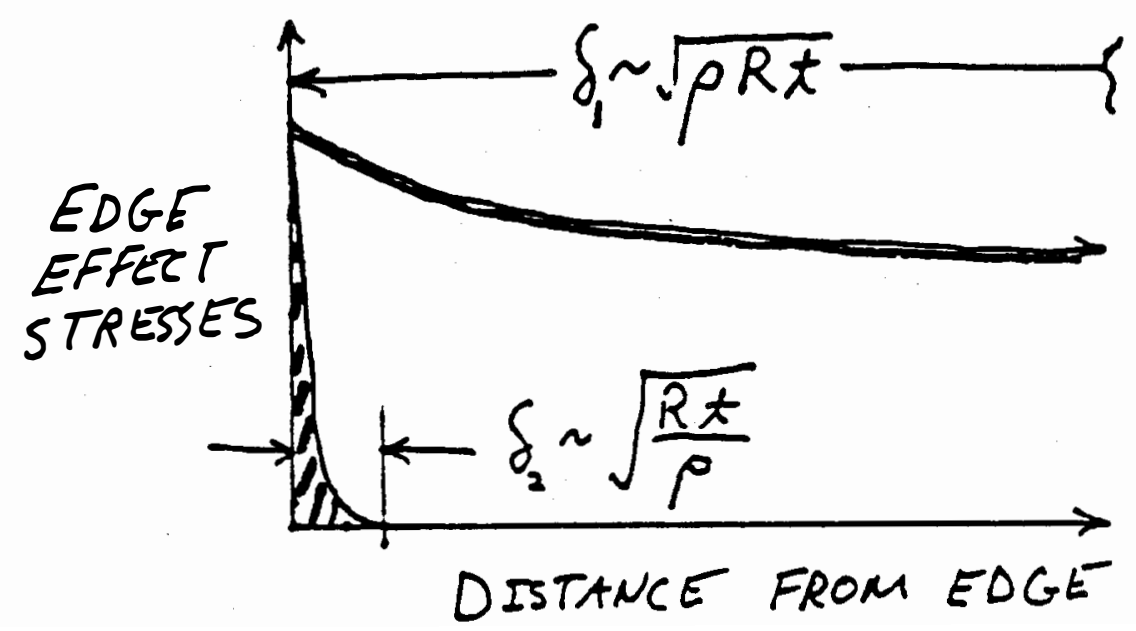

(two edge-effect solutions) 


\section{The Shallow Shell Equations}

For a paraboloidal dish, the equations are

$$
\begin{gathered}
\frac{1}{E t} \Delta \Delta \phi+\frac{1}{R} \Delta u_{z}=0 \\
\operatorname{Etc}^{2} \Delta \Delta u_{z}-\bar{N} \Delta u_{z}-\frac{1}{R} \Delta \phi=0
\end{gathered}
$$

Membrane and inextensional solutions to the above equations take the form of simple harmonic functions which satisfy

$$
\begin{aligned}
\Delta \phi & =0 \\
\Delta u_{z} & =0
\end{aligned}
$$


PROGRAM

FILE

i⿱
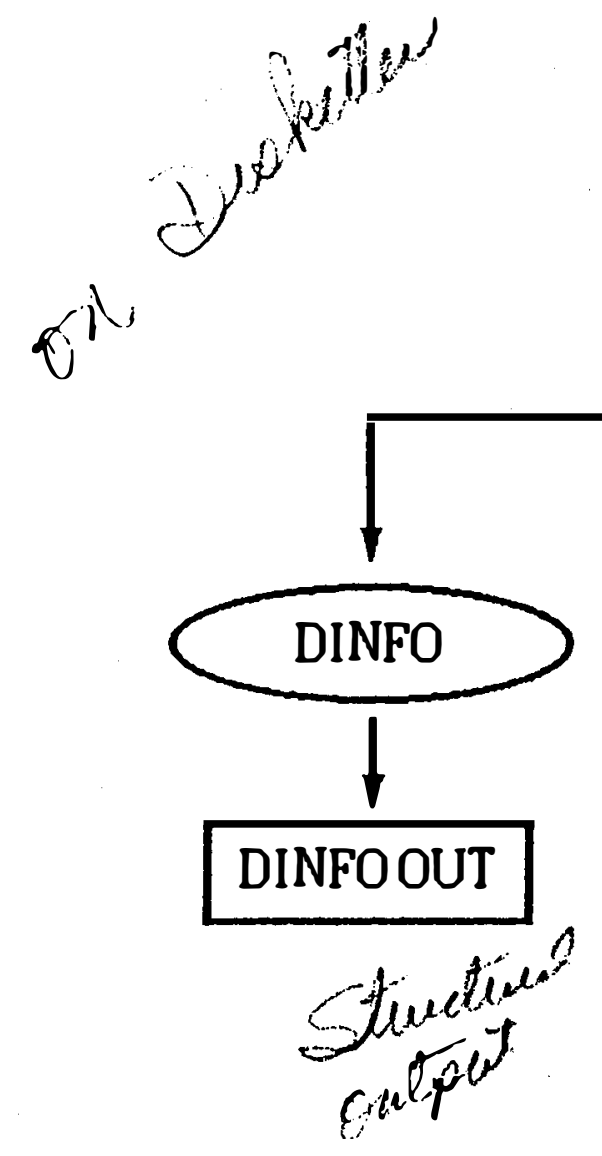

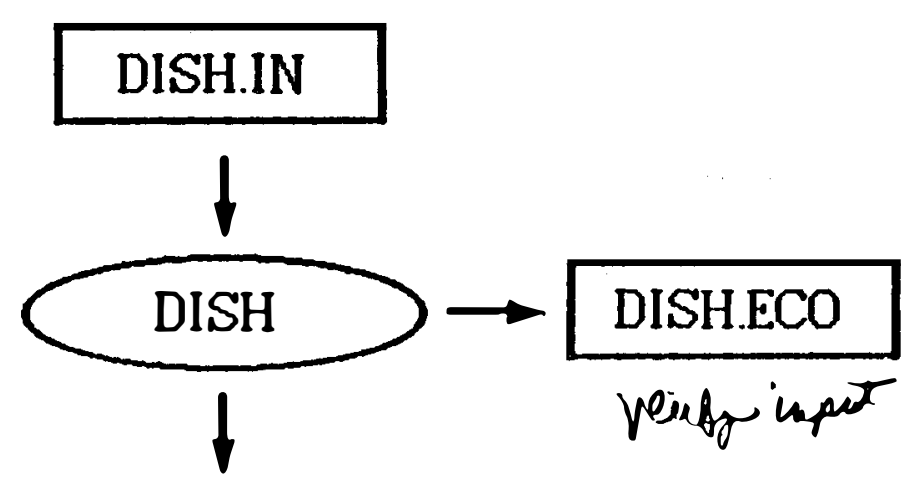

$\frac{\text { DISH.DAT }}{\text { Qupdedato into }}$
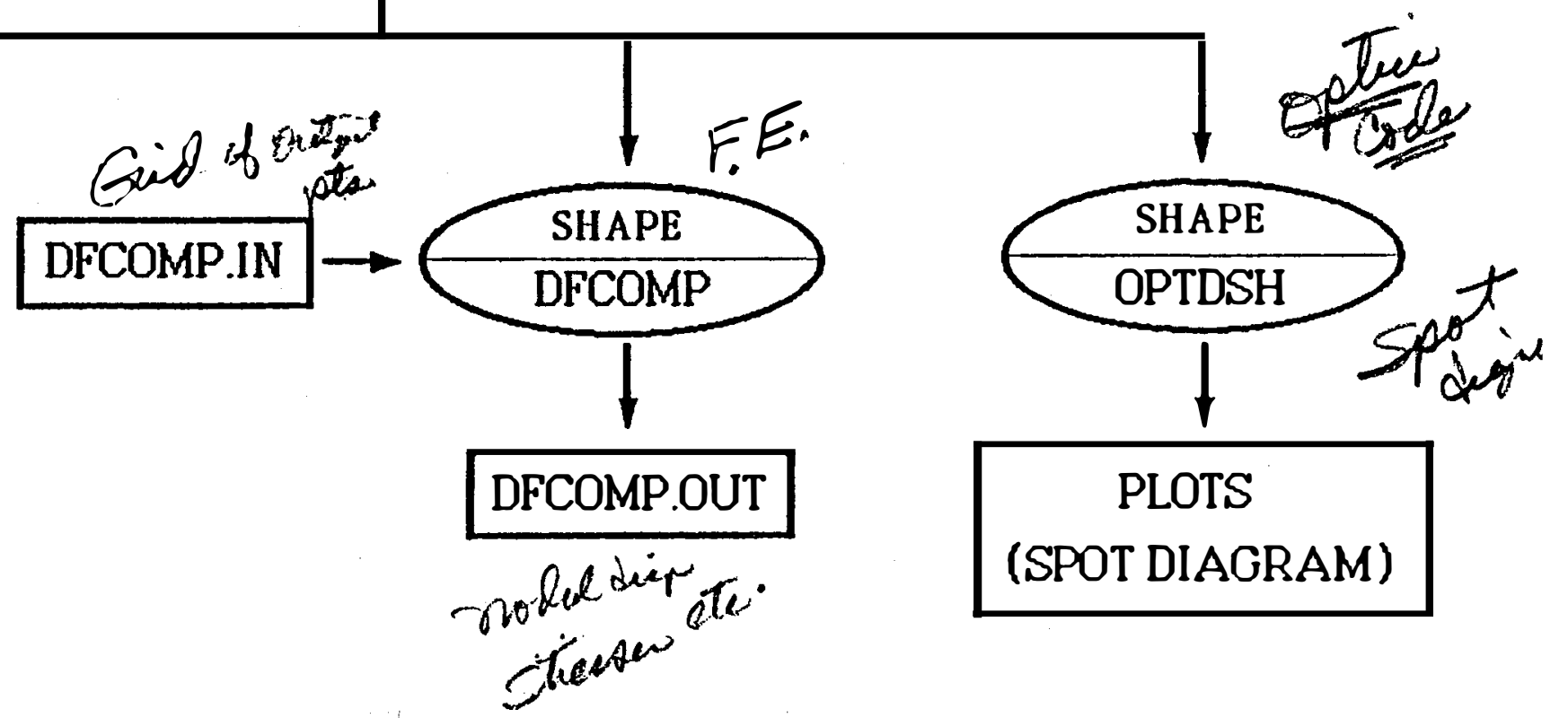

Figure 1: Flowchart of data paths for structural and structural/optical analyses. 


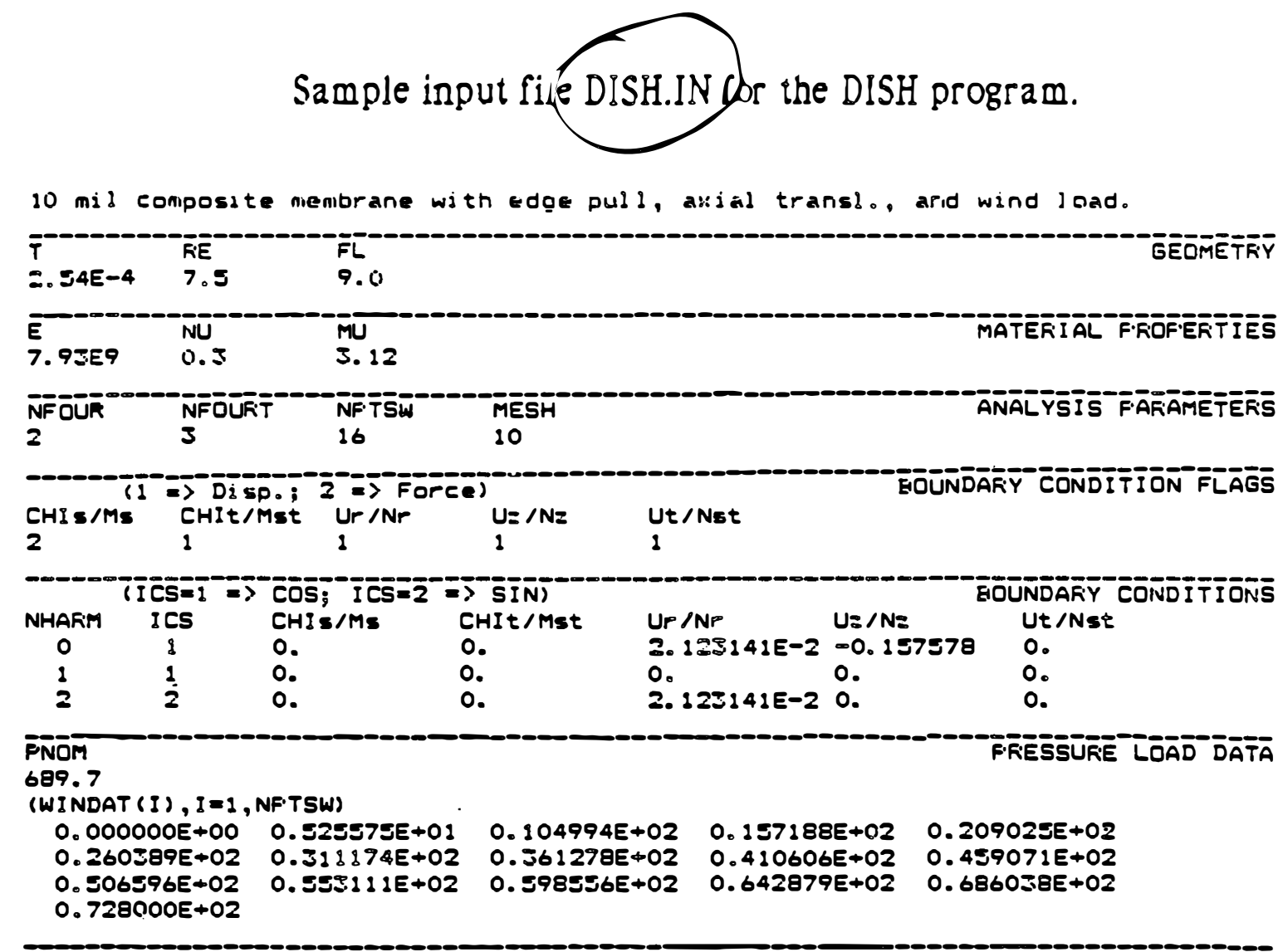

Sample input file DFCOMP.IN for the post-processor DFCOMP.

Input File DFCRMF. IN, for the pestprocessor DFCOMF.

$\begin{array}{lll}\text { ITER } & \text { NFTS } & \text { RANGE } r, \text { theta (d) } \\ \text {.FALSE. } 4,2 & \text { t. O,7.J } 0 ., 96 .\end{array}$



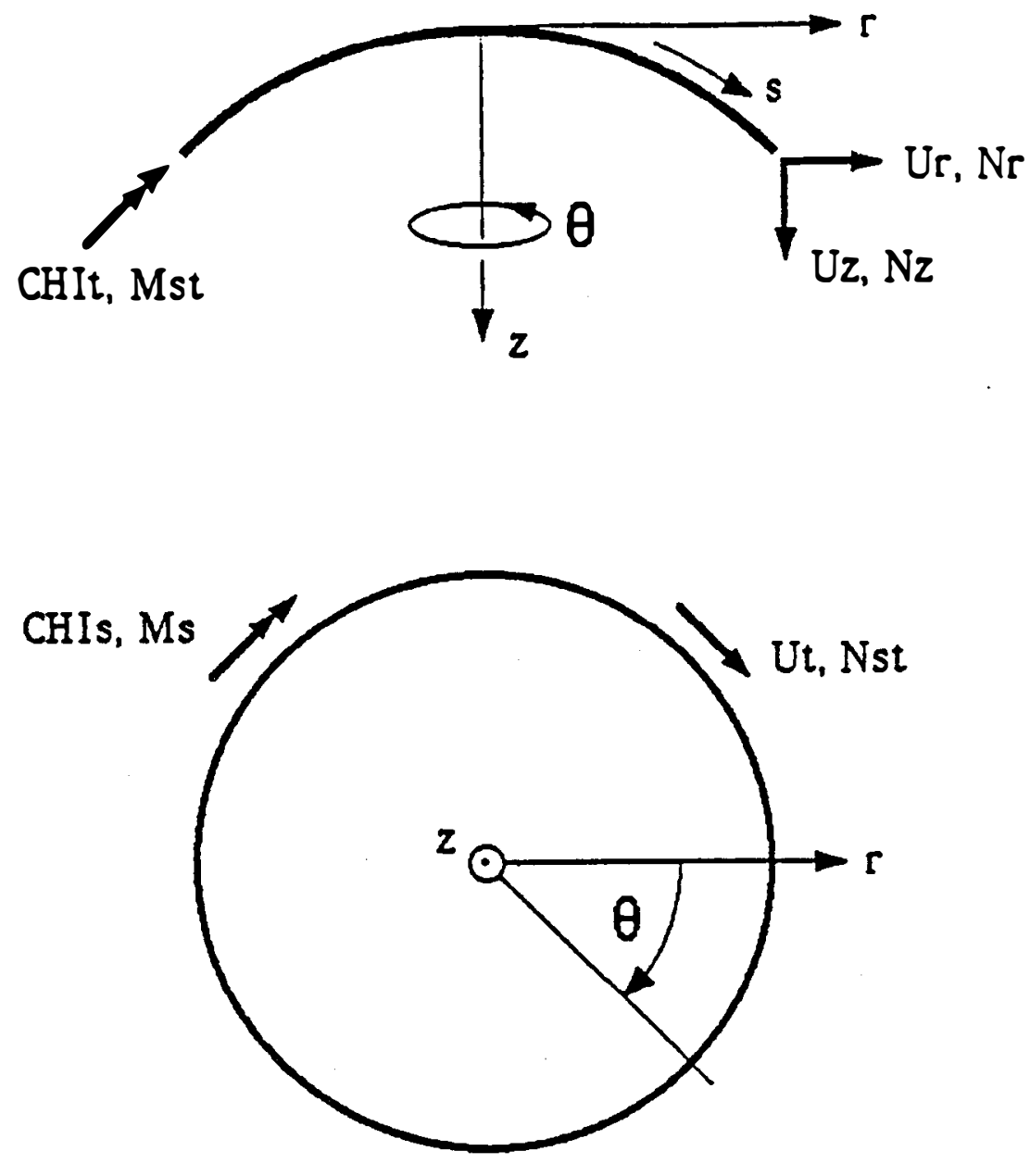

Sign conventions for forces, displacements, moments, and rotations at edge of dish. 


\section{Sample output file DFCOMP.OUT for the post-processor DFCOMP.}

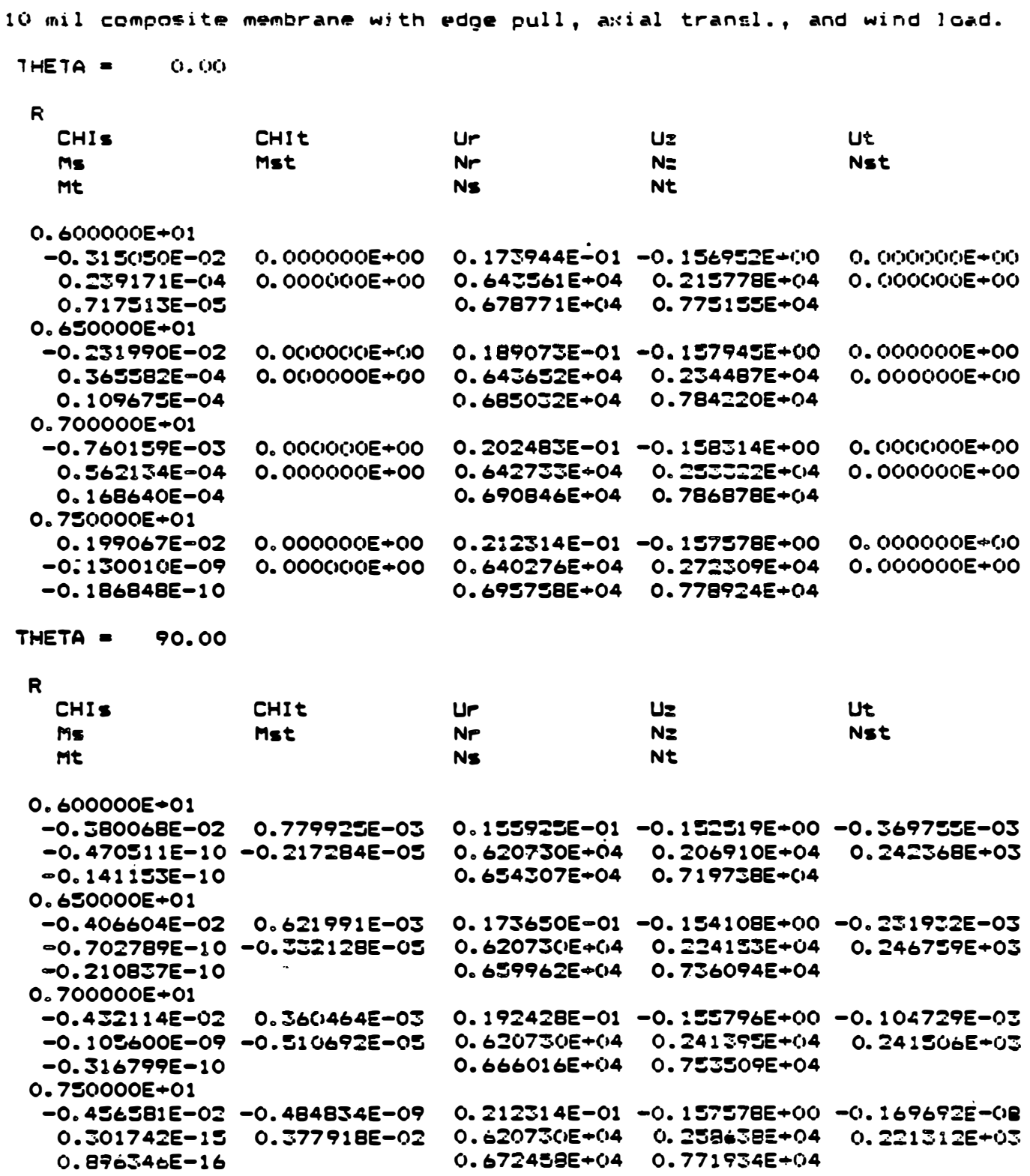




\section{Output from the postprocessor DINFO.}

10 mil coninozite miemorane wish edoe pull, ai:ini trangl., arid wind loas.

memorane Solution bata:

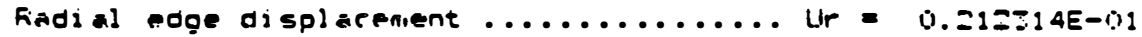

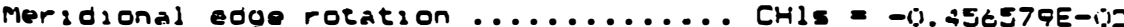

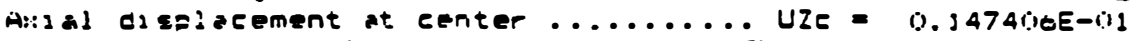

Aprnronimate toeal length ............ FLriew $=0.014284 E+01$

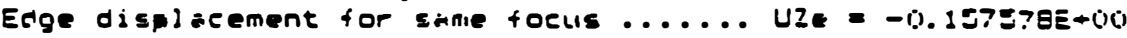

Edge Effect Data:

Short decay distance .............. DELTAS $=0.417920$ E-0.2

Long decay distance .............. DELTAL = 0.555907E-01

Pressurizasion parameter ............ RHO = 0.4ZS467E+nS

Decay distince for =ero pressure ..... DELTA = 0.172000EE+Ci0

\section{Nodal displacements and rotations from a SUPERSAP analysis.}

- Brove

$-4.15: 5 E-0.04$

$-5.9511 E-i 14$

$-4.6802 E-1.14$

$-2.0=2.5 E-1.14$

$-1.25 .5 \div E-1.12$

$-5.587 T E-03$

$-8.81 \div 1 E-0.03$

$-5.597: E-0.3$

-2. $2590 \mathrm{j}-03$

-1. 2870E-0J

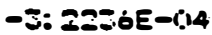

ล.7ЕISE-04

כ. $59=0 E-(14$

S. S985E-

ב. $.894 E-014$

. OSOUE-OO
2.842ZE-0.5 - 2.6178E-02 -1.90.8EE-02

$2.4624 E-105-2.815 \Xi E-02-1.275 E E-02$

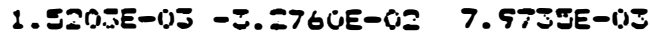

इ. $5=76 E-0.04-3.5 .550 .0-02 \quad=.6797 E-02$

-6.5044E-014 -2. 525EE-U2 4. Z752E-02

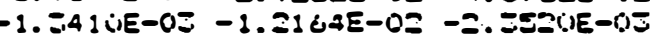

1. 6(14dEE-07 -1.4145E-07 -6. 60168E-02

1. $95 \triangle 4 E-03-2.2027 E-i J 2-=$. (IBBZE-UZ

1.9951E-0J - $.0 .10 E-02-2.2748 E-02$

2. $62=3 E-0 I-6.7805 E-02-2.1650 E-02$

ב.46กBE-UI -7.5BS6E-0I - 1. J08OEE-02

4. I780E-0I -8.0142E-0I -4.59 S0E-03

S. =-56E-03 -8.4190E-02 - $2.490 .5 E-03$

6. इЕ1JE-OI -8.8444E-0I -4.7290E-OI -

6.98ZSE-03 -9.2099E-02 -7.0824E-0J -

7. $4746 E-03-9.4462 E-02-7.9 B 01 E-0 J$

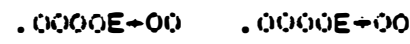

8. $025 E E-6.04-6.1526 E-i .14$

$7.2640 E-105-1.0021 E-0.0$

$2.5576 E-02-9.9551 E-0.4$

2.7410E-0. $-1.015 S E E-0.5$

- E.0181E-C.: 1.0545E-

$-1.4670 .0-1.1$ 6.280.7E- 0

$-2.6176 E-02$ 1. 2EEZE-6I

$4.7994 E-02-4.2494 E-65$

4. 3570E-02 - 1. 92EZEE- 05

1.5t5OE-0. $-1.465 .9 E-()=$.

$-5.8971 E-1.14-1.1=21 E-0.0$

- - 3649E-0.3 - $9.6617 E-(.14$

-1.5782E-0I -7.7579E- .14

- $.0644 E-0.14-5.5=321 E-1.14$

1. 7080E-05 - $5.68595-0.14$

$.0000 E+00$ : OSOOCIE - OO 


\section{Input file FSCOEF.IN to the Fourier analysis program FSCOEF.}

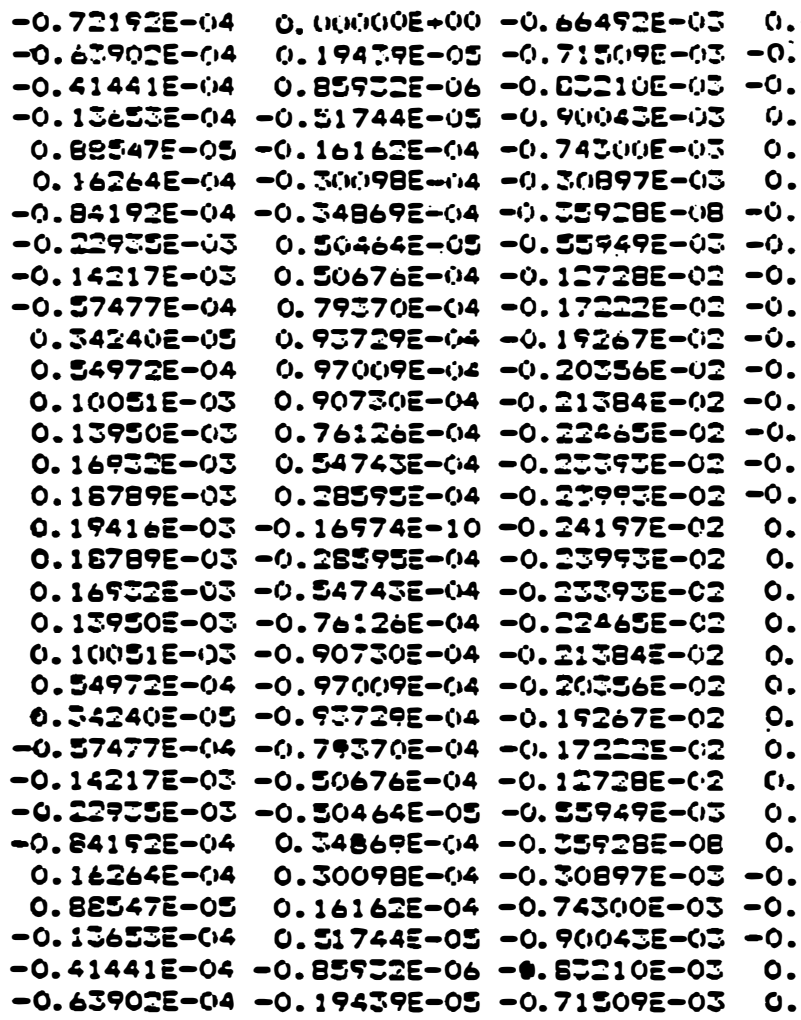

0. 8SSC17E-Ci4 - $0.28065 E-C=$ $-0.258 E 2 E-(1)-0.46079 E-03$

$-0.20168 E-c 13$ 0.87E2=E-0E $-0.291 E=E-04 \quad 0.72290 E-03$ $0.65864 E-C 14$ 0.17709E-DJ $0.57168 E-(14-0.21561 E-03$

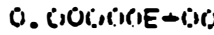
$-0.16,75.5 E-(.44$ $-0.2 \varepsilon=57 E-(.14$ $-0.1744 E E-04$ $-0.189517-0.14$ 0. $190==E-0.14$ $0.10 .196=E-10=$ 0. $=2082 \equiv-0.14$ -0.75(0.) $-0 .= \pm 62$ PE- 1.14

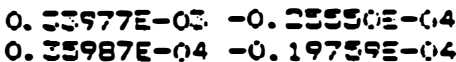
$-0.1075 .4 E-(14 \quad-0.1$ ö86.JE 0.11216E-0 $0.0 .9506-5-05$

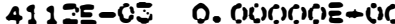
0.11216E-1JS 0. $93016=E-05$ $0.1=5$ I. $5 E-(14$ $0.1636 . \bar{E}-(14$ $0.15759 E-0.14$ 0. IEะE(1E-64 $0.505: 9 E-0.14$ $0.750 .9 \div \equiv-0.14$ -0. =-OE=E- :4 $-C .205 \dot{e}=E-6: \overline{3}$

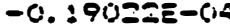
$0.1 E 95: E-0.14$ O. $17445 E-(14$ $0.185=-E E-04$ $0.107=8 E-(14$ 


\section{$\underline{\text { Prescribed Radial Edge Deformation: } u_{r}=U_{0} \cos 2 \theta}$}
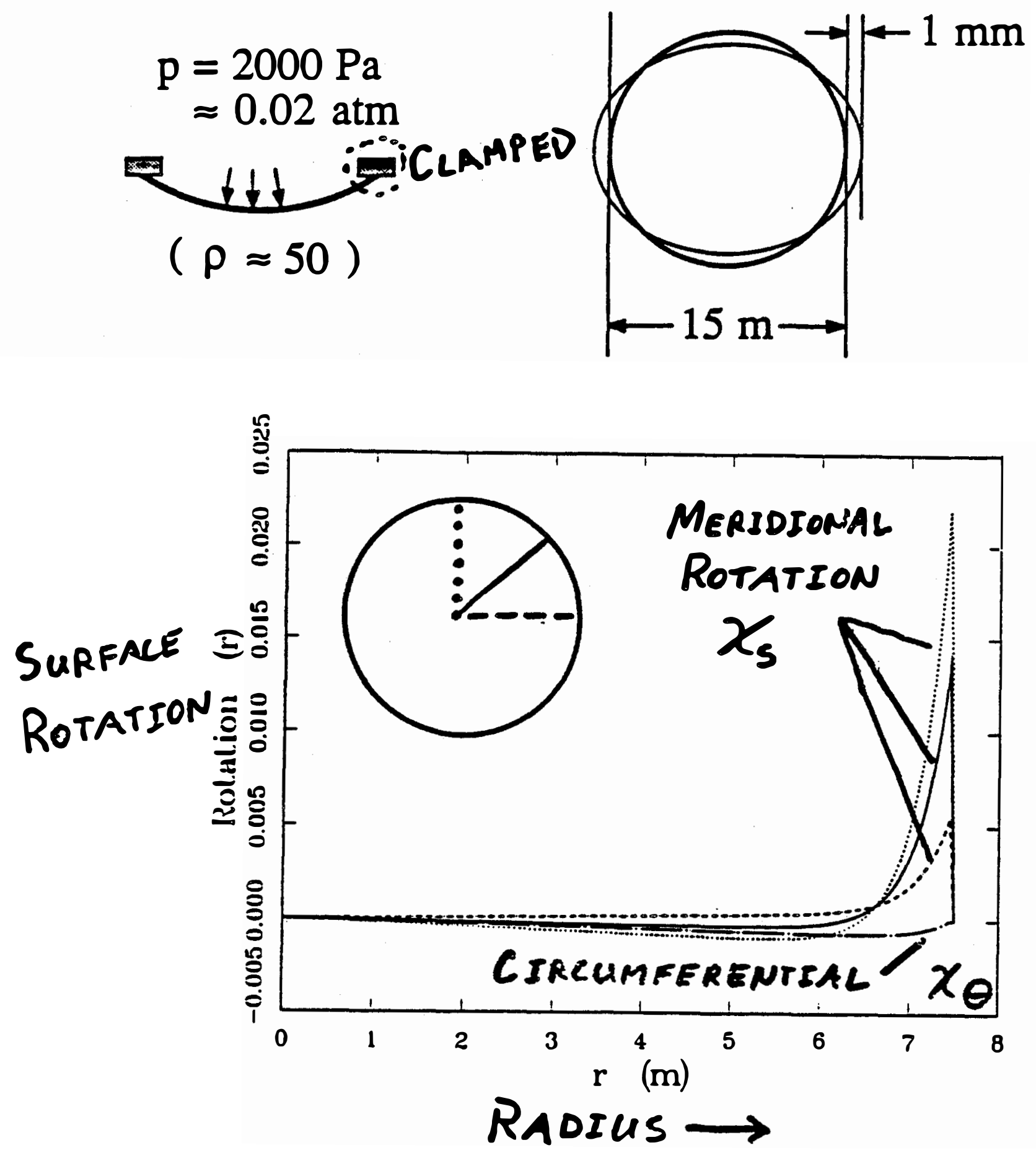
Prescribed Verteche EDge

DEFORMATION: $U_{z}=U_{0} \cos 2 \theta$

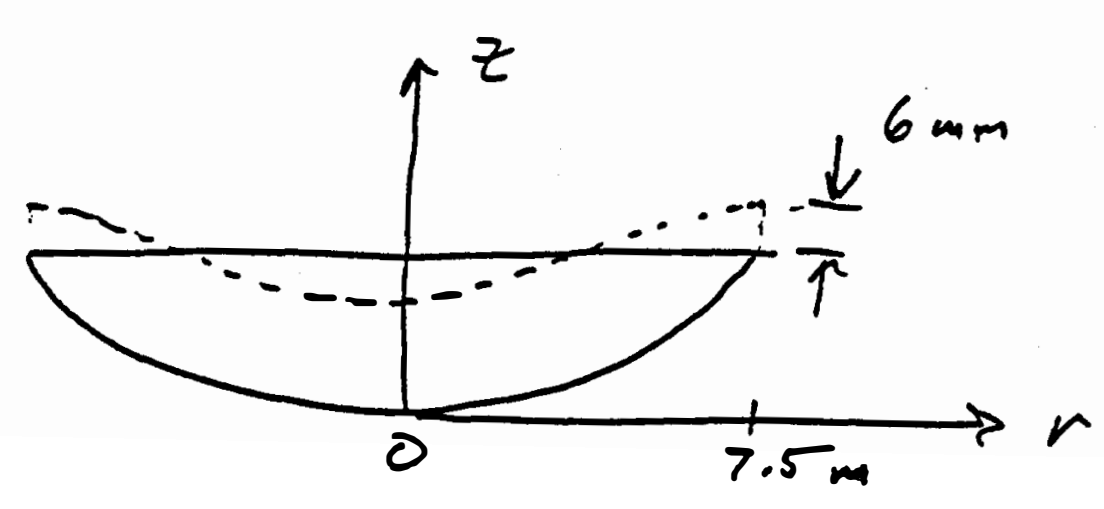

Stab. Pressure $=1000 \mathrm{~Pa}$

$20 \mathrm{mil}$ Composite Membrane

$$
(E=7.93 \mathrm{GPa})
$$

C -17 
ANSYS F.E. MODEZ (TUAN)

21 nodes radial

24 nodes circumferential

Triangular Shall elemant
(6 d.o.t. per node)

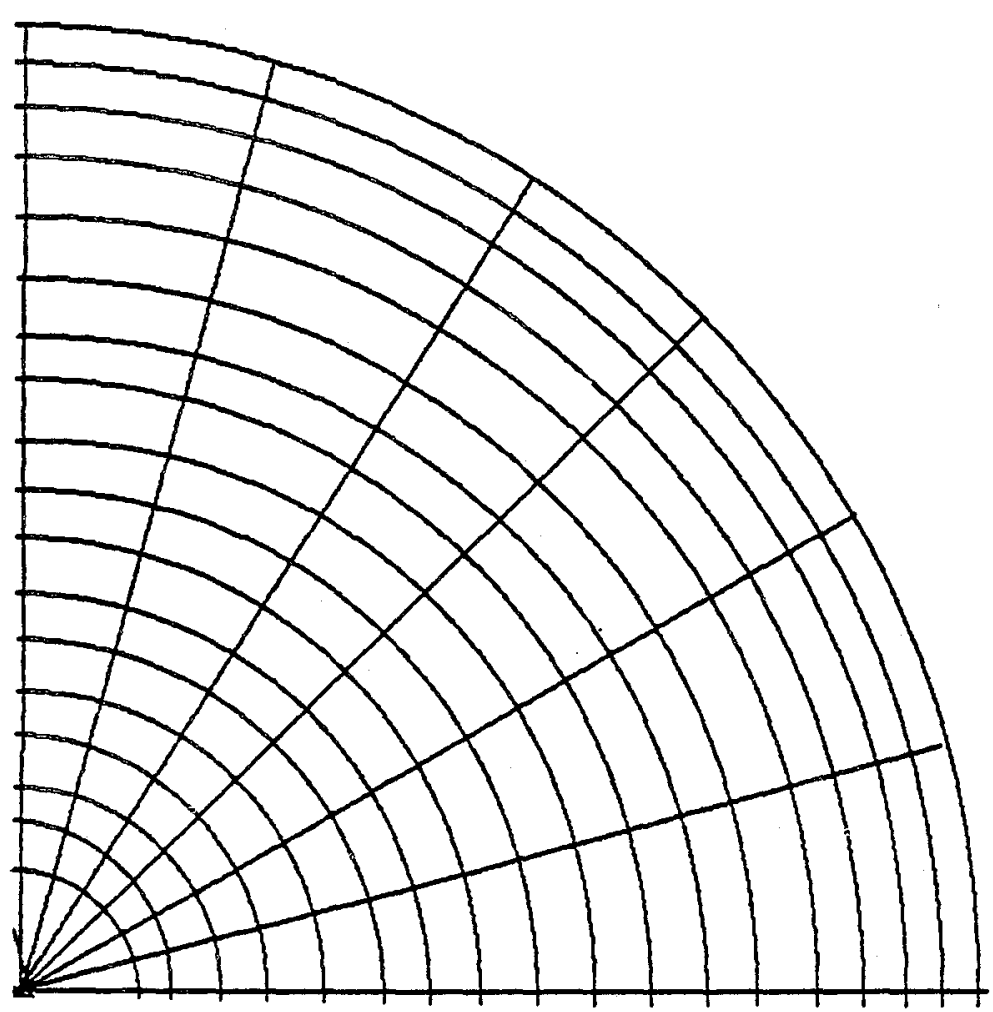

(Artest's Concepteon)

$\mathrm{C}-18$ 


\section{RADIAL DISPLACEMENT}

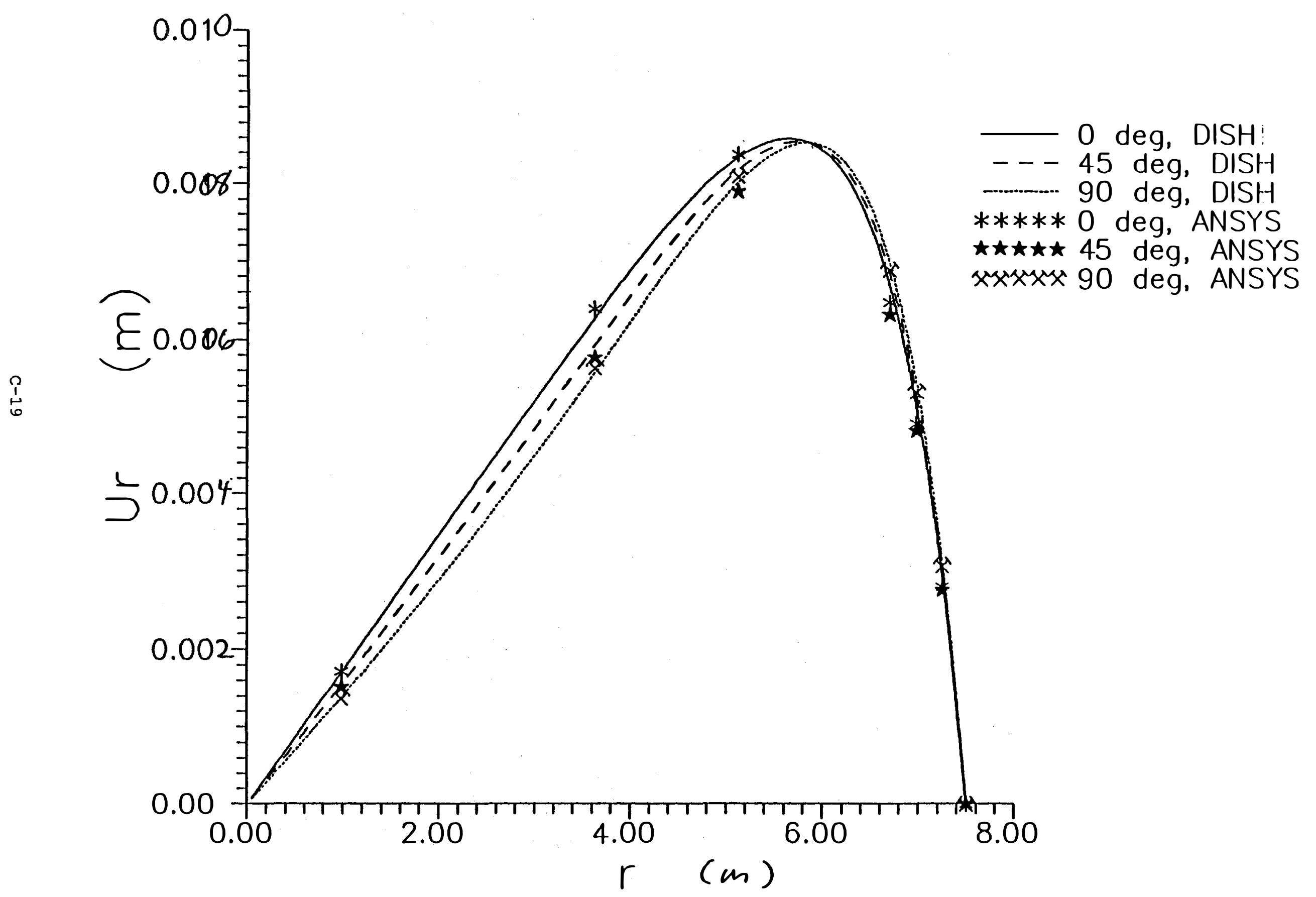




\section{CERCUMFERENTEAL DESPLACEMENT}

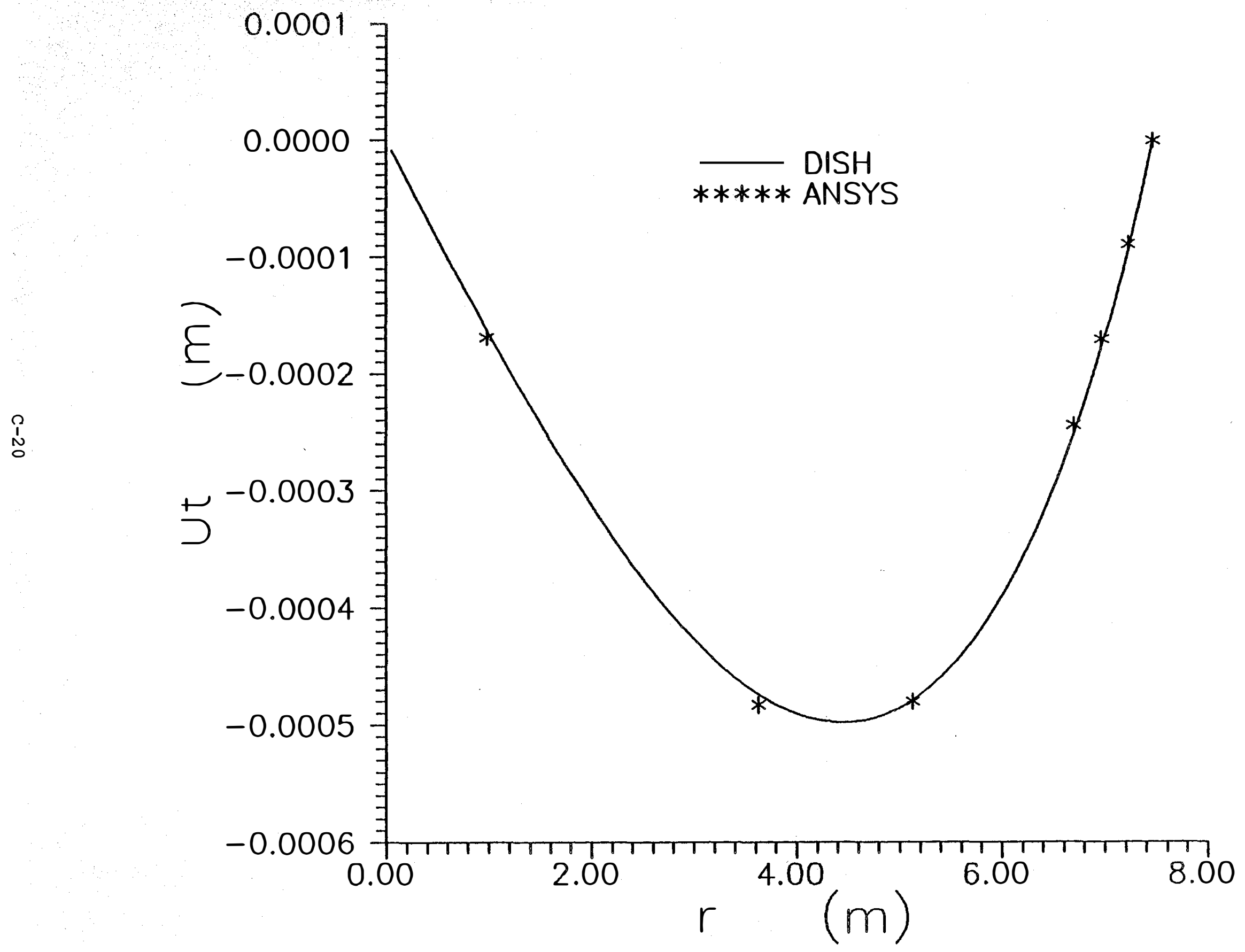


VERTICAL IJISPLACEMENT

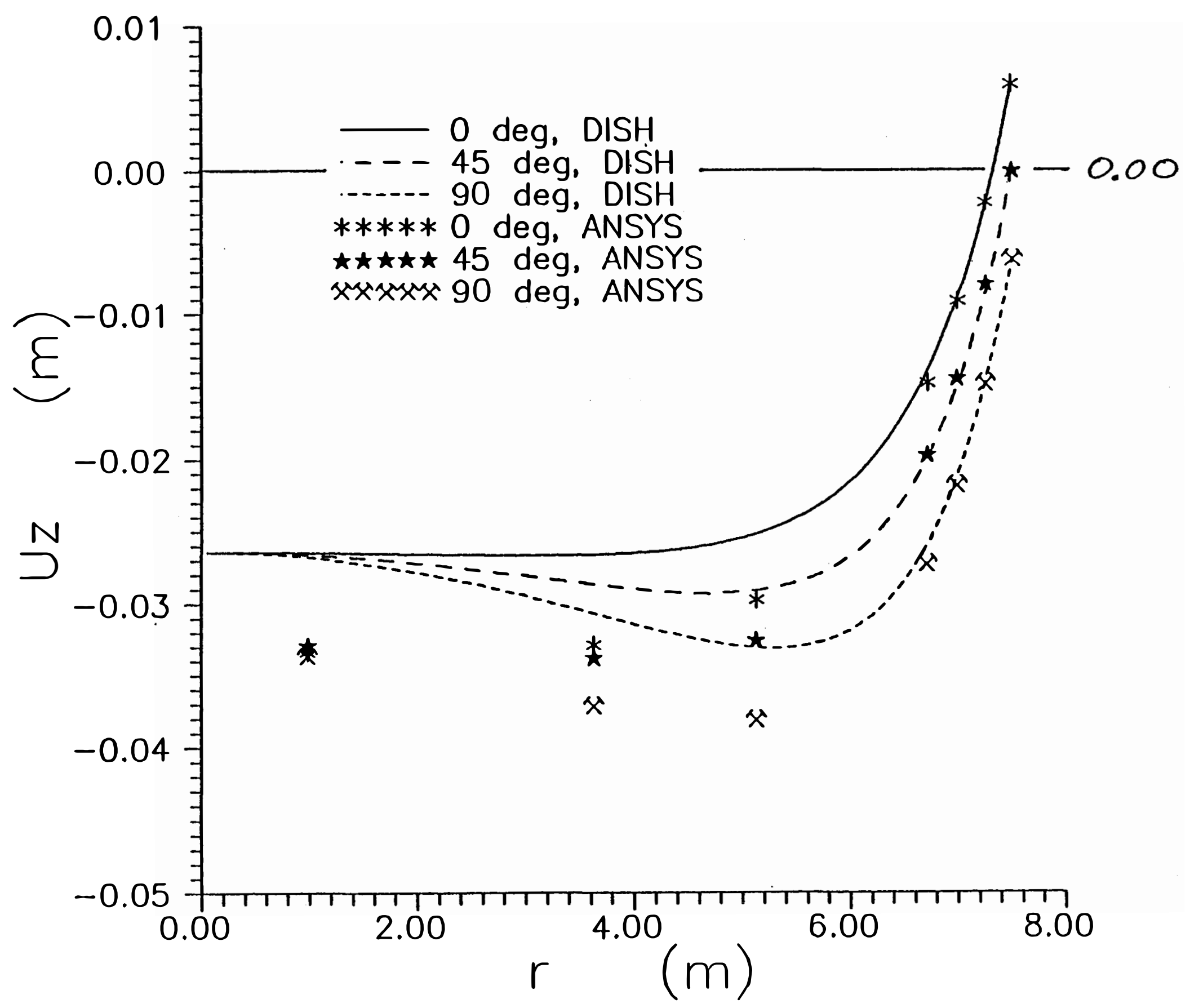




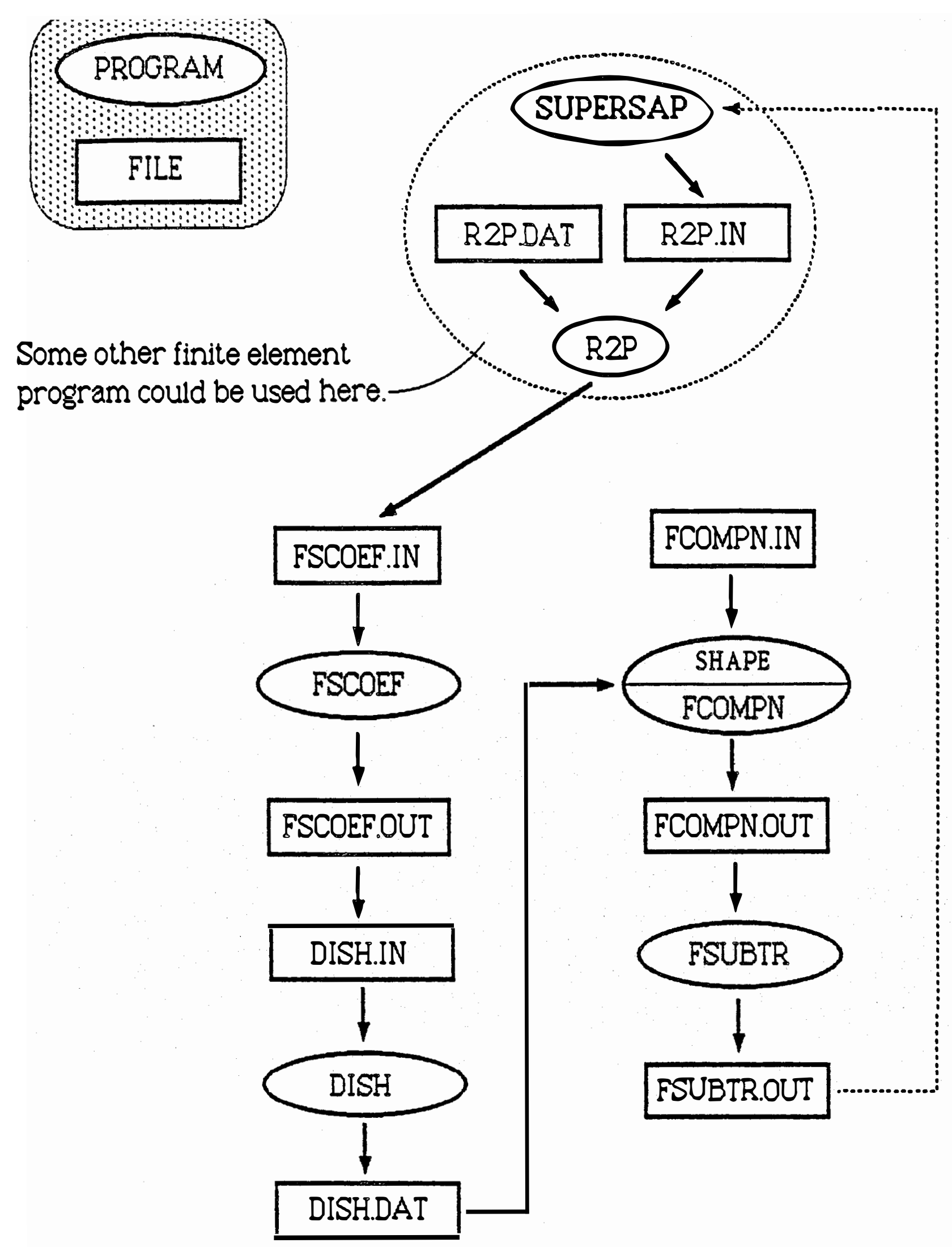

Data path flowchart for enforcing finite element nodal ring displacements in the dish structural analysis. 


\section{Load Case for DAN-KA Run ASDF4E}

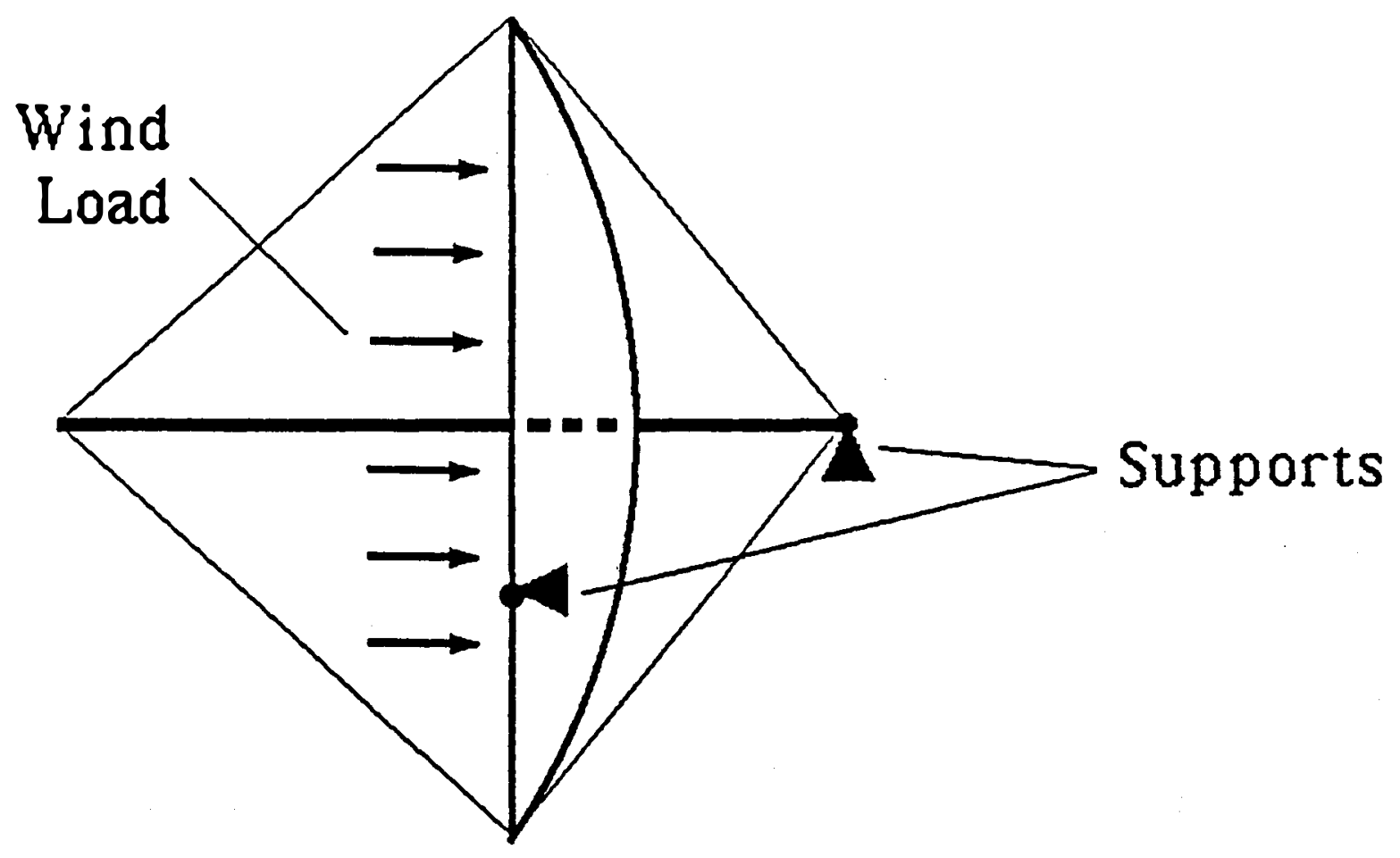


Axial Displacement Uz from DAN-KA Run ASDF4E

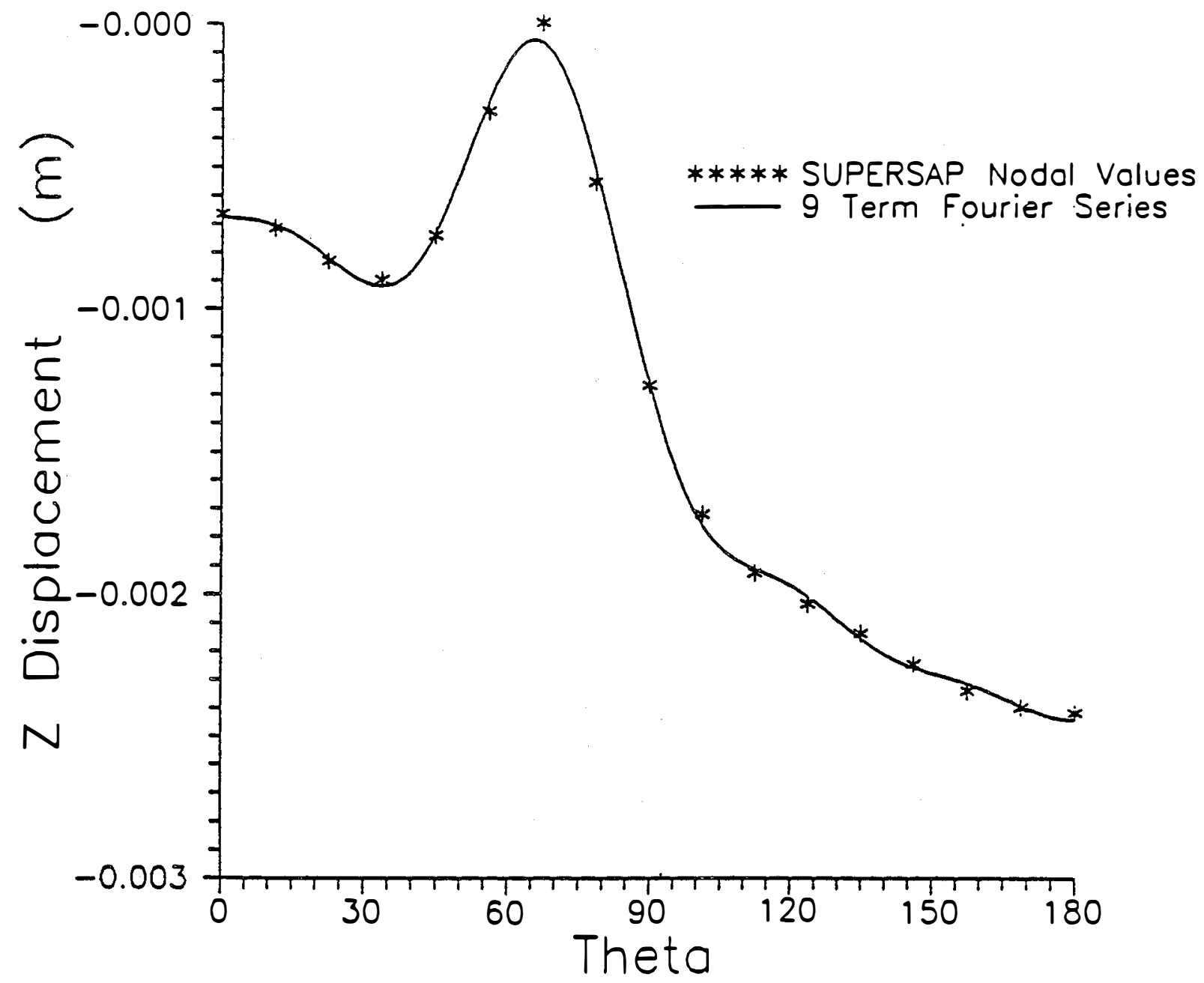

Comparison of SUPERSAP nodal displacements liz with the nine term Fourier series representation. 


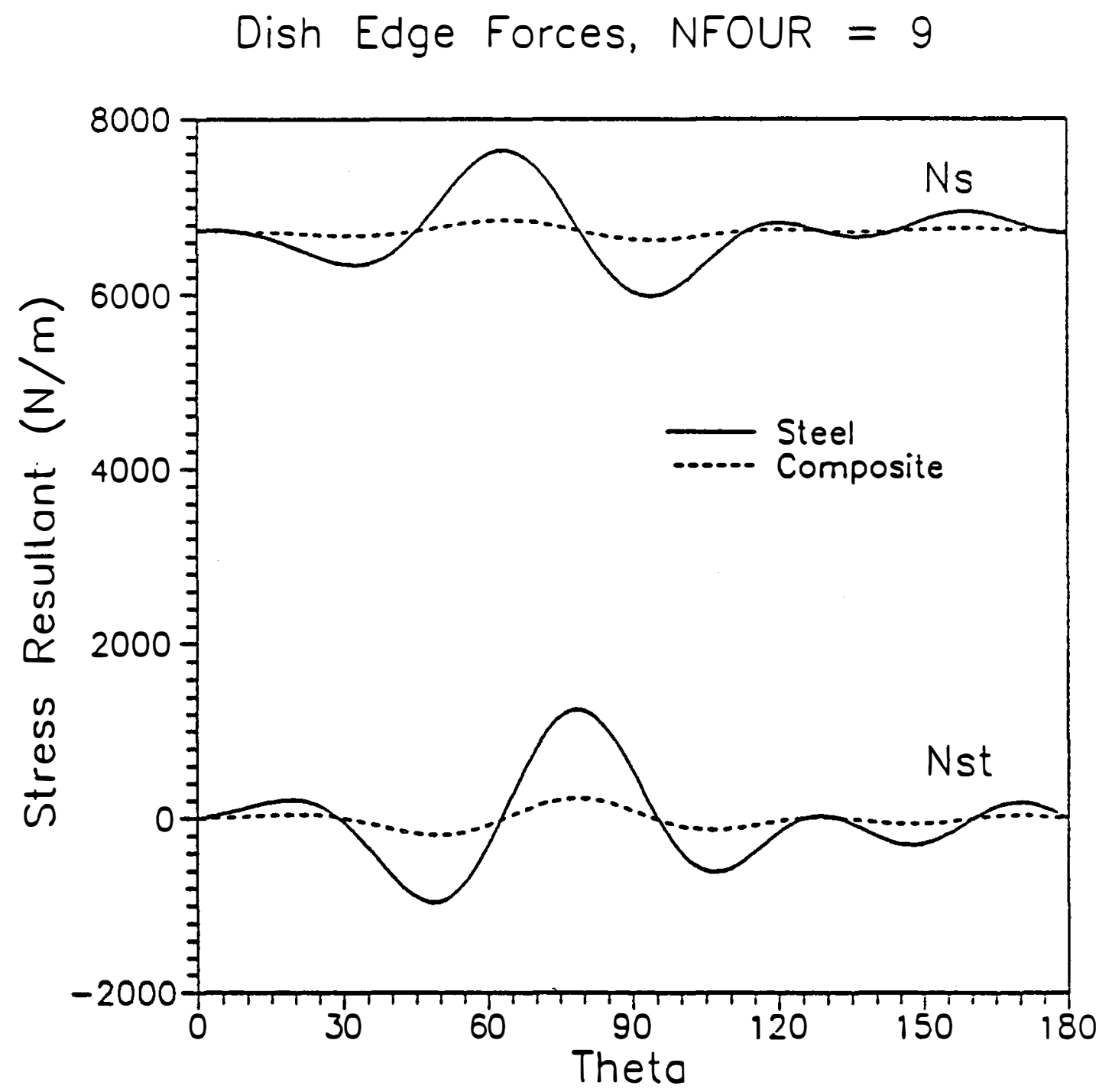

Meridional and tangential stress resultants Ns and Nst due to ring displacements. 
Equilibrium of Membrane and Support Structure
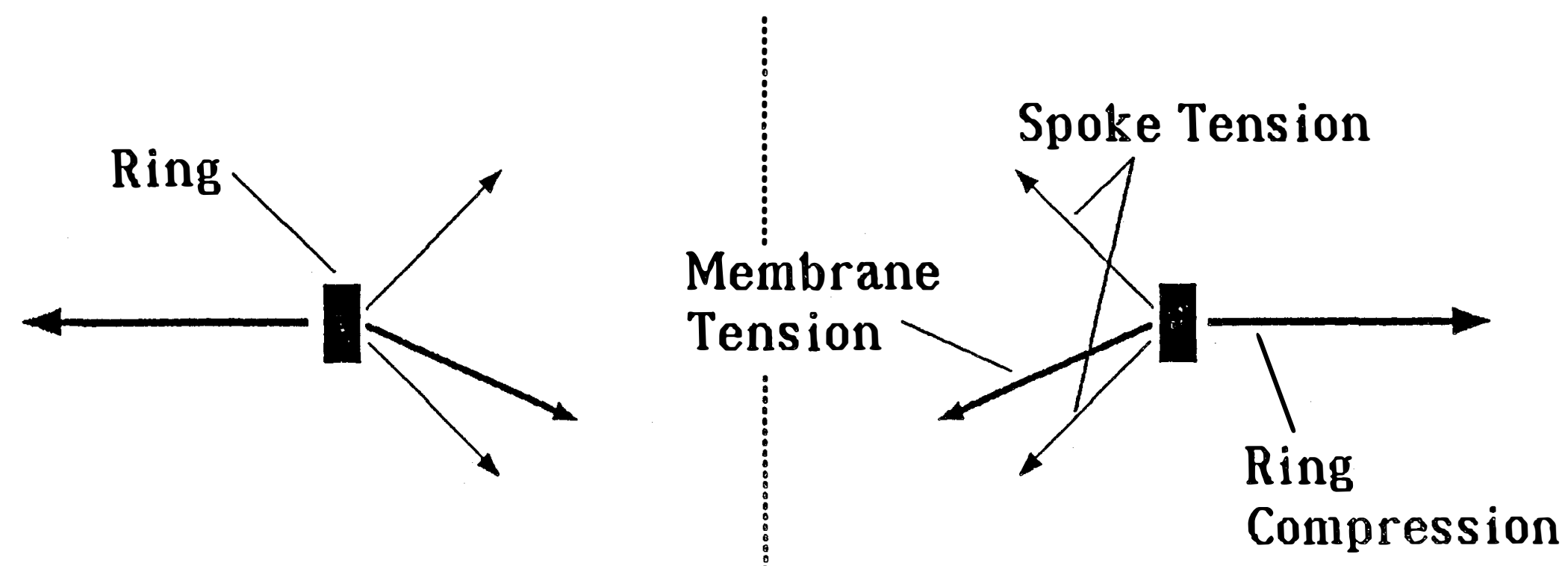
10 nil composite Menhrane; ASDFAE eilge displacements edge pull, axial transl. Dish Radius= 1.56; Focal Lenyth= 9.00; Dish Surface Location

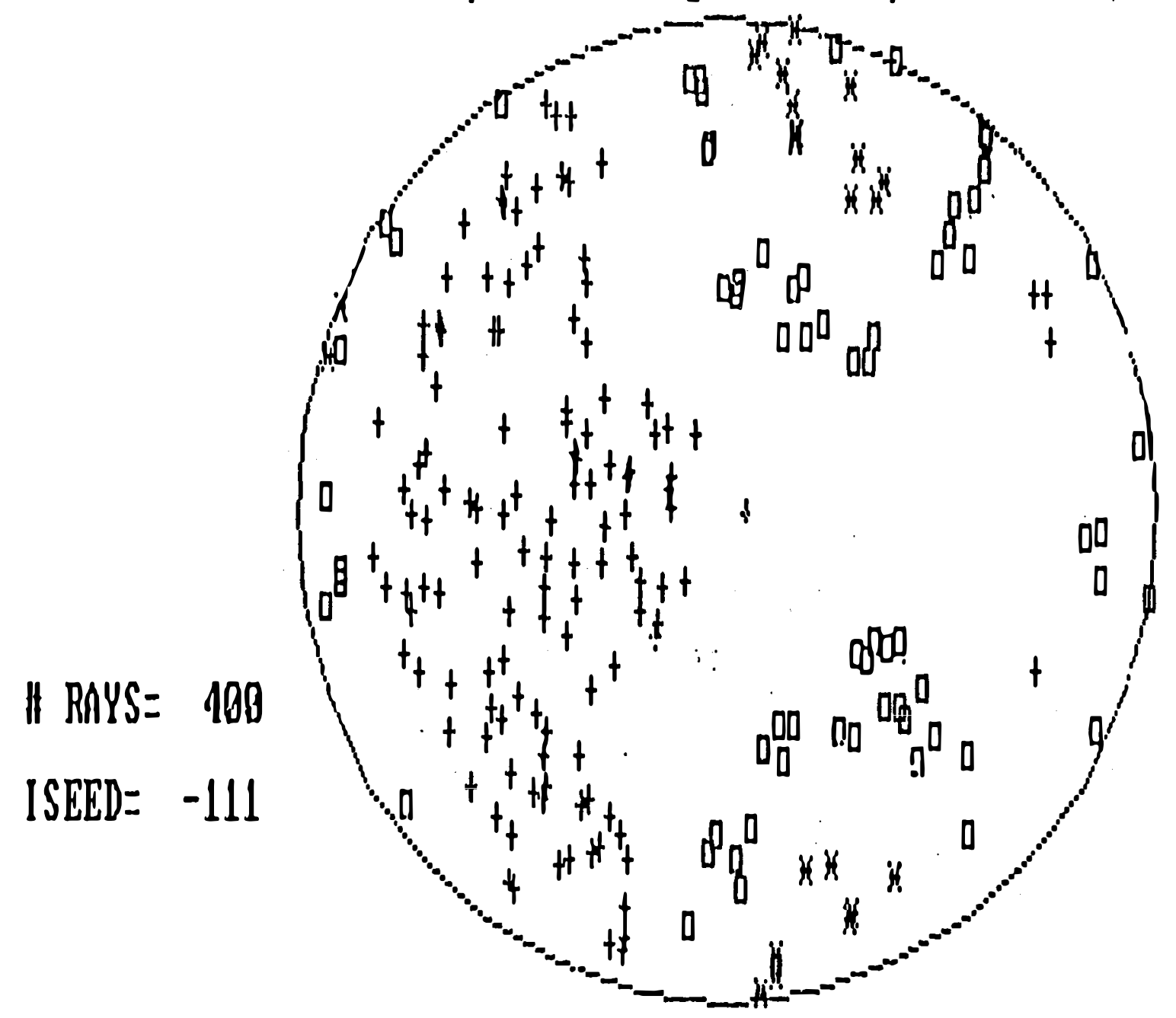

$N R=0$

$N T=0$ 